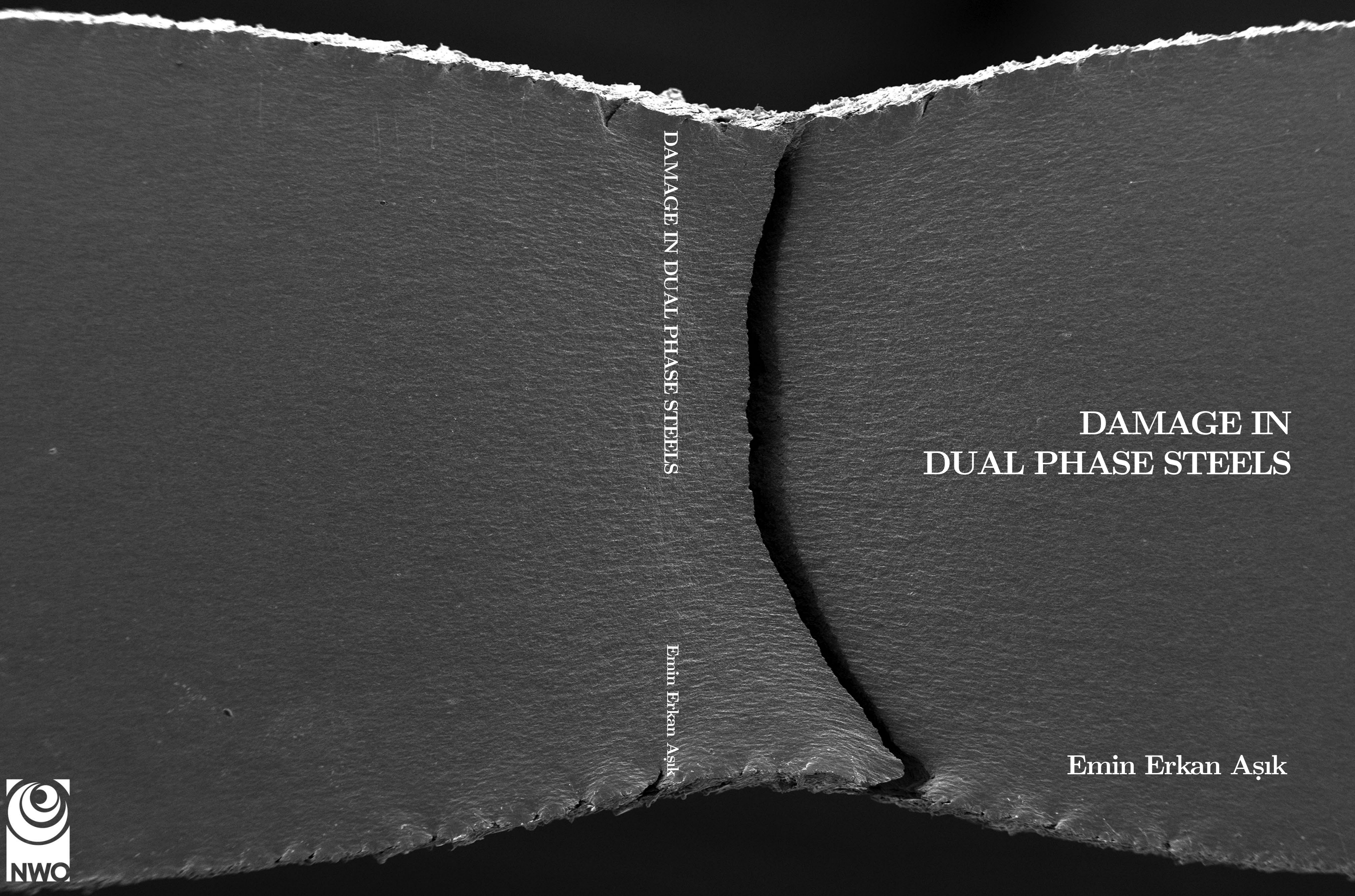


This page intentionally left blank. 


\section{DAMAGE IN DUAL PHASE STEELS}

Emin Erkan Aşık 
This dissertation has been approved by:

supervisor

prof. dr. ir. A.H. van den Boogaard

co-supervisor

dr. ir. E.S. Perdahcıoğlu

Cover design: Sıla Akman \& Erkan Aşık

Printed by: Gildeprint, The Netherlands

Lay-out: Erkan Aşık

ISBN: 978-90-365-4881-6

DOI: $10.3990 / 1.9789036548816$

This research was carried out under project number F61.1.13486 in the framework of the Partnership Program of the Materials innovation institute M2i (www.m2i.nl) and the Foundation of Fundamental Research on Matter (FOM) (www.fom.nl), which is part of the Netherlands Organization for Scientific Research (www.nwo.nl).

(c) 2019 by E. E. Aşık, Enschede, The Netherlands. All rights reserved. No parts of this thesis may be reproduced, stored in a retrieval system or transmitted in any form or by any means without permission of the author. Alle rechten voorbehouden. Niets uit deze uitgave mag worden vermenigvuldigd, in enige vorm of op enige wijze, zonder voorafgaande schriftelijke toestemming van de auteur. 


\section{DAMAGE IN DUAL PHASE STEELS}

\section{DISSERTATION}

to obtain

the degree of doctor at the Universiteit Twente, on the authority of the rector magnificus,

Prof.dr. T.T.M. Palstra, on account of the decision of the graduation committee to be publicly defended

on Wednesday 13 November 2019 at 14.45

by

Emin Erkan Aşık

born on the 20th of September 1985

in Ankara, Turkey 


\section{Graduation Committee:}

Chairman / secretary prof. dr. G.P.M.R. Dewulf

Supervisor

Co-supervisor

Committee Members prof. dr. ir. A.H. van den Boogaard

dr. ir. E.S. Perdahcıoğlu

dr. ir. T.C. Bor

prof. S. Leen

prof. dr. ir. J. Sietsma

prof. dr. ir. C.H. Venner 


\section{Summary}

Advanced High Strength Steel (AHSS) sheets play an increasingly crucial role in engineering applications such as structural parts in the automotive industry, due to their desired combination of strength and ductility. Dual phase (DP) steels stand out among other AHSS grades since their mechanical properties can easily be adjusted by conventional heat treatment procedures without addition of expensive alloying elements. Versatile mechanical properties of DP steels originate from the presence of two mechanically diverse phases - ferrite and martensite - and the way they are dispersed throughout the microstructure. This dual phase structure with complex morphology brings scientific challenges for understanding and predicting the mechanical response especially in the damage regime.

Sheet metals are usually subjected to forming operations which involve deformation of the sheet into a desired shape. During forming, the sheet undergoes a large variety of stress states while being plastically deformed. In the case of DP steels, the formability of the sheet is strongly influenced due to development of ductile damage, which leads to loss of load carrying capacity or even unpredicted failure. Although damage evolution in single phase materials is well understood, the observations on more complex multi-phase alloys, such as DP steels, often exhibit contradicting outcomes. This is due mainly to the large diversity of the microstructural features even for the same grade of material and the resulting variations in the heterogeneous distribution of stress and strain fields. In order to develop an in-depth understanding of damage initiation and evolution, it is therefore crucial to understand the effect of microstructural morphology and interaction between the phases.

In this research, the effect of microstructural features on damage behavior is studied with a special emphasis on evolution of plastic deformation heterogeneity in microscale. This is carried out by performing experimental and numerical work on a ferritic-martensitic DP600 steel sheet from the AHSS family.

The experimental investigations are focused on two aspects: (i) evolution of plastic deformation heterogeneity and lattice orientations in the grain scale and (ii) active damage mechanisms and distribution and evolution of damage during deformation. The former is carried out by electron backscatter diffraction (EBSD) measurements. The investigations revealed that before the deformation small orientation variations are present and hence there are geometrically necessary dislocations (GND) at the ferrite grain boundaries. With increasing deformation, lattice orientation spread of 
the grains increases due to heterogeneity in plastic deformation and therefore the GND content also increases. The density of GNDs tend to be higher around grain boundaries, but they also form highly non-uniform distributions inside the ferrite grains. Moreover, investigations around the voids showed high GND content mostly on one side of the voids. For the latter aspect metallographic cross sections are investigated and image analysis are performed. Two main mechanisms are identified for void formation: (a) by cracking of martensite and (b) decohesion at the ferrite grain boundaries. Voids formed by cracking of martensite grow perpendicular to the loading direction after complete failure of the martensite island. Therefore, these voids have a thickness of the martensite island from which they formed within. The voids that are formed at the boundaries stay thin and mostly grow along the loading direction, causing a higher length to thickness ratio. Image analysis of the spatial distribution of the voids revealed a non-uniform distribution along the thickness of the sheet which is correlated with the distribution of martensite bands.

Accordingly, in order to capture the effect of lattice orientations and plastic strain heterogeneity, a strain gradient enhanced rate-independent crystal plasticity framework is implemented as a user subroutine for the commercial finite element package ABAQUS/STANDARD. In this generic framework, a lower order approach is employed for the gradient enhancement. It was shown that the model is capable of predicting both structural and microstructural size effects. The model also showed that the local stress and strain states deviated from the macroscopically prescribed conditions on the RVE.

Numerical investigations of DP microstructure are focused on the effect of martensite distribution on (i) the stress and strain partitioning, (ii) the response of damage indicators and (iii) the evolution of pre-existing damage incidents. Firstly, it was shown that the average stress and strain of ferrite and martensite are affected significantly by the distribution and content of the phases. In particular, the martensite percentage affects the stress and strain partitioning due to the hardness contrast as well as the induced strain gradients within ferrite. On the other hand, the effect of martensite distribution is more pronounced on the initial hardening rate of the RVE. Secondly, after comparison of locations of experimentally observed damage incidents and numerical ones, it was seen that a plastic work based damage indicator is suitable for predicting damage locations and damage susceptibility of the microstructure. It was found that an increase in martensite content enhances the possibility of damage in both ferrite and martensite and a banded morphology triggers more damage in the martensite phase. Finally, it was found that evolution characteristics of voids are highly dependent on their locations and surroundings in other words the local stress and strain state. Moreover, the evolution does not follow a monotonic trend. Interestingly, in the simulations during tensile stretch some preexisting voids shrink, which is an indicator of locally compression or shear type of loading. This also shows the variation of local state from the average prescribed condition. In general, a higher void growth rate is observed for the voids located in between individual martensite islands for the other voids. 


\section{Samenvatting}

Platen van (geavanceerde) hogesterkte staalsoorten (AHSS) spelen een steeds belangrijkere rol in technische toepassingen zoals dragende onderdelen in de automobiel-industrie, vanwege hun gewenste combinatie van sterkte en vervormbaarheid. Twee fasen (DP) staal onderscheidt zich van andere AHSS-kwaliteiten omdat de mechanische eigenschappen gemakkelijk kunnen worden aangepast door conventionele warmtebehandelingsprocedures zonder toevoeging van dure legeringselementen. De veelzijdige mechanische eigenschappen van DP-staal komen voort uit de aanwezigheid van twee mechanisch diverse fasen - ferriet en martensiet - en de manier waarop ze door de microstructuur worden verspreid. Deze tweefasenstructuur met complexe morfologie brengt wetenschappelijke uitdagingen met zich mee voor het begrijpen en voorspellen van de mechanische respons, met name in het schadegebied.

Plaatmetalen worden gewoonlijk onderworpen aan omvormbewerkingen die vervorming van de plaat tot een gewenste vorm met zich meebrengen. Tijdens het omvormen ondergaat de plaat een grote verscheidenheid aan spanningstoestanden en plastische vervorming. De vervormbaarheid van de plaat is beperkt door de ontwikkeling van ductiele schade, wat leidt tot verlies van draagvermogen of zelfs tot onvoorspelbaar faalgedrag. Hoewel de ontwikkeling van schade in materialen met een enkele fase goed wordt begrepen, vertonen de waarnemingen aan complexere legeringen met meerdere fasen, zoals DP-staal, vaak tegenstrijdige resultaten. Dit is voornamelijk te wijten aan de grote diversiteit van de microstructurele kenmerken, zelfs voor dezelfde materiaalsoort en de resulterende variaties in de heterogene distributie van spannings- en rekvelden. Om een diepgaand begrip van schade-initiatie en -evolutie te ontwikkelen, is het daarom cruciaal om het effect van microstructurele morfologie en interactie tussen de fasen te begrijpen.

In dit onderzoek wordt het effect van microstructurele kenmerken op het gedrag van schade bestudeerd met een speciale nadruk op de ontwikkeling van heterogeniteit van plastische vervorming op microschaal. Dit wordt uitgevoerd door experimenteel en numeriek werk uit te voeren op een ferritisch-martensitische DP600-staalplaat uit de AHSS-familie.

Het experimenteel onderzoek is gericht op twee aspecten (i) ontwikkeling van heterogeniteit van plastische vervorming en roosteroriëntaties op korrelschaal en (ii) actieve schade-mechanismen en distributie en evolutie van schade tijdens vervorming. Het eerste aspect wordt uitgevoerd door elektronen-backscatter diffractie (EBSD) metingen. Uit dit onderzoek blijkt dat er vóór de vervorming kleine 
oriëntatievariaties zijn en dus geometrisch noodzakelijke dislocaties (GND) aan de grenzen van de ferrietkorrels. Met toenemende vervorming neemt de spreiding van de roosteroriëntatie van de korrels toe als gevolg van heterogeniteit in plastische vervorming en daarom neemt ook het GND-gehalte toe. De dichtheid van GND's is meestal hoger rond korrelgrenzen, maar ze vormen ook een zeer niet-uniforme verdeling binnen de ferrietkorrels. Bovendien toonde onderzoek rond de holtes een hoog GND-gehalte aan, meestal aan één kant van de holtes. Voor het laatste aspect worden metallografische dwarsdoorsneden onderzocht en beeldanalyses uitgevoerd. Twee hoofdmechanismen worden geïdentificeerd voor de vorming van holtes (a) door breken van martensiet en (b) loslating aan de grenzen van de ferrietkorrels. Holtes gevormd door breken van martensiet groeien loodrecht op de belastingsrichting na volledig falen van het martensieteiland. Daarom hebben deze holtes dezelfde dikte als het martensitische eiland waaruit ze zijn ontstaan. De holtes die aan de randen worden gevormd, blijven dun en groeien meestal in dezelfde richting als de belasting met een hogere lengte-dikteverhouding. Beeldanalyse van de ruimtelijke distributie van de holtes onthulde een niet-uniforme distributie langs de dikte van de plaat die gecorreleerd is met de distributie van martensietbanden.

Om het effect van roosteroriëntaties en plastische rekheterogeniteit mee te kunnen nemen, is een met rekgradiënt uitgebreid reksnelheidsonafhankelijk kristalplasticiteitsmodel geïmplementeerd als een gebruikerssubroutine voor het commerciële eindige elementenpakket ABAQUS/STANDARD. In dit generiek kader wordt een benadering van lagere orde gebruikt voor de gradiëntverbetering. Er werd aangetoond dat het model in staat is om zowel structurele als microstructurele grootte-effecten te voorspellen. Het model toonde ook aan dat de lokale spanningsen rekcondities verschillen van de macroscopisch voorgeschreven condities op de RVE. Numerieke onderzoeken naar DP-microstructuur zijn gericht op het effect van martensietdistributie (i) op de distributie van spanning en rek, (ii) reactie van schade-indicatoren en (iii) evolutie van reeds bestaande schade-incidenten. Ten eerste werd aangetoond dat de gemiddelde spanning en rek van ferriet en martensiet aanzienlijk wordt beïnvloed door de verdeling en inhoud van de fasen. Vooral het martensietpercentage beïnvloedt de verdeling van spanning en rek als gevolg van interacties van de tweede orde. Anderzijds is het effect van martensietdistributie meer uitgesproken op de initiële hardingssnelheid van de RVE. Ten tweede werd na vergelijking van locaties van experimenteel waargenomen schade-incidenten en numerieke incidenten gezien dat een op plastische arbeid gebaseerde schade-indicator geschikt is voor het voorspellen van schadelocaties en van vatbaarheid voor schade van de microstructuur. Het bleek dat een toename van het martensietgehalte de kans op schade in zowel ferriet als martensiet verhoogt, terwijl een gelaagde morfologie meer schade in de martensietfase veroorzaakt. Ten slotte werd vastgesteld dat de evolutieeigenschappen van holtes sterk afhankelijk zijn van hun locaties, de omgeving, met andere woorden de lokale spannings- en rektoestand. Bovendien volgt de evolutie ook geen monotone trend. Interessant is dat in de simulaties tijdens trekvervorming enkele reeds bestaande holtes krimpen, wat een indicatie is van lokale druk- of afschuifbelasting. Dit toont ook de variatie van de lokale toestand ten opzichte van de gemiddelde voorgeschreven toestand. In het algemeen wordt een hogere groeisnelheid van de holtes waargenomen voor die holtes die zich tussen martensiet bevinden dan de andere holtes. 


\section{Contents}

Summary $\quad$ v

Samenvatting vii

1 Introduction $\quad \mathbf{1}$

1.1 Damage in dual phase steels . . . . . . . . . . . . . . . . 1

1.2 Objective of this thesis . . . . . . . . . . . . . . . 3

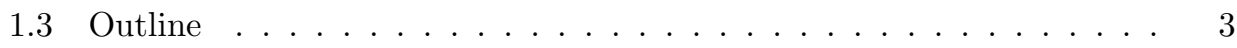

1.4 Notation . . . . . . . . . . . . . . . . . . . . . 4

2 Ductile damage in metals $\mathbf{5}$

2.1 Ductile damage mechanisms . . . . . . . . . . . . . . 5

2.1.1 General microstructural characteristics . . . . . . . . . . 5

2.1.2 Ductile damage mechanisms in dual phase steels . . . . . . . 7

2.2 Damage models . . . . . . . . . . . . . . . . . . . 10

2.2.1 Gurson-Tvergaard-Needleman model . . . . . . . . . . . . . . . 10

2.2.2 Lemaitre damage model . . . . . . . . . . . . . . . . . . . . . . . 11

2.2.3 Representative volume elements . . . . . . . . . . . . . . 12

2.3 Concluding remarks . . . . . . . . . . . . . . . . . . 14

3 Plastic strain heterogeneity in DP600 steels $\quad 15$

3.1 Introduction . . . . . . . . . . . . . . . . . . . . 15

3.2 Experimental Methods . . . . . . . . . . . . . . . . . . 17

3.2.1 Tensile tests . . . . . . . . . . . . . . . . 17

3.2.2 Electron backscatter diffraction analysis . . . . . . . . . . 18

3.3 Results and discussion . . . . . . . . . . . . . . . . . 21

3.3.1 Evolution of grain reference orientation deviation . . . . . . . 21

3.3.2 Evolution of grain orientation spread . . . . . . . . . . . . 21

3.3.3 Evolution of geometrically necessary dislocation density . . . . 24

3.3.4 Plastic heterogeneity around voids . . . . . . . . . . . . 28

3.4 Conclusions . . . . . . . . . . . . . . . . . . . 31 
4 Damage mechanisms in DP600 steel 33

4.1 Introduction . . . . . . . . . . . . . . . . . . . 33

4.2 Experimental details . . . . . . . . . . . . . . . . . . . . . . . . . . . . . . . . 34

4.2.1 General characteristics of DP600 sheet . . . . . . . . . . . . 34

4.2 .2 Interrupted tensile tests . . . . . . . . . . . . . . . . . . . . . . . . . . 35

4.2.3 Metallographic investigations . . . . . . . . . . . . . . . . 39

4.2 .4 Image processing . . . . . . . . . . . . . . . . . . . . . . . . . . . . . . . . . .

4.3 Results and discussion . . . . . . . . . . . . . . . . . . . . . . . . . . . . . .

4.3.1 Damage mechanisms . . . . . . . . . . . . . . 41

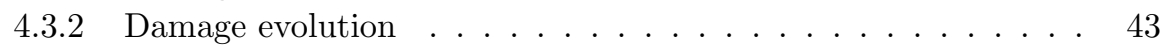

4.3 .3 Void distribution . . . . . . . . . . . . . . . . . . . 46

4.4 Conclusions . . . . . . . . . . . . . . . . . . . . . 51

5 Modelling Framework: Strain gradient enhanced crystal plasticity 53

5.1 Introduction . . . . . . . . . . . . . . . . . . 53

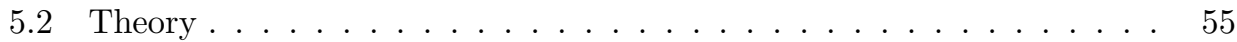

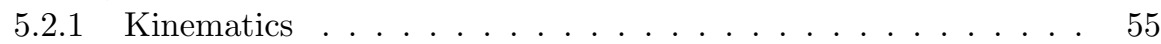

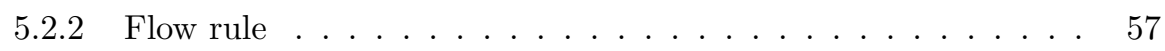

5.2 .3 Hardening rule . . . . . . . . . . . . . . . . . 58

5.2.4 GND density: Strain gradient enhancement . . . . . . . . . 59

5.3 Implementation . . . . . . . . . . . . . . . . . . . 60

5.3.1 Rate-independent crystal plasticity . . . . . . . . . . . . . 60

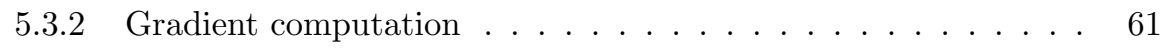

5.3.3 Global solution scheme . . . . . . . . . . . . . . 63

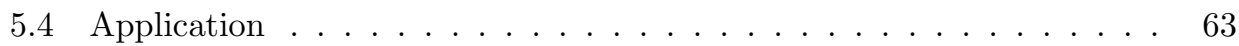

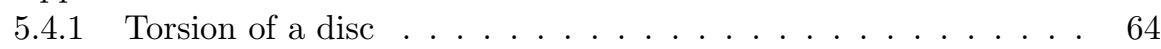

5.4 .2 Grain size strengthening . . . . . . . . . . . . . . 69

5.5 Conclusions . . . . . . . . . . . . . . . . . . . . 75

6 Damage initiation in dual phase steels $\quad 77$

6.1 Introduction . . . . . . . . . . . . . . . . . . 77

6.2 Microstructures and material properties . . . . . . . . . . . . 78

6.3 Results and discussion . . . . . . . . . . . . . . . . . 79

6.3.1 Partitioning of stress and strain . . . . . . . . . . . . 79

6.3.2 Comparison of damage indicators for martensite . . . . . . . . 82

6.3.3 Comparison of damage indicators for ferrite . . . . . . . . . 86

6.4 Conclusions . . . . . . . . . . . . . . . . . . . . . . 92

7 Damage evolution in crystals $\quad 93$

7.1 Introduction . . . . . . . . . . . . . . . . . . . 93

7.2 Void evolution in single phase polycrystals . . . . . . . . . . . . . 95

7.2.1 Effect of grain orientation on void evolution . . . . . . . . . 98

7.2.2 Effect of grain size on void evolution . . . . . . . . . . . . . . 101

7.2.3 Effect of initial void size on void evolution . . . . . . . . . . . . 103

7.3 Void evolution in dual phase steel . . . . . . . . . . . . . . . 105 


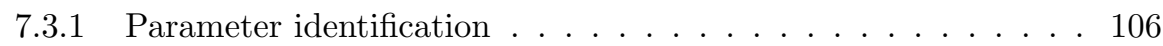

7.3.2 Evolution of dislocation densities . . . . . . . . . . . . . . . 109

7.3.3 Evolution of voids . . . . . . . . . . . . . . . . . . 114

7.3.4 Evolution of voids formed by cracking of martensite . . . . . . 116

7.4 Conclusions . . . . . . . . . . . . . . . . . . 117

8 Conclusions and Recommendations $\quad 119$

$\begin{array}{ll}\text { A Slip systems } & 123\end{array}$

$\begin{array}{lr}\text { B UMAT } & 127\end{array}$

$\begin{array}{lrl}\text { C USDFLD } & 129\end{array}$

$\begin{array}{ll}\text { Nomenclature } & 131\end{array}$

$\begin{array}{ll}\text { References } & 133\end{array}$

$\begin{array}{lr}\text { Research deliverables } & 147\end{array}$

$\begin{array}{lr}\text { Acknowledgments } & 149\end{array}$ 



\section{Introduction}

\subsection{Damage in dual phase steels}

For centuries, various types of steels have been indispensable materials that responded to the needs of humankind in a vast range of application fields such as transportation, infrastructure and medicine. The knowledge about controlling the properties of steels has played an important role in facing and overcoming the challenges of the modern life.

Environmental issues and sustainability concerns are pushing all industries to produce greener and more sustainable products. In engineering applications, these concerns show themselves in producing lighter structures with stronger materials. Automotive industry, for example, demands new types of steel grades, which have higher strength, better formability and high toughness. Consequently, total mass reduction would be achieved without sacrificing strength and crash-worthiness, resulting in more environmentally friendly vehicles. In this perspective, advanced high strength steels play a crucial role in structural applications due to their combination of mechanical properties such as high strength to weight ratio, high tensile strength to yield ratio and excellent formability. Furthermore, dual phase (DP) steels with the relative ease of controlling mechanical properties in predefined ranges, proved themselves to be promising candidates that satisfy the needs of the automotive industry [1-3].

Dual phase steels are named after their two main constituent phases, ferrite and martensite, see Figure 1.1. In addition, DP steels may contain a certain amount of bainite or retained austenite. Depending on the production parameters, martensite fraction and morphology can be controlled to obtain a specific grade of DP steel. The classification of this steel grade is made according to their ultimate tensile strength. As a general principle, in DP steels soft ferrite matrix surrounds hard martensite islands. Ferrite gives ductility to the alloy whereas martensite ensures the necessary strength. The combination of two mechanically diverse phases in such a complex microstructure gives the versatile properties of DP steels [4-7]. However, the presence of martensite also causes highly heterogeneous distribution of stress and strain in the microstructure 


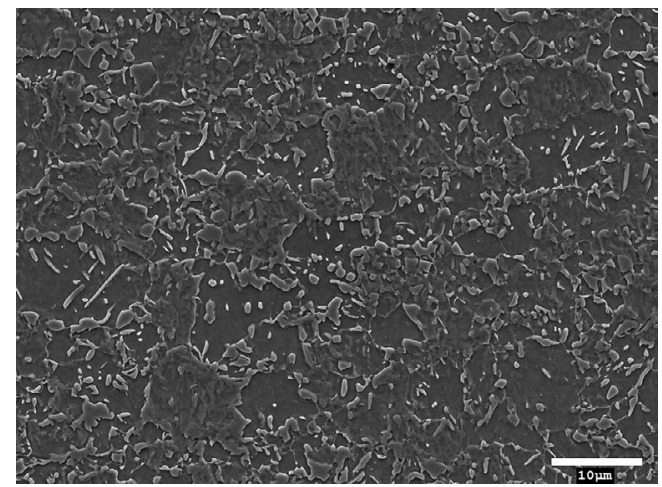

Figure 1.1: Typical microstructure of DP600 showing ferrite (dark gray) and martensite (light gray) phases.

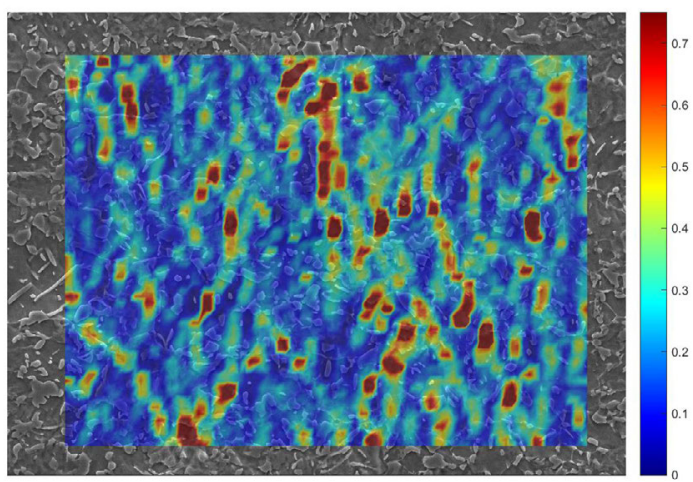

Figure 1.2: Distribution of strain $\left(\varepsilon_{11}\right)$ in a DP600 steel when it is elongated $18 \%$.

during deformation. Figure 1.2 shows such a distribution of $\varepsilon_{11}$ in a DP600 steel that was elongated up to the point where diffuse necking starts. It can be seen that there are locations where the strain reaches more than $70 \%$ while the average is about $18 \%$. Understanding and predicting the local conditions, such as stress and strain, play an important role in increasing the applicability of DP steels, because these conditions play an important role in hardening and failure characteristics.

Sheet metal forming processes are extensively used in industry to form a certain design shape, which would be more costly if the part was cast or machined. Sheet metal forming involves plastic deformation of the metal to the desired shape and during this process a large variety of stress states are generated throughout the sheet. In dual phase steels, the martensite content and distribution have significant effects on the variation of local stress state and the local stress intensities. The evolution of ductile damage in dual phase sheets is one of the critical factors which limits the formability and reduces the load-carrying capacity of the final product. Ductile damage, which is a phenomenon coupled with plastic deformation, occurs by nucleation of voids in 
local high strain regions and evolves through growth of the voids and coalescence of the voids eventually leading to macroscopic failure.

As the materials get more complex and product design becomes more demanding, the importance of the role of numerical simulations in manufacturing and microstructure design increases. There have been significant advancements in the numerical tools (e.g. Finite Element Method) used for simulating mechanical response during forming operations. However, the predictive capability of these models are limited by the underlying physics that is included in the constitutive behavior of the material. By employing physically-based models, virtual testing of the material and the manufacturing processes become possible even at the stage of microstructural design.

\subsection{Objective of this thesis}

The aim of this research is to unravel the effect of microstructural features on the damage behavior of dual phase steels. This is carried out by performing experimental and numerical analysis. The experimental investigations focus on the evolution of heterogeneity in plastic deformation at microstructural level and the resulting evolution characteristics of geometrically necessary dislocation density, characterization of damage mechanisms and distribution of damage incidents. The numerical analysis aims to understand and relate the effect of microstructural features such as grain morphology, phase distribution, second phase content to evolution of stress and strain fields, damage nucleation sites and evolution of damage incidents by using a micromechanical framework that can inherently capture the physical aspects of microstructural features.

\subsection{Outline}

Chapter 2 gives a short overview of the available literature on the topic for advanced high strength steels. In this chapter, important aspects such as: damage mechanisms and characteristics, and different modeling approaches are reviewed.

In Chapter 3, heterogeneity of plastic deformation and evolution of geometrically necessary dislocation density in a commercial dual phase steel is investigated by means of interrupted tensile tests and subsequent electron backscatter diffraction analysis. The results are quantified in terms of misorientation relationships.

In Chapter 4 active damage mechanisms and their evolution in a commercial dual phase steel are investigated experimentally by interrupted tensile tests. The investigations are conducted in both rolling and transverse directions. Subsequently, analysis of the damage mechanisms and void evolution was characterized by image analysis of micrographs.

In Chapter 5, the theory and implementation of a rate-independent crystal plasticity model and the strain gradient enhancement are presented. First, the kinematic model of crystal plasticity and geometrically necessary dislocation density is elaborated. 
Next, the details of the implementation of the framework are introduced. Finally, two example problems, torsion of a disc and grain size strengthening, are illustrated. These problems show the strain gradient effects resulting from the structural gradient of strain as well as the gradient that is caused by the inhomogeneous microstructural deformation.

In Chapter 6, the developed framework is used to characterize and investigate the effect of martensite distribution and content on the stress and strain partitioning in dual phase steels. Moreover, various damage indicators are compared in order to inspect the damage prone features in RVEs.

In Chapter 7, void growth characteristics in single and dual phase microstructures are investigated within the developed strain gradient enhanced crystal plasticity framework. The effects of grain orientation, grain size, void size, phase distribution morphology and damage location are elaborated by using RVE studies.

Finally, in Chapter 8, the conclusions drawn from this study are summarized and recommendations for future research are given.

\subsection{Notation}

In the thesis, following notations are consistently used: $1^{\text {st }}$ order tensors and vectors are shown in bold face and lowercase letters $(\mathbf{a}), 2^{\text {nd }}$ order tensors and matrices in bold face and uppercase letters $(\mathbf{A})$ and $4^{\text {th }}$ order tensors with blackboard bold face and uppercase letters $(\mathbb{A})$. The single contraction of tensors is represented by a dot $\left(\mathbf{A} \cdot \mathbf{b}=A_{i j} b_{j}\right)$, and double contraction by a colon $\left(\mathbf{A}: \mathbf{B}=A_{i j} B_{i j}\right)$. The dyadic (tensor) product is represented by the $\otimes \operatorname{sign}\left(\mathbf{C}=\mathbf{a} \otimes \mathbf{b}, C_{i j}=a_{i} b_{j}\right)$. The gradient operator is defined as $\nabla(\{\bullet\})=\frac{\partial(\{\bullet\})}{\partial x_{i}} \mathbf{e}_{i}$ 


\section{Ductile damage in metals}

This chapter focuses on the general aspects of damage in dual phase steels. It starts by giving an overview of ductile damage processes. Subsequently, damage evolution for dual phase steels is elaborated. In the final section, some common modeling approaches are introduced in order to give an overview of the current literature. The more specific state-of-the art literature is presented in the introduction section of each chapter.

\subsection{Ductile damage mechanisms}

\subsubsection{General microstructural characteristics}

Ductile damage in metals and alloys usually progresses in three stages. Firstly micro voids nucleate at $2^{\text {nd }}$ phase particles and inclusions $[8,9]$. Typically, this occurs by two mechanisms: decohesion of particles from the matrix or fracturing of particles. Later, the nucleated voids grow with plastic deformation. Finally, void coalescence takes place and leads to an accelerated degradation in the load-bearing capacity and to macroscopic failure. Figure 2.1 shows schematically the stages of damage evolution, from void nucleation to coalescence; deformation increases from left to right .

There are several parameters that influence the void nucleation mechanism, such as mechanical properties of the matrix (yield strength, hardening exponent), stiffness, shape of the $2^{\text {nd }}$ phase or particle, loading axis and stress state. Depending on these conditions, the void nucleation mechanism shifts from decohesion to cracking or vice versa. In the extensive review of Benzerga et al. [10], sensitivity of the dominant mechanism on these parameters are summarized qualitatively in Table 2.1.

Void growth is an intermediate step between void nucleation and coalescence. Depending on the material properties and loading conditions, the nucleated voids can grow significantly in size before coalescence. Two modes of void growth are reported in literature. In the first mode, hydrostatic stress dominates the growth and 


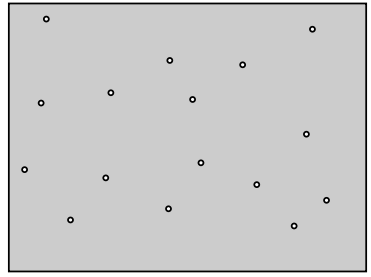

(a) Nucleation

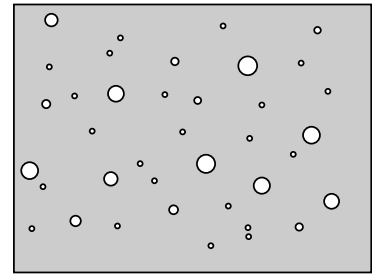

(b) Growth

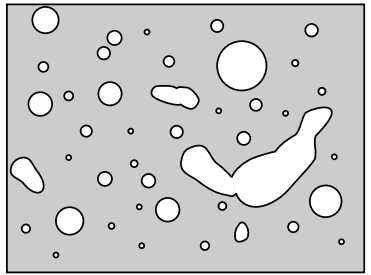

(c) Coalescence

Figure 2.1: Schematic illustration of ductile damage evolution gray color represents the matrix and white colored regions are voids.

Table 2.1: Trends in void formation mechanism [10].

\begin{tabular}{cccc}
\hline Parameter & Type & \multicolumn{2}{c}{ Trend } \\
$(\nearrow)$ & & Decohesion & Cracking \\
\hline Matrix yield strength & & $\nearrow$ & $\nearrow$ \\
Matrix hardening exponent & $\searrow$ & $\nearrow$ \\
Particle elongation & & $\searrow$ & $\nearrow$ \\
Particle stiffness & & $\nearrow$ & $\nearrow$ \\
Material orientation & rolling & $\searrow$ & $\nearrow$ \\
Stress triaxiality & transverse & $\nearrow$ & $\searrow$ \\
\hline
\end{tabular}

yields a more or less spherical void shape [11]. In the second mode, the growth takes place along the major axis of the principal component of stress and yields more penny shapes voids [12]. Moreover, the work of Crépin et al. [13] has also shown that void growth is affected by the crystal structure. In their work, they have observed tubular shaped hexagonal growth of voids for a hexagonal closed packed crystal structure.

When a considerable amount of void growth occurs in ductile materials, voids start to interact with each other, leading to void coalescence. Void coalescence is accepted as a transition from the relatively stable plastic deformation controlled growth to a localized mode. Void coalescence commonly happens by 3 different modes. These modes, in order of importance, necking of the intervoid ligament (internal necking), coalescence on a micro shear band (void sheeting) and necklace type coalescence along the loading axis [10,14-17]. Figure 2.2 shows the scanning electron micrographs of different coalescence mechanisms [10]. In the first mode - internal necking plastic deformation concentrates in the ligament between the two voids, causing the ligament to form a neck. This mode usually occurs when two voids are geometrically aligned perpendicular to the loading axis. In the second mode — void sheeting - 
shear localization occurs between the two voids. Formation of secondary voids in the shear band is also common for this type of coalescence. The third mode necklace coalescence - occurs when closely spaced voids are formed parallel to the main loading axis. This leads to formation of long columnar voids and looks like delamination. This specific type of coalescence is frequently reported in the presence of elongated intrusions or $2^{\text {nd }}$ phase particles.

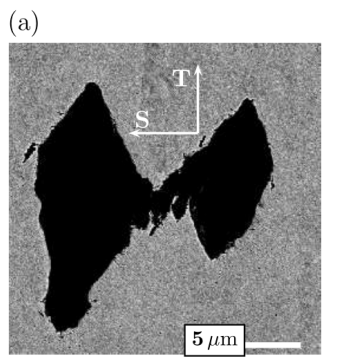

(b)

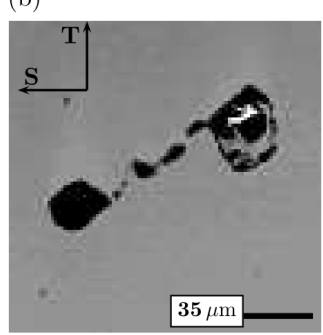

(c)

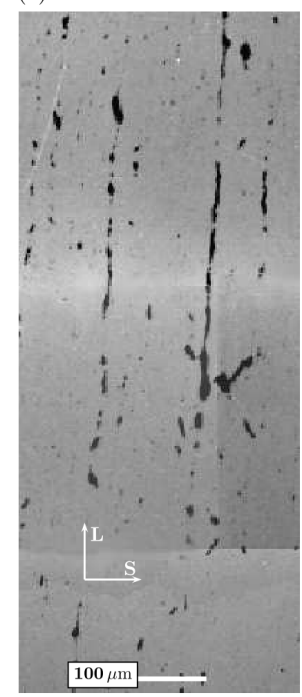

Figure 2.2: Different void coalescence mechanisms. (a) Necking of intervoid ligament, (b) coalescence in a micro-shear band and c) necklace coalescence. Major loading axis is vertical for all cases [10].

\subsubsection{Ductile damage mechanisms in dual phase steels}

In recent years, there have been many studies focusing on the damage evolution characteristics (active damage mechanisms, void growth, failure) of ferriticmartensitic dual phase (DP) steels. This is mainly due to the increasing popularity of this material in the automotive industry, which involves sheet metal forming operations.

In DP steels, void nucleation occurs mainly by cracking of martensite islands or decohesion at ferrite and martensite interfaces [7, 18-23]. On the other hand, conditions that promote one void initiation mechanism over the other are still ambiguous and not quantitatively characterized. In order to know the ductility limits and estimating load-bearing capacity left after the forming operations requires a precise understanding of the active damage mechanisms.

The ambiguity of active damage mechanisms is mainly a result of the variations in microstructural and mechanical properties which depend on martensite content, 


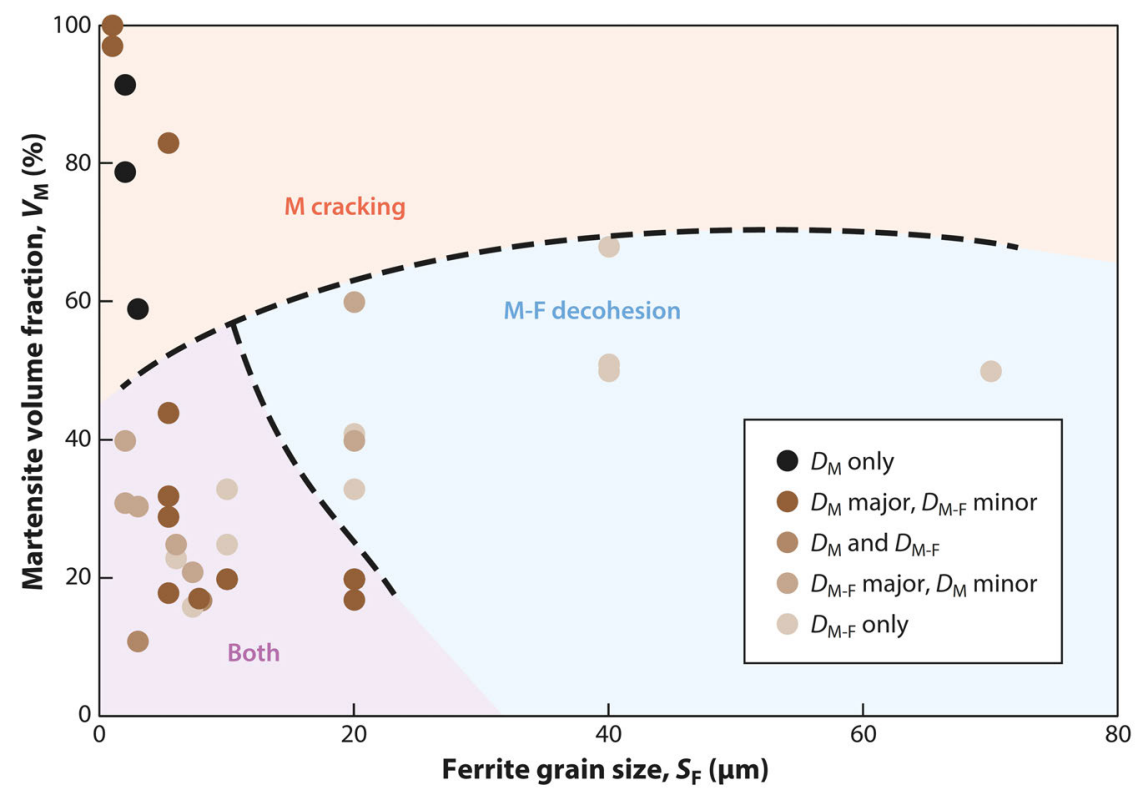

Figure 2.3: Active damage mechanisms with respect to martensite fraction $\left(\mathrm{V}_{\mathrm{M}}\right)$ and ferrite grain size $\left(\mathrm{S}_{\mathrm{F}}\right)$. $\mathrm{D}_{\mathrm{M}}$ is used for damage nucleation by martensite cracking and $\mathrm{D}_{\mathrm{M}-\mathrm{F}}$ is used for damage initiation by decohesion [7].

martensite morphology, martensite distribution, chemical composition of the phases and grain size of ferrite. In a recent review by Taşan et al. [7], the dominant damage mechanisms in DP steels with respect to martesite content $\left(\mathrm{V}_{\mathrm{M}}\right)$ and ferrite grain size $\left(\mathrm{S}_{\mathrm{F}}\right)$ are shown in Figure 2.3. From the figure, we can see that with increasing martensite content, cracking of martensite becomes the dominating damage mechanism, which is related to the formation of connecting martensite island/bands. In contrast, increase in the ferrite grain size changes the dominant mechanism to decohesion. For the industrially relevant cases, both damage mechanisms are active and it can be seen that the dominating damage mechanism changes from study to study.

In a recent comprehensive study, Hoefnagels et al. [21] compare the damage mechanisms in DP steels with various microstructural features under different loading conditions, namely uniaxial, biaxial and plane strain tension. In their study, three different DP microstructures are generated by heat treatment operations with different grain sizes and martensite contents. In addition, they have kept the carbon percentages of the martensite at the same value for all the microstructures. Two of these microstructures contain $33 \mathrm{vol} \%$ martensite but one has a finer grain size. The last microstructure is composed of 41 vol\% martensite. In their study, they distinguished five different damage mechanisms, which are damage at ferrite grain boundaries $\left(\mathrm{D}_{\mathrm{FGB}}\right)$, ferrite grain interiors $\left(\mathrm{D}_{\mathrm{FGI}}\right)$, around inclusions $\left(\mathrm{D}_{\mathrm{INC}}\right)$, martensite-ferrite interface $\left(\mathrm{D}_{\mathrm{INT}}\right)$ and damage formation by martensite cracking 


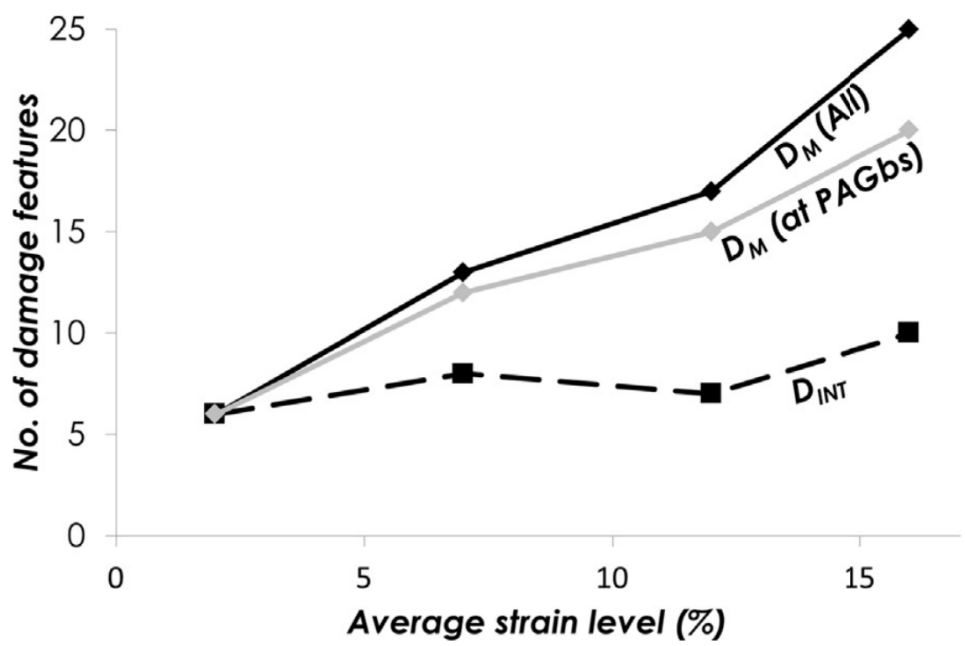

Figure 2.4: Variation in the number of damage incidents corresponding to different mechanisms with increasing average strain [26].

$\left(\mathrm{D}_{\mathrm{MC}}\right)$. The authors observed void nucleation in the early stages of deformation and identified $\mathrm{D}_{\mathrm{MFI}}$ as the dominant damage mechanism for all loading types while $\mathrm{D}_{\mathrm{MC}}$ became important only in biaxial tension. Moreover, more damage initiation occured with decreasing grain size under biaxial tension.

Banding of martensite islands is another feature which is frequently seen in DP steels. This occurs due to interdendritic segregation of alloying elements like Mn, Si and $\mathrm{Cr}$ during the solidification process. The subsequent hot rolling, elongates the grains resulting in banded segregated zones where the carbon activity is different. This causes variation in the Ac3 temperature leading to a banded microstructure [24]. The effect of a banded structure with a thick martensite band at the middle on damage behaviour was investigated by tensile tests in the study of Avramovic-Cingara et al. [18]. They found a higher damage content at the center line corresponding to the location of the thick martensite band. Moreover, they have investigated the active damage mechanisms and identified martensite cracking as the most important mechanism, contradicting the work of Hoefnagels [21].

In a pioneering work, Archie et al. [25] identify damage mechanisms in a DP steel with $30 \mathrm{vol} \%$ martensite under monotonic tensile loading. The authors manage to separate and identify damage incidents occuring at the prior austenite grain boundaries (PAGbs). Figure 2.4 shows the increase in the number of damage incidents with increasing global strain. Accordingly, damage initiation occurs at the very early stages of plastic deformation. Damage at PAGbs is the most frequently observed mechanism, which was attributed to the low fracture strength of PAGbs due to high Mn segregation. 


\subsection{Damage models}

In this section, we introduce three of the most frequently used approaches in order to model damage evolution in ductile solids. The first model is a micromechanical based dilatational plasticity model in which a homogenization approach is adopted to calculate the effect of void fraction on the flow strength. The second model on the other hand, adopts a phenomenological approach which relates a damaged body to an imaginary undamaged counterpart. In the third approach, damage is directly modeled in a representative volume element and the average response is simulated.

\subsubsection{Gurson-Tvergaard-Needleman model}

The Gurson-Tvergaard-Needleman (GTN) model is frequently used in order to model the failure of ductile metals. Gurson [27] has developed an approximate yield criteria and flow rule for porous materials which takes into account the hydrostatic stress in plastic yield and void growth. Gurson's model was first developed for a spherical void in a unit cell considering only void growth but not coalescence and nucleation. The yield function as proposed by Gurson is as follows:

$$
\Phi=\left(\frac{\sigma_{\mathrm{eq}}}{\sigma_{\mathrm{y}}}\right)^{2}+2 f \cosh \left(\frac{-3 \sigma_{\mathrm{m}}}{2 \sigma_{\mathrm{y}}}\right)-\left(1+f^{2}\right)
$$

where $\sigma_{\text {eq }}$ is the equivalent stress, $\sigma_{\mathrm{m}}$ is the hydrostatic part of stress tensor, $\sigma_{\mathrm{y}}$ is the initial yield stress and $f$ is the void fraction which is the ratio of the total volume of all cavities to the volume of the body.

The model was later modified and extended by Needleman and Tvergaard to include the effects of void nucleation and coalescence [28]. With this modification, new model paramenters $\left(\mathrm{q}_{1}, \mathrm{q}_{2}\right.$ and $\left.\mathrm{q}_{3}\right)$ and effective void fraction $\left(f^{*}\right)$ was added to define the yield function as:

$$
\Phi=\left(\frac{\sigma_{\mathrm{eq}}}{\sigma_{\mathrm{y}}}\right)^{2}+2 \mathrm{q}_{1} f^{*} \cosh \left(\frac{-3 \mathrm{q}_{2} \sigma_{\mathrm{m}}}{2 \sigma_{\mathrm{y}}}\right)-\left(1+\mathrm{q}_{3} f^{* 2}\right),
$$

where for a periodic array of voids the parameters are identified as $\mathrm{q}_{1}=1.25, \mathrm{q}_{2}=1$ and $\mathrm{q}_{3}=\mathrm{q}_{1}^{2}$ by Tvergaard and Needleman. The total void fraction $f$ is used to calculate the effective void fraction $f^{*}$ as:

$$
f^{*}(f)= \begin{cases}f & \text { for } f \leq f_{\mathrm{c}} \\ f_{\mathrm{c}}+\kappa\left(f-f_{\mathrm{c}}\right) & \text { for } f>f_{\mathrm{c}}\end{cases}
$$

with:

$$
\kappa=\frac{f_{\mathrm{u}}^{*}-f_{\mathrm{c}}}{f_{\mathrm{f}}-f_{\mathrm{c}}}
$$


where $f_{\mathrm{c}}$ is the critical volume fraction when coalescence starts, $f_{\mathrm{f}}$ is the void fraction at macroscopic failure and $f_{\mathrm{u}}^{*}$ is the effective void fraction at macroscopic failure as $f_{\mathrm{u}}^{*}=1 / \mathrm{q}_{1}$. The effective void fraction allows the interactions between voids to be taken into account and prevent an unrealistic void fraction at failure which was experimentally observed [29]. Up to the critical level, $f_{*}$ increases linearly with $f$ and after the critical level, it increases exponentially causing a rapid shrinkage in the yield function.

In the GTN model the initial void fraction evolves both with void nucleation and growth which is expressed in rate form as:

$$
\dot{f}=\dot{f}_{\text {Growth }}+\dot{f}_{\text {Nucleation }}
$$

Here, the growth of voids is driven by the volumetric part of plastic strain rate as:

$$
\dot{f}_{\text {Growth }}=(1-f) \operatorname{tr}\left(\dot{\varepsilon}_{\mathrm{p}}\right),
$$

and void nucleation is related statistically to the evolution in the equivalent plastic strain rate $\dot{\bar{\varepsilon}}_{\mathrm{p}}$ as:

$$
\dot{f}_{\text {Nucleation }}=\frac{f_{\mathrm{N}}}{S_{\mathrm{N}} \sqrt{2 \pi}} \exp \left[-\frac{1}{2}\left(\frac{\bar{\varepsilon}_{\mathrm{p}}-\varepsilon_{\mathrm{N}}}{S_{\mathrm{N}}}\right)^{2}\right] \dot{\bar{\varepsilon}}_{\mathrm{p}}
$$

where $\varepsilon_{\mathrm{N}}$ is the mean equivalent void nucleation strain, $f_{\mathrm{N}}$ is the fraction of void nucleating particles and $S_{\mathrm{N}}$ is standard deviation of equivalent strain where void nucleation occurs.

\subsubsection{Lemaitre damage model}

Lemaitre's damage model is based on continuum damage mechanics (CDM) which describes continuous loss of material properties as void nucleation, growth and coalescence occurs. It doesn't explicitly define damage as presence of voids but indirectly uses the effect of the damage on the load bearing capacity of the body. Damage is expressed as a variable $D$, which is a scalar for isotropic damage and is a tensor for anisotropic damage [30-34]. The damage variable, $D$, shows the fraction of the degraded property (stress, strain or elastic modulus). This concept was first adopted by Kachanov et al. [30] in order to model evolution of creep damage. In this concept, the property in a damaged state is represented with its effective equivalence in the virtual undamaged state. In this section, variables of the effective virtually undamaged states are denoted with $\tilde{\bullet}$ where $\bullet$ is the counterpart of the property in the damaged state.

Figure 2.5 shows the main idea of effective stress concept, which is to express a damaged cross-section area containing discontinuities with a fictitious continuous area. The fictitious area, which is the net area is then written as the difference between the measured and damaged area of the cross-section:

$$
\delta \tilde{S}=\delta S-\delta S_{\mathrm{D}}
$$




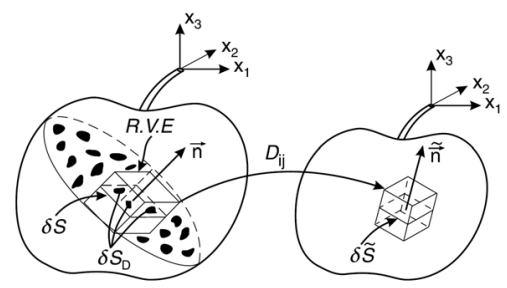

Figure 2.5: Damaged and virtual undamaged states [35].

where $\delta S$ is the total infinitesimal area, $\delta S_{\mathrm{D}}$ is the area occupied by the voids or microcracks and $\delta \tilde{S}$ is the effective net area.

For an isotropic case, where the damage variable $D$ is a scalar value $0 \leq D \leq 1$, effective stress is defined as:

$$
\tilde{\boldsymbol{\sigma}}=\frac{1}{1-D} \boldsymbol{\sigma}
$$

with

$$
D=\frac{\delta S_{\mathrm{D}}}{\delta S}=\frac{\delta S-\delta \tilde{S}}{\delta S}
$$

For an isotropic material the energy density release rate $(Y)$, the variable associated with $D$, is derived from the state potential as:

$$
Y=\frac{\sigma_{\mathrm{eq}}^{2} R_{\nu}}{2 \mathrm{E}(1-D)^{2}}
$$

with

$$
R_{v}=\frac{2}{3}(1+\nu)+3(1-2 \nu)\left(\frac{\sigma_{\mathrm{m}}}{\sigma_{\mathrm{eq}}}\right)^{2}
$$

where $\sigma_{\text {eq }}$ is the equivalent stress, $\sigma_{\mathrm{m}}$ is the mean stress, $\mathrm{E}$ is the elastic modulus and $\nu$ is the Poisson ratio. When a critical equivalent strain is exceeded the damage evolution follows

$$
\dot{D}=\dot{\bar{\varepsilon}}_{\mathrm{p}}\left(\frac{Y}{\mathrm{M}}\right)^{\mathrm{b}}
$$

where $\mathrm{M}$ and $\mathrm{b}$ are material parameters and $\dot{\bar{\varepsilon}}$ is the equivalent plastic strain rate.

\subsubsection{Representative volume elements}

Representative volume elements are micro scale models which include the microstructural features of a material, such as constituent phases, voids, boundaries. The RVE models give the response of a material at a microscopic point. Therefore, these models are frequently employed in order to study the underlying physics of a phenomenon and calculate the average macroscopic response of the material point. In addition, they can be used in multi scale simulations where for each integration point the average response is calculated from the RVE. 
In recent years, RVEs have been used to study damage initiation in micro scale by many researchers $[19,36-39]$. Some of these works focus on representing the physics of deformation and some focus more on the statistical effects of distribution of the phases on the average mechanical response. In the study of Ramazani et al. [40], damage initiation in martensite was investigated by using the realistic microstructure of a dual phase steel. They used the XFEM approach with isotropic plasticity model to simulate cracking of martensite and validated their results with experiments, see Figure 2.6.
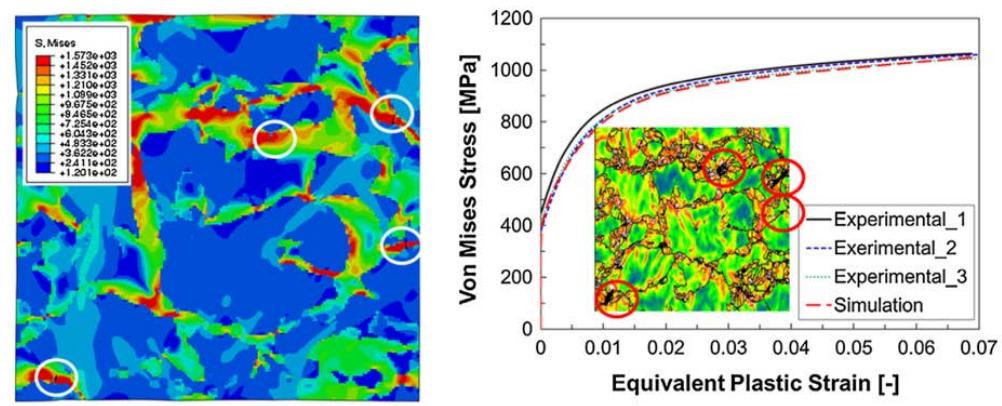

Figure 2.6: Microcrack formation in martensite (left), and comparison between experimental and simulated true stress-true strain curves (right), taken from Ramazani et al. [40].

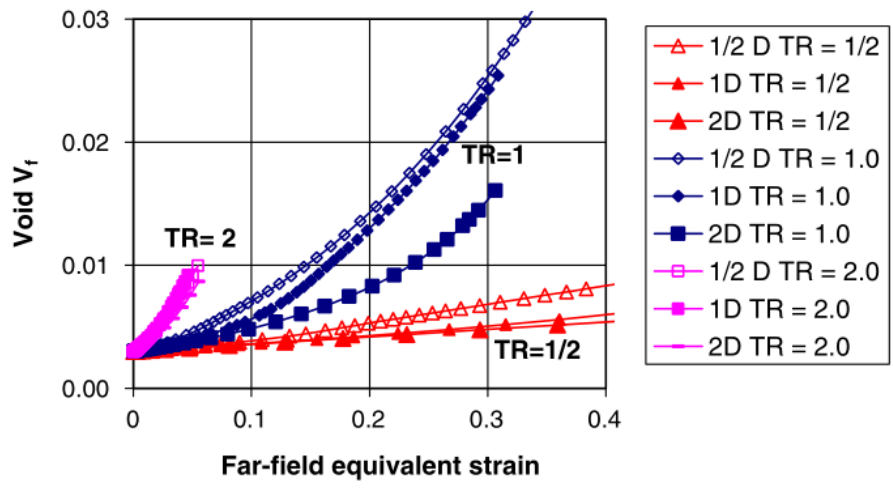

Figure 2.7: Evolution of void fraction with respect to stress triaxiality (TR) and , inter-void spacing (D) taken from Bandstra et al. [41].

Representative volume elements are also used in order to investigate void growth. This can be accomplished by employing unit cell calculations where the voids are modeled explicitly [29,41-43]. Such a study was conducted in the work of Bandstra et al. [42], where the authors considered the influence of void clusters on void growth. 


\subsection{Concluding remarks}

Ductile damage occurs in three steps — void nucleation, void growth and void coalescence - and after nucleation these steps occur simultaneously until fracture. The nucleation of voids occurs by cracking of an inclusion or at the boundary between the matrix and the inclusion. Local stress and strain states have significant effects on the evolution of damage.

There have been many studies to understand damage in dual phase steels. In general the trend in single phase materials is valid for these alloys, however, the presence of martensite, which has high mechanical contrast with ferrite, increases the complexity of deformation as well as variation in the local conditions. Thus, literature work on the topic of dominant damage mechanism in DP steels sometimes contradict each other.

Most damage models, e.g. Lemaitre and GTN, rely on an approximation of the void volume fraction inside the material. In the continuum damage mechanics approach the main goal is to determine the stress-strain response of the material for the given void content in a thermodynamically consistent framework. However, due to the lack of an underlying physical model for the evolution of void fraction this approach should be considered mostly as a phenomenological one.

Although the Gurson model and its derivatives (GTN) stem from a physical basis the parameters that are used to describe the nucleation, growth and coalescence of voids are statistical and are mostly based on fitting to experimental results.

Therefore, it can be concluded that for an accurate and physically based description of damage evolution in macroscale, the nucleation, growth and coalescence phenomena must be understood. The RVE approach seems to be the most promising method for this purpose since the evolution of strain and stress fields as well as the damage mechanisms can be physically taken into account. This allows understanding the evolution of damage throughout the complete deformation process and hence paves the way to a physical basis for macroscopic ductile damage models. 


\section{Plastic strain heterogeneity in DP600 steels}

This chapter investigates the heterogeneity of plastic deformation in a commercial dual phase steel by means of evolution of lattice orientations and their gradients. Interrupted tensile tests are conducted in the macroscopically uniform region of plastic deformation in a tensile test and electron backscatter diffraction (EBSD) analysis is carried out on the deformed samples. The EBSD data is evaluated in terms of grain reference orientation deviation (GROD), grain orientation spread (GOS) and kernel average misorientation (KAM) values. The misorientation measures, GROD and GOS, quantify the inhomogeneity of plastic deformation within a grain while KAM is used to quantify the geometrically necessary dislocations. Moreover, EBSD analysis is carried out in the vicinity of voids in order to establish a link to damage initiation.

\subsection{Introduction}

Inelastic deformation of polycrystalline materials is a non-uniform process which can be attributed directly to the presence of grains with different orientations, sizes and shapes. The discrete nature of crystallographic slip along preferred orientations and the dependence of activation of slip on the orientation cause anisotropic plastic response $[44,45]$. In addition, it is known that grain morphology directly effects the strength of metals [46-48]. The combination of these factors yields various stress states, stress and strain distributions in the microstructure even for a single phase polycrystalline material at the early stage of plastic deformation. Moreover, plastic deformation in DP steels is highly heterogeneous due to the presence of the $2^{\text {nd }}$ phase (martensite) which exhibits a mechanical behavior very diverse from ferrite and is distributed non-homogeneously in the microstructure.

In the last decade, electron backscatter diffraction analysis combined with scanning electron microscopy has become a powerful technique to characterize microstructural 
features in ((poly)crystalline materials [49-54]. This technique has been used by researchers in order to quantify the degree of plastic deformation or damage from the local changes in the lattice orientations [55-59]. However, most of the studies are limited to single phase materials.

Heterogeneity of plastic deformation in IF steels was studied by Allain-Bonasso et al. [56]. In their study, the relationship between grain orientation spread and plastic strain was investigated. It was shown that grain orientation spread increases with plastic deformation. Moreover, they observed that the GOS values of larger grains were greater than the smaller grains.

Kim et al. studied microtexture development in dual phase steels under equibiaxial tensile deformation [58]. They reported high strain values based on the darkness of band contrast values near the martensite islands. In addition, they found out by using KAM measurements that these regions also had higher GND density.

Recently, the effect of cyclic loading on the orientation relationships was investigated for Fe-3Si alloys [51]. The study of Schayes et al. showed that at low strain variations, $\Delta \varepsilon<0.7 \%$, grain reference orientation deviation, grain orientation spread and kernel average misorientation values did not reveal strain localization for single phase Fe3Si alloy. However, higher strain amplitudes yielded strain localization near grain boundaries [51]. Rui et al. investigated creep deformation induced grain lattice distortion for austenitic stainless steels [59]. They reported that for two independent creep conditions the GROD value was a linear function of creep strain.

The orientation gradients and geometrically necessary dislocations in dual phase steels were studied by Calcagnotto et al. [49]. They investigated the effect of volume expansion during transformation from austenite to martensite in two different ultrafine grained DP steels. They conducted both 2D and 3D EBSD analyses in order to investigate the orientation gradients. They found that orientation gradients were higher around martensite islands. Moreover, ferrite-martensite interfaces contained higher gradients than ferrite-ferrite boundaries. In their study, they also compared two methodologies (linear relationship with KAM value and construction of dislocation tensor) for calculating GND density and observed that both methods yield similar results.

In this chapter, we have investigated heterogeneity in plastic deformation distribution and the evolution of geometrically necessary dislocations (GNDs) in a DP600 steel sheet which was subjected to quasi-static tensile loading. For this purpose specimens were stretched in the rolling direction up to seven different stress levels. After deformation, orientation variations within the grains were quantified by using electron backscatter diffraction analysis. The evolution of lattice orientations was investigated by using grain reference orientation deviation (GROD), grain orientation spread (GOS) and the GNDs were quantified from the kernel average misorientation. Moreover, three different locations, that contain voids, were investigated in order to seek the origin of void nucleation. 


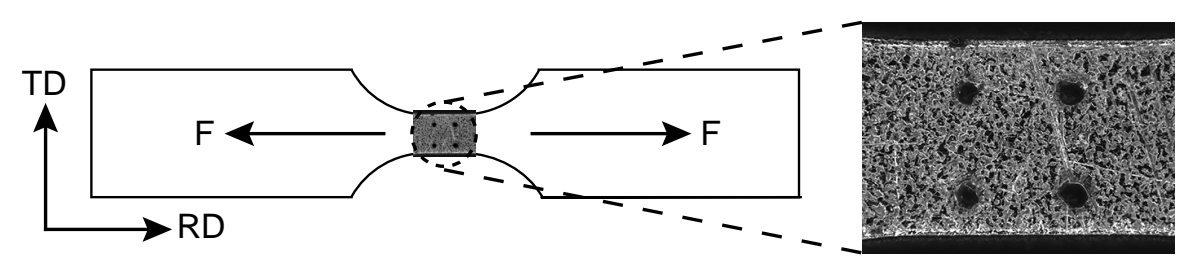

Figure 3.1: Deformation area and four silicone marks from which the strain is calculated. Direction of the applied load (F) is shown with arrows.

\subsection{Experimental Methods}

In this chapter, we investigate heterogeneity of plastic deformation in a dual phase microstructure by using electron backscatter diffraction (EBSD) analysis. Due to the plastic non-uniformity, the local orientations of points in a grain evolve differently from each other. This variation in the evolution of local grain orientations can be quantified by using grain reference orientation deviation (GROD), grain orientation spread (GOS) and kernel average misorientation (KAM) values, which are scalar indicators of non-uniformity of plastic strain and its gradient.

The material used in this study was a commercially available galvanized, coldrolled and annealed ferritic/martensitic DP600 steel sheet (Tata Steel, Ijmuiden, The Netherlands) which contains $0.09 \% \mathrm{C}, 0.5 \% \mathrm{Cr}, 1.9 \% \mathrm{Mn}, 0.06 \% \mathrm{Si}(\%$ wt) with a martensite content of $18 \pm 1.2 \mathrm{vol} \%$ determined by using three secondary electron images.

Interrupted tensile tests were conducted along the rolling direction at eight samples under quasi-static loading conditions. All of the samples were prepared from the same sheet. After deformation, EBSD measurements were realized from the normal direction of the sheet. Following two sections provide further details on the experimental methodology employed in this chapter.

\subsubsection{Tensile tests}

DP600 tensile test samples with a gauge geometry (length and width) of $3 \times 3 \mathrm{~mm}$ were prepared along the rolling direction, Figure 3.1. The samples were tested at room temperature with a $5 \mathrm{kN}$ capacity tension compression module (Kammrath \& Weiss GmbH, Germany) at $3 \mu \mathrm{m} / \mathrm{s}$ constant cross head speed corresponding to an initial strain rate of $6.5 \times 10^{-4} \mathrm{~s}^{-1}$. Eight samples were loaded to different engineering stress levels in the range of 500 to $690 \mathrm{MPa}$, which lie in the uniform plastic deformation regime. Macroscopic strains of specimens were calculated by correlating low magnification stereomicrographs of initial and final conditions by using 4 silicone paste marks that were applied on the samples prior to the tests by using a stencil. Figure 3.1 shows the area of interest, where deformation takes place, and the marks looking from the normal direction of the sheet. 


\subsubsection{Electron backscatter diffraction analysis}

Following the tensile deformation, samples were prepared for electron backscatter diffraction (EBSD) measurements. Prior to analyses, the surface of deformed samples was ground with 500 grit $\mathrm{SiC}$ paper and polished by using alumina suspension in the range of 9-1 $\mu \mathrm{m}$ and $0.04 \mu \mathrm{m}$ colloidal silica suspension (Struers Inc., US) as suggested by Vander Voort et al. [60].

All of the EBSD measurements were collected using a field emission scanning electron microscope (Jeol-SEM $7200 \mathrm{~F}$ Ltd, Japan) operating at $15 \mathrm{kV}$ accelerating voltage with $70^{\circ}$ tilting angle. The electron backscatter patterns (EBSP) were acquired at a step size of $70 \mathrm{~nm}$ with a $1344 \times 1024$ resolution EBSD detector and acquisition system (HKLNordlys, Oxford Instruments plc, UK) with an unbinned detector setting and indexed in the refined accuracy mode of AZtec. The analyses were carried out on a $50 \times 50 \mu \mathrm{m}^{2}$ square region from the center of the deformation region in order to investigate evolution of deformation heterogeneity. Moreover, four EBSD measurements were conducted around different voids. The measurement area was adjusted to cover enough surrounding to investigate the origin of void nucleation.

After the EBSD measurements, only the indexed data (pixels with an orientation and phase id) was post-processed in order to distinguish martensite from ferrite and calculate grain reference orientation deviation (GROD), grain orientation spread (GOS) and kernel average misorientation (KAM). The free MATLAB ${ }^{\circledR}$ toolbox MTEX was used for all post-processing operations [61].

Phase identification in EBSD relies on crystal structure and the lattice parameters. Due to the resemblance of crystal structure and lattice parameter of martensite and ferrite, EBSD software cannot differentiate between these phases during the data acquisition stage. Therefore, martensite is identified as ferrite. The gathered data has to be post-processed in order to correct phase identification.

In the literature, it was shown that grain averaged values of band slope or band
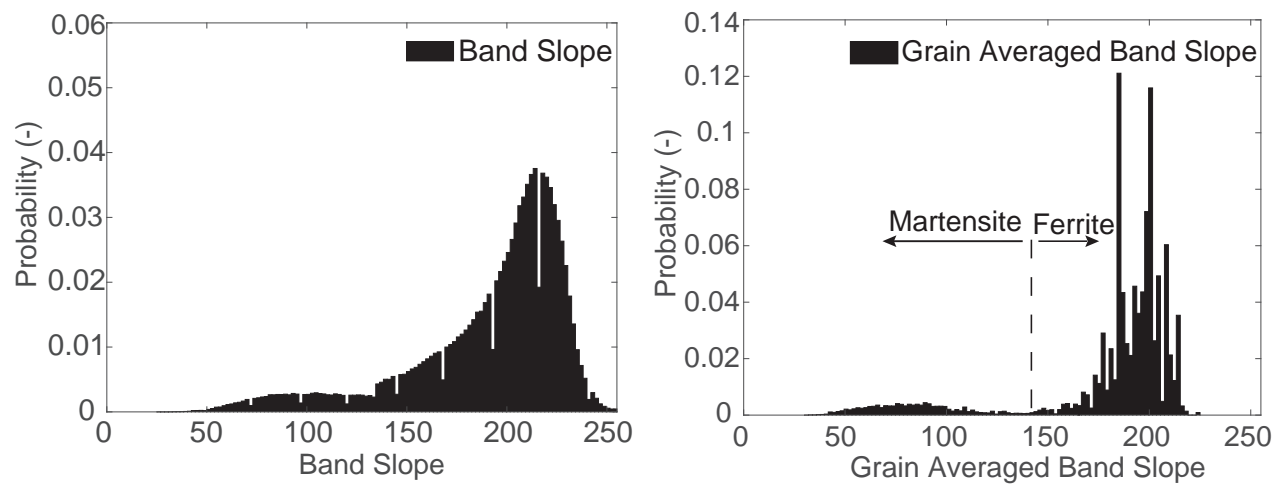

Figure 3.2: Distribution of band contrast (left) and grain averaged band slope (right) values. The critical grain averaged band slope value below which the grains are reindexed as martensite. 
contrast (image quality) gave more reasonable results in separating the phases than pixel based methods. Due to the internal stress present in martensite, the EBSD patterns of martensite have more diffuse Kikuchi bands. Thus, the band slope value is lower and martensite appears darker. Averaging band slope values for the grains enhances the procedure by preventing incorrect indexing of intragrain pixels [62]. Figure 3.2 shows the pixel based and the grain averaged band slope histograms. As can be seen in the grain averaged band slope histogram, there is a saddle point which can be used to differentiate the phases from each other. Moreover, grain area can also be used as a criterion in the analysis as suggested by Ryde et al. [63], due the difference between the size of martensite and ferrite phases.

In this study, we employed the grain averaged band slope methodology described above to differentiate between ferrite and martensite. Moreover, grains with an area smaller than $3.0 \mu^{2}$ were indexed as martensite regardless of its grain averaged band slope value. After re-indexing the phases, the average martensite content was calculated as $16.2 \pm 4.4$ vol\%, which is lower than the average value calculated from the micrographs with a higher standard deviation. There are two main reasons for this. Firstly, martensite yields lower quality or a highly distorted Kikuchi pattern such that the EBSD software does not index (calculate the orientation of) the pixel. This means that the pixels which are not indexed have a higher probability of belonging to martensite. Secondly, the measurement area $\left(50 \times 50 \mu \mathrm{m}^{2}\right)$ is small and due to the heterogeneous distribution of martensite it does not fully represent the martensite content. This also explains the higher standard deviation present.

The misorientation between two points can be expressed by using a scalar measure from the orientation matrix of the two points. This scalar misorientation angle $\Delta \theta_{i}^{j}$

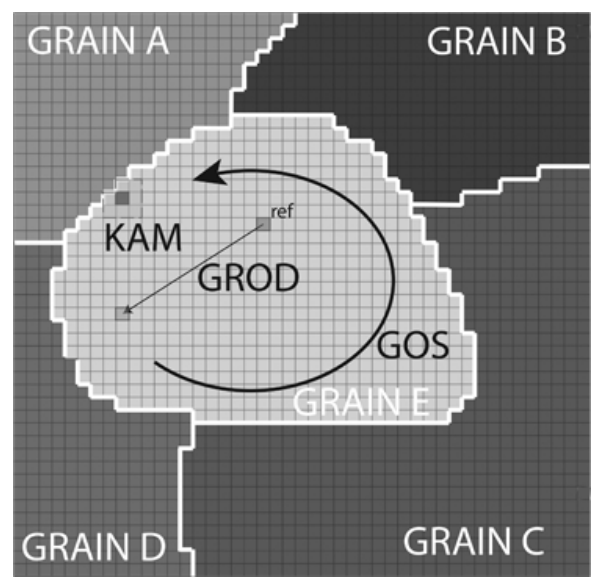

Figure 3.3: Schematic illustration explaining the calculation of grain reference orientation deviation (GROD), grain orientation spread (GOS) and kernel average misorientation (KAM) values. 
between the points $i$ and $j$ is calculated as:

$$
\Delta \theta_{i}^{j}=\cos ^{-1}\left(0.5\left[\operatorname{tr}\left(\mathbf{G}_{j} \cdot \mathbf{G}_{i}^{-1}\right)-1\right]\right),
$$

where $\mathbf{G}_{i}$ and $\mathbf{G}_{j}$ are the $2^{\text {nd }}$ order orientation tensors of points $i$ and $j$.

By using this definition, several quantities related to misorientation can be calculated. Figure 3.3 illustrates how the grain reference orientation deviation, grain orientation spread and kernel average misorientation are calculated.

Grain reference orientation deviation (GROD) is calculated by using:

$$
\mathrm{GROD}_{i}=\Delta \theta_{i}^{\mathrm{ref}}
$$

where $\Delta \theta_{i}^{\text {ref }}$ is the misorientation angle between the point $i$ and a reference point in the same grain. In this study the reference orientation is selected as the mean orientation of the grain [59].

Grain orientation spread (GOS) is the average value of grain reference orientation deviation within a grain and it is calculated as

$$
\operatorname{GOS}_{E}=\frac{1}{M} \sum_{i=1}^{M} \operatorname{GROD}_{i}
$$

where $M$ is number of points of the grain $E[51]$.

Both GROD and GOS show the deformation inhomogeneity in a grain. While GOS is a single value for a grain which quantifies the average non-uniformity, GROD defines the deviation from the average orientation for points in a grain. The evolution of these quantities is based on the difference between the rate of change of lattice orientation of the points in the grain. This can be expressed as the difference between local and plastic spin $\left(\mathbf{W}-\mathbf{W}_{\mathrm{p}}\right)$. If the deformation is microscopically uniform, the local texture would evolve in the same way for all the points such that the misorientation between the reference point (ref) and point $i$ would be equal to zero.

The kernel average misorientation of the point $i$ is calculated as:

$$
\mathrm{KAM}_{i}=\frac{1}{N} \sum_{j=1}^{N} \Delta \theta_{i}^{j}
$$

where $N$ is the total number of neighbors and $j$ is the neighbor of $i$. If point $j$ is not in the same grain as point $i$, it is not included in the averaging. We have taken into the first order neighbors with a maximum $\Delta \theta_{i}^{j}$ of $5^{\circ}$ in our calculations. In the literature, the KAM value is frequently employed in order to estimate the density of geometrically necessary dislocations since it calculates a local average gradient of orientation $[49,64-66]$. 


\subsection{Results and discussion}

\subsubsection{Evolution of grain reference orientation deviation}

In this section, grain reference orientation deviation in ferrite is investigated. As given in Equation (3.2), this value shows deviation of orientation of a point within a grain from the average orientation of a grain. The GROD value is a result of the difference in local evolution of lattice orientation within a grain.

Figure 3.4 shows the evolution of GROD values in the ferrite phase. Initially, in the undeformed state, grains in the analysis area of interest do not show high GROD values. Only around some martensite islands and near some ferrite grain boundaries, there are very local high GROD intensity zones. There is also one big grain which has high GROD values. This is due to the presence of a low angle boundary.

With increasing deformation, orientation deviation starts to increase from the grain boundaries and regions which are close to martensite islands. At a strain level of 0.06 , it is seen that high GROD values spread in to ferrite grains, leaving mostly the grain centers in low GROD values. At the maximum applied strain, only very small parts of the ferrite grains are left with low orientation spread and most of the grains contain high GROD values. Overall, the average GROD values of ferrite in the measurement areas increase from $1.26^{\circ}$ to $4.64^{\circ}$.

These results show that near the grain boundaries and the martensite islands (shown with white color in Figure 3.4), the orientation deviates more from the average of the grain.

\subsubsection{Evolution of grain orientation spread}

In this subsection, grain orientation spread (GOS) in ferrite is investigated. As given in Eq. (3.3) it is an average grain value. Therefore, GOS shows an average of the nonuniformity of texture evolution and heterogeneity in plastic deformation of the grain. Higher GOS values indicate a wider variation of grain orientation and a higher amount of plastic deformation heterogeneity. The evolution of GOS follows the evolution of GROD values. Figure 3.5 shows how GOS values of the grains change with increasing strain.

Initially, the orientation spread in the grains is very small. There is only one grain sample which has a high GOS value. This is due to the low angle boundary that divides the grain in two parts. These parts could be considered as two different grains as well. But since the orientation difference between the two parts was smaller than the grain boundary limit criterion of $5^{\circ}$ difference, they were indexed as one grain.

With increasing tensile strain, the spread in ferrite grains increases. The increase does not show a general pattern to conclude whether GOS increases mostly in small grains or the large grains, as observed in IF steel [56]. This can be attributed to the more complex stress and strain partitioning in DP steels. It is seen that there are some small grains as well as some large grain with high GOS values. 
$0.0^{\circ} \quad 5.0^{\circ}$

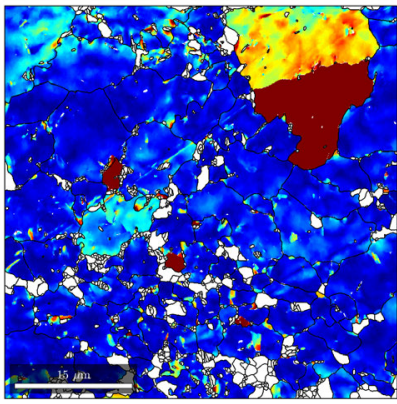

$\varepsilon_{11}=0 \%$

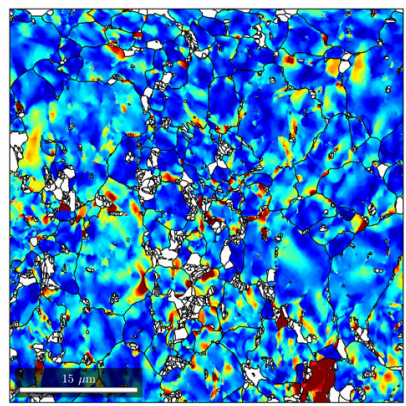

$\varepsilon_{11}=4.72 \%$

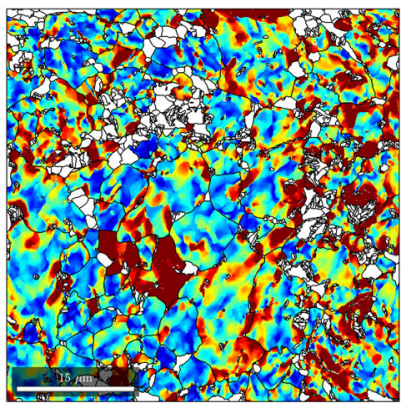

$\varepsilon_{11}=9.71 \%$

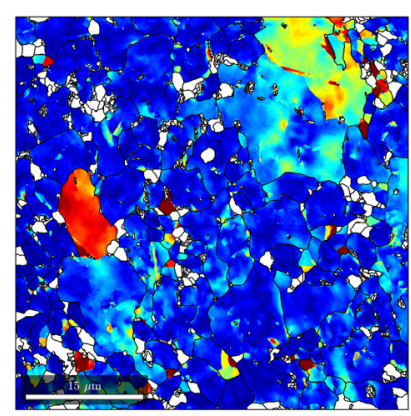

$\varepsilon_{11}=1.26 \%$

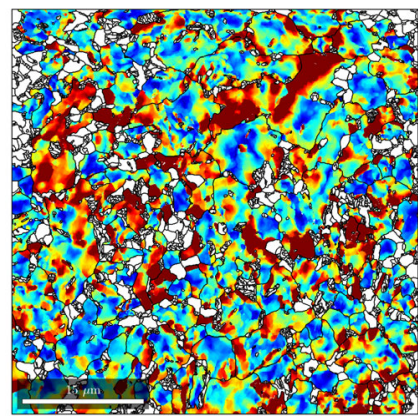

$\varepsilon_{11}=6.0 \%$

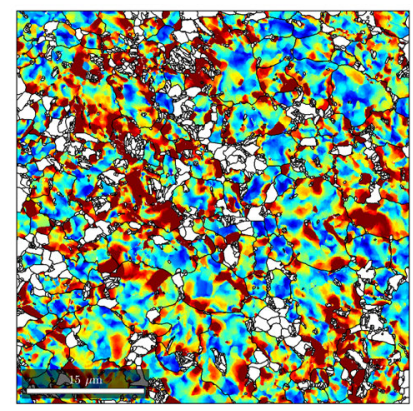

$$
\varepsilon_{11}=10.49 \%
$$

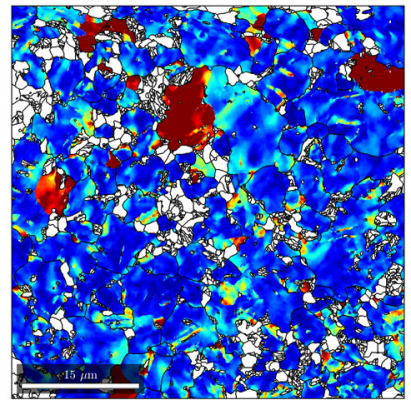

$\varepsilon_{11}=2.97 \%$

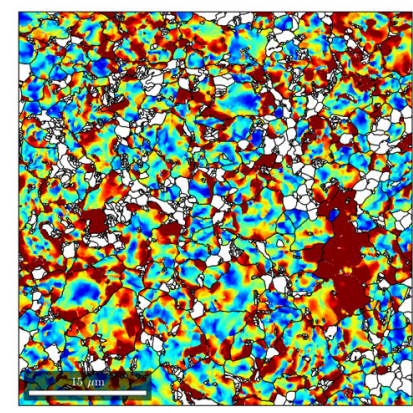

$\varepsilon_{11}=7.87 \%$

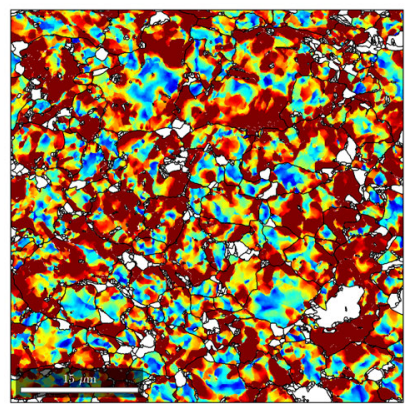

$\varepsilon_{11}=18.11 \%$

Figure 3.4: Evolution of GROD with increasing strain. White regions are martensite islands and black lines are boundaries. 
$0.0^{\circ}$

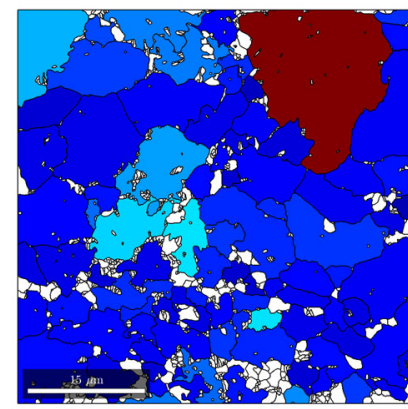

$\varepsilon_{11}=0 \%$

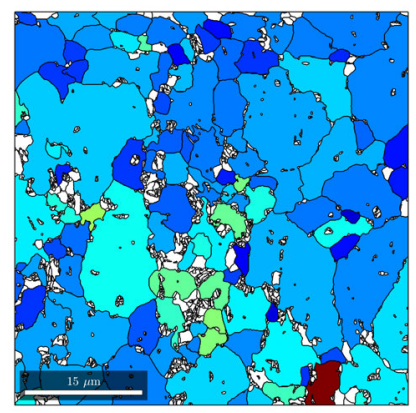

$\varepsilon_{11}=4.72 \%$

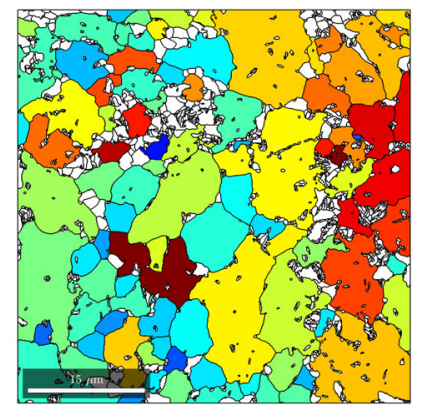

$\varepsilon_{11}=9.71 \%$

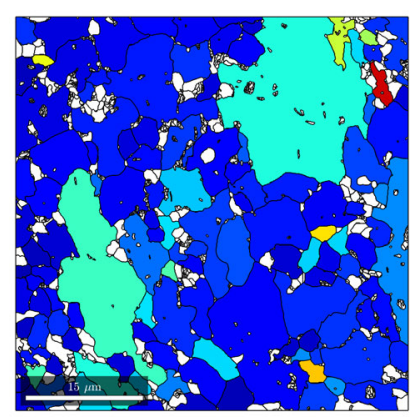

$\varepsilon_{11}=1.26 \%$

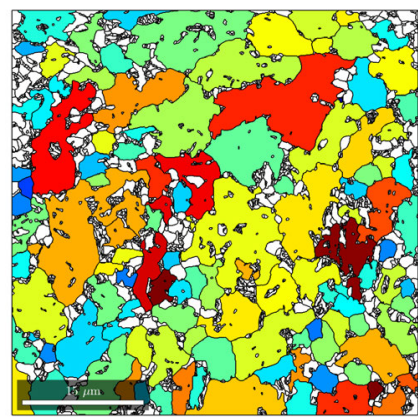

$\varepsilon_{11}=6.0 \%$

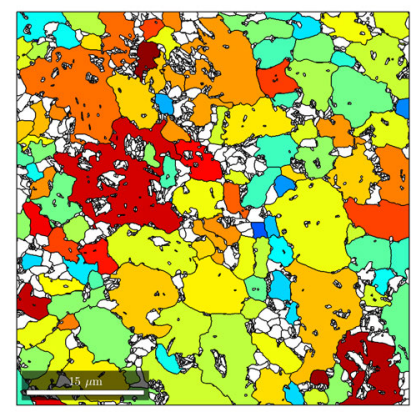

$\varepsilon_{11}=10.49 \%$

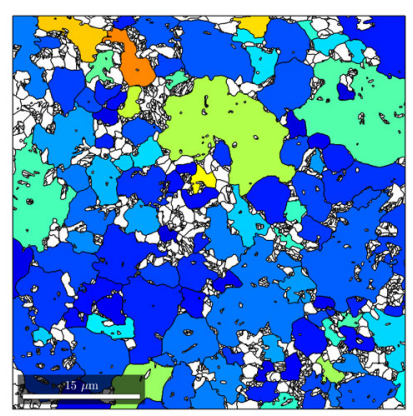

$\varepsilon_{11}=2.97 \%$

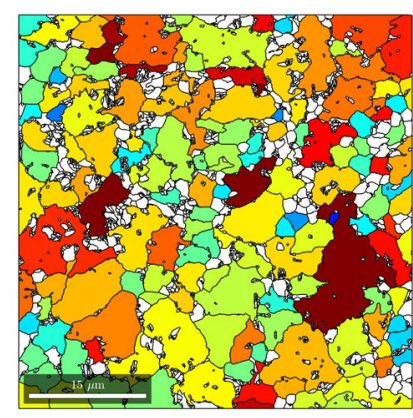

$\varepsilon_{11}=7.87 \%$

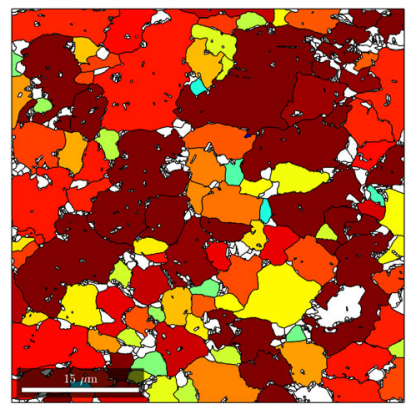

$\varepsilon_{11}=18.11 \%$

Figure 3.5: Evolution of GOS with increasing strain. White regions are martensite islands and black lines are boundaries. 
In the mostly deformed sample, it can be seen that, most of the ferrite grains have high GOS values, indicating that deformation of all the grains is highly non-uniform.

\subsubsection{Evolution of geometrically necessary dislocation density}

In this section, the evolution of GNDs is evaluated by using KAM values. We use the approach of Kubin et al. [64] to quantify the GND density. This method relates the twist angle over a unit length to the GND density as:

$$
\rho_{\mathrm{GND}}=\frac{2 \mathrm{KAM}}{u b}
$$

where $u$ is the step size in EBSD measurement and $b$ is the magnitude of the Burgers vector. In the calculations, we used the first order neighbors around a material point. There are other methods presented in literature for estimating $\rho_{\mathrm{GND}}$ by calculating a dislocation tensor [67]. In the study of Calcagnotto et al., the two methods were compared and it was shown that they give similar results [49].

Figure 3.6 shows the spatial distributions of GNDs in ferrite with increasing strain. In the initial condition, without any applied strain, the microstructure contains some GNDs locally at some of the grain boundaries and around martensite islands. The average GND content is calculated as $2.6 \times 10^{8} \mathrm{~mm}^{-2}$, which is in agreement with the values reported in the literature [49]. With increasing strain at $\varepsilon_{11}=2.97 \%$ for example, we start observing more GNDs around martensite islands and ferrite-ferrite grain boundaries. At higher strain values $\left(\varepsilon_{11}=6.00 \%\right)$, GNDs non-uniformly occupy the ferrite grains. At the highest strain level, around the maximum tensile strength, it is seen that GNDs form dense sub-structures in the ferrite grains and more diffuse GND presence is observed at the grain boundaries. The average GND content is increased to $9.11 \times 10^{8} \mathrm{~mm}^{-2}$.

Figure 3.7 shows the spatial GNDs in martensite for three strain levels: undeformed, $6 \%$ and $18 \%$. The general aspects of the evolution are in a similar trend to those in the ferrite phase. However, in the undeformed state, some small martensite islands already contain high amounts of GNDs. With increasing strain, as in ferrite, the GND content increases and forms heterogeneously distributed regions with high GND content.

Figure 3.8 and Figure 3.9 show the evolution of distribution of GNDs in ferrite and martensite. The two phases show different characteristics. Initially, ferrite shows a narrower Gaussian distribution. This can also be seen from the steep slope in Figure 3.9. On the other hand, since some small martensite islands initially contain GNDs, the distribution is wider and these small regions also affect the symmetry of the distribution as can be seen from cumulative distribution curves of martensite in Figure 3.9. With deformation, the GND density increases, the average values shift to the right and show wider scatter. Finally, the distribution exhibits asymmetric and bimodal characteristics. Ferrite shows more of a right-skewed distribution whereas martensite gives a left-skewed distribution. This asymmetric distribution of GNDs was also reported for single phase Fe-Si steels in the literature [51]. For both phases, 


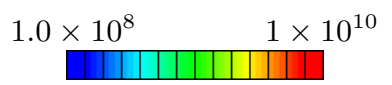

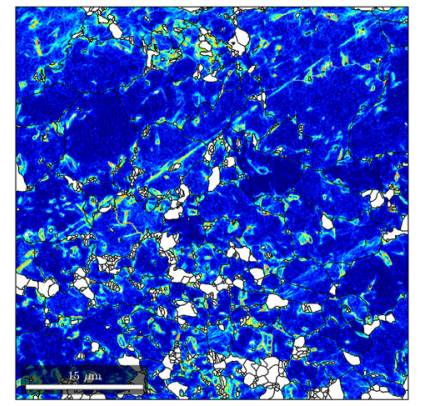

$\varepsilon_{11}=0 \%$

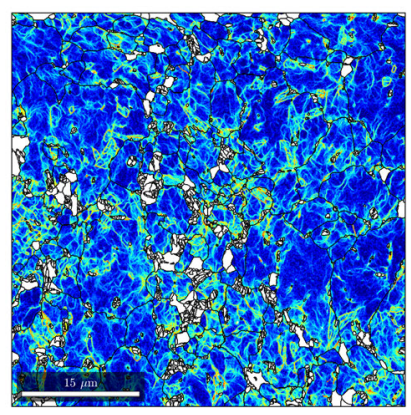

$\varepsilon_{11}=4.72 \%$

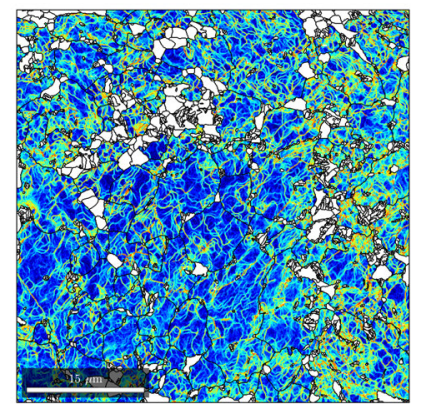

$\varepsilon_{11}=9.71 \%$

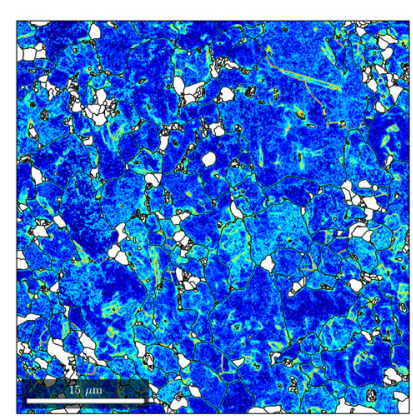

$\varepsilon_{11}=1.26 \%$

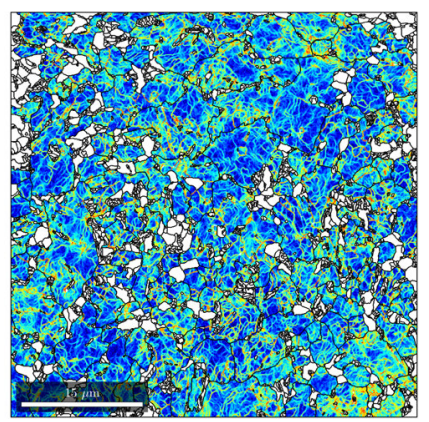

$\varepsilon_{11}=6.0 \%$

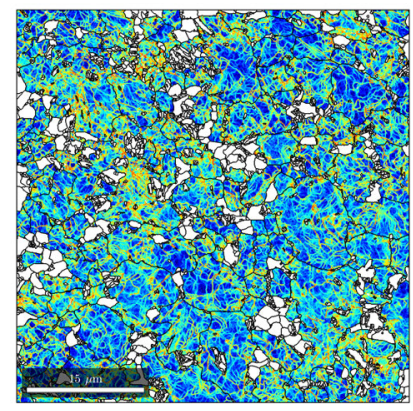

$\varepsilon_{11}=10.49 \%$

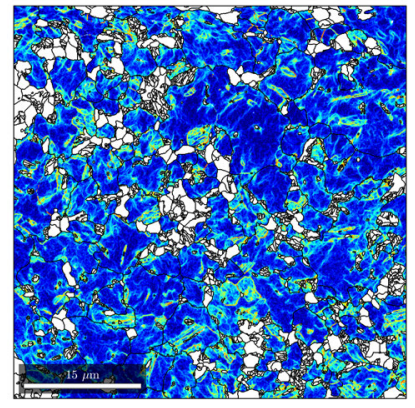

$\varepsilon_{11}=2.97 \%$

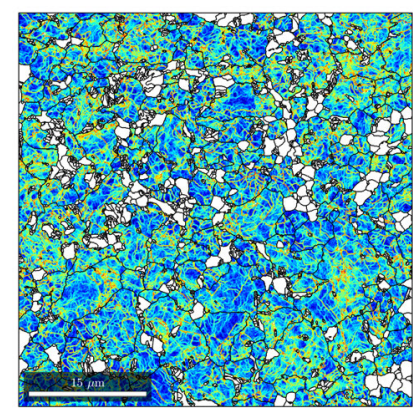

$\varepsilon_{11}=7.87 \%$

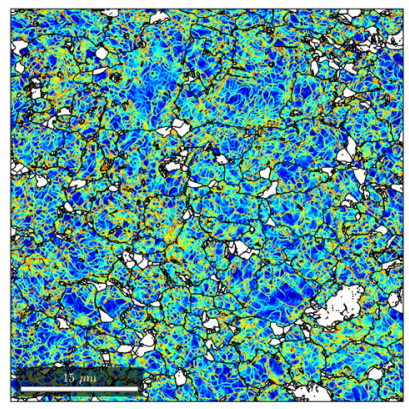

$\varepsilon_{11}=18.11 \%$

Figure 3.6: Evolution of GND density $\left(\mathrm{mm}^{-2}\right)$ in ferrite with strain. White regions are martensite islands and black lines are boundaries. 


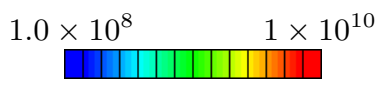

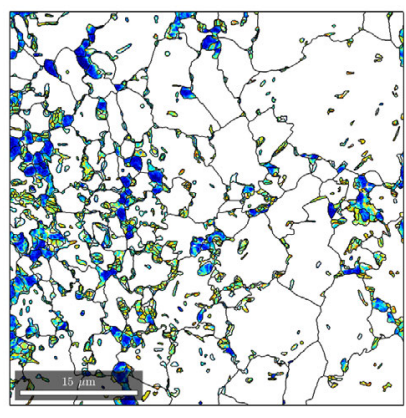

$\varepsilon_{11}=0 \%$

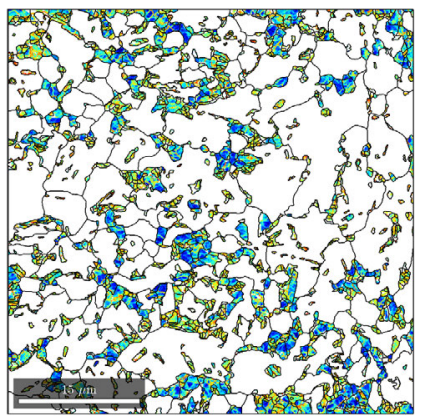

$\varepsilon_{11}=6.0 \%$

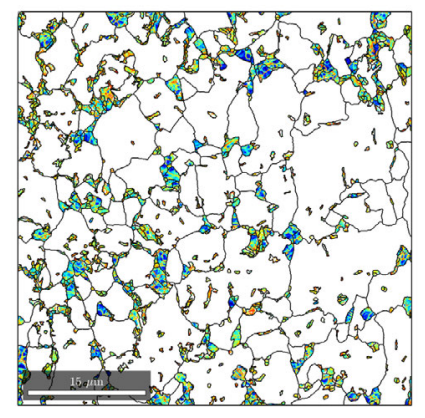

$\varepsilon_{11}=18.11 \%$

Figure 3.7: Evolution of GND density $\left(\mathrm{mm}^{-2}\right)$ in martensite with strain.

there seems to be a maximum limit in the GND density at around $4.0 \times 10^{9} \mathrm{~mm}^{-2}$, which corresponds to a $2^{\circ}$ misorientation angle, which is smaller than the KAM calculation criterion $\left(5^{\circ}\right)$.

The grain averaged GND density of ferrite and martensite grains are compared at 0.06 engineering strain in Figure 3.10. It was calculated that the region contained 127 ferrite and 1086 martensite grains in total. It is seen from the figure that for both phases smaller grain size yields a higher GND density. This is due mainly to higher ratio of the boundary and grain area in smaller grains [49]. However, for both phases we can also observe small grains with less GND content than bigger grains. This can be attributed to heterogeneity of the plastic deformation. Some grains deform more than the others in the structure depending on local conditions. This may cause a large grain to deform more than a small grain and yield a higher GND density. Thus we need to normalize the GND content with average plastic deformation of the
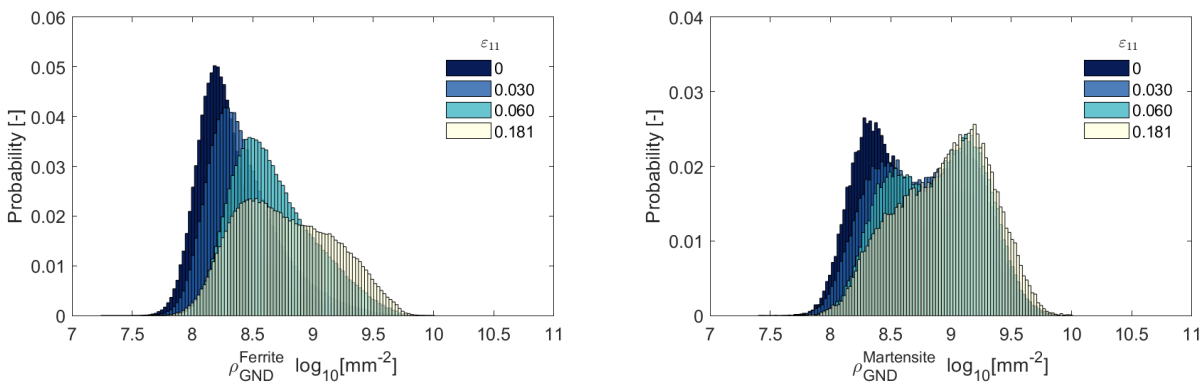

Figure 3.8: The evolution of distribution of GNDs in ferrite and martensite. 

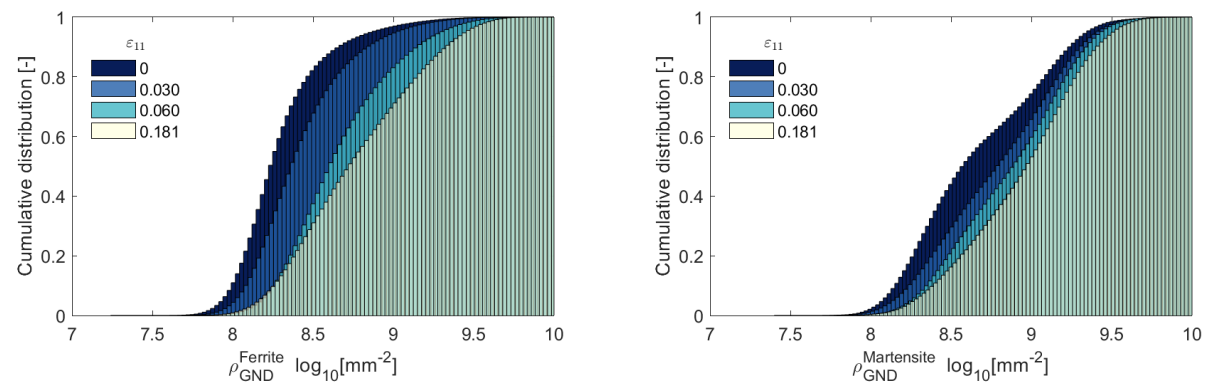

Figure 3.9: The evolution of cumulative distribution of GNDs in ferrite and martensite.
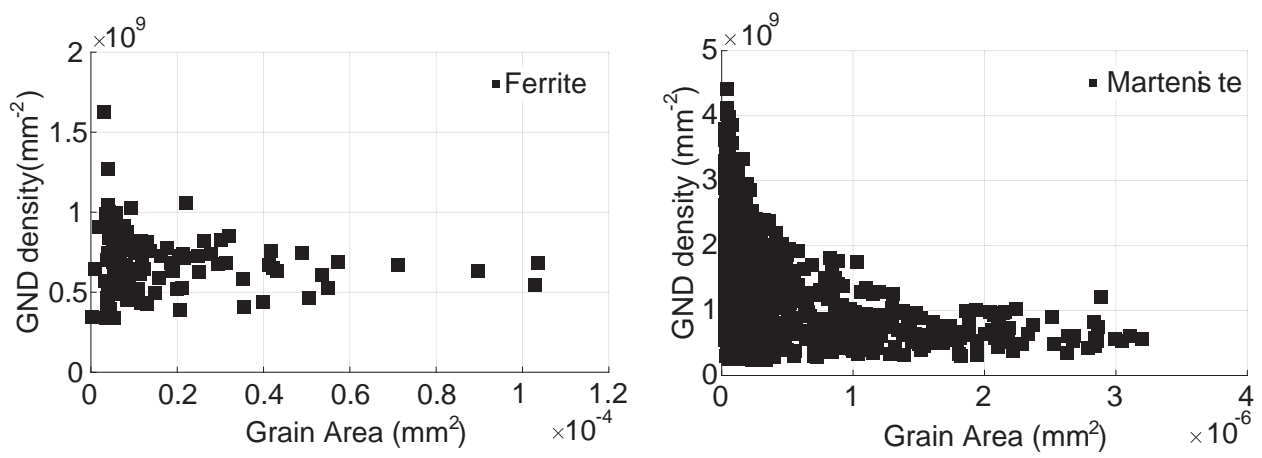

Figure 3.10: Grain averaged GND density with respect to grain size at 0.06 engineering strain.
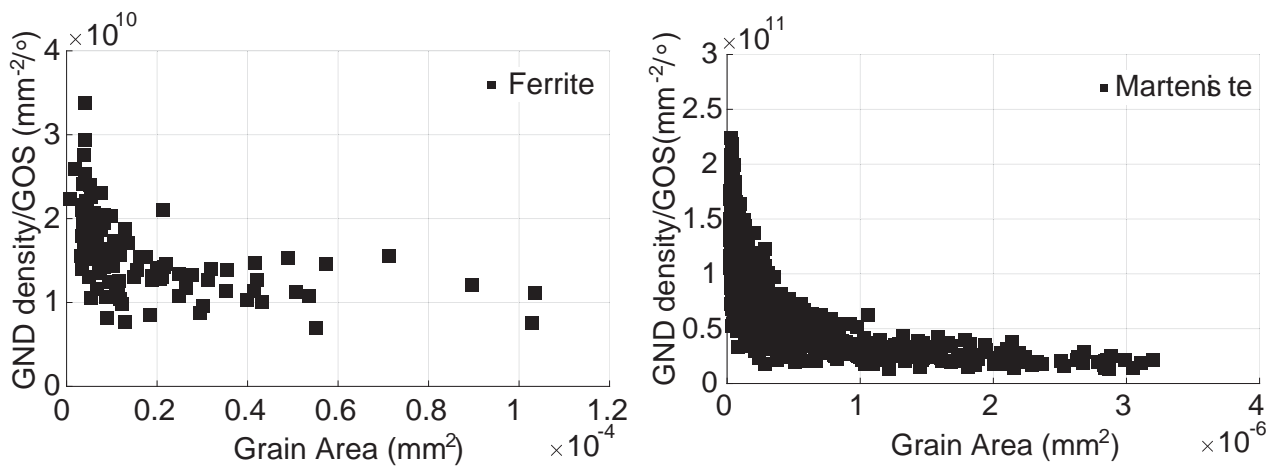

Figure 3.11: Normalized grain averaged GND density with respect to grain size at 0.06 engineering strain. 

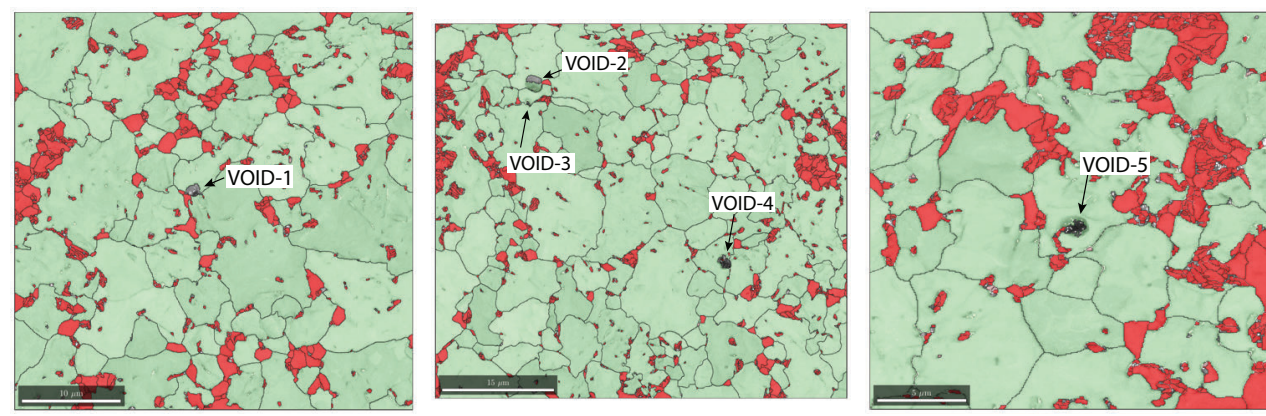

Figure 3.12: Phase identification superimposed to band contrast images. Voids are shown with arrows. Green colored phase is ferrite, pink color indicates martensite islands and black lines show grain boundaries.

grain in order to obtain more reliable results. Here, for normalizing the GND density, we employ GOS values of the grains, which is sometimes expressed as a function of plastic deformation of a grain [56]. Figure 3.11 shows the normalized GND density with respect to grain size. It is clearly seen that smaller grains yield higher GNDs.

\subsubsection{Plastic heterogeneity around voids}

Here, grain reference orientation deviation (GROD), grain orientation spread (GOS) and geometrically necessary dislocation density around voids are investigated. We have conducted the investigation on the sample deformed to 0.06 engineering strain. The measurements were conducted with the same settings (step size, acceleration voltage, sample tilt, CCD binning, etc...) but with different magnification and the measurement areas depend on the size of voids.

Figure 3.12 shows the location of the voids. The green color indicates ferrite and the pink color shows the martensite islands. The void locations were confirmed also by the secondary electron images. The first observation is that two of the voids are located at the ferrite-martensite interface (void-1 and void-5), two voids are at the ferrite grain boundary (void-3 and void-4) and one void (void-2) is in the ferrite grain but it is very close to a martensite island.

Figure 3.13 shows the GROD and GOS values around the voids. In the figure, the voids are marked with white circles and martensite phase is indicated with pink color. GROD maps do not show necessarily high values at the locations where voids are present at the grains. There are regions in the same grains usually with higher GROD values. Moreover, the GOS maps also show that the grains which have voids, are not the ones with highest deformation heterogeneity. These two observations indicate that void nucleation is affected by local conditions in the grains. It does not necessarily take place in the highly non-uniform location (GROD) of a highly nonuniform grain (GOS). Moreover, since high GOS and GROD values are indicators of high plastic strain, we can also say that void nucleation is not solely a plastic strain driven event. 


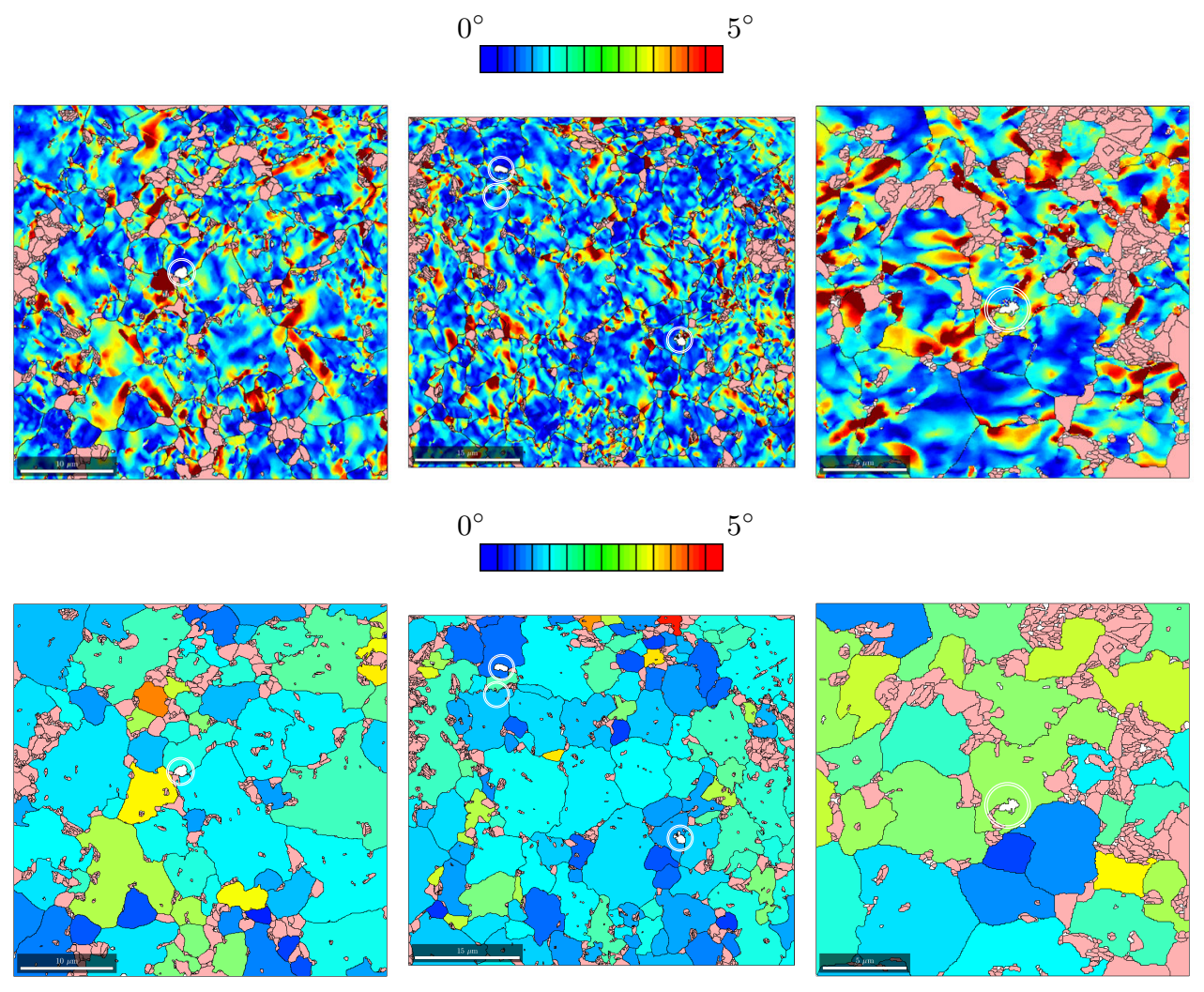

Figure 3.13: GROD (top) and GOS (bottom) around the voids. Pink colored islands indicate the martensites. Voids are circled with white colored double line.

Finally, we will look at the $\rho_{\text {GND }}$ distribution in the microstructures. Figure 3.14 shows the overall structure, whereas Figure 3.15 shows only the surrounding of the voids. From these figures, it is seen that there is usually high GND density surrounding (at least one side) the voids. However, in the structures there are also locations without voids where higher GND content is present. It was not possible to conclude whether GND content around the voids increased before or after the nucleation of the voids. 

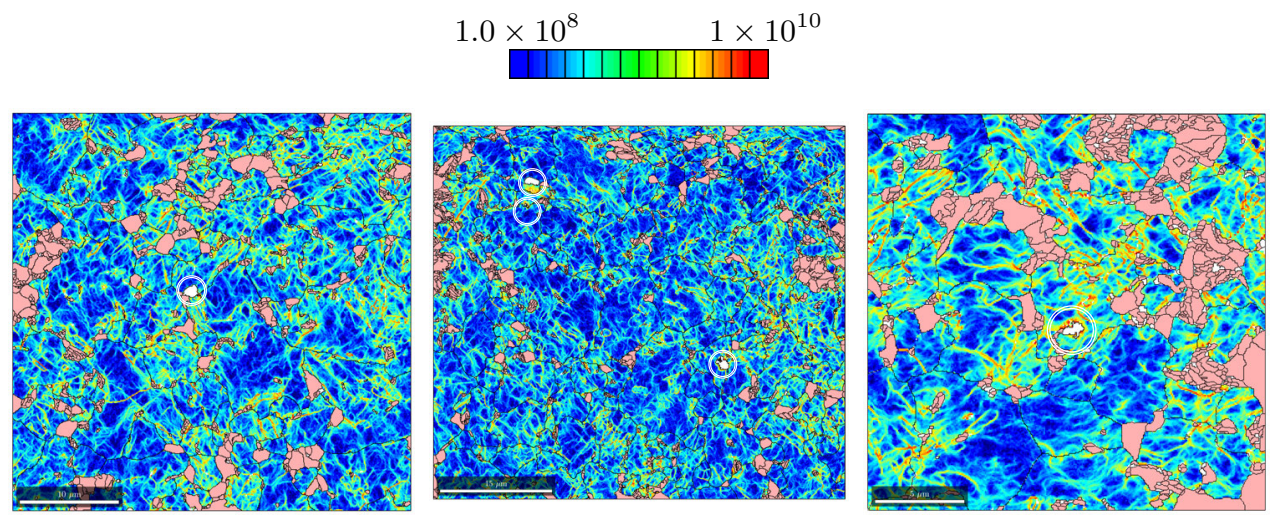

Figure 3.14: GND density $\left(\mathrm{mm}^{-2}\right)$ around the voids. Pink colored islands indicate the martensites. Voids are circled with a white double line.

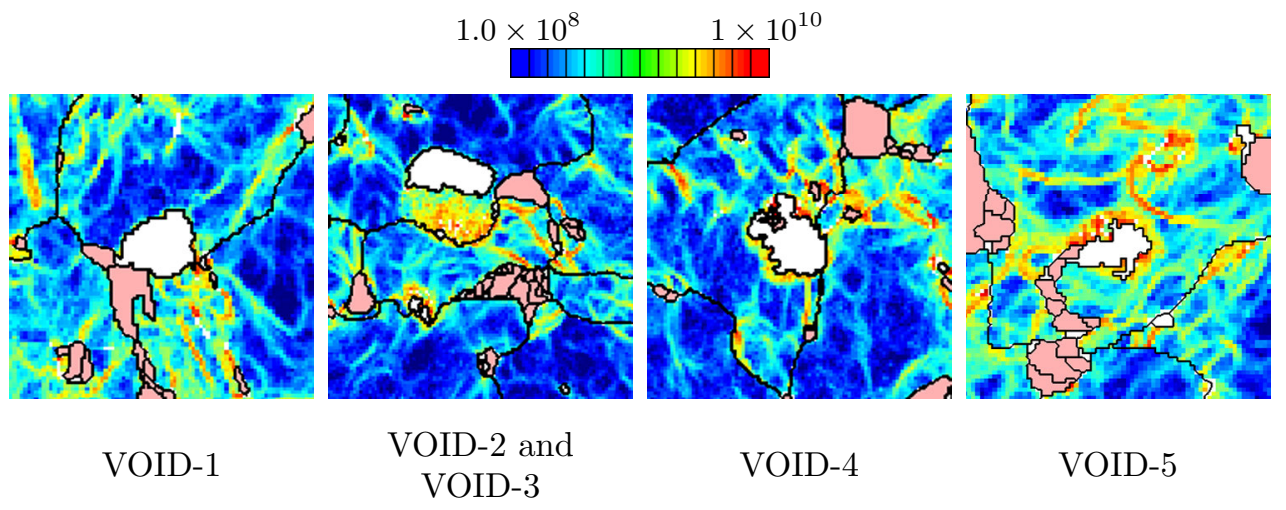

Figure 3.15: Cropped images showing GND density $\left(\mathrm{mm}^{-2}\right)$ in the ferrite surrounding the voids. Pink colored islands indicate the martensite. 


\subsection{Conclusions}

In, this chapter, we have investigated the evolution of microstructural orientations for a DP600 steel with increasing strain. Plastic deformation in DP steels is highly heterogeneous due to the combination of lattice orientations and a nonuniform distribution of mechanically very diverse phases. Application of plastic deformation causes grain orientations to evolve in various manners. Grain reference orientation deviation (GROD) and grain orientation spread (GOS) are two orientation relationships that can be used to quantify plastic heterogeneity in the grains. Kernel average misorientation (KAM) is used to quantify the GND density.

From the investigations, it is found that:

- Initially there are small orientation variations in the ferrite grains due to production conditions.

- Initially ferrite has an average of $2.6 \times 10^{8} \mathrm{~mm}^{-2}$ GND density.

- With increasing deformation, non-homogeneous deformation of the grains increase GROD, GOS and $\rho_{\mathrm{GND}}$.

- There seems to be a limit to the maximum $\rho_{\mathrm{GND}}$ of $4.0 \times 10^{9} \mathrm{~mm}^{-2}$ that can be accommodated within grains.

- The average GND density increases with deformation for both ferrite and martensite, causing a right-skewed distribution for ferrite and a left-skewed distribution for martensite.

- Void nucleation is effected by local conditions. High nonuniformity in grain orientations does not necessarily make a nucleation site. This suggests that void nucleation is not a purely plastic strain related phenomenon.

- High GND density was observed around the voids. 



\section{Damage mechanisms in DP600 steel}

This chapter investigates active damage mechanisms (martensite cracking, void nucleation at the ferrite-martensite interface) and evolution of damage in a commercial DP600 steel. Interrupted tensile tests were conducted in both rolling (RD) and transverse directions (TD). Subsequently, damage mechanisms and void evolution were characterized by cross-sectional SEM micrographs. The results reveal that, in both RD and TD, damage is caused by three different damage mechanisms. Namely, void formation due to inclusions, cracking of martensite islands and decohesion between ferrite and martensite. From these damage mechanisms, void formation due to large inclusions occurs in the early stages of deformation, whereas the other two are both active throughout the complete stretching. The most commonly observed damage mechanism was martensite cracking and this seems to be the primary reason of failure. In addition, void evolution studies clearly show that both the damaged area and the number of voids increase more rapidly in RD than TD. Furthermore, in both directions, damage concentrates at the mid plane of the specimens, leading to an inhomogeneous distribution of voids in the thickness direction.

\subsection{Introduction}

In dual phase steels, it is thought that damage initiates due to local strain and stress concentrations during deformation. These concentrations occur in specific locations: within martensite islands, between ferrite and martensite phases, around inclusions and at ferrite grain boundaries. In the literature, there is no certain guideline on the order or importance of different damage mechanisms. This is mainly due to the

Reproduced from:

EE Aşık, ES Perdahcıoğlu, and AH van den Boogaard.

Microscopic investigation of damage mechanisms and anisotropic evolution of damage in dp600.

Materials Science and Engineering: A, 739:348-356, 2019. 
complex interaction between phases as well as variation in the mechanical properties of ferrite and martensite $[19,21,68-70]$. On the other hand, it is clear that damage evolution in dual phase steels occurs in three stages; void initiation, growth and coalescence [71-73].

Experimental research on damage mechanisms can be classified into two main groups: in-situ and post-mortem analyses. In-situ methods have the advantage of correlating sequential images and gathering history dependent information albeit only from the surface. Developments in X-ray micro-tomography may overcome this restriction of in-situ methods by providing 3D information [74]. Post-mortem methods, on the other hand, give a better understanding of what has happened under the surface. In the work of Taşan et al. [75], in-situ analyzes combined with scanning electron microscopy (SEM) were used to investigate void nucleation in the thick central martensite band and local strain distribution between ferrite and martensite. It was shown that void nucleation sites and high strain bands were coinciding. Maire et al. [74] used X-ray micro-tomography and quantified void initiation and evolution in a volume under increasing strain. This study gives valuable insight on the distribution of voids along different directions (rolling, transverse).

Alternatively, post-mortem analyzes are frequently used for damage characterization by many researchers [18, 76-79]. Avramovic-Cingara et al. [18], investigated two different DP600 steels. They observed martensite cracking, and ferrite-martensite decohesion. In addition, they commented qualitatively on the high number of voids at the center martensite band. On the other hand, Kadkhodapour et al. [19] studied damage mechanisms in a commercial DP800 steel and they observed mainly ferriteferrite interface damage. Lai et al. [79] worked on dual phase steels with different martensite fractions. They found that the martensite fraction had an effect on the dominating damage mechanism. At high martensite fractions cracking of martensite was the main mechanism, whereas at low martensite fractions interface decohesion was more important.

In this chapter, we use post-mortem analyzes and scanning electron microscopy to investigate active damage mechanisms and evolution of voids in a DP600 sheet. For this purpose, interrupted tensile tests were conducted both in rolling (RD) and transverse directions (TD). Damage mechanisms were characterized in terms of void nucleation sites, while damage evolution was quantified in terms of $\%$ damaged area, number of damage incidents, average nearest neighbor distance, and void distribution. In order to differentiate between void nucleation and growth characteristics, voids were categorized depending on their size. Finally, similarities and differences were compared for RD and TD.

\subsection{Experimental details}

\subsubsection{General characteristics of DP600 sheet}

The material used in this study is a commercial cold-rolled and annealed DP600 steel sheet with a thickness of $1.20 \mathrm{~mm}$. Composition of the alloying elements is given 

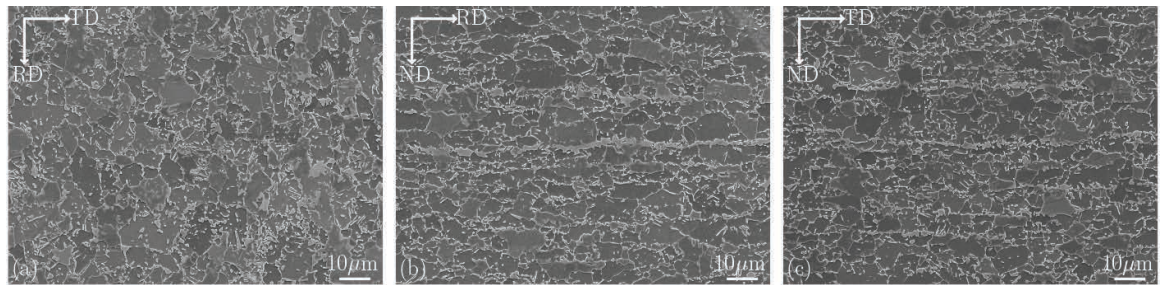

Figure 4.1: Initial microstructure of the DP600 sheets looking from (a) normal, (b) transverse and (c) rolling directions. Dark gray matrix is ferrite and the lighter colored islands are martensite.

in Table 4.1. The material has a dual phase microstructure, which consists of ferrite matrix and martensite islands. Additionally, negligible amount of (less than $0.1 \mathrm{vol} \%$ ) cementite and retained austenite are present. The initial microstructure of the steel sheet is shown in Figure 4.1. the martensite phase exhibits a random distribution when observed from the sheet normal, Figure 4.1(a). However, micrographs taken from rolling and transverse directions clearly show the banded microstructure that is present in DP600 sheet, Figure 4.1(b and c). This type of microstructural inhomogeneity is very common in steel sheets. Banded distribution of the phases is attributed to segregation of substitutional alloying elements during solidification at the interdentritic spaces. Subsequent deformation causes elongated and parallel bands of high-solute and low-solute rich regions, which later on influence the transformation temperatures $[80,81]$.

\subsubsection{Interrupted tensile tests}

Interrupted tensile test samples had a standard geometry with a gauge length equal to $75 \mathrm{~mm}$ and a width equal to $12.5 \mathrm{~mm}$ as described in ASTM-E8 [82]. Additionally, the thickness of the samples was equal to the sheet thickness, $1.2 \mathrm{~mm}$. Samples were prepared along the rolling (RD) and transverse (TD) directions from the DP600 sheet. Interrupted tensile tests were conducted at room temperature with a $100 \mathrm{kN}$ capacity screw driven electromechanical test system (Zwick Testing Machines Ltd, UK). During the tests, a cross-head speed of $5 \mathrm{~mm} / \mathrm{min}$, which corresponds to an initial strain rate equal to $0.001 \mathrm{~s}^{-1}$, was kept constant. The average longitudinal strain was recorded with a non-contact laser speckle extensometer (Messphysik Materials Testing GmbH, Austria) over a relatively large gauge length of $40 \mathrm{~mm}$. This length was intentionally selected in order to minimize the possibility of necking close to the strain measurement

Table 4.1: Chemical compositions (wt\%) of the alloying elements in DP600 sheet.

\begin{tabular}{ccccc}
\hline $\mathrm{C}$ & $\mathrm{Cr}$ & $\mathrm{Mn}$ & $\mathrm{Si}$ & $\mathrm{P}, \mathrm{S}$ \\
\hline 0.09 & 0.5 & 1.9 & 0.06 & Trace \\
\hline
\end{tabular}


Table 4.2: Mechanical Properties of the DP600 sheet in rolling (RD) and transverse directions (TD).

\begin{tabular}{cccc}
\hline & $\begin{array}{c}\text { Ultimate } \\
(\mathrm{MPa})\end{array}$ & $\begin{array}{c}\text { Uniform } \\
\text { Tensile Strain } \\
(\%)\end{array}$ \\
\hline $\mathrm{RD}$ & $400 \pm 9$ & $660 \pm 6$ & $18 \pm 0.15$ \\
$\mathrm{TD}$ & $412 \pm 6$ & $659 \pm 2$ & $17 \pm 0.36$ \\
\hline
\end{tabular}

points. Three reference tensile tests were conducted, in both directions, until failure during which the strain field was determined by a digital image correlation system (Aramis, GOM GmbH, Germany). In the calculations, a facet size of $15 \times 15$ px was selected and the distance between two facets was 13 px.

Table 4.2 shows some of the crucial mechanical properties of the sheet in RD and TD. The values are calculated by averaging five measurements found from the interrupted tensile tests in which the interruption point is further than the necking strain. It can be seen that the standard deviation of the properties is rather small. For that reason, a single sample was tested for each interruption point, which makes 14 samples in total. Seven interruption points were chosen to stop the tests. Two of these were before the necking point, i.e. between 10-18\% engineering strain. For the remaining five interruption points, different percentages of load drop from the UTS point, were used. These levels were chosen as $1 \%, 3.5 \%, 5 \%, 7.5 \%$, and $10 \%$, which cover a wide range to observe the evolution of damage. Figure 4.2 shows the engineering stress-strain curves for the steel sheet. For clarity, the interruption points are shown with markers on the curves. It can be seen that until necking occurs, the deformation response in both directions are very similar; however, failure occurs faster in transverse direction.

In the literature, this type of study is mostly carried out on a cross-section of a failed specimen where the evolution is later correlated from the thickness change of the tensile test sample $[18,79,83]$. However, this approach has two basic disadvantages. Firstly, strain localization at the neck reaches values of around 50-60\% before fracture and it decreases with a very high gradient back to values of around $15 \%$. As a result, the possible measurement areas are confined to very small regions. This can be seen from the results of digital image correlation (DIC) measurement in Figure 4.3 which shows true longitudinal strain at the center line (across width) of samples just before failure as a function of distance from the localized region (neck). Although the local longitudinal strain in the TD sample was higher than in the RD sample the opposite is seen on the average strain measurement. Secondly, these studies associate relative thickness reduction with the longitudinal strain, which is a questionable assumption. This is due to the fact that in order to use thickness reduction, one measures the average thickness strain which, due to the strain in the width direction, might evolve differently. 

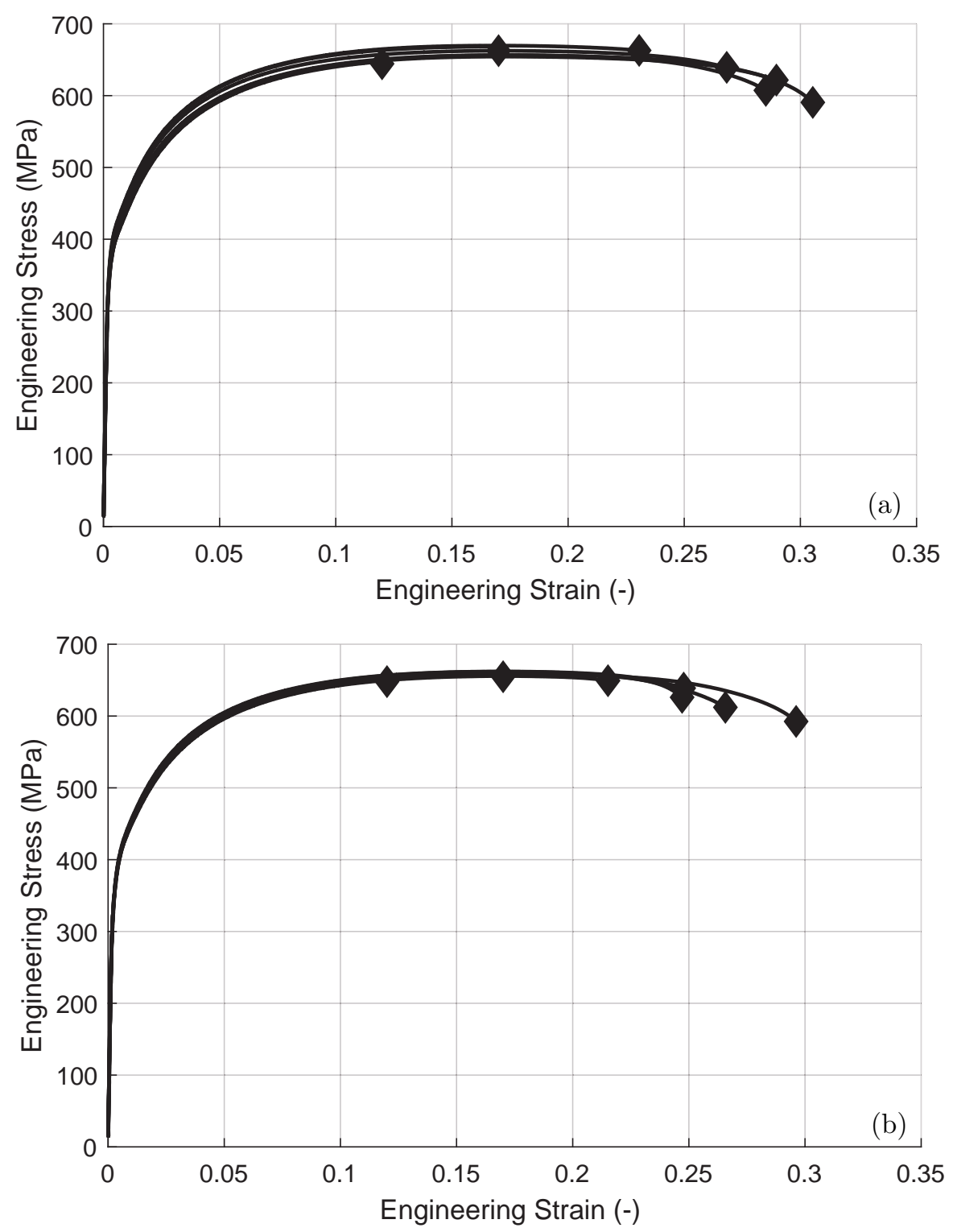

Figure 4.2: Engineering stress strain response of DP600 sheet loaded along a) rolling and b)transverse directions. Interruption points showed with round markers.

Figure 4.4 compares two different methods of calculating longitudinal strain at the neck. The strain denoted on the $\mathrm{x}$-axis is approximated by matching the stress level of each specimen at the interruption point to that of three reference DIC measurements. This leads to an approximation of the strain value with a level of uncertainty. However, it can be seen that apart from one of the points, the deviation 


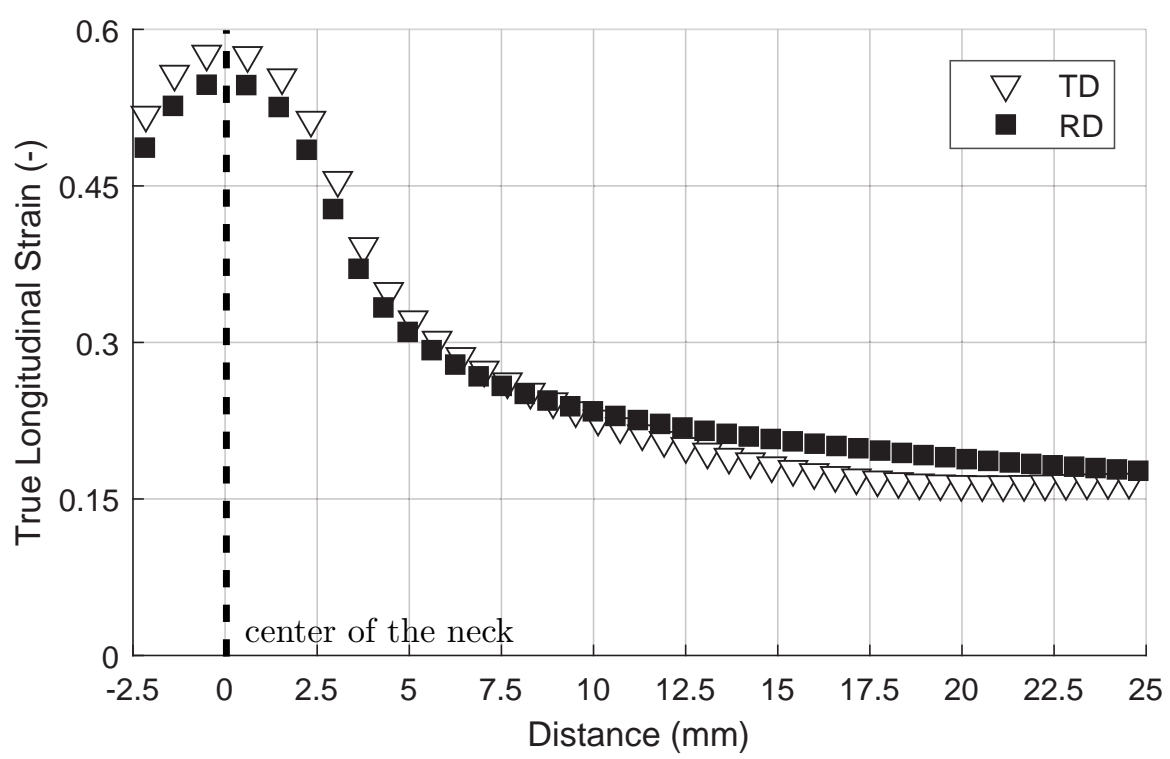

Figure 4.3: True longitudinal strain distribution along reference sample. Dashed line indicates position of localization.

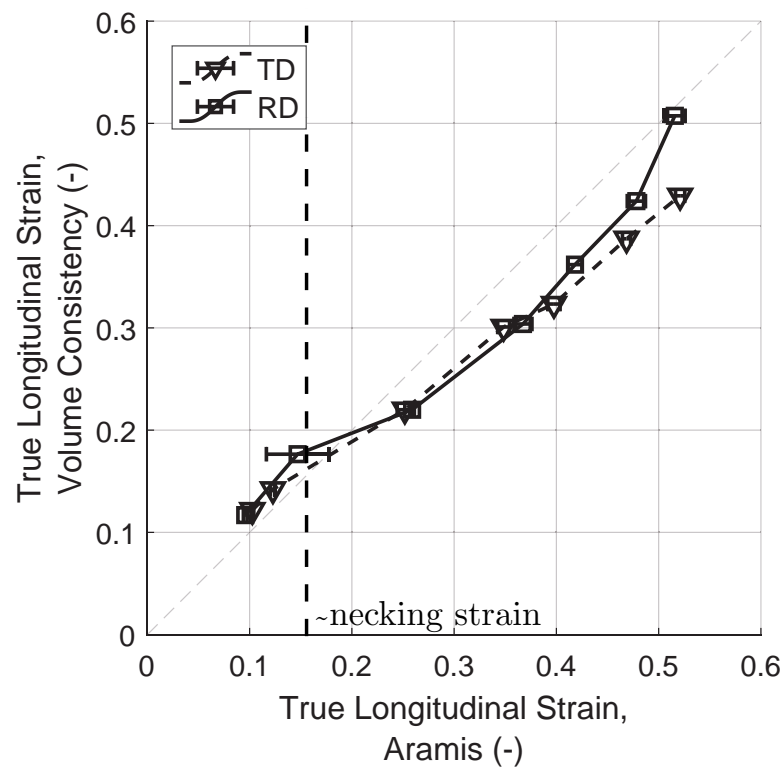

Figure 4.4: Comparison of calculated strain from values with respect to the measured strain by Aramis. Vertical dashed line shows necking strain.

is small. The uncertainty occurs due to the very small slope of the load-displacement curve. The y-axis, on the other hand, is estimated by assuming no volume change 


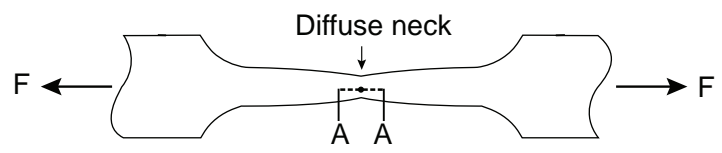

Figure 4.5: Schematic top-view of the sample after interrupted tensile test. The section denoted by A-A shows the position and the orientation of the metallographic samples.

during deformation and is calculated from the width of the thinnest part and from the thickness of the imaging area of the actual specimens. The figure shows that after necking the calculated strain underestimates the values inside the neck. This is due mainly to the effect of strain concentration at the center of the samples. Based on this observation, the Aramis measurement is deemed to be more reliable and therefore used as the main strain reporting measure in this work.

\subsubsection{Metallographic investigations}

A field emission scanning electron microscope (Jeol-SEM 7200F Ltd, Japan) was used for imaging microstructures. The images were recorded with a resolution of $2560 \times 2048$ pixels . In addition, the microscope was equipped with 2 EDX detectors (Oxford Instruments plc, UK) for elemental analysis.

Metallographic cross-sections, along the loading direction, were prepared from the localized part of the interrupted tensile test samples. For the samples without localization, a random central section was used. The section is denoted A-A in Figure 4.5 , which shows a schematic representation of the region of interest (ROI). Samples were cut by an abrasive cutter and subsequently, embedded in Bakelite. Afterwards, metallographic samples were ground down to 4000 grit $\mathrm{SiC}$ paper followed by polishing in the range of 3 to $0.25 \mu \mathrm{m}$ diamond suspensions. Specimens were immersed into $4 \%$ Nital solution for seven seconds in order to have sufficient contrast between ferrite and martensite.

\subsubsection{Image processing}

Void evolution was quantified by using the polished cross-sectional micrographs. The images were captured at 250x magnification, which corresponds to an area equal to $0.18 \mathrm{~mm}^{2}$. This magnification scale satisfies a resolution high enough to identify voids as well as an area large enough to observe general characteristics of void properties such as size, number, and distribution. In order to quantify the voids, a 4-step MATLAB $^{\circledR}$ algorithm was developed, as shown in Figure 4.6.

The algorithm is based on binarizing the image after identifying void edges. Firstly, the background noise was removed by subtracting the original image from the blurred image. Image blurring was applied using a large (100 px) Gaussian filter. Secondly, a compound edge image was calculated by combining two different edge detection 

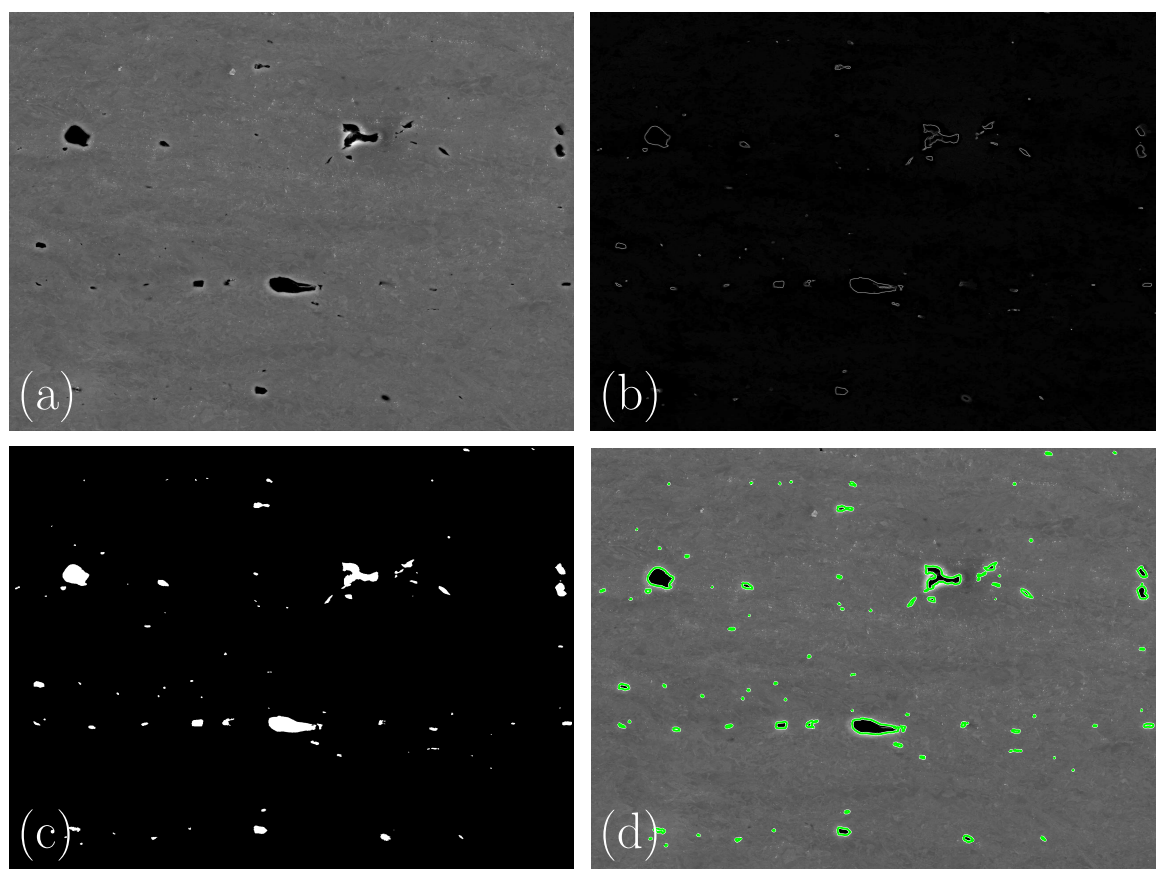

Figure 4.6: Automated image processing. (a) original image, (b) edges image, (c) black and white image and (d) analyzed voids highlighted in green. 
methods. The first edge image was calculated by using the gradient of the pixel values in $\mathrm{x}$ and $\mathrm{y}$ directions. The second one was obtained by using Kirsch compass kernels in all eight directions [84]. The combination of these two creates an image that is sharper and has well defined edges. In the third step, the edge image was transformed into a binary, black and white, representation. Thanks to the high contrast obtained by the edge detection step, a standard automated threshold detection is sufficient for this transformation. It is important here to note that by using the aforementioned steps, one can fully automatize image processing and minimize human interaction which will result in more standardized and unbiased results. Finally, edges of voids were indexed by using the bwboundaries function that is found in the standard image processing toolbox of MATLAB ${ }^{\circledR}$ by setting a critical size of 4 pixels $(0.14 \mu \mathrm{m})$. Smaller features than the critical size were not considered as a void.

\subsection{Results and discussion}

\subsubsection{Damage mechanisms}

This section approaches damage in a qualitative manner. The main aim is to understand and characterize active damage mechanisms in DP steels leading to failure. In the literature, three damage mechanisms, i.e. void nucleation sites, are commonly reported for ferritic-martensitic dual phase steels $[18,19,21,68,79,85]$. These mechanisms are classified according to their relationship with the surroundings. In this section, observed damage mechanisms and their general characteristics are discussed.

The first of the observed damage mechanisms is void formation due to inclusions. In steel sheets, inclusions are inevitable and any inclusions present cause early void formation. Despite the fact that with modern production techniques, the number of inclusions is limited, completely avoiding them is still not possible. Figure 4.7 shows a rare event where Figure 4.7(a) is the secondary electron image, with the arrow indicating loading direction and the white solid box and two white circles showing damage due to inclusions. This shows that during the early stages of deformation inclusions can cause void formation by decohesion. In this case also MnS inclusions crack due to their rod-like shape. Further information on this type of damage can be found in $[86,87]$.

The second mechanism for void formation is martensite cracking which was the most common mechanism observed in this study. Figure 4.8 shows some example cases both for samples along TD and RD. Initially, a crack nucleates at one side of the martensite as in Figure 4.8(a and b). Subsequent deformation propagates the crack perpendicular to loading direction, Figure 4.8(c). In the end, crack reaches the other end of martensite island and voids starts growing in the loading direction due to deformation of surrounding ferrite, Figure 4.8(d). This process results in elongated voids with the thickness of the martensite island, that it formed to.

The last mechanism for void formation is decohesion between martensite and ferrite. In Figure 4.9 demonstrative examples of damage occurring at the interface are 

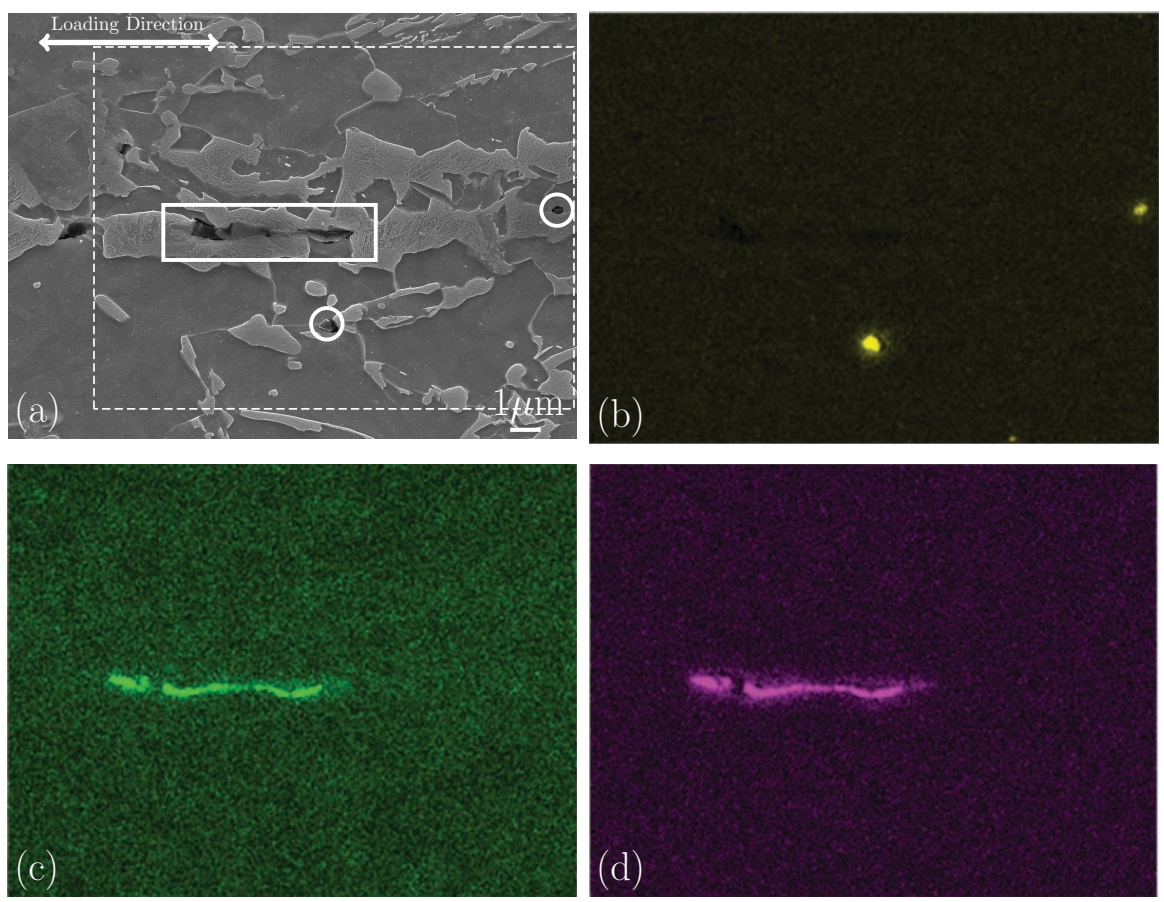

Figure 4.7: Voids nucleated due to inclusions. (a) Secondary electron image showing; the scanning area for elemental distributions as the white dashed box and with white solid box and white circles the Al-based and MnS inclusions. EDX map showing the elemental distributions for (b) Al ,(c) Mn and (d) S. Loading direction is horizontal. 
presented. A general characteristic of interface damage is that void growth occurs in the direction of plastic flow. However, in the early stages of void growth, grain boundaries and orientation of ferrite play an important role. As can be seen from Figure 4.9(c), a void does not grow elliptically in the loading direction, instead, it has sharp corners in the ferrite grain. Moreover, it does not grow equally into the neighboring ferrite grain.

As deformation proceeds, damage incident density increases. Consequently, voids formed by different mechanisms can be observed together in a small area. Figure 4.10 shows three voids formed by martensite cracking and ferrite-martensite decohesion mechanisms. As a common observation, these voids also exhibit a banded structure as observed in the distribution of martensite. Later on, these aligned voids may coalesce easily by the 'necklace' mechanism $[10,88]$.

To summarize, for the DP600 steel used in the present study, the main mechanism leading to failure is coalescence of voids that are caused by martensite cracking. During deformation, martensite cracks form weak spots and form voids. This leads to a decrease in the load-carrying capability of the microstructure, causing extensive localized deformation of ferrite surrounding the voids. In the meantime, voids form at the interfaces due to the mismatch between plastic deformation of martensite and ferrite. As deformation continues, more and more voids form by these two mechanisms and ferrite grains keep deforming till formed voids coalesce, resulting in failure. In addition, void nucleation inside ferrite grains are reported in literature, but they were not observed in the current experiments.

\subsubsection{Damage evolution}

In this section, a quantitative approach is followed in order to understand the damage evolution during straining of DP600 sheet. The main focus points are the change in void area percentage, the number of voids and the average nearest neighbor distance.

Figure 4.11 shows the increase in total damaged area with respect to increasing strain as observed in a specific area of the cross-section. As a first observation, it is noted that void formation occurs before macroscopic localization, i.e. diffuse necking starts, which takes place at approximately $15 \%$ strain. Secondly, damage evolution occurs more slowly and almost linearly up to a certain level of strain. This level is around $42 \%$ for RD and significantly lower, at around 26\%, for TD. Further deformation results in an exponential increase of void area.

Figure 4.12 shows the evolution in terms of the number of voids. It is observed that the number of damage instances evolve similarly to the damaged area fraction. However, the level of strain at which the number of voids starts increasing exponentially is slightly at lower strain valuesthan at the values in areal evolution.

In order to distinguish void nucleation and growth, voids are categorized depending on their areal size, in Figure 4.13 and 4.14. Damage incidents smaller than $0.5 \mu^{2}$ are classified as freshly nucleated voids, whereas bigger incidents are classified as grown voids. The characteristic behavior is that with increasing strain, both number of voids which are bigger (grown) and smaller (freshly nucleated) than the critical 

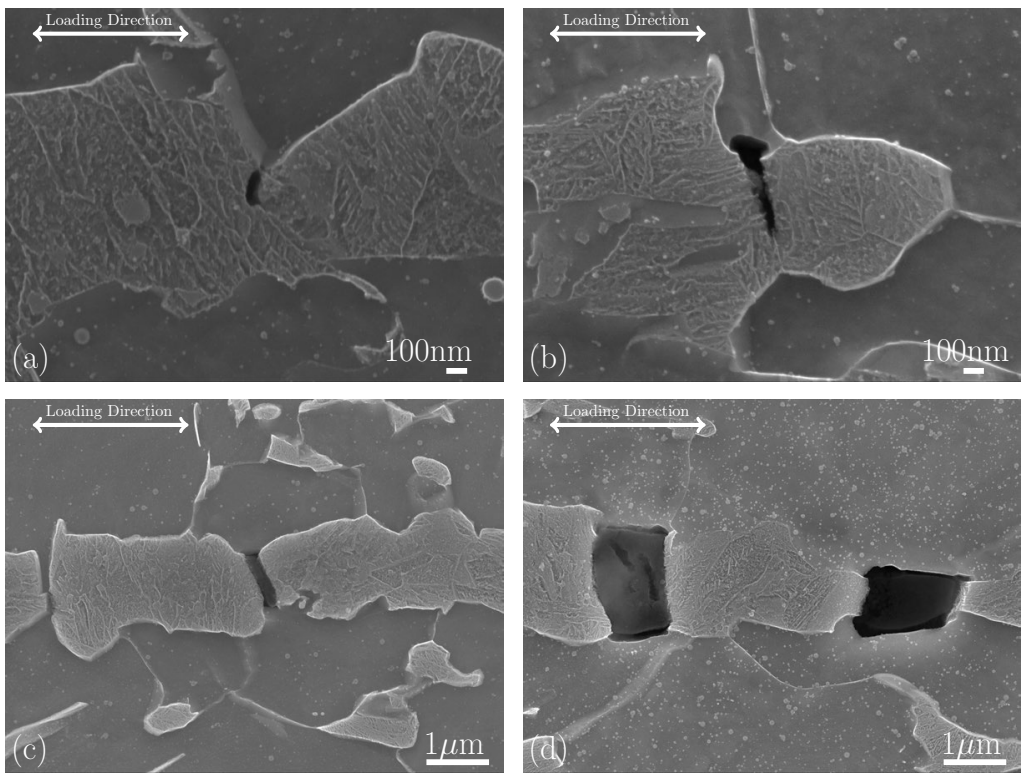

Figure 4.8: Voids formed by cracking of martensite. (a), (b) sample stretched in TD and (c), (d) sample stretched in RD. Loading direction is horizontal.
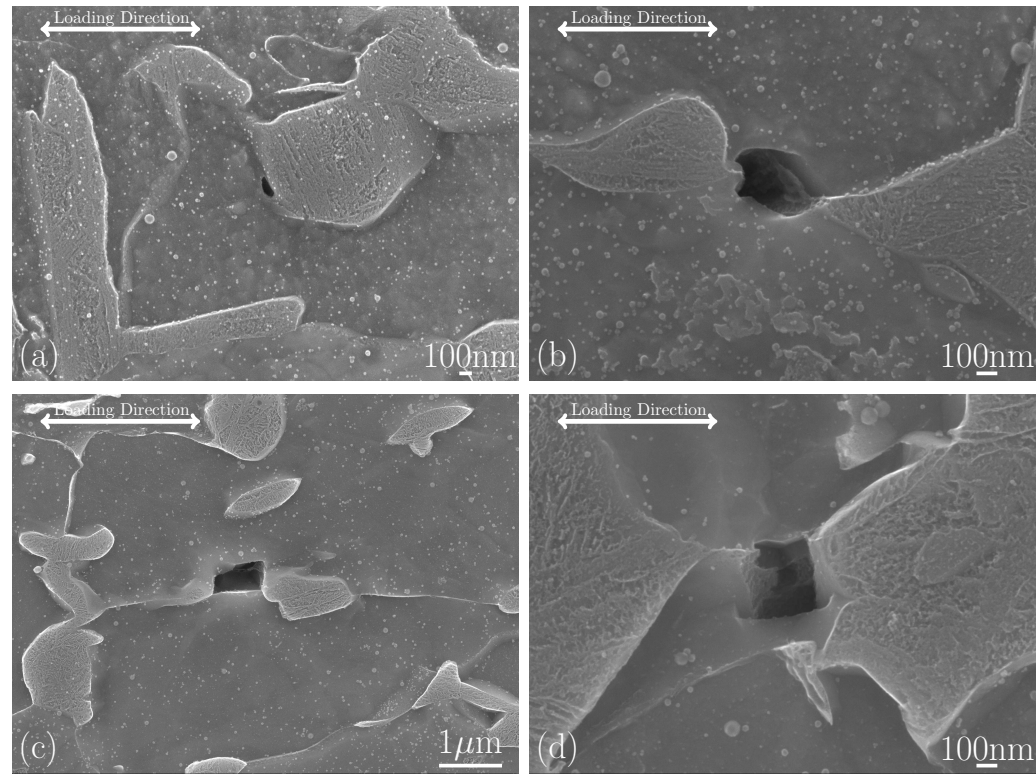

Figure 4.9: Voids formed by interface decohesion between ferrite and martensite. (a), (b) sample stretched in TD and (c), (d) sample stretched in RD. Loading direction is horizontal. 


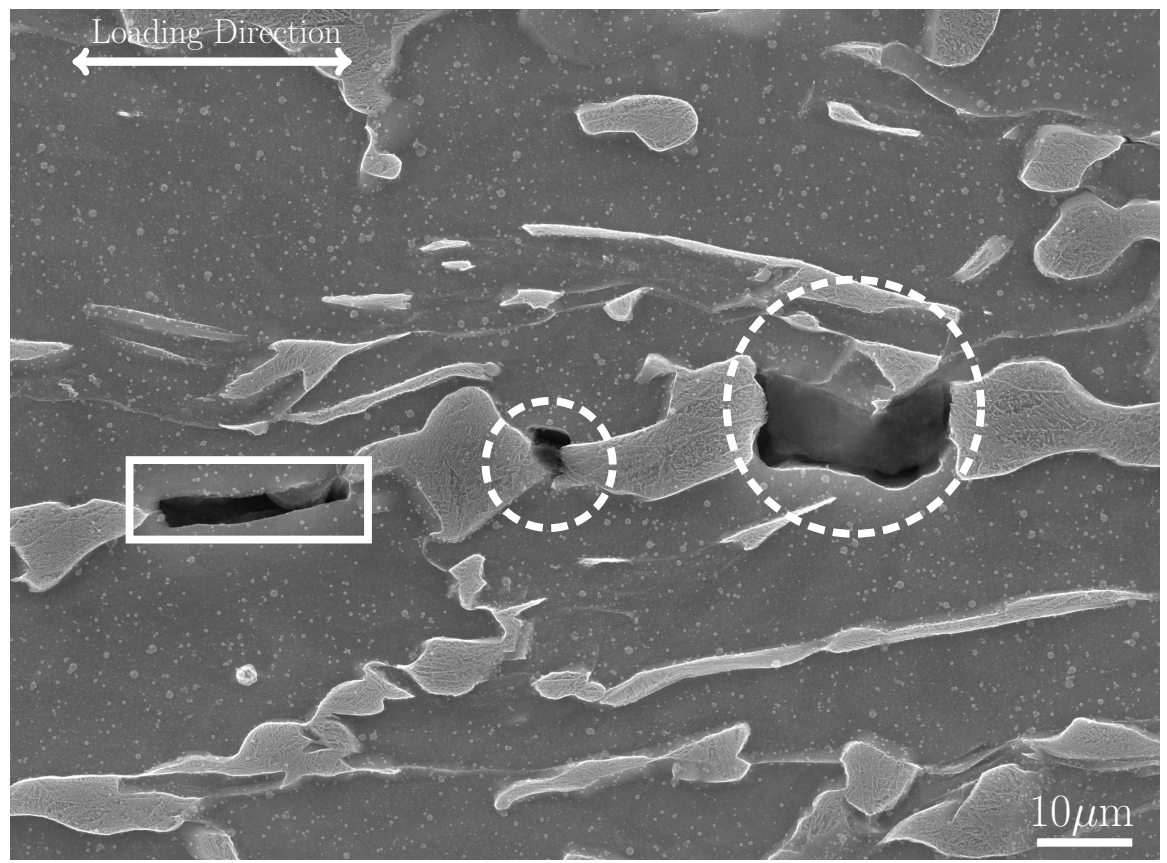

Figure 4.10: Aligned voids along loading direction. White solid square shows and interface damage between martensite and ferrite. White dashed circles show voids formed by cracking of martensite. Loading direction is horizontal.

size increase. This suggests that during deformation both void nucleation and void growth take place. It can also be seen that the number of big voids increase more quickly than the number of small voids. In the samples loaded along the TD, at a strain range between 0.25 to $0.35 \%$, a step wise increase in the number of nucleated voids is observed. On the other hand, along RD direction that behavior is not present. This might be due to the morphological difference of the martensite phase.

Figure 4.15 shows the decrease in average nearest neighbor distance, which essentially shows the closeness of two voids. This factor has a direct relation to the coalescence of voids. During deformation, it is observed that the nearest neighbor distance decreases in a linear fashion in RD, whereas in TD it first decreases down to a certain point with an increasing rate, after which it slows down. The final distance is smaller in the RD sample which is also linked to the larger number of voids in RD.

To summarize, in both straining directions, voids are observed before the macroscopic necking of the samples. With further deformation both the number and the total area of the voids increase. The rate of void nucleation and growth is initially rather small, but it increases rapidly. Moreover, inter-void spacing decreases in a linear fashion: this is related to nucleation of new voids, the growth of existing voids and a decrease in the thickness of the specimens. 


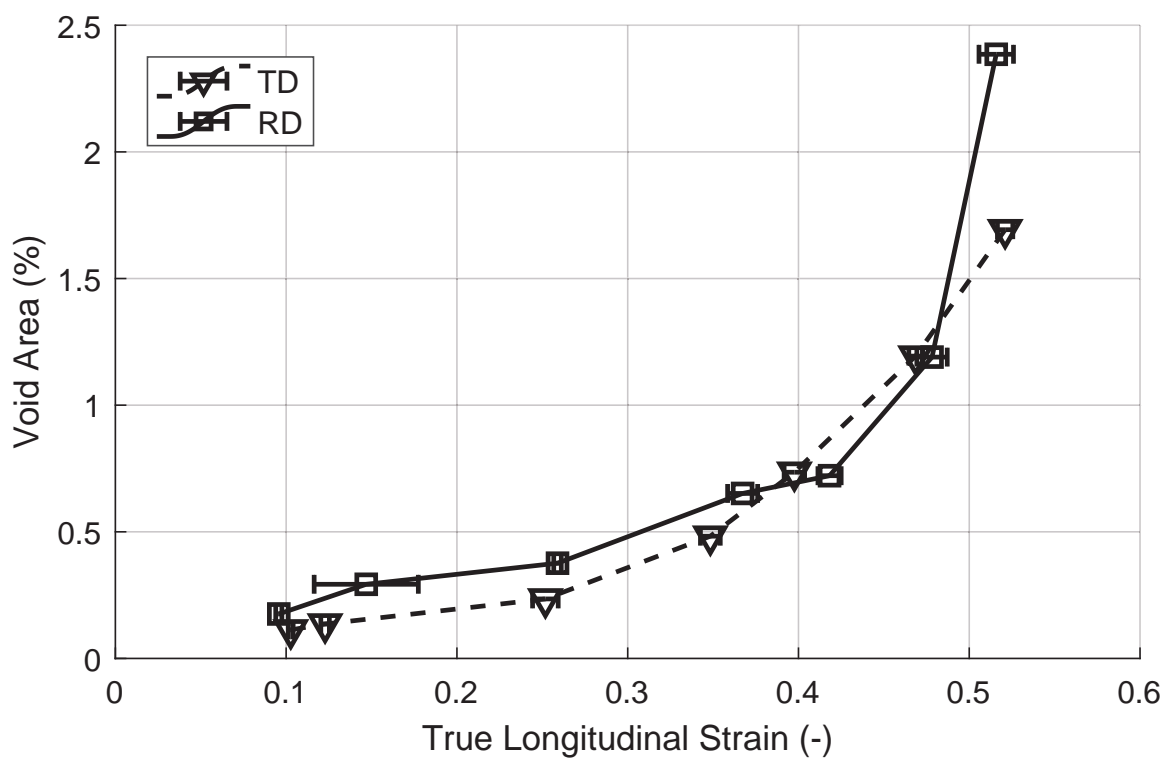

Figure 4.11: Void evolution in terms of $\%$ area.

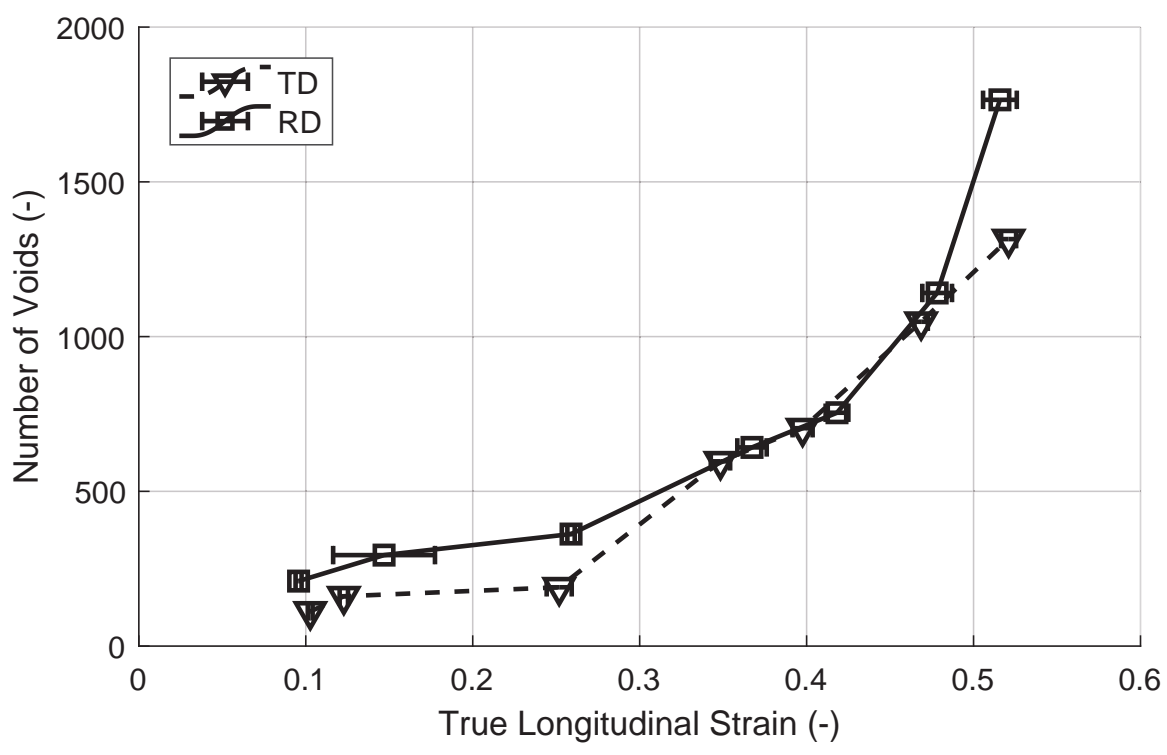

Figure 4.12: Number of voids in $0.18 \mathrm{~mm}^{2}$.

\subsubsection{Void distribution}

In this section, the damage in the samples is investigated in terms of the distribution of the damaged area across the thickness with respect to the loading direction. 


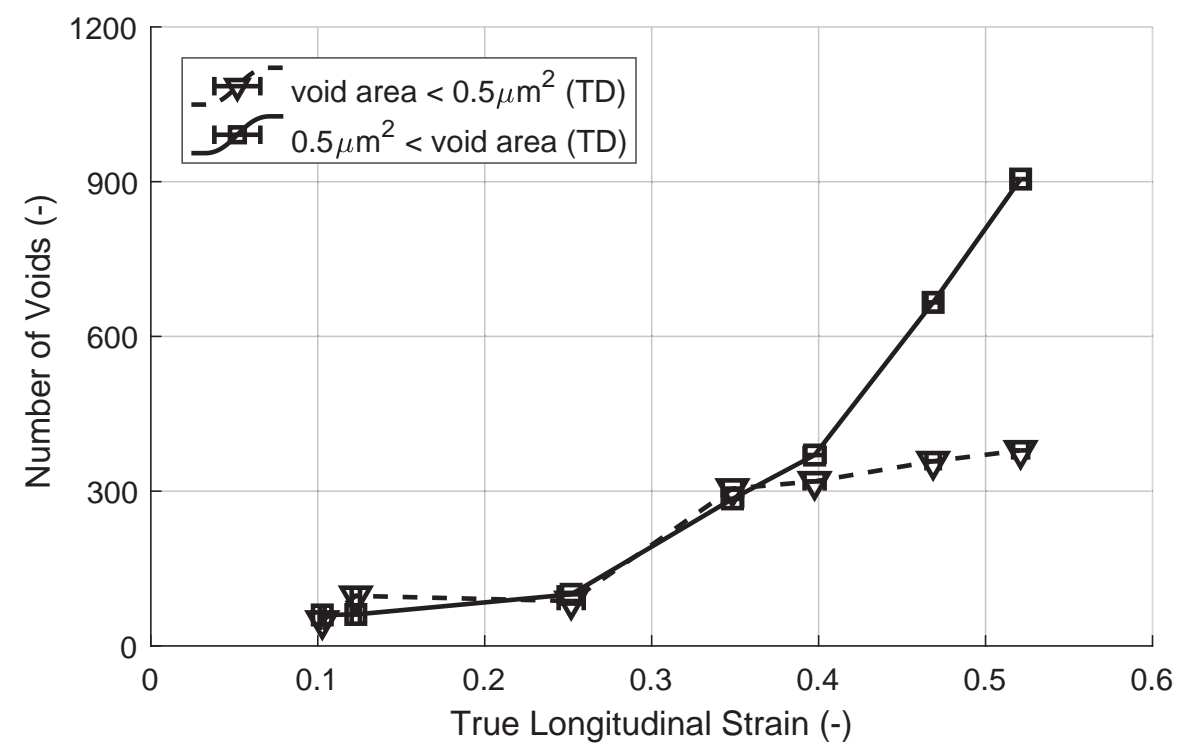

Figure 4.13: Void nucleation vs void growth in samples loaded in transverse direction.

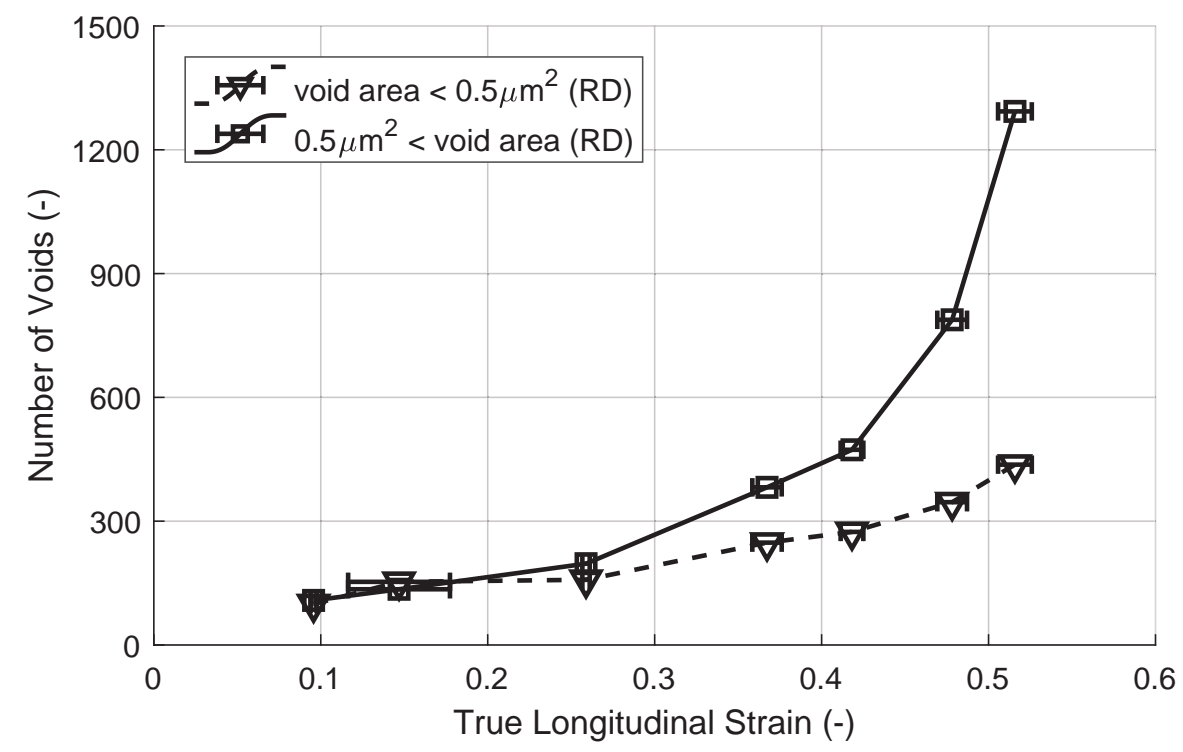

Figure 4.14: Void nucleation vs void growth in samples loaded in rolling direction.

Figures 4.16 and 4.17 show the identified voids and their spatial distribution in the most strained specimens. The graphs on the left of the secondary electron images show distribution of voids along the thickness, whereas the graphs under the images 


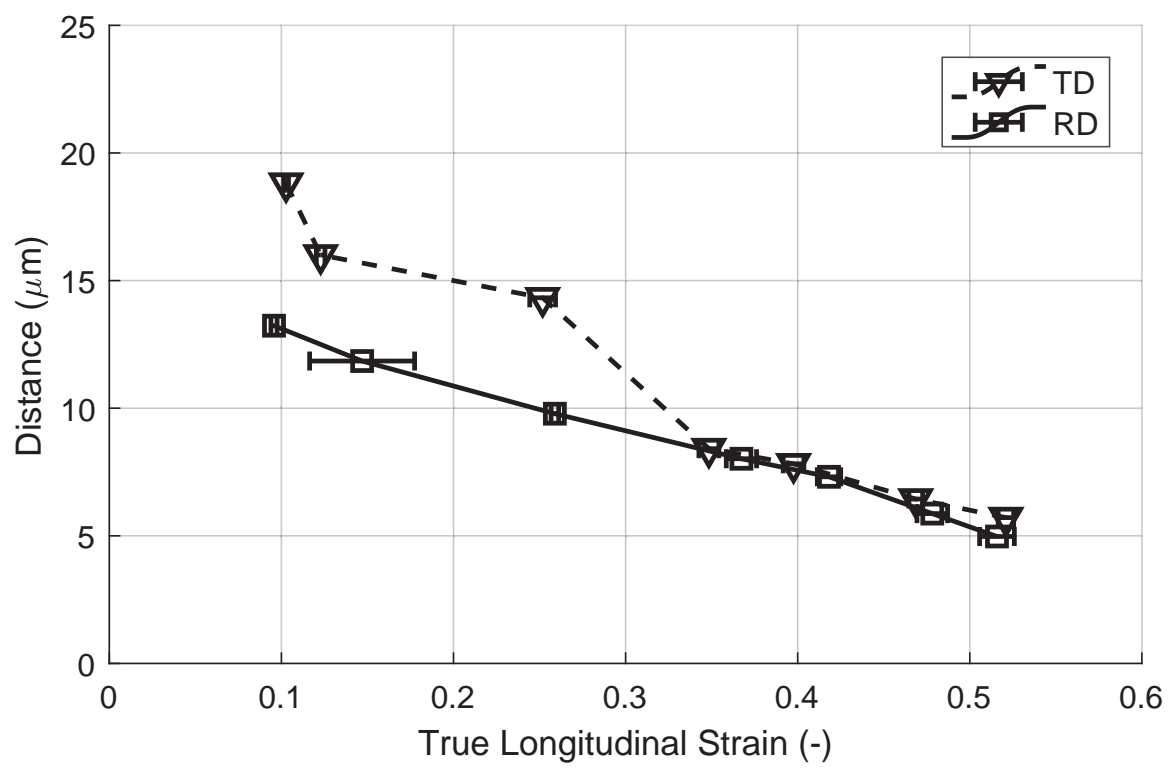

Figure 4.15: Change of the average nearest neighbor distance

represent the void distribution along the loading direction. It is observed that along the loading direction the distribution of the damaged area is quite homogeneous whereas along the thickness direction it is highly nonuniform. In addition, a clear large amount of damage concentration around the mid-plane stands out. The size of the peak is larger in RD than that of TD.

Figure 4.18 shows the evolution of the spatial void distribution along the thickness with an increasing strain in samples loaded in the TD (left column) and the RD (right column). At the initial interruption points, void distribution evolves homogeneously for both of the loading directions. Subsequent deformation causes bands with high concentration of voids especially at the center, where thicker martensite bands are observed. In samples loaded along the rolling direction, the highest void concentration occurred at a centrally located single band, whereas in those loaded along the transverse direction multiple bands are observed along the thickness of the specimen, as seen in the last row of Figure 4.18. This can be attributed to the continuity of martensite bands along rolling direction. It is also possible to make a similar observation by comparing identified voids in the white boxes of Figure 4.16 and 4.17. This might be caused by the difference in martensite band continuity. In RD, the central martensite band is longer and more continuous than TD due to rolling operations. One deviation from the general behavior is the $2^{\text {nd }}$ interruption of sample loaded along $\mathrm{RD}$ where the high peak was caused by an inclusion related damage incident. 

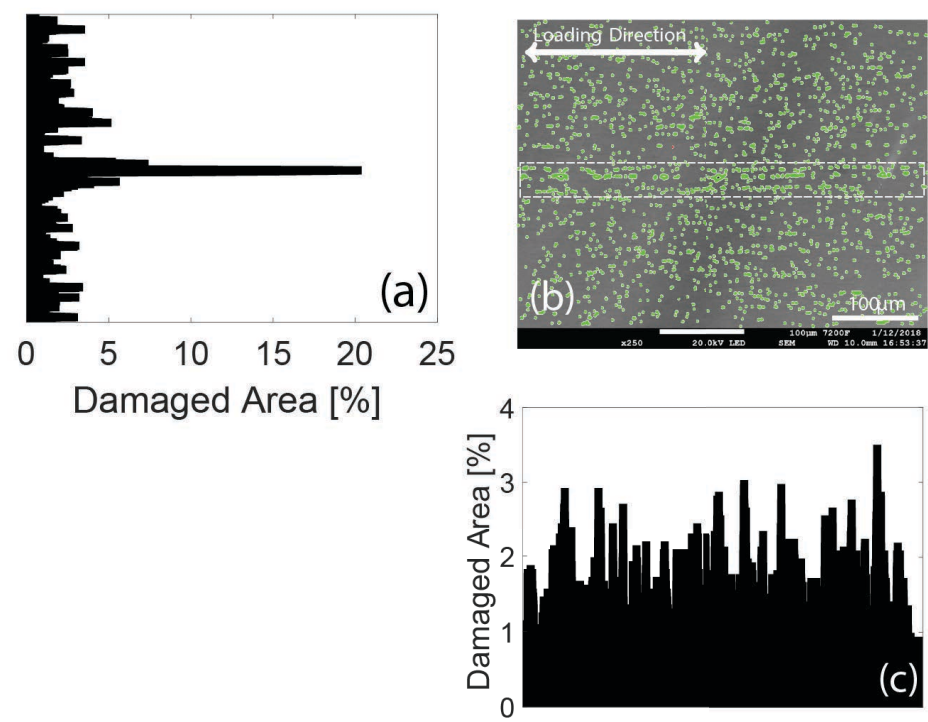

Figure 4.16: Void distribution in RD sample, a)void distribution along the thickness direction, b)identified voids, c)void distribution along the loading direction. The white dashed boxes indicate regions where voids are distributed along a band.
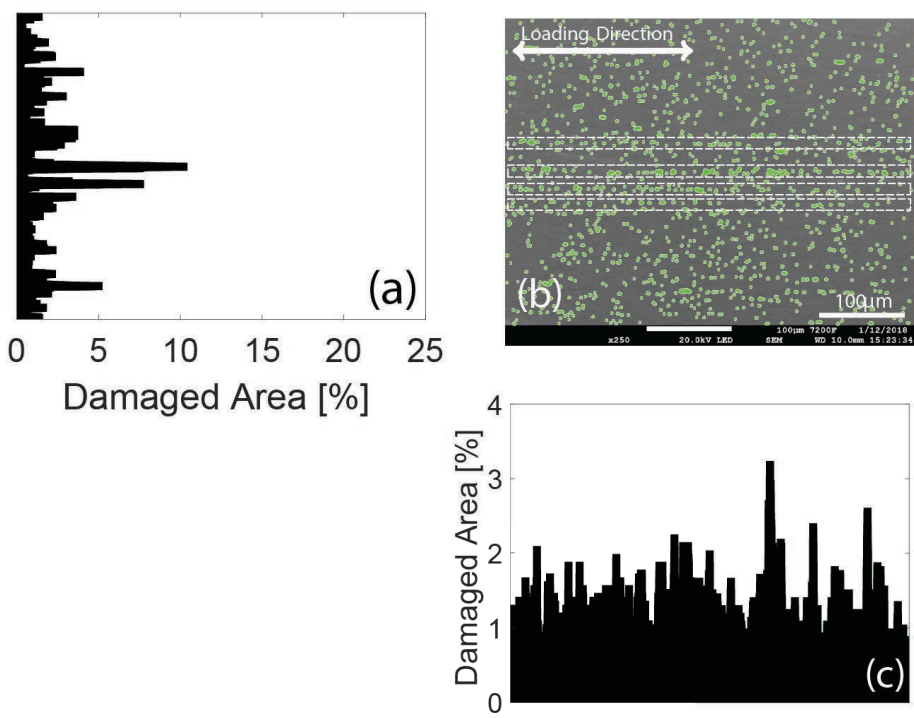

Figure 4.17: Void distribution in TD sample, a)void distribution along thickness direction, b)identified voids, c)void distribution along loading direction. The white dashed boxes indicate regions where voids are distributed along a band. 


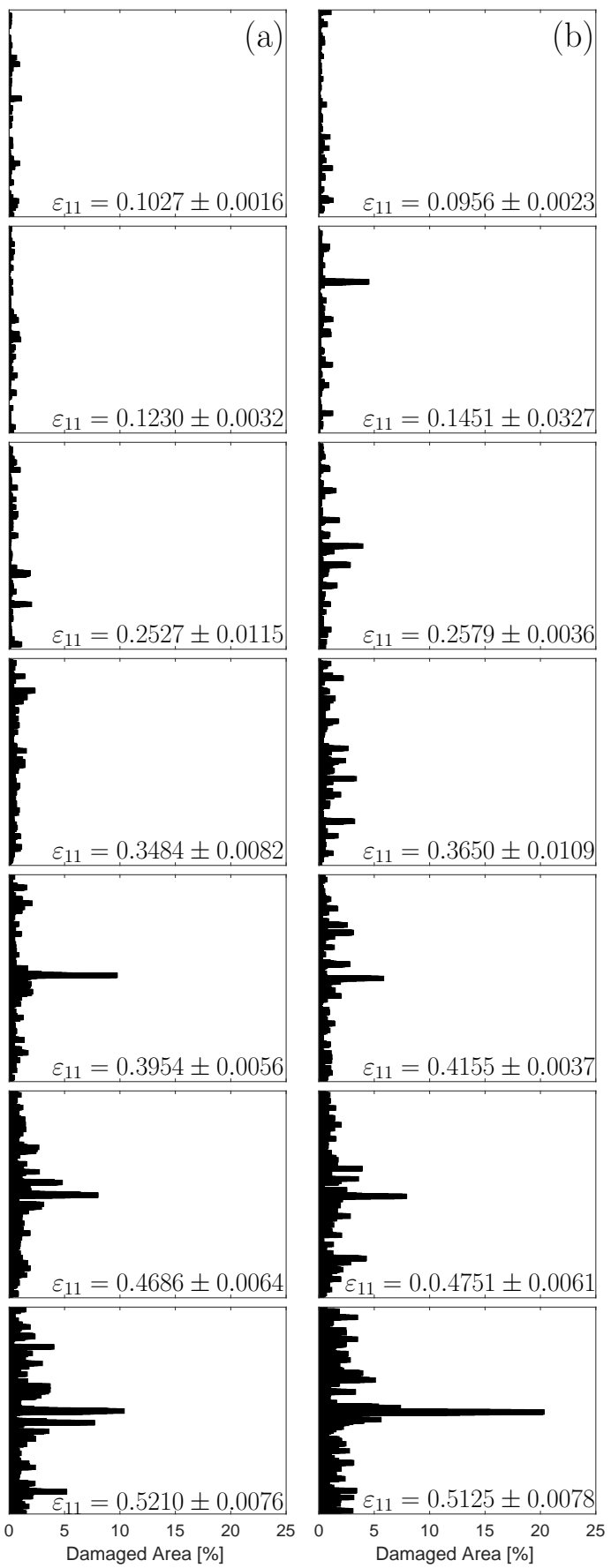

Figure 4.18: Void distribution along thickness direction with increasing strain, a)TD, b)RD samples. 


\subsection{Conclusions}

In this chapter, the focus was on investigating the active damage mechanisms in a commercial DP steel grade, both qualitatively and quantitatively, with special emphasis on the anisotropic nature of evolution of voids. To this end, tensile tests were carried out in TD and RD and the voids were observed using a combination of DIC and proposed image analysis steps which provide reproducible and objective quantification. Three active damage mechanisms, in terms of where voids nucleate, were identified, in line with literature. These are nucleation at inclusions, martensite cracking and decohesion at the ferrite-martensite interface. It is also observed that voids nucleate before UTS is reached.

The evolution of damage is characterized by interrupted tests where the damaged area and the number of voids are tracked separately. A strong correlation between these two quantities is found. At one level deeper however, it can be observed that the nucleation rate is slower than the growth rate.

The spatial distribution of voids is investigated and it is found that the average nearest neighbor distance decreases, as expected, with increasing strain. However, the rate at which this occurs is found to be dependent also on the loading direction.

Finally, it is found that the void evolution across the thickness direction is not uniform due to the banded nature of the martensite phase. The morphology of the band is direction dependent and since the main void nucleation mechanism is found to be martensite cracking, this leads to an anisotropy with respect to the loading direction. In the stronger banded direction, along $\mathrm{RD}$, voids are found to nucleate more inhomogeneously. 



\section{Modelling Framework: Strain gradient enhanced crystal plasticity}

This chapter aims to summarize the theory and implementation of a rate independent crystal plasticity model and the strain gradient enhancement. First, in Section 5.2, the kinematic model of crystal plasticity and geometrically necessary dislocation density is elaborated. Next, in Section 5.3 the details on implementation of the framework is introduced. Finally, in Section 5.4 two example problems, torsion of a disc and grain size strengthening, are illustrated. These problems show the strain gradient effects resulting from the structural gradient of strain as well as the gradient that is caused by the inhomogeneous microstructural deformation.

\subsection{Introduction}

In metals, the primary mechanism of inelastic deformation is plastic slip due to dislocation movement along glide planes. Due to the inhomogeneous nature of the microstructure, however, this movement is hindered by obstacles causing increase resistance to slip. According to [89] the magnitude of resistance depends on the number and type of obstacles. Since during deformation at low temperatures (with respect to the melting point of the metal) most microstructural features such as the grain morphology, number and composition of inclusions, the chemical composition

Some parts are based on:

ES Perdahcıoğlu, C Soyarslan, EE Aşık, AH van den Boogaard and S Bargmann

A class of rate-independent lower-order gradient plasticity theories: Implementation and application to disc torsion problem. Materials, 11(8) ,1425, 2018.

EE Aşık, ES Perdahcıoğlu, and AH van den Boogaard.

An RVE based study of the effect of martensite banding on damage evolution in dual phase steels. Computational Materials Science, (submitted). 
of phases can be assumed to remain unaltered, the main mechanism that contributes to the change of resistance is the net change in the density of immobile dislocations causing dislocation pile-up [90,91].

During plastic slip, this number increases due to generation mechanisms such as Frank-Read sources and decreases due to dislocation annihilation where usually the net effect remains non-negative (at low temperatures where recovery and recrystallization are negligible). These types of dislocations are commonly referred to as statistically stored dislocations (SSDs) due to the statistically random nature of their generation. This randomness results in a vanishing closed-circuit evaluation of the Burgers vector [92].

Another source of dislocations is geometrical necessity, as proposed by [48]. When large strain gradients prevail in the material an incompatibility of the crystal lattice arises which can be seen as a non-vanishing Burgers vector over a closed circuit. This implies that dislocations with certain orientations must be generated in order to reduce elastic distortion of the lattice. This type of dislocations is commonly referred to as geometrically necessary dislocations (GNDs).

However, apart from their sources, both types of dislocations, locally, are in essence the same and, hence, contribute to the hardening of the material similarly in terms of creating dislocation pile-ups.

If the thermodynamics of the total deformation is considered, two types of state variables, namely the plastic slip and its gradient, contribute to the dissipation of free energy of the system. In other words, both terms can be considered as thermodynamic fluxes which must have associated thermodynamic forces. The force associated with slip is the Cauchy stress itself in the macroscopic approach and the critical resolved shear stress in the microscopic definition. For the gradient of slip, the associated thermodynamic force is the microscopic stress [93-95]. The presence of an additional dissipative mechanism demands further external forces to drive deformation. This corresponds to plastic hardening. For this type of models in which the plastic strain or the slip rates are defined as individual degrees of freedom, it is also necessary to define a constitutive law between the microscopic stress and the respective gradient term.

In the literature, based on the consideration of the microscopic stresses in the definition of the (micro)equilibrium, there are mainly two approaches regarding strain gradient plasticity. According to the terminology defined in [96], the models that do take this into account are referred to as higher-order and those who relate the enhanced hardening to GNDs only are referred to as lower-order approaches.

In the higher-order approach, in addition to microscopic stresses, additional boundary conditions related to the added degrees of freedom must be defined with respect to the weak-form of the boundary value problem. At the boundaries, which can also be the grain boundaries for the crystal plasticity framework, this gives more flexibility in defining realistic phenomena such as permeability of dislocations across the grain boundaries. On the other hand, inaccurate description of the physics at the boundaries of a structure may result in peculiar and even counterintuitive behavior [97]. 
In previous research on gradient enhanced crystal plasticity models mostly ratedependent theories have been introduced [98-107] with some exceptions such as [108-112]. In this study, a rate-independent crystal plasticity formulation is used, in which, slip occurs only on slip planes where the resolved shear stress value is larger than the slip resistance [113]. The hardening on each individual slip system is described by the total dislocation density. GND densities are computed by projecting the slip gradient on each system, on the slip vectors, further classified into edge or screw types. The evolution of SSDs are modeled based on observations of their generation mechanisms. The contribution of the total dislocation densities to the slip resistance on their respective as well as related slip systems are obtained using an interaction matrix. Finally, Taylor type of hardening relation is utilized in relating the total dislocation densities to the critical resolved shear stress.

The gradients of slip and plastic strain are computed using an explicit formulation after each converged time step in an implicit, non-linear FE simulation. The result is a weak coupling of the slip gradient and the equilibrium solution. Accordingly, within each increment a small error is introduced that is corrected in the following step.

\subsection{Theory}

\subsubsection{Kinematics}

In the current model, it is assumed that crystallographic slip is the only deformation mechanism responsible for plastic deformation which happens on slip systems. The slip systems are defined by unit vectors of slip direction $\left(\mathbf{s}_{0}^{(\alpha)}\right)$ and slip plane normal $\left(\mathbf{m}_{0}^{(\alpha)}\right)$ of the slip system $\alpha$. The total deformation (elastic and inelastic) can be expressed by using the total deformation gradient $\mathbf{F}$ which takes a line element of the material $\mathrm{d}_{0}$ from an undeformed, reference configuration to $\mathrm{d} \mathbf{x}$ in the final deformed configuration. For finite deformation, multiplicative decompositions of the total deformation gradient can be used in the form

$$
\mathbf{F}=\hat{\mathbf{F}}_{\mathrm{e}} \cdot \mathbf{F}_{\mathrm{i}}
$$

where $\mathbf{F}_{\mathrm{i}}$ describes the inelastic deformation caused by glide of dislocations and $\hat{\mathbf{F}}_{\mathrm{e}}$ describes elastic stretching and lattice rotation of the plastically deformed material point at an intermediate configuration. The total velocity gradient $\mathbf{L}$ is calculated as

$$
\begin{gathered}
\mathbf{L}=\dot{\mathbf{F}} \cdot \mathbf{F}^{-1}=\left(\dot{\hat{\mathbf{F}}}_{\mathrm{e}} \cdot \mathbf{F}_{\mathrm{i}}+\hat{\mathbf{F}}_{\mathrm{e}} \cdot \dot{\mathbf{F}}_{\mathrm{i}}\right) \cdot \mathbf{F}_{\mathrm{i}}^{-1} \cdot \hat{\mathbf{F}}_{\mathrm{e}}^{-1} \\
\mathbf{L}=\hat{\mathbf{L}}_{\mathrm{e}}+\hat{\mathbf{F}}_{\mathrm{e}} \cdot \mathbf{L}_{\mathrm{i}} \cdot \hat{\mathbf{F}}_{\mathrm{e}}^{-1}=\hat{\mathbf{L}}_{\mathrm{e}}+\hat{\mathbf{L}}_{\mathrm{i}}
\end{gathered}
$$

The inelastic part of the total velocity gradient tensor is calculated by the sum of shear rates $\left(\dot{\gamma}^{(\alpha)}\right)$ of the slip systems as $[114,115]$

$$
\mathbf{L}_{\mathrm{i}}=\sum_{\alpha} \dot{\gamma}^{(\alpha)} \mathbf{s}_{0}^{(\alpha)} \otimes \mathbf{m}_{0}^{(\alpha)} \quad, \quad \hat{\mathbf{L}}_{\mathrm{i}}=\sum_{\alpha} \dot{\gamma}^{(\alpha)} \mathbf{s}^{(\alpha)} \otimes \mathbf{m}^{(\alpha)}
$$




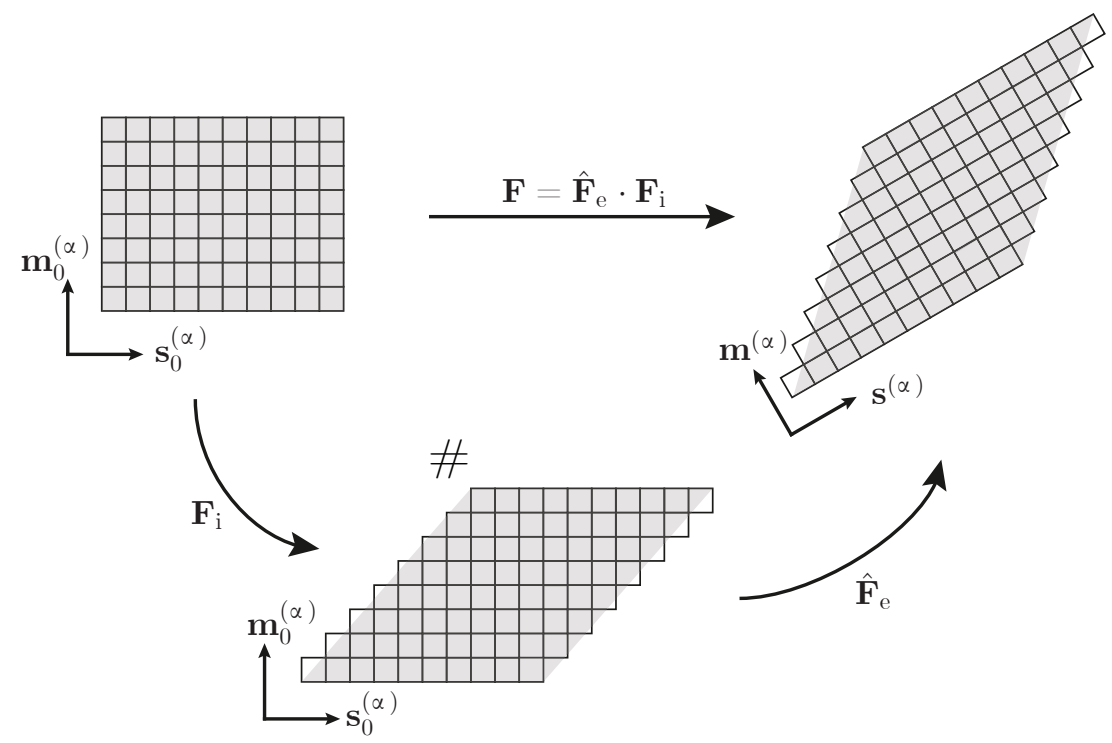

Figure 5.1: Geometric setting of multiplicative decomposition of the deformation gradient.

where

$$
\mathbf{s}^{(\alpha)}=\hat{\mathbf{F}}_{\mathrm{e}} \cdot \mathbf{s}_{0}^{(\alpha)} \quad, \quad \mathbf{m}^{(\alpha)}=\hat{\mathbf{F}}_{\mathrm{e}}^{-\mathrm{T}} \cdot \mathbf{m}_{0}^{(\alpha)}
$$

Furthermore by using the additive decomposition of the velocity gradient into rate of deformation $(\mathbf{D})$ and spin tensor $(\mathbf{W})$, from Eq. (5.3) one can obtain

$$
\mathbf{L}=\hat{\mathbf{D}}_{\mathrm{e}}+\hat{\mathbf{F}}_{\mathrm{e}} \cdot \mathbf{D}_{\mathrm{i}} \cdot \hat{\mathbf{F}}_{\mathrm{e}}^{-1}+\hat{\mathbf{W}}_{\mathrm{e}}+\hat{\mathbf{F}}_{\mathrm{e}} \cdot \mathbf{W}_{\mathrm{i}} \cdot \hat{\mathbf{F}}_{\mathrm{e}}^{-1}
$$

where:

$$
\begin{aligned}
\mathbf{D} & =\hat{\mathbf{D}}_{\mathrm{e}}+\hat{\mathbf{F}}_{\mathrm{e}} \cdot \mathbf{D}_{\mathrm{i}} \cdot \hat{\mathbf{F}}_{\mathrm{e}}^{-1}=\hat{\mathbf{D}}_{\mathrm{e}}+\hat{\mathbf{D}}_{\mathrm{i}} \\
\mathbf{W} & =\hat{\mathbf{W}}_{\mathrm{e}}+\hat{\mathbf{F}}_{\mathrm{e}} \cdot \mathbf{W}_{\mathrm{i}} \cdot \hat{\mathbf{F}}_{\mathrm{e}}^{-1}=\hat{\mathbf{W}}_{\mathrm{e}}+\hat{\mathbf{W}}_{\mathrm{i}}
\end{aligned}
$$

Here, a common simplification is introduced on $\hat{\mathbf{F}}_{\mathrm{e}}$ such that $\hat{\mathbf{R}}_{\mathrm{e}} \gg \hat{\mathbf{U}}_{\mathrm{e}}$ and $\hat{\mathbf{U}}_{\mathrm{e}} \cong \mathbf{I}$ since the elastic stretch of a metal is rather small. This implies that the length of unit vectors $\mathbf{s}_{0}^{(\alpha)}$ and $\mathbf{m}_{0}^{(\alpha)}$ do not change during deformation but only rotate with $\hat{\mathbf{R}}_{\mathrm{e}}[45,50,116,117]$ which simplifies Eq. (5.7) to

$$
\begin{aligned}
\mathbf{D} \cong \hat{\mathbf{D}}_{\mathrm{e}}+\hat{\mathbf{R}}_{\mathrm{e}} \cdot \mathbf{D}_{\mathrm{i}} \cdot \hat{\mathbf{R}}_{\mathrm{e}}^{-1} \cong \hat{\mathbf{D}}_{\mathrm{e}}+\hat{\mathbf{D}}_{\mathrm{i}} \\
\mathbf{W} \cong \hat{\mathbf{W}}_{\mathrm{e}}+\hat{\mathbf{R}}_{\mathrm{e}} \cdot \mathbf{W}_{\mathrm{i}} \cdot \hat{\mathbf{R}}_{\mathrm{e}}^{-1} \cong \hat{\mathbf{W}}_{\mathrm{e}}+\hat{\mathbf{W}}_{\mathrm{i}}
\end{aligned}
$$


In a finite element based time integration scheme the incremental rotation $(\Delta \mathbf{R})$ can be calculated from the exponential form of the known spin tensor $(\mathbf{W})$ as $[118]$

$$
\Delta \mathbf{R}=\exp (\mathbf{W}) \cong\left(\mathbf{I}+\frac{1}{2} \mathbf{W}\right) \cdot\left(\mathbf{I}-\frac{1}{2} \mathbf{W}\right)^{-1}
$$

and the total rotation at the end of the time step $(t+1)$ can be obtained from

$$
\mathbf{R}^{t+1}=\Delta \mathbf{R} \cdot \mathbf{R}^{t}
$$

With this, and considering that the total spin during the increment is known, the total elastic rotation of the slip systems can now be found using [119] :

$$
\hat{\mathbf{R}}_{\mathrm{e}}^{t+1}=\exp \left(\hat{\mathbf{W}}_{\mathrm{e}}\right) \cdot \hat{\mathbf{R}}_{\mathrm{e}}^{t}=\exp \left(\mathbf{W}-\hat{\mathbf{W}}_{\mathrm{i}}\right) \cdot \hat{\mathbf{R}}_{\mathrm{e}}^{t}
$$

\subsubsection{Flow rule}

In the rate-independent formulation, slip occurs only on the slip systems where the resolved shear stress $\left(\tau^{(\alpha)}\right)$ of the slip system $\alpha$ is greater than its slip resistance $\left(\tau_{\mathrm{f}}^{(\alpha)}\right)$. Hence, we can define $\phi^{(\alpha)}$ for each slip system as:

$$
\phi^{(\alpha)}=\tau^{(\alpha)}-\tau_{\mathrm{f}}^{(\alpha)}
$$

The systems for which $\phi^{(\alpha)}>0$ are called active slip systems and in the formulation all the shear rates $\dot{\gamma}^{(\alpha)}$ are defined to be positive. This necessitates consideration of opposite slip directions separately and the inactive slip systems do not take part in plastic deformation [113].

The elastic behavior in the global frame is defined by the relation between lattice corotational rate of Cauchy stress tensor and rate of elastic deformation as:

$$
\stackrel{\nabla}{\boldsymbol{\sigma}}=\mathbb{C}^{\mathrm{e}}: \mathbf{D}^{\mathrm{e}}
$$

At each time increment, ABAqus/STANDARD rotates the stress tensor $\boldsymbol{\sigma}$ to the global reference frame in corotational formulation [120,121].

The resolved stress on the slip system $\alpha$ is calculated by the projection

$$
\tau^{(\alpha)}=\boldsymbol{\sigma}: \mathbf{P}_{\mathrm{tot}}^{(\alpha)}
$$

where $\boldsymbol{\sigma}$ is the Cauchy stress tensor and $\mathbf{P}_{\text {tot }}^{(\alpha)}$ is called total Schmid tensor, defined as:

$$
\mathbf{P}_{\mathrm{tot}}^{(\alpha)}=\left\{\begin{array}{cc}
\mathbf{P}_{\mathrm{s}}^{(\alpha)} & \text { for FCC } \\
\mathbf{P}_{\mathrm{s}}^{(\alpha)}+\mathbf{P}_{\mathrm{ns}}^{(\alpha)} & \text { for BCC }
\end{array}\right.
$$


The $\mathbf{P}_{\mathrm{S}}^{(\alpha)}$ component of $\mathbf{P}_{\text {tot }}^{(\alpha)}$ geometrically projects the applied stress on the slip system with the slip direction $\mathbf{s}^{(\alpha)}$ and slip plane normal $\mathbf{m}^{(\alpha)}$.

$$
\mathbf{P}_{\mathbf{s}}^{(\alpha)}=\mathbf{s}^{(\alpha)} \otimes \mathbf{m}^{(\alpha)}
$$

$\mathbf{P}_{\text {ns }}^{(\alpha)}$ describes the non-Schmid effects that are responsible for the tensile compression asymmetry behavior of body centered cubic (BCC) crystals due to the core structure of screw dislocations and is calculated as [122]:

$$
\mathbf{P}_{\mathrm{ns}}^{(\alpha)}=\mathrm{a}_{1}\left(\mathbf{s}^{(\alpha)} \otimes \mathbf{m}_{\mathrm{l}}^{(\alpha)}\right)+\mathrm{a}_{2}\left(\mathbf{m}^{(\alpha)} \times \mathbf{s}^{(\alpha)}\right) \otimes \mathbf{m}_{\mathrm{l}}^{(\alpha)}+\mathrm{a}_{3}\left(\mathbf{m}_{\mathrm{l}}^{(\alpha)} \times \mathbf{s}^{(\alpha)}\right) \otimes \mathbf{m}_{\mathrm{l}}^{(\alpha)}
$$

where $a_{1}, a_{2}, a_{3}$ are temperature dependent material parameters. In the literature, non-Schmid behavior of BCC structures has been investigated extensively by many researchers and the reader is referred to these works [122-129]. Here we adopt the formulation developed by Gröger et al. [122] and employed by Koester et al. [130] and Cereceda et al. [126]. In this formulation, Eq. (5.17), the vector $\mathbf{m}_{1}^{(\alpha)}$ is the normal of non-glide plane which forms an angle of $30^{\circ}$ with the glide plane normal $\mathbf{m}^{(\alpha)}$. List of the vectors $\mathbf{s}^{(\alpha)}, \mathbf{m}^{((\alpha)}$ and $\mathbf{m}_{1}^{(\alpha)}$ can be found in the works of Gröger et al. [122] as well as Cereceda et al. [126]. Moreover, the material specific parameters are used as $\mathrm{a}_{1}^{298 \mathrm{~K}}=0.030, \mathrm{a}_{2}^{298 \mathrm{~K}}=0.173, \mathrm{a}_{3}^{298 \mathrm{~K}}=0.300$ from the works of Patra et al. [123] and Mapar et al. [131] in our calculations.

\subsubsection{Hardening rule}

In the current work, the main mechanism for work hardening was considered to happen by impediment of dislocation motion by increase in the forest dislocation density. Therefore for each slip system, a Taylor type hardening law [132] with physically based interaction matrix was employed as in Eq. (5.18):

$$
\tau_{\mathrm{f}}^{(\alpha)}=\tau_{0}+\mu b \sqrt{\sum_{\beta} Q^{(\alpha \beta)} \rho^{(\beta)}}
$$

where $\tau_{0}$ is the strain independent lattice friction, $\mu$ is the shear modulus, $b$ is the Burger vector length, $\rho^{(\beta)}$ is the total dislocation density of the slip system $\beta$ and $Q^{(\alpha \beta)}$ is a physically-based interaction matrix.

The coefficients of interaction matrix $Q^{(\alpha \beta)}$ characterizes the strengthening of slip system $\alpha$ due to increase of dislocation density on $\beta$. It is composed of six possible independent interactions of type: self, coplanar, collinear, orthogonal, glissile and sessile [126,133-135]. Table 5.1 shows coefficients of the interaction matrix determined by Queyreau et al. [135] via discrete dislocation dynamics simulations for a BCC structure. Different coefficients exist for FCC crystals. 
Table 5.1: Coefficients of interaction matrix $Q^{(\alpha \beta)}$ for BCC-Fe [135]

\begin{tabular}{cccccc}
\hline Self & Coplanar & Collinear & Orthogonal & Glissile & Sessile \\
\hline 0.009 & 0.009 & 0.72 & 0.05 & 0.09 & 0.06 \\
\hline
\end{tabular}

By following the arguments of Ashby [89], the total dislocation density $\rho^{(\alpha)}$ of a slip system $\alpha$ was considered to be the sum of statistically-stored dislocation $\rho_{\mathrm{SSD}}^{(\alpha)}$ and geometrically-necessary dislocation $\rho_{\mathrm{GND}}^{(\alpha)}$ densities.

$$
\rho^{(\alpha)}=\rho_{\mathrm{SSD}}^{(\alpha)}+\rho_{\mathrm{GND}}^{(\alpha)}
$$

The evolution of $\rho_{\mathrm{SSD}}^{(\alpha)}$ is governed by shear rate of slip system $\alpha$ whereas $\rho_{\mathrm{GND}}^{(\alpha)}$ evolves by the gradient of the shear rate and it will be discussed in the next section. As in the work of Perdahcioğlu et al. [136], the evolution of $\rho_{\mathrm{SSD}}^{(\alpha)}$ is based on a phenomenological constitutive law based on the linear ordinary differential equation Eq. (5.20)

$$
\dot{\rho}_{\mathrm{SSD}}^{(\alpha)}=\frac{\dot{\gamma}_{(\alpha)}}{\gamma^{\infty}}\left[\rho_{\mathrm{SSD}}^{\infty}-\rho_{\mathrm{SSD}}^{(\alpha)}\right]
$$

where the terms $\rho_{\mathrm{SSD}}^{\infty}$ and $\gamma^{\infty}$ are constants that control the values for saturation of statistically-stored dislocation density and the rate of saturation which are phenomenological descriptions of the balance between rate of dislocation production and annihilation [108].

\subsubsection{GND density: Strain gradient enhancement}

This section gives the formulation that is required to calculate the evolution of $\rho_{\text {GND }}^{(\alpha)}$ based on the gradient of slip rates. For calculating the evolution of geometrically necessary dislocation densities, following Gurtin et al. [137] and Cermelli et al. [138], we start by defining the Burgers tensor $\mathbf{G}$, which characterizes the closure failure of an initially closed referential circuit as

$$
\mathbf{G}=\mathbf{F}^{\mathrm{i}} \cdot \operatorname{Curl} \mathbf{F}^{\mathrm{i}}
$$

and plastically convected rate of $\mathbf{G}$ as [139]:

$$
\stackrel{\square}{\mathbf{G}}=\sum_{\alpha}\left(\nabla^{\#} \dot{\gamma} \times \mathbf{m}^{(\alpha)}\right) \otimes \mathbf{s}^{(\alpha)}
$$

where $\nabla^{\#} \dot{\gamma}$ can be viewed as the gradient of $\dot{\gamma}$ in the lattice configuration (\#) [137]. We can define a unit lattice vector $\mathbf{l}^{(\alpha)}$ as $\mathbf{l}^{(\alpha)}=\mathbf{m}^{(\alpha)} \times \mathbf{s}^{(\alpha)}$. Then, by using the 
orthogonality of $\left(\nabla^{\#} \dot{\gamma} \times \mathbf{m}^{(\alpha)}\right)$ to $\mathbf{m}^{(\alpha)}, \stackrel{\square}{\mathbf{G}}$ can be expanded as

$$
\stackrel{\square}{\mathbf{G}}=\sum_{\alpha}\left(\mathbf{l}^{(\alpha)} \cdot \nabla^{\#} \dot{\gamma}^{(\alpha)} \mathbf{s}^{(\alpha)} \otimes \mathbf{s}^{(\alpha)}-\mathbf{s}^{(\alpha)} \cdot \nabla^{\#} \dot{\gamma}^{(\alpha)} \mathbf{l}^{(\alpha)} \otimes \mathbf{s}^{(\alpha)}\right)
$$

By definition, both the direction of the Burgers vector and dislocation line direction is equal to $\mathbf{s}^{(\alpha)}$ for screw dislocations while for edge dislocations the direction of the Burgers vector is $\mathbf{s}^{(\alpha)}$ and the dislocation line direction is $\mathbf{l}^{(\alpha)}$. Then the rate of screw $(\odot)$ and edge $(\vdash)$ dislocation densities due to lattice incompatibility in a slip system $\alpha$ can be calculated from the dislocation line and the gradient of slip rate vector as:

$$
\begin{gathered}
\dot{\rho}_{\odot, \mathrm{GND}}^{(\alpha)}=\mathbf{l}^{(\alpha)} \cdot \nabla^{\#} \dot{\gamma}^{(\alpha)}, \\
\dot{\rho}_{\vdash, \mathrm{GND}}^{(\alpha)}=-\mathbf{s}^{(\alpha)} \cdot \nabla^{\#} \dot{\gamma}^{(\alpha)},
\end{gathered}
$$

However, this calculation has to be done in the lattice configuration. On the other hand, the use of USDFLD - Abaqus user subroutine for field calculations algorthmically limits the calculations to be done in either reference or deformed configuration. In order to push forward or pull back, one can employ the identities [138]

$$
\nabla^{\#} \dot{\gamma}^{(\alpha)}=\mathbf{F}_{\mathrm{i}}^{-\mathrm{T}} \cdot \nabla_{0} \dot{\gamma}^{(\alpha)}=\mathbf{F}_{\mathrm{e}}^{\mathrm{T}} \cdot \nabla \dot{\gamma}^{(\alpha)}
$$

Here our choice was to push forward from lattice configuration \# to reference configuration and yield the GND evolution equations as [137]

$$
\begin{array}{r}
\dot{\rho}_{\odot, \mathrm{GND}}^{(\alpha)}=\mathbf{l}^{(\alpha)} \cdot \mathbf{F}_{\mathrm{i}}^{-\mathrm{T}} \cdot \nabla_{0} \dot{\gamma}^{(\alpha)}=\mathbf{l}_{0}^{(\alpha)} \cdot \nabla \dot{\gamma}^{(\alpha)} \\
\dot{\rho}_{\vdash, \mathrm{GND}}^{(\alpha)}=-\mathbf{s}^{(\alpha)} \cdot \mathbf{F}_{\mathrm{i}}^{-\mathrm{T}} \cdot \nabla_{0} \dot{\gamma}^{(\alpha)}=-\mathbf{s}_{0}^{(\alpha)} \cdot \nabla \dot{\gamma}^{(\alpha)}
\end{array}
$$

The edge and screw GND densities represent the vector components of the total GND density and due to the definition of $\mathbf{G}$, the unit of densities is not per area but rather per length and it is more a geometrical measure without any material specific input. It is therefore necessary to convert to per area by dividing by the length of the Burgers vector of the material. This yields the total GND density to be used in Eq. (5.19) as

$$
\rho_{\mathrm{GND}}^{(\alpha)}=\frac{1}{\mathrm{~b}} \sqrt{\left[\rho_{\vdash, \mathrm{GND}}^{(\alpha)}\right]^{2}+\left[\rho_{\odot, \mathrm{GND}}^{(\alpha)}\right]^{2}}
$$

\subsection{Implementation}

\subsubsection{Rate-independent crystal plasticity}

The implementation of rate-independent crystal plasticity follows the same lines of conventional return mapping algorithms. A Backward-Euler approach is followed and 
the loading conditions are represented using the following Kuhn-Tucker conditions:

$$
\Delta \gamma^{(\alpha)} \geq 0, \quad \phi^{(\alpha)} \leq 0, \quad \Delta \gamma^{(\alpha)} \phi^{(\alpha)}=0 .
$$

Making use of the consistency condition the values for the incremental plastic multiplier on each slip system can be found with a suitable Newton-Raphson based iteration scheme.

$$
R^{(\alpha)}=\phi^{(\alpha)}=\boldsymbol{\sigma}(\boldsymbol{\gamma}):\left[\mathbf{s}^{(\alpha)} \otimes \mathbf{m}^{(\alpha)}\right]-\tau_{\mathrm{f}}^{(\alpha)}(\boldsymbol{\gamma}) .
$$

There are two points that must be considered in the implementation of rate independent crystal plasticity: non-uniqueness and active set determination. For both cases, we refer to robust and efficient solutions provided by $[113,117,140]$.

Non-uniqueness is a result of linear dependence of slip systems in case of isotropic hardening when the resolved shear stress on multiple slip systems are equal. This can, however, be circumvented by using Singular Value Decomposition (SVD) with which the correct slip amounts are recovered. In the study of Miehe et al., it was shown that perturbation methods could be used to approximate the SVD. In our implementation, we have employed the numerical perturbation technique due to its efficiency [113]. This method applies a constant shift to the linearization of Eq. (5.29) (A) in the Newton-Raphson iteration scheme such that the well-conditioned inverse can be written as:

$$
\overline{\mathbf{A}}^{-1}:=(\mathbf{A}+\epsilon \mathbf{I})^{-1},
$$

with the shift $\epsilon=\min C \cdot \max _{1 \leq \alpha \leq n}\left\{\sum_{\beta=1}^{n}\left|\mathbf{A}^{(\alpha \beta)}\right|\right\}$, where min $\mathrm{C}$ is the machine accuracy.

Secondly, the active set $\alpha$ is determined by the condition $\phi^{(\alpha)}>0$, which means Kuhn-Tucker conditions are violated. The issue that arises during iterations is that the active set might change as slip systems enter or leave the set due to the reduction in the trial stress. The change in the active set is realized by deactivation of the most violated system $\left(\dot{\gamma}^{(\alpha)}<0\right)$ which has $\phi^{(\alpha)}>0$.

As long as the correct systems that activate and deactivate can be found, the solution is converged. Here it is important to note that algorithmic slip is defined to be positive. In order to achieve this, slip along $\mathbf{s}^{(\alpha)}$ and slip along $-\mathbf{s}^{(\alpha)}$ have to be considered differently.

\subsubsection{Gradient computation}

In this work, strain gradient computation is realized explicitly by using a discrete gradient computation method proposed by Liszka and Orkisz [141], which is employed for gradient calculations in both isotropic and anisotropic plasticity [136,142]. This method allows computation of gradients for arbitrary irregular grids. Accordingly, the 


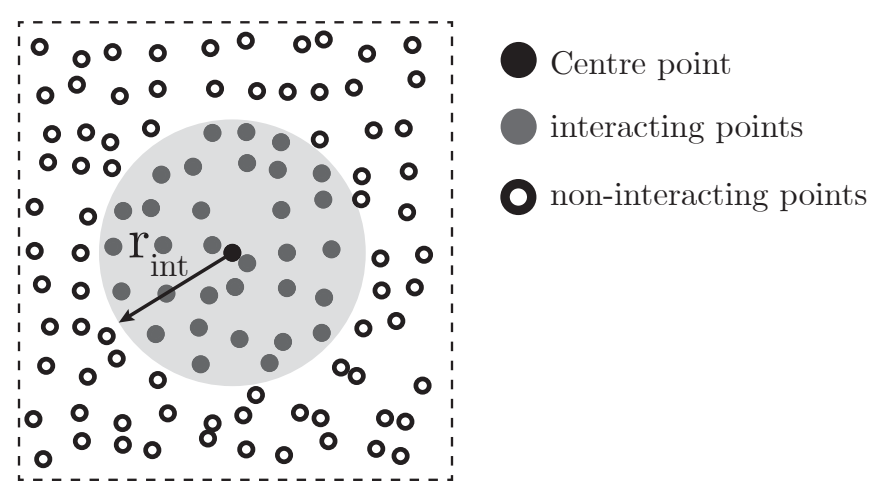

Figure 5.2: Schematic illustration of the interaction radius and the Gauß points in an irregular grid.

first order Taylor series expansion of $\dot{\gamma}^{(\alpha)}$ around a point $\mathbf{r}_{0}=\left\{x_{0}, y_{0}, z_{0}\right\}$ is developed as

$$
\dot{\gamma}^{(\alpha)}(\mathbf{r})=\dot{\gamma}^{(\alpha)}\left(\mathbf{r}_{0}\right)+\mathbf{\Upsilon} \cdot \Delta \mathbf{r}+O\left(\Delta x^{2}+\Delta y^{2}+\Delta z^{2}\right),
$$

and computed for the slip systems $\alpha=1,2,3, \ldots, N$ where $\boldsymbol{\Upsilon}$ represents the unknown gradient vector,

$$
\Upsilon=\left\{\frac{\partial \dot{\gamma}^{(\alpha)}\left(\mathbf{r}_{0}\right)}{\partial x}, \frac{\partial \dot{\gamma}^{(\alpha)}\left(\mathbf{r}_{0}\right)}{\partial y}, \frac{\partial \dot{\gamma}^{(\alpha)}\left(\mathbf{r}_{0}\right)}{\partial z}\right\}^{\mathrm{T}}
$$

and $\Delta \mathbf{r}$ is defined as $\Delta \mathbf{r}=\{\Delta x, \Delta y, \Delta z\}^{\mathrm{T}}$ with $\Delta x=x-x_{0}, \Delta x=y-y_{0}$ and $\Delta z=z-z_{0}$. Eq. (5.31) assumes that the macroscopic shearing rate $\dot{\gamma}^{(\alpha)}(\mathbf{r})$ is a sufficiently smooth and differentiable field that can be calculated at a point $\mathbf{r}=$ $\{x, y, z\}$. Writing eq. (5.31) for each Gauß point around a central Gauß point gives a set of linear equations. In order to increase the accuracy of approximation, the number of Gauß points, $n$, used exceeds the number of unknowns, which results in an overdetermined set of linear equations. In order to overcome the over-determinacy of this system as well as providing a smooth approximation to the gradient, the following form is used for the points within an interaction radius, $\mathrm{r}_{\mathrm{int}}$,

$$
f(\mathbf{\Upsilon})=\sum_{k=1}^{n}\left[\omega\left(\overline{\mathrm{r}}_{k}\right)\left(\dot{\gamma}^{(\alpha)}\left(\mathbf{r}_{0}\right)-\dot{\gamma}^{(\alpha)}\left(\mathbf{r}_{k}\right)+\mathbf{\Upsilon} \cdot \Delta \mathbf{r}_{k}\right)\right]^{2}
$$

where $\omega\left(\overline{\mathrm{r}}_{k}\right)$ is the weighting function in the form of $\left(\frac{\mathrm{r}_{\text {int }}}{\Delta r_{k}}\right)^{3}$ which decreases with increasing distance between the two Gauß points. Minimization of $f(\boldsymbol{\Upsilon})$ with $\partial f / \partial \Upsilon \mathbf{\Upsilon}=0$ gives the desired gradients. Fig. 5.2 shows schematically the points that are used for the calculation of Eq. (5.33) around the centre Gauß point. This procedure is implemented as a USDFLD subroutine for ABAQUS.

In the case of polycrystal simulations, the gradient computation is limited within each domain of elements belonging to individual material definitions. This implies that the 
jump of the plastic strain across the grain boundaries is not treated as a source of GNDs.

\subsubsection{Global solution scheme}

In the weakly coupled scheme that is presented above, the gradient effects do not directly influence the current equilibrium solution increment. On the other hand, the introduced GNDs (as a result of the increase in the gradients of plastic deformation) affect the hardening response of the material. This additional hardening term influences only the next increment in the equilibrium solver, making the current algorithm a staggered one. Using this type of incrementally staggered approach has examples in literature for non-local calculations [143,144].

The general equilibrium solution scheme with emphasis on the utilized user-defined subroutines (USDFLD, UMAT) in the commercial finite element software ABAQUS reads as:

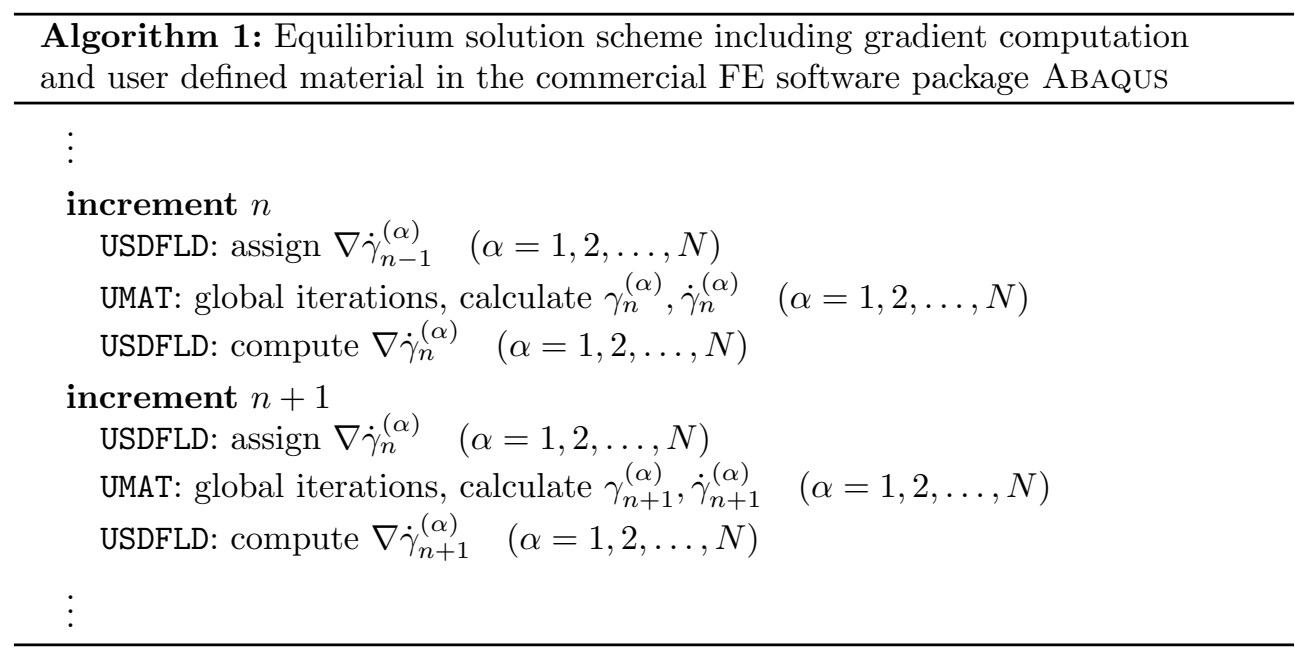

As seen in Alg. 1, the user defined field function is called twice, before and after global equilibrium, for each Gauß point. This gives the possibility to collect global data and do non-local calculations. In the first visit, USDFLD assigns the calculated gradients to integration points. In the second visit, it collects the new shear rates. When all the integration points are visited, shear rate gradients are calculated.

\subsection{Application}

In this section, two example problems, where gradient of strain has significant importance, are illustrated and elaborated within the framework for a generic face centered cubic crystal metal. The two examples focus on two aspects that causes 
plastic strain gradients, namely, geometry of loading and plastically inhomogeneous deformation of the material itself. The first example is torsion of a disc where twisting causes a macroscopically constant strain gradient from the core to the outer edge. This results in higher normalized torque values in smaller diameter discs. Moreover, this example intrinsically contains plastic inhomogenity due to the presence of lattice orientations. The second example shows grain size strengthening or the Hall-Petch effect where decreasing grain size increases plastic heterogeneity. The increase in fluctuation of strain field increases the strain gradient effects resulting in higher strength for smaller grains.

Since the aim of the simulations is to observe the gradient enhanced hardening behavior that arises from geometrical and microstructural conditions, the same material parameters are used for both examples. The material data used for both cases are summarized in Table 5.2. The stress strain curve in Figure 5.3 shows the tensile response of a $3 \mathrm{D}$ geometry with plane strain boundary conditions consisting of grains of $25 \mu \mathrm{m}$.

As a first observation from Figure 5.3, with increasing strain the gradient enhanced crystal plasticity model does not show a saturating type of behavior even if the SSD evolution saturates. This is due to the creation of GNDs which depend on the strain gradients and are, therefore, independent of the evolution of SSDs. This non-saturating behavior is also observed in experimental studies found in literature for aluminum as well as steel $[145,146]$.

\subsubsection{Torsion of a disc}

The geometry and boundary conditions considered in this test are depicted in Figure 5.4. Accordingly, in this test, the top and bottom sides of the disc are constrained and a twist is applied at the top. Wire torsion tests [93] suggest that as the disc radius decreases the additional hardening mechanism due to the applied strain gradient should become more dominant, resulting in a difference in the measured normalized torque $\left(\tau / r^{3}\right)$, which gives a measure of average shear stress across the section.

Table 5.2: Material data used in simulations for both examples.

\begin{tabular}{rll}
\hline & & Aluminum-like \\
\hline$E$ & $(\mathrm{GPa})$ & 75000 \\
$\nu$ & $(-)$ & 0.3 \\
$b$ & $(\mathrm{~mm})$ & $2.86 \times 10^{-7}$ \\
$\mathrm{c}$ & $(-)$ & 0.3 \\
$\rho_{0}$ & $\left(\mathrm{~mm}^{-2}\right)$ & $2 \times 10^{5}$ \\
$\rho^{\infty}$ & $\left(\mathrm{mm}^{-2}\right)$ & $3 \times 10^{8}$ \\
$\gamma^{\infty}$ & $(-)$ & 0.75 \\
$\tau_{0}$ & $(\mathrm{MPa})$ & 10 \\
$Q_{0-5}$ & $(-)$ & $2,10,10,10,8,15$ \\
\hline
\end{tabular}




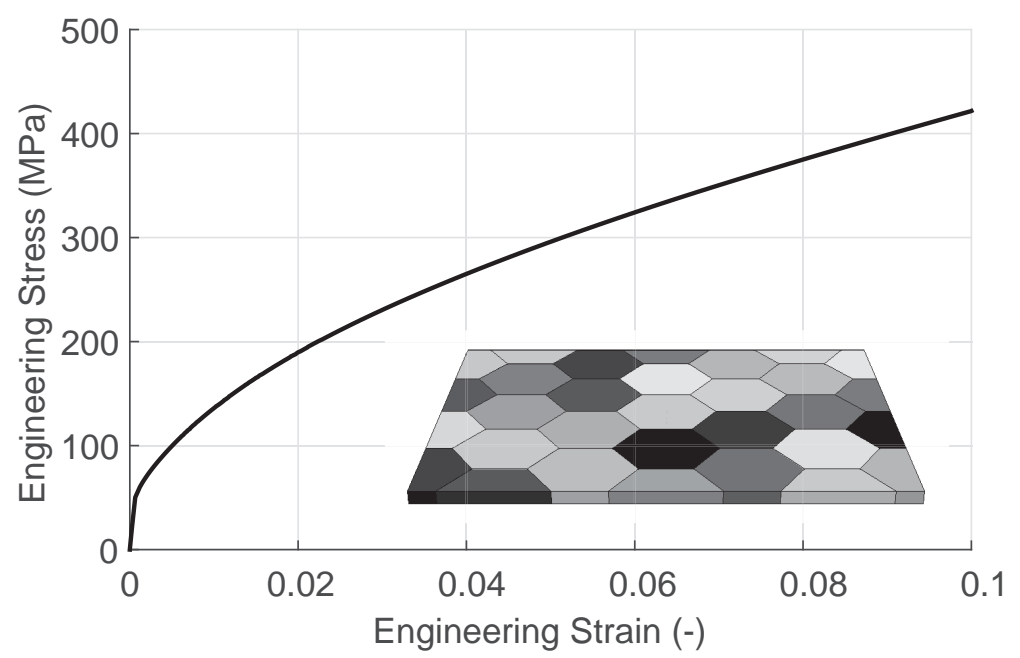

Figure 5.3: Numerical plane strain tension test and the hexagonal grain structure (different gray tones represent different grains).

The macroscopic shear component that is the main deformation mechanism is $\varepsilon_{\theta z}$ (see Figure 5.4) and it varies linearly in the radial direction when the rotations are prescribed on both surfaces. In addition to this structural gradient, the anisotropic response of the crystals naturally generates GNDs. This anisotropic response always exists, even if there is no structural gradient. The existence of grains with distinct lattice orientations creates strain gradients close to the grain boundaries. This results in an inherent hardening mechanism.
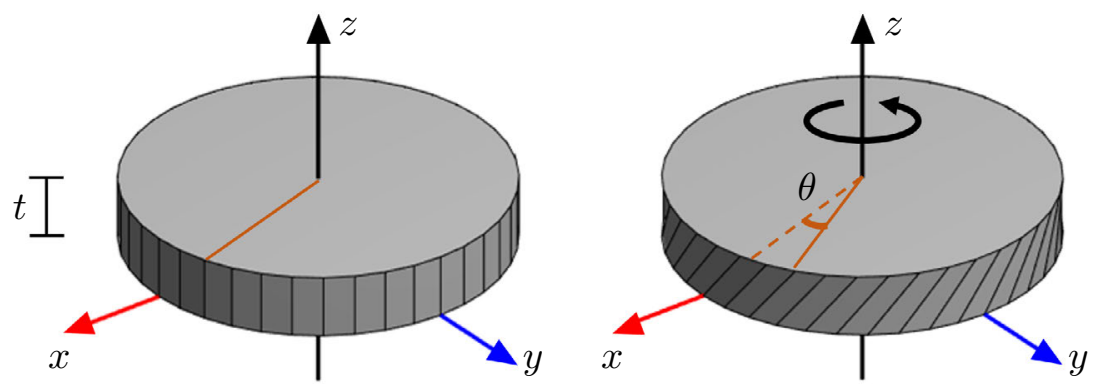

Figure 5.4: Considered disc during finite element simulations. Making use of cylindrical coordinates $(r, \theta, z)$ with $x=r \cos \theta, y=r \cos \theta$, during loading bottom surface with $z=0$ is fixed completely with $u_{r}(r, \theta, 0)=u_{\theta}(r, \theta, 0)=u_{z}(r, \theta, 0)=0$ whereas at the top surface with $z=t$, torsional loading along $z$-axis is applied with $u_{r}(r, \theta, t)=0, u_{\theta}(r, \theta, t)=\theta^{\prime}$ and $u_{z}(r, \theta, t)=0$.

As the disc diameter decreases, the grain size of the material remains constant if not 
explicitly altered. Due to the nature of the wire-drawing process, from which the disc is assumed to be a section, the grains in the disc are expected to be elongated in the primary axis of the wire. Therefore, the simulation models shown in Figure 5.5 are created in order to mimic the experiments as closely as possible. The approximate grain diameter is chosen to be $25 \mu \mathrm{m}$.

The structure is meshed using quadratic tetrahedral elements with 4 integration points and 10 nodes (C3D10). The same mesh size is used for all the simulations which yield elements with an average volume of $32 \mu \mathrm{m}^{3}$.

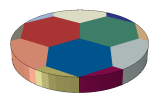

$r=25 \mu \mathrm{m}$

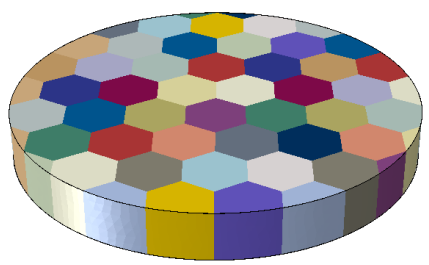

$r=75 \mu \mathrm{m}$

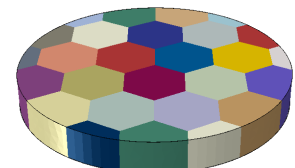

$r=50 \mu \mathrm{m}$

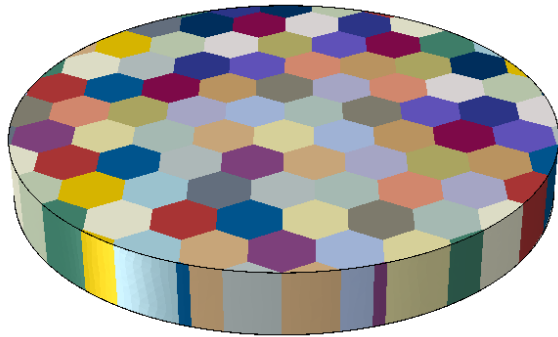

$r=100 \mu \mathrm{m}$

Figure 5.5: Microstructure of the discs used in the crystal plasticity simulations. Every crystal (represented by a different color) has a different crystallographic orientation.

The resulting normalized torque $\left(\tau / r^{3}\right)$ versus twist angle curves for the crystal plasticity case are plotted in Figure 5.6. Although there is only a minor amount of hardening down to a disc radius of $50 \mu \mathrm{m}$, below that a significant change occurs. One reason for this, as expected, is the increased strain gradient due to the boundary conditions. On the other hand, at these diameters only a few grains, hence crystallographic orientations, are available for generating slip, see Figure 5.5. Therefore, this simulation is repeated twice with randomly generated crystallographic orientations in order to observe the statistical nature of the hardening. The statistical size effect, as a result of highly constrained plastic deformation, becomes a significant mechanism for hardening as the disc radius decreases, see Figure 5.7. The resulting GND and SSD distributions for different disc diameters are shown in Figures 5.8 and 5.9, respectively. From Figure 5.9, it is seen that the due to the dependency of plastic deformation on lattice orientations, the SSD density shows complex distribution instead of a linear increase which would be predicted by isotropic plasticity models. More to that, also the GND density — since it is related to the 


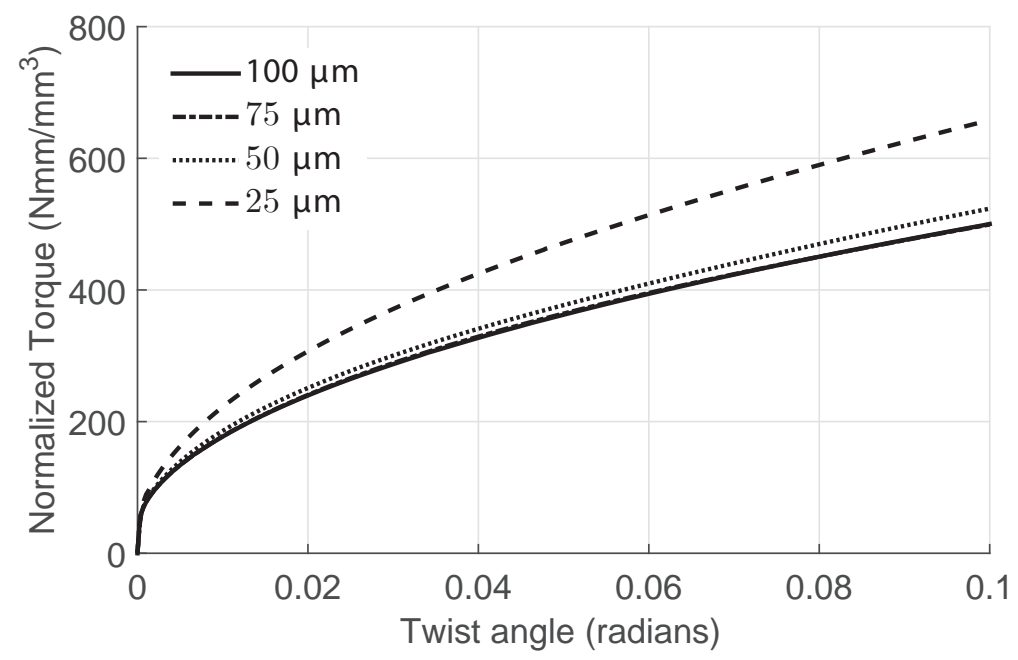

Figure 5.6: Torque vs. twist angle curves with varying disc radii.

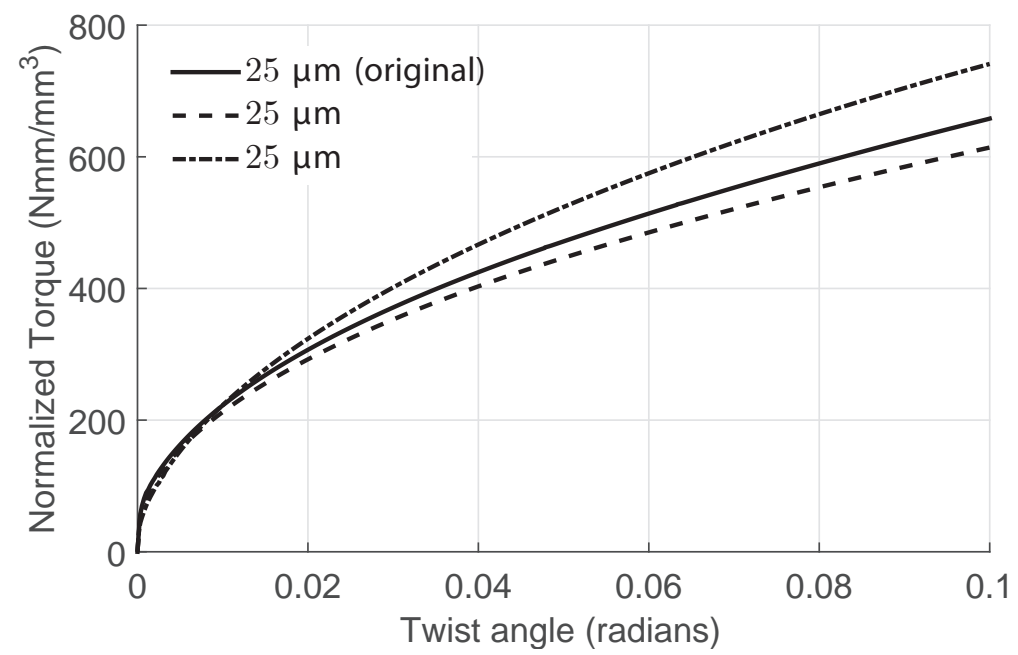

Figure 5.7: Torque vs. twist angle curves with $25 \mu \mathrm{m}$ disc radius and 2 sets of additional randomly generated orientations to show the statistical deviation in the average response.

gradient of slip — exhibits a non-homogeneous distribution. 


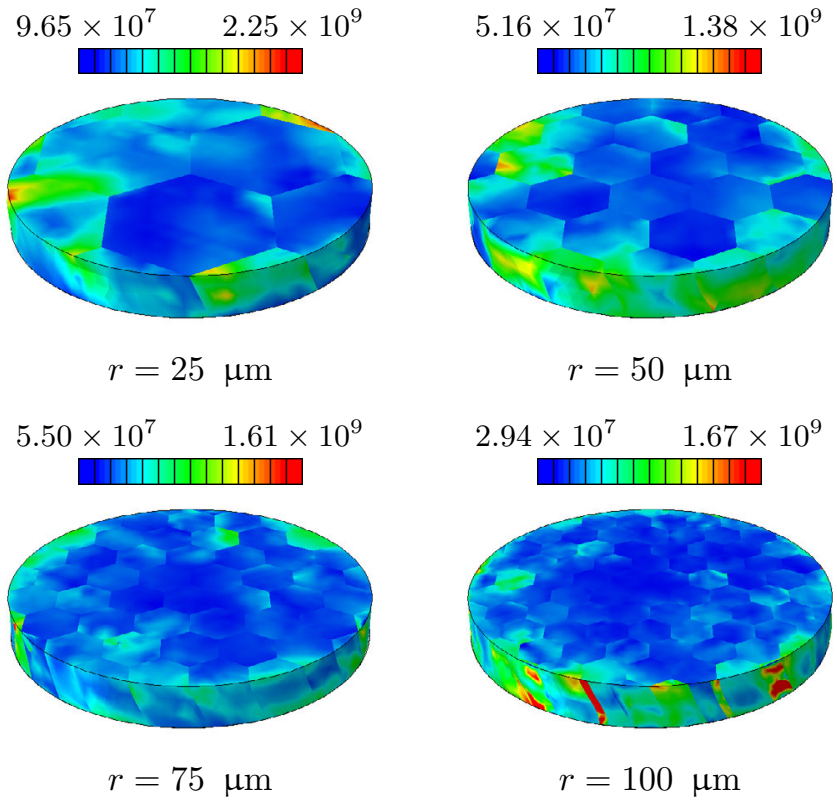

Figure 5.8: Final distribution of GNDs $\left(\mathrm{mm}^{-2}\right)$ in the disc as a result of the applied torsion.

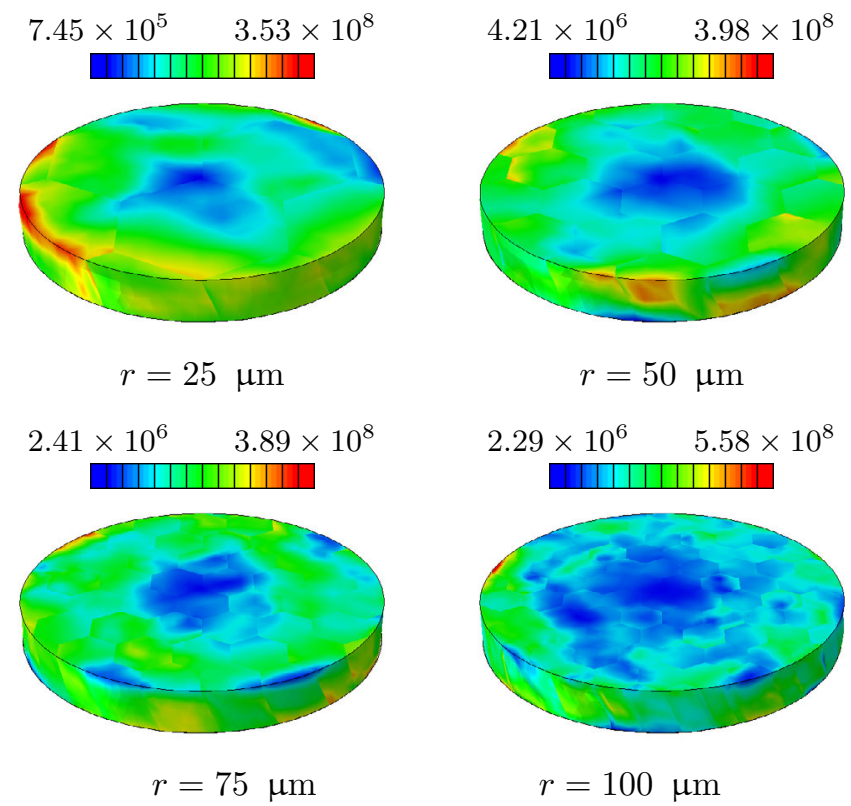

Figure 5.9: Final distribution of SSDs $\left(\mathrm{mm}^{-2}\right)$ in the disc as a result of the applied torsion. 


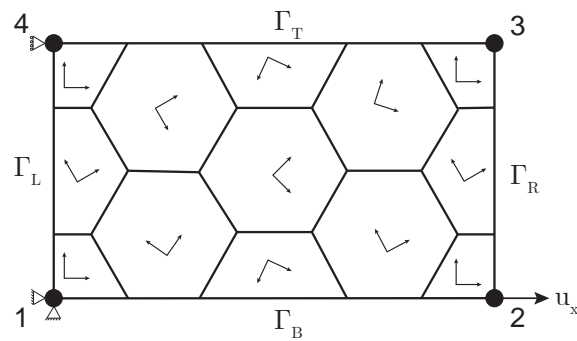

Figure 5.10: Schematic of the applied periodic boundary conditions and the periodicity of the grains. The coordinate axes show lattice orientations

\subsubsection{Grain size strengthening}

The generic geometry, boundary conditions and orientation periodicity are shown in Figure 5.10. Accordingly, in the simulations, all the RVEs are $10 \%$ stretched in the $\mathrm{x}$ direction while periodic boundary conditions are prescribed to the nodes at the edges as given in (5.34).

$$
\mathbf{u}_{\mathrm{T}}-\mathbf{u}_{\mathrm{B}}=\mathbf{u}_{3}-\mathbf{u}_{2} \quad \text { and } \quad \mathbf{u}_{\mathrm{R}}-\mathbf{u}_{\mathrm{L}}=\mathbf{u}_{2}-\mathbf{u}_{1}
$$

Moreover, periodicity of lattice orientations of the grains, which are cut along the edges, were enforced. In this example, four equal-sized RVEs with different grain sizes are tested in order to investigate the phenomenon known as the Hall-Petch effect $[46,147]$. It suggests that with decreasing grain size due to interaction of dislocations with the grain boundaries, the macroscopic yield strength of the structure increases as:

$$
\sigma_{\mathrm{y}}=\sigma_{0}+k \sqrt{d^{-1}}
$$

where $\sigma_{0}$ and $k$ are material parameters.

The grain orientations in RVEs are chosen to give random texture and similar Mackenzie distributions in order to prevent texture related mechanical behavior variations [148]. Details on generated random texture and the list of orientations can be found in the work of Nijhuis [149].

Figure 5.11 shows the generated RVEs and the hexagonal shaped grains. The colors represent grain orientations. As mentioned above, continuity of the edge-grains is ensured. At small grain sizes some colors are repeated due to limited color codes available in software. The RVEs are chosen to have equal size and different number of grains instead of having the same number of grains and varying in size, because classically grain size determination is conducted for a fixed area [150]. In this study, the given grain sizes, $d$, are calculated as the diameter of the circle that fits in the hexagonal grains. 


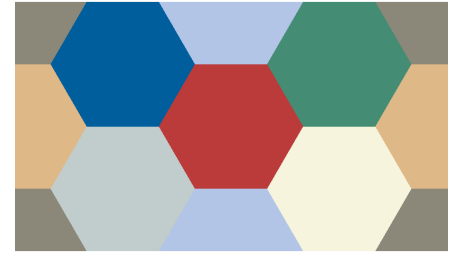

$d=48 \mu \mathrm{m}$

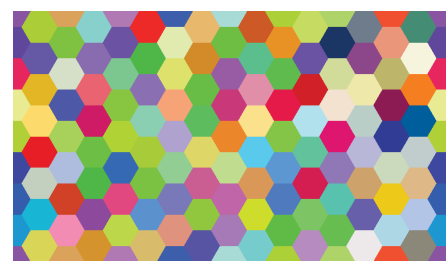

$d=12 \mu \mathrm{m}$

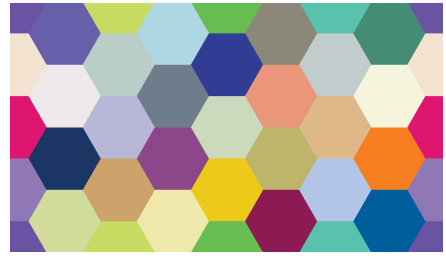

$d=24 \mu \mathrm{m}$

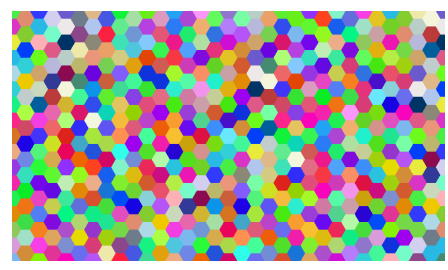

$d=6 \mu \mathrm{m}$

Figure 5.11: Grain size of equally-sized RVEs used in the crystal plasticity simulations. Every crystal (represented by a different color) has a different crystallographic orientation.

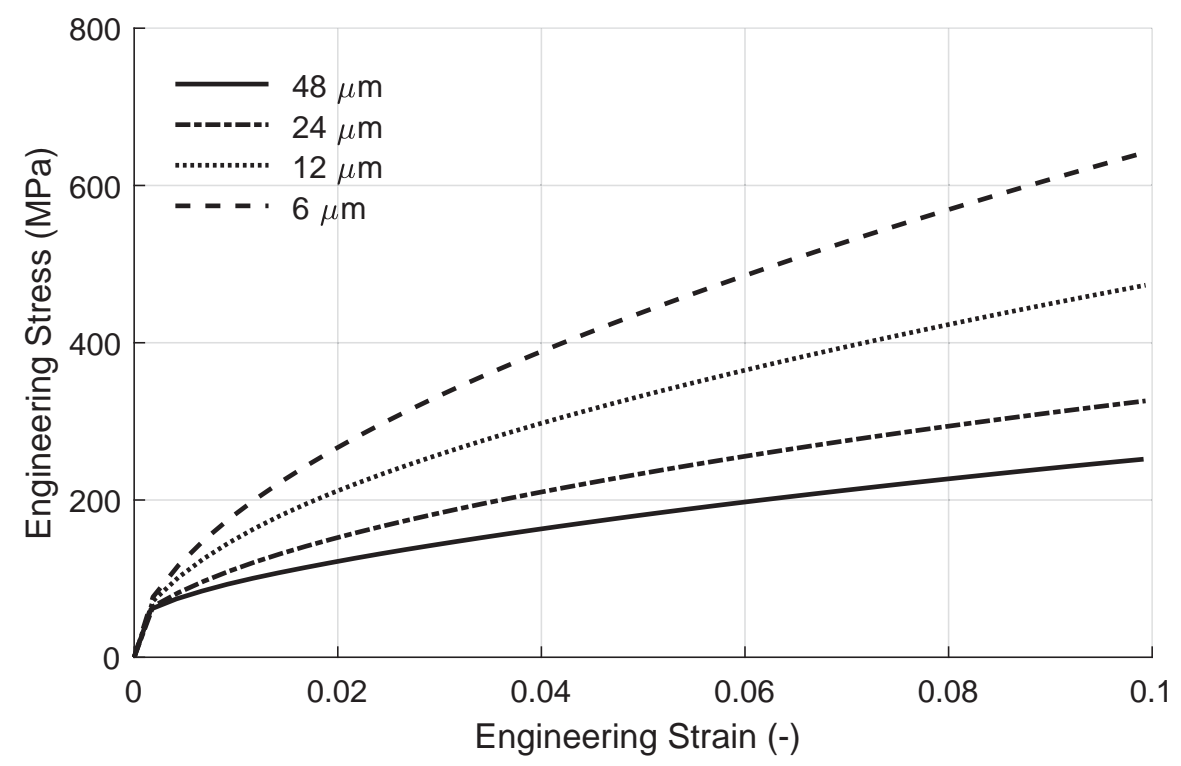

Figure 5.12: Engineering stress-strain curves of the RVEs with different grain sizes.

All the RVEs are meshed with quadratic triangular plane strain elements with three integration points. The same material parameters as used in the torsion example are used. 
The engineering stress-strain curves of the RVEs are shown in Figure 5.12, where a grain size strengthening effect can be seen. Moreover, it is seen that the hardening rate, $\delta \sigma / \delta \epsilon$, also increases with decreasing grain size. As in the torsion examples, the stress strain curves do not show a saturating behavior even if the SSD evolution gives a saturating stress response due to the contribution of GNDs. This is mainly due to the increase of deformation inhomogenity and since the GND evolution does not contain a limit, this increase prevents saturation. The non-saturating type of behavior is also reported in the literature investigating grain size strengthening [47,151-154].

Figure 5.13 shows the change in yield strength with $\mathrm{d}^{-0.5}$ and the linear fit. The yield strengths of the RVEs are measured by employing the well known $0.2 \%$ offset method. This method is frequently used in the literature and industry to calculate the onset of observable plastic deformation. It can be seen that the linear fit describes the change quite well. The linear fit yields a reasonable Hall-Petch coefficient, $k=4.26 \mathrm{MPa} \mathrm{mm}{ }^{0.5}$, which falls in the range of the experimentally determined values $[155,156]$.

Figure 5.14 shows the evolution of average $\rho_{\mathrm{SSD}}$ in the RVEs. The average values are calculated element wise by using first the numerical integration of the three integration points within the element and secondly, weighted average of the element properties with element area. It is seen from the figure that SSD density increases with deformation and the effect of grain size on the evolution is very small. This is due to the fact that the SSDs evolve with strain. However, a slight effect is observed since in each case there is a different grain to grain interaction and there is naturally a variation in plastic strain.

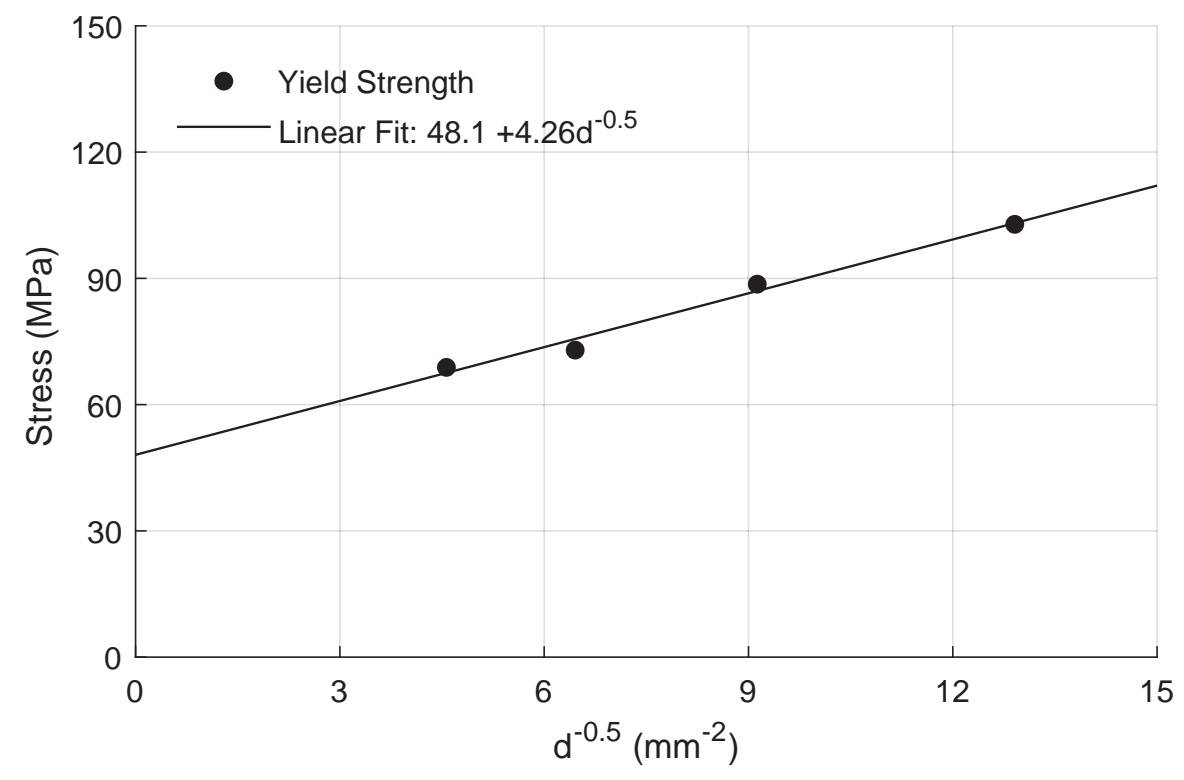

Figure 5.13: Increase of yield strength, calculated by the $0.2 \%$ offset method. 


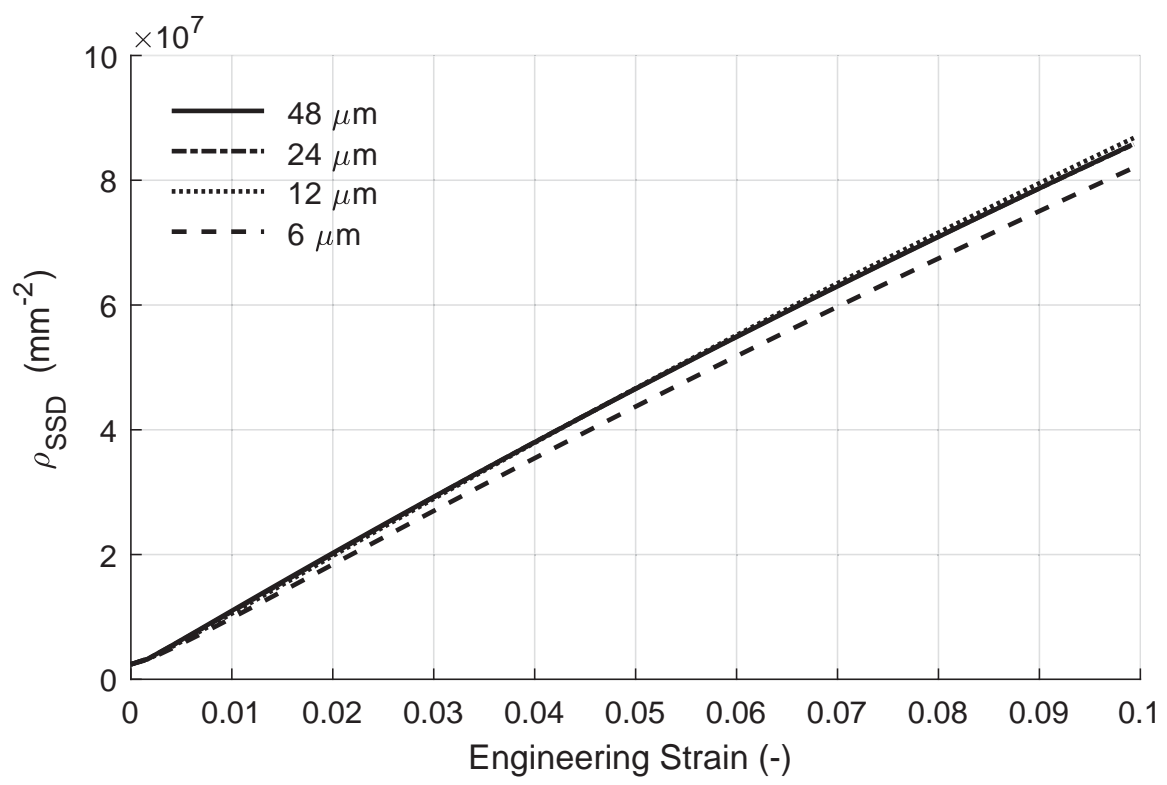

Figure 5.14: Evolution of $\rho_{\mathrm{SSD}}$ with increasing macroscopic engineering strain for equally-sized RVEs with different grain sizes.

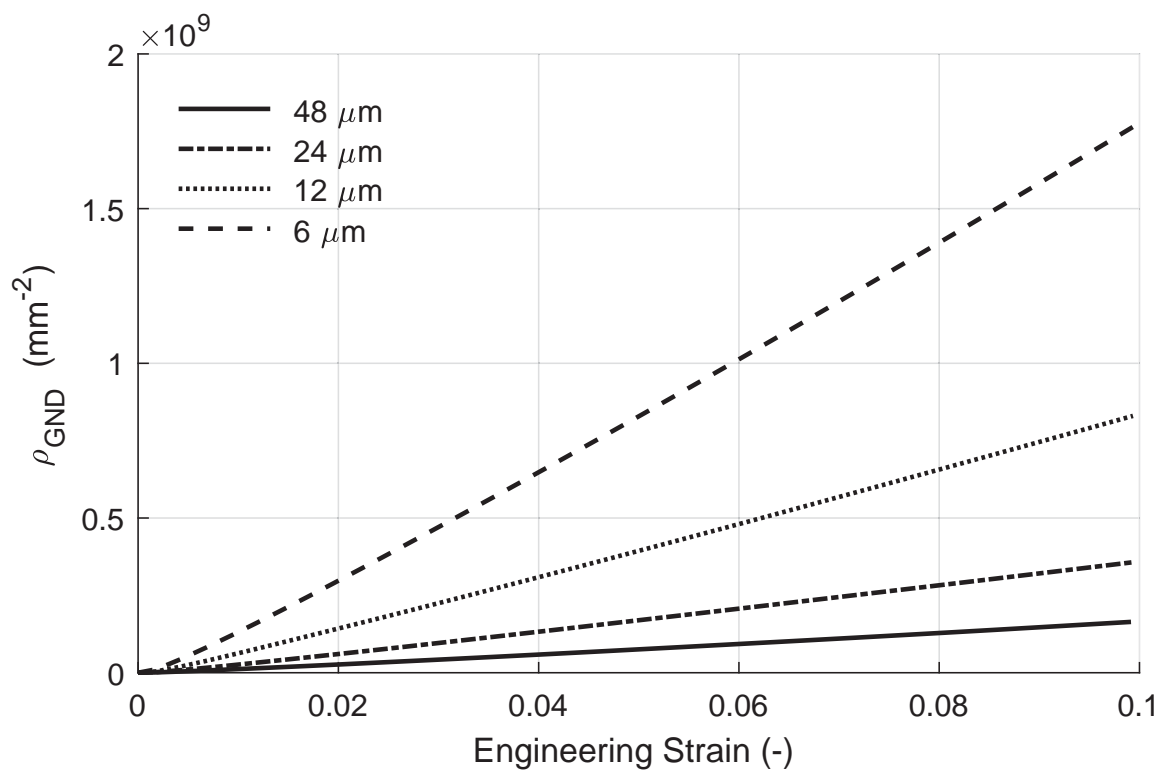

Figure 5.15: Evolution of $\rho_{\mathrm{GND}}$ with increasing macroscopic engineering strain for equally-sized RVEs with different grain sizes. 


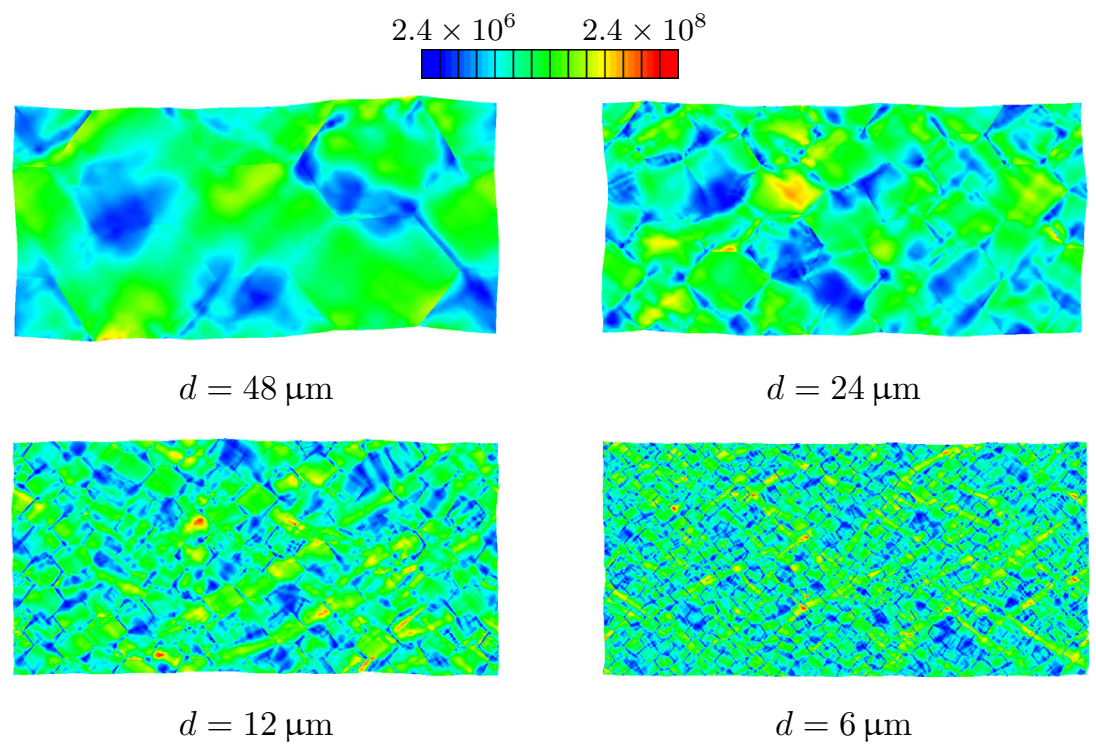

Figure 5.16: Final distribution of SSDs $\left(\mathrm{mm}^{-2}\right)$ in the RVEs after a tensile strain of $10 \%$.

Figure 5.15 shows the evolution of average $\rho_{\mathrm{GND}}$ in the RVEs. The average values are calculated in the same manner as for SSDs. It is seen from the figure that both grain size and applied strain has an effect on the evolution of GNDs. The dependence of GNDs on grain size is two fold. First, with decreasing grain size, the microstructural deformation complexity increases, yielding higher gradients. The second aspect can easily be imagined by considering deformation of the same microstructural morphology but for different sizes. When the size gets smaller the spatial gradient will increase.

Figure 5.16 show the spatial distribution of SSDs in the RVEs at $10 \%$ engineering
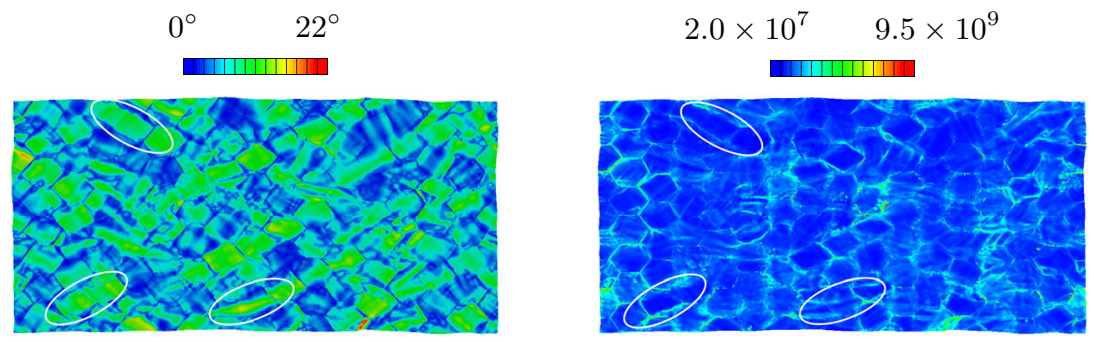

Figure 5.17: Misorientation (with respect to the initial orientation) caused by deformation (left) and GND distribution (right) for one of the RVEs. The white ellipses show the regions where the gradient in the rotation causes GND formation. 


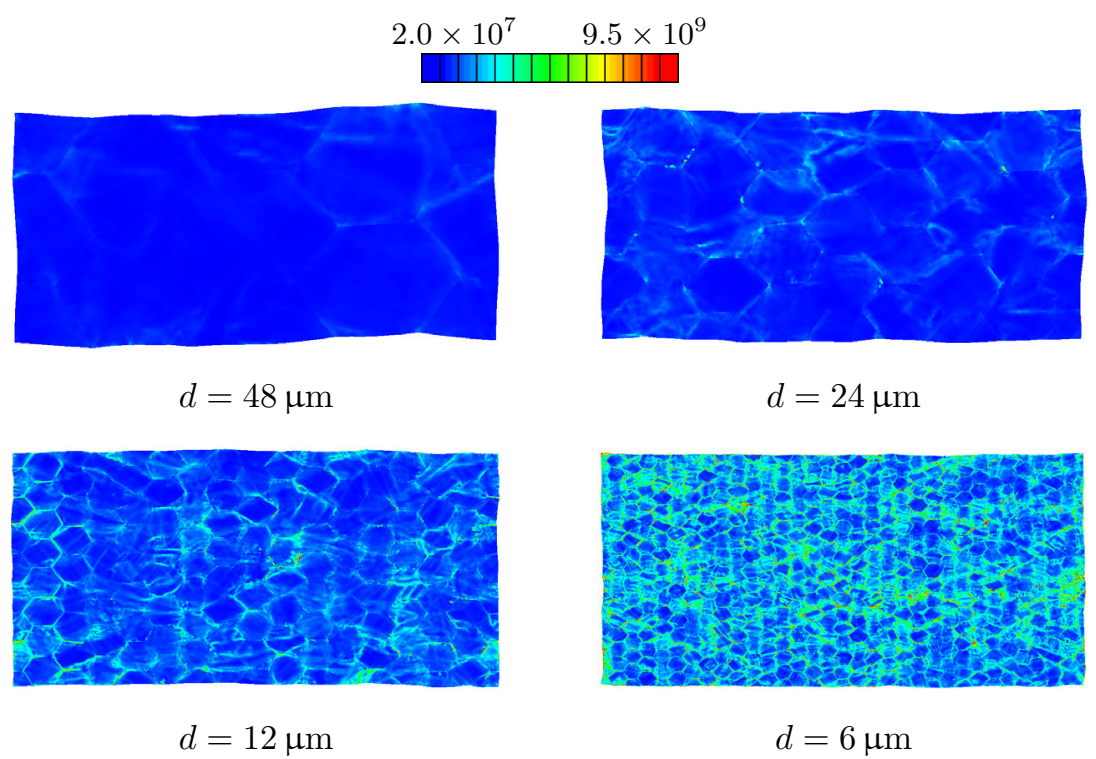

Figure 5.18: Final distribution of GNDs $\left(\mathrm{mm}^{-2}\right)$ in the RVEs after a tensile strain of $10 \%$.

strain. There are some common features that can be observed. The deformation concentrates in shear bands (band shaped plastic deformation zones). Between the shear bands there are areas with low SSD density. With decreasing grain size the thickness of these bands decreases whereas their number and complexity increases. Moreover, with decreasing shear band thickness, the size of the low SSD areas decreases. Another feature of shear bands is that, they naturally create a gradient of strain concentrating at the edges of the shear bands, which will promote GNDs. Since the evolution of local texture is related with plastic deformation, it can be seen that at the locations with a higher gradient in the misorientation (with respect to initial orientation) distribution GNDs are present, see Figure 5.17. The ellipses in that figure, show the location where this can be seen. This is an interesting aspect because local orientation differences between points are frequently used in experimentally measurement of GNDs and the model also captures this behavior.

Figure 5.18 show the spatial distribution of GNDs in the RVEs at $10 \%$ engineering strain. For the large grain size $(d=48 \mu \mathrm{m})$, the number of GNDs is very low. With decreasing grain size the concentration of GNDs increase starting from the grain boundaries, concentrating more at the tripole junctions of grain boundaries. In the smallest grain size it is clearly seen that GNDs are not only concentrated at the grain boundaries but also in the grains, with a very complex distribution. 


\subsection{Conclusions}

In this chapter, a strain gradient enhanced rate-independent crystal plasticity framework was introduced and the details concerning the implementation of the framework for the commercial FE package ABAQUS/STANDARD was given. Moreover, two example problems were introduced showing the general aspects of the model, illustrating the strain gradient effects. It is shown that the explicit gradient approach can capture the strain gradient effects resulting from both geometrical and microstructural conditions.

In the torsion of a disc problem, it is shown that both the structural gradient and the gradient due to lattice orientations play an important role in the normalized torque response of thin wires. Moreover, when the size of the wire becomes small, the statistics of grain orientations start to play an important role.

In the grain size strengthening problem, it is shown that decreasing the grain size causes higher GND densities. On the other hand, SSDs were not effected as much as GNDs. It is shown that the model captures a linear increase in the yield strength with $d^{-0.5}$. It is also shown that decreasing the grain size yields a finer shear band formation and GNDs accumulate at the edges of these shear bands.

Finally, in both simulations the SSD distributions showed that macroscopically prescribed boundary conditions vary locally. 



\section{Damage initiation in dual phase steels}

In this chapter, stress and strain partitioning characteristics and damage initiation in dual phase steels is investigated within the strain gradient enhanced crystal plasticity framework. The effects of martensite distribution (banded or random) and martensite content on damage initiation are investigated by employing RVEs with different characteristics. Different damage indicators, which do not alter the material response, were examined in order to find the most appropriate indicator for ferrite and martensite phases.

\subsection{Introduction}

Dual phase steels are prone to damage initiation due to the mechanical property mismatch between ferrite and martensite. However, the prematurely formed microcracks and voids do not cause a catastrophic brittle failure due to hardening response and localized deformation of ferrite at the crack tips [7]. Nevertheless, the damage initiation and evolution behavior of dual phase steels plays an important role in the formability of this alloy, thus it has been extensively studied.

Numerical investigations on micromechanics of damage initiation and failure are focused on simulating the mechanical response of the alloys with isotropic plasticity models using representative volume elements which are generated by real $[20,157,158]$ or idealized microstructures [159-161]. On the other hand, there are also studies which use crystal plasticity formulation in order to incorporate the effect of plastic anisotropy of the crystals on the stress and strain distribution $[36,37,68]$. The topic of damage initiation is frequently elaborated by using equivalent stress and strain concentrations. Moreover, these studies show consistent results regarding the importance of stress state, predicting failure of the soft phase with a shear dominant mechanism. 
In the recent numerical studies of De Geus et al. [38,39,162,163], a systematic and statistical approach was employed in order to exploit the factors affecting damage initiation in multi-phase materials. The authors focused mainly the investigations on important factors such as the contrast between the mechanical properties of the phases, crystal orientations and loading conditions. It was shown in their works that, after a threshold, void nucleation occurs throughout the deformation. The investigations into the effect of average stress triaxiality values in the range of 0 to 1 showed that damage initiation in the soft phase was promoted by low values while the high triaxiality increased damage initiation in the hard phase. They have predicted a higher damage initiation rate with increasing $2^{\text {nd }}$ phase fraction or mechanical property mismatch. Moreover, they have identified that localization was controlled by alignment of several voids in a shear configuration and the instability point was strongly affected by the configuration of the voids, resulting in a large scatter in fracture strain.

In the present chapter, we investigate the effect of microstructural factors on stress and strain partitioning and damage initiation probability in dual phase steel by finite element analysis. The main objective is to identify damage prone features in DP steel microstructures which may cause damage initiation and premature failure. We focus our investigation on the microstructural features which can be controlled in the production stage of dual phase steel sheets. For this purpose 2D artificial RVEs are generated with different martensite contents (9, 18 and 27\%) and martensite distributions (banded vs. random). The RVEs are deformed uniaxially under periodic boundary conditions while a strain gradient enhanced crystal plasticity framework is used to model material behavior. Damage initiation is investigated by using several damage indicators which are uncoupled and therefore do not influence the material behavior. These damage indicators are compared with each other and the location of damage incidents that were observed in Chapter 4, in order to find out the suitable indicator with predictive capabilities.

\subsection{Microstructures and material properties}

Four dual phase RVEs with different microstructural characteristics were generated to analyze the effect of microstructure on damage initiation. The investigated microstructural features were based on the variations that exist between different grades of dual phase steels or the same grade with different processing parameters. These main microstructural features are distribution of martensite and martensite phase fraction.

The artificial 2D-RVEs with a size of $75 \times 75 \mu \mathrm{m}$ were generated via the Microstructure Design tool: Multilevel Voronoi (MLV) tessellation software developed by Tata Steel. The working principle of the software is summarized briefly in Chapter 7. Figure 6.1 shows the generated RVEs. Ferrite (left) and martensite (right) phases were illustrated separately and colored according to lattice orientations. The grain orientations of the RVEs were generated randomly for both phases. Table 6.1 gives a quantitative summary of the features such as 
Table 6.1: Characteristics of the generated RVEs. Subscripts F and M indicate ferrite and martensite.

\begin{tabular}{cccccc}
\hline & $\begin{array}{c}\text { Area } \\
(\%)\end{array}$ & $\begin{array}{c}\mathrm{d}_{\mathrm{F}} \\
(\mu \mathrm{m})\end{array}$ & $\begin{array}{c}\mathrm{d}_{\mathrm{M}} \\
(\mu \mathrm{m})\end{array}$ & $\begin{array}{c}\text { \# of Grains } \\
(-)\end{array}$ & $\begin{array}{c}\text { \# of Grains } \\
(-)\end{array}$ \\
\hline Banded & 18 & 5.4 & 4.1 & 197 & 77 \\
Random & 9 & 5.8 & 3.0 & 197 & 71 \\
Random & 18 & 5.4 & 3.9 & 197 & 89 \\
Random & 27 & 5.1 & 4.4 & 198 & 102 \\
\hline
\end{tabular}

martensite fraction, average ferrite and martensite grain diameter, number of ferrite and martensite grains. The RVEs can be grouped into two categories according to the distribution of martensite (random or banded) and martensite fraction (9-18-27\%). The results and discussions will follow this grouping.

The material properties that are used in the simulations are given in Table 6.2 to represent ferrite-like and martensite-like behavior. Both phases were assumed to have a BCC structure and deform only by slip on the $\langle 111\rangle\{110\}$ family. The contribution of tension-compression asymmetry and non-Schmid effects to the deformation was discussed in Chapter 5 and its effect was given in Eq. (5.15). The three non-Schmid coefficients are taken from the experimental fitting from the research carried out by Patra et al. [123]. For the latent hardening of slip systems, the physically based interaction matrix $Q^{(\alpha \beta)}$ was employed with the six independent coefficients, given in Table 5.1 [135]. The initial dislocation densities for each slip system of each phase was calculated from the total dislocation density that is reported in literature as $9.0 \times 10^{7}$ $\mathrm{mm}^{-2}$ for ferrite [164] and $1.6 \times 10^{9} \mathrm{~mm}^{-2}$ for martensite [165]. These densities were assumed to be distributed equally over the 12 possible slip systems yielding dislocation densities of $7.50 \times 10^{6} \mathrm{~mm}^{-2}$ and $1.34 \times 10^{8} \mathrm{~mm}^{-2}$ for each slip system of ferrite and martensite, respectively. The RVEs were meshed with $2^{\text {nd }}$ order, triangular, plane strain elements (CPE6M) and deformed 10\% under periodic boundary conditions.

\subsection{Results and discussion}

\subsubsection{Partitioning of stress and strain}

The average true stress-strain response of the RVEs (solid lines) and the average stress and strain partitioning between ferrite (dotted line) and martensite (dashed line) are given in Figure 6.2. The average stress and strain values of the RVEs were calculated from the reaction force along the loading direction. On the other hand, average stressstrain of ferrite and martensite phases are calculated by using a weighted average of the elemental stress and strain values. 
(a)

(b)

(c)

(d)
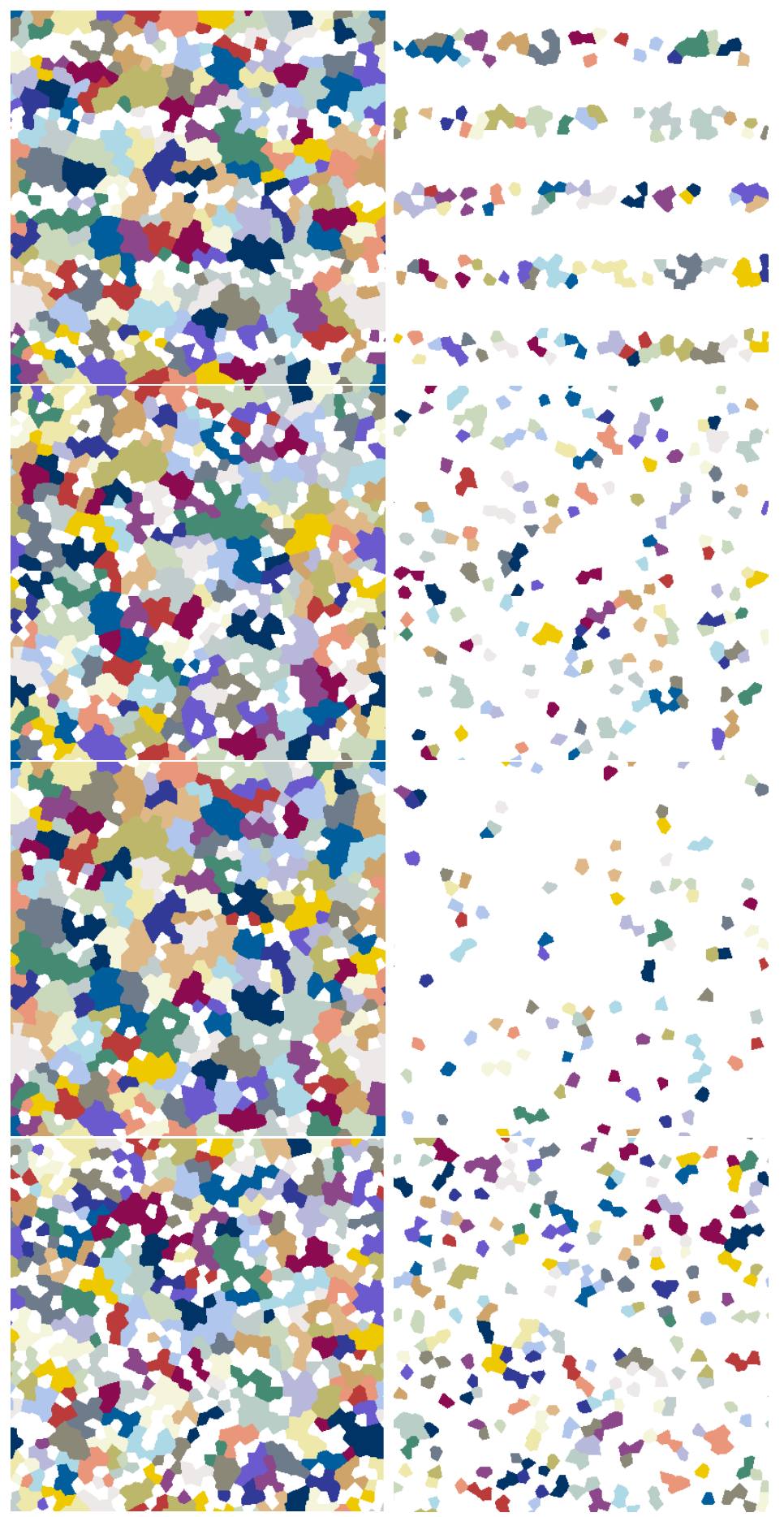

Figure 6.1: Generated artificial RVEs. Left: Ferrite, Right: Martensite. (a)Banded $18 \%$ martensite, (b)Random - 18\% martensite, (c)Random - 9\% martensite, (d)Random - 27\% martensite. Colors are to differentiate different grains. 
Table 6.2: Material parameters for ferrite and martensite

\begin{tabular}{ccc}
\hline & Ferrite & Martensite \\
\hline $\mathrm{E}(\mathrm{GPa})$ & 212 & 212 \\
$\nu(-)$ & 0.3 & 0.3 \\
$\tau_{0}(\mathrm{MPa})$ & 40 & 250 \\
$b(\mathrm{~mm})$ & $2.48 \times 10^{-7}$ & $2.48 \times 10^{-7}$ \\
$\rho_{0}\left(\mathrm{~mm}^{-2}\right)$ & $7.50 \times 10^{6}$ & $1.34 \times 10^{8}$ \\
$\rho^{\infty}\left(\mathrm{mm}^{-2}\right)$ & $5.00 \times 10^{8}$ & $5.00 \times 10^{8}$ \\
$\gamma^{\infty}(-)$ & 0.4 & 0.3 \\
\hline
\end{tabular}

When the average true stress-strain curves are compared, it is seen that the strength of RVEs is more dependent on the martensite content than on the distribution of martensite. With increasing fraction of martensite both flow stress and rate of hardening $\left(\mathrm{d} \sigma_{11} / \mathrm{d} \varepsilon_{11}\right)$ increase. On the other hand, the effect of martensite morphology is more pronounced on the initial hardening rate than on the flow stress. The initial hardening rate is higher for the banded morphology but it reduces more rapidly than the random morphology, so the tensile stress response of the random structure catches up to the banded structure.

Moreover, we can compare how stress and strain are partitioned and how this partitioning is effected from the $2^{\text {nd }}$ phase amount and distribution by using the dotted and dashed lines from Figure 6.2. It is seen that there is no unique average behavior for ferrite and martensite. One reason for that can be attributed to the grain size differences between the RVEs. However, it cannot be the main reason since this slight difference is not expected to yield as much variation of the average hardening response. Even more, when the grain size of martensite in the RVEs is compared with the hardening response, it is seen that the hardening is actually in the opposite trend of what is expected. Therefore, the main factor causing the variation in the hardening response of the phases in the RVEs should be the interactions (e.g., increased GND content and nonuniform deformation at the interface) between phases. In the study of Ghassemi-Armaki et al. [6], it was shown experimentally that the ferrite phase closer to martensite islands are harder. This suggests that the average behavior of a phase would be affected by the distribution of the other phase(s). A schematic illustration is given in Figure 6.3 to show the effect of $2^{\text {nd }}$ phase distribution and fraction on the stress and strain partitioning characteristics. The main effect of $2^{\text {nd }}$ phase distribution is on the slope of the tie line joining stress and strain states of the phases. The banded morphology exhibits a more iso-strain like behavior, as expected. In addition, the martensite in the banded distribution shows a higher strain hardening rate. On the other hand, the effect of the martensite fraction has very little influence on the slope of the tie line. The effect is more pronounced on the stress-strain curves of the partitioning phases. In general, the trend with increasing martensite content is that the strain hardening rate of both phases increases. 


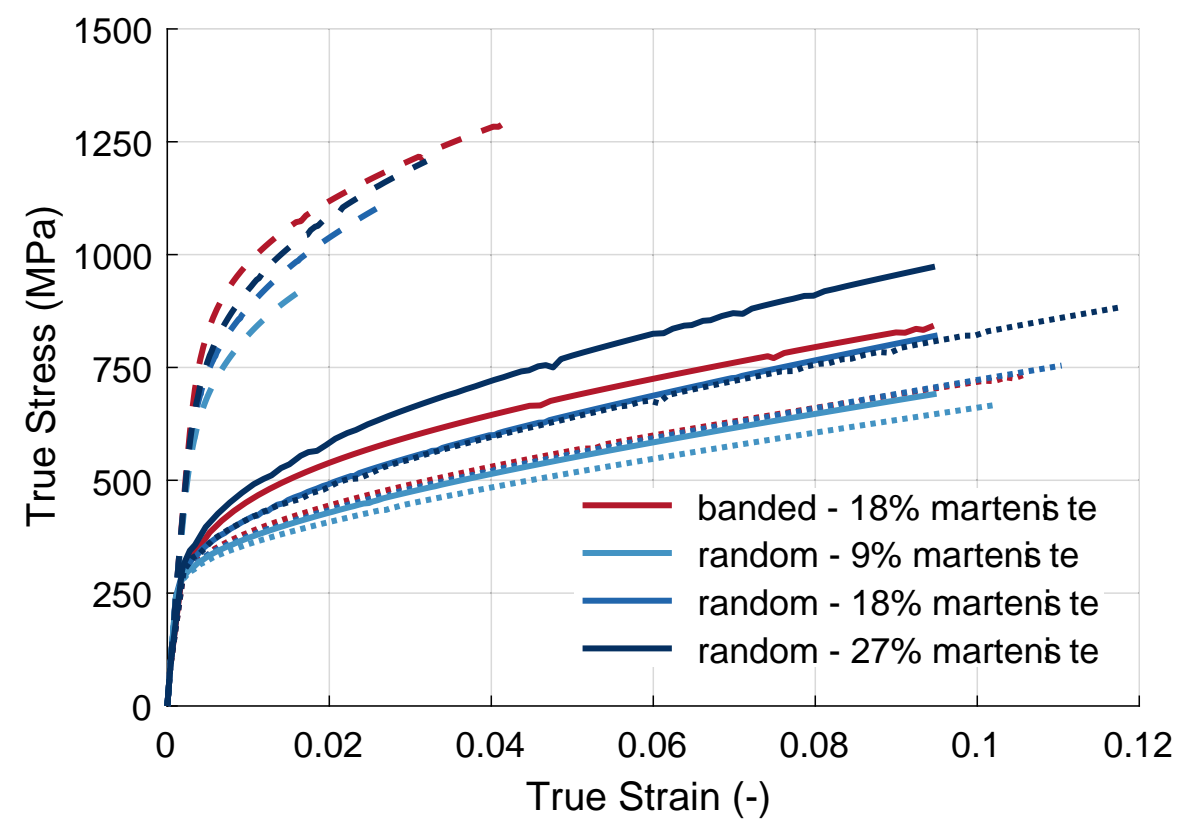

Figure 6.2: True stress and strain response of the RVEs. Dashed lines show average martensite response and dotted lines show average ferrite response of the associated color.
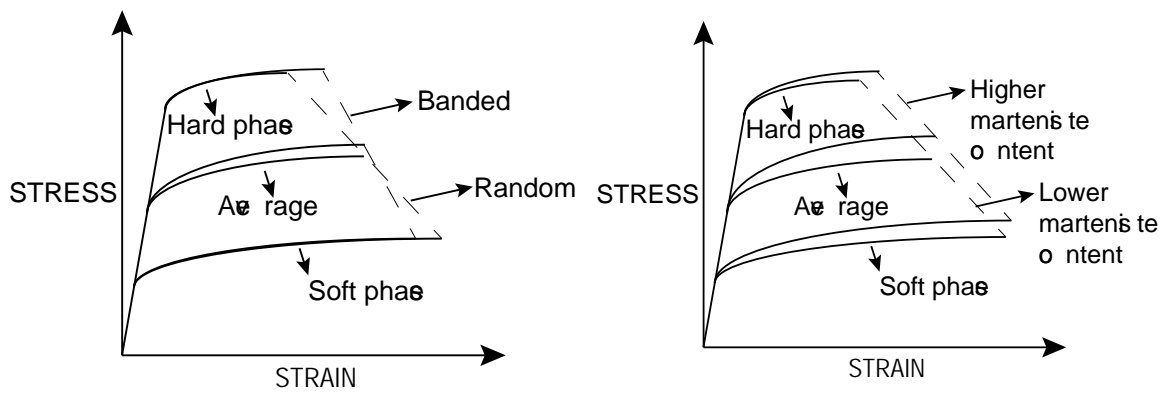

Figure 6.3: Schematic illustration of effect of $2^{\text {nd }}$ phase morphology (left) and fraction (right) on the stress and strain partitioning characteristics.

\subsubsection{Comparison of damage indicators for martensite}

In this section, three different damage indicators $\left(\mathrm{D}^{\mathrm{m}}\right)$ are compared for the martensite phase. One of these indicators is stress-based while the other two are plastic work based criteria. Stress based criteria are commonly used to predict cracking of brittle materials. Martensite is more brittle compared to ferrite; however, it is known that martensite in dual phase steels exhibits plastic deformation $[6,85,166]$. Therefore, we can assume damage would initiate between the stress-based (lower limit) criterion 
and the plastic work-based (upper limit) criterion.

The damage indicators that are used for martensite are given in Equations (6.1), (6.2) and (6.3) and they were calculated from the averaged values (e.g., stress, strain and slip rate) of the finite elements. Moreover, we use absolute values of the indicators, instead of normalized value of the indicators by a critical damage value since the indicators will be compared in terms of their characteristics of localization in the microstructures.

The first damage indicator for martensite, $\mathrm{D}_{1}^{\mathrm{m}}$, is the maximum principal stress, $\sigma_{1}$;

$$
\mathrm{D}_{1}^{\mathrm{m}}=\sigma_{1}
$$

The second damage indicator for martensite, $\mathrm{D}_{2}^{\mathrm{m}}$, is the plastic work-based on the Von Mises yield criterion and it is calculated as:

$$
\mathrm{D}_{2}^{\mathrm{m}}=\int_{0}^{t} \bar{\sigma} \dot{\bar{\epsilon}}^{\mathrm{p}} \mathrm{d} t^{\prime}
$$

The third damage indicator for martensite, $\mathrm{D}_{3}^{\mathrm{m}}$, is plastic work-based on the resolved shear stress and slip amount of the active slip systems and it is in the form of

$$
\mathrm{D}_{3}^{\mathrm{m}}=\int_{0}^{t} \sum_{\alpha}\left[\boldsymbol{\sigma}:\left(\mathbf{s}^{(\alpha)} \otimes \mathbf{m}^{(\alpha)}\right)\right] \dot{\gamma}^{(\alpha)} \mathrm{d} t^{\prime}
$$

where $\mathbf{s}^{(\alpha)}$ is the slip direction, $\mathbf{m}^{(\alpha)}$ is the normal of slip plane and $\dot{\gamma}^{(\alpha)}$ is slip increment of the system $\alpha$.

The damage indicators $\mathrm{D}_{2}^{\mathrm{m}}$ and $\mathrm{D}_{3}^{\mathrm{m}}$ are similar but $\mathrm{D}_{2}^{\mathrm{m}}$ is derived for a material based on a Von Mises type yield locus and represents a more averaged work. On the other hand, $\mathrm{D}_{3}^{\mathrm{m}}$ is derived consistent with the underlying crystal plasticity approach in which a yield function does not exist but individual slip systems have critical values above which deformation takes place.

Figures 6.4 to 6.6 show the distribution of the damage indicators for the banded RVE. In Figure 6.4, it is seen that the damage indicator $\mathrm{D}_{1}^{\mathrm{m}}$ exhibits a more diffuse distribution in the martensite than the other two indicators. It concentrates mainly at the sharp corners of martensite islands closer to the ferrite phase. The indicator hardly forms any hot spots that cuts or breaks through the martensite island, which was frequently observed in the experimental results, Figure 4.8 . Thus, $\mathrm{D}_{1}^{\mathrm{m}}$ is not a very suitable damage indicator.

Figures 6.5 and 6.6 show the plastic work-based damage indicators and they show similar characteristics but $\mathrm{D}_{3}^{\mathrm{m}}$ is more localized at some spots. Both indicators concentrate at elements close to ferrite, at the narrow parts of martensite islands. Moreover, they also form hot spots that form crack like patterns as in Figure 4.8. With these aspects both indicators seems to be able to locate possible sites better than $\mathrm{D}_{1}^{\mathrm{m}}$. Both $\mathrm{D}_{2}^{\mathrm{m}}$ and $\mathrm{D}_{3}^{\mathrm{m}}$ show similar characteristics, which was expected.

Figure 6.7 shows the intensity distribution of the two damage indicators, $\mathrm{D}_{1}^{\mathrm{m}}$ and $\mathrm{D}_{3}^{\mathrm{m}}$ for four different RVEs. $D_{2}^{m}$ is not plotted since it gave very similar results compared to 


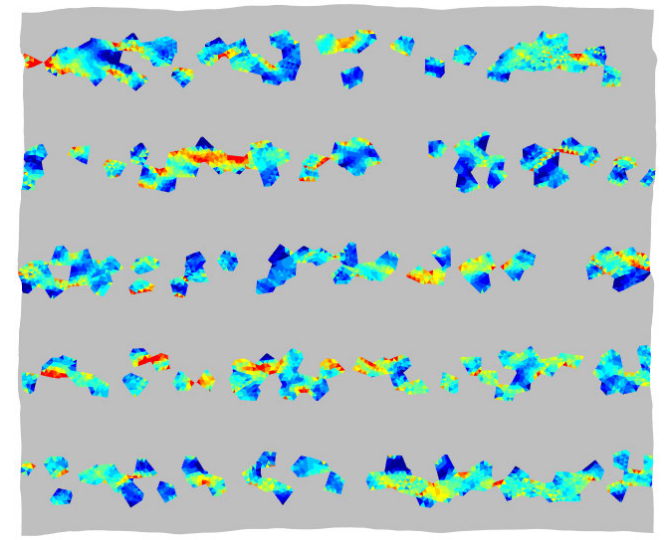

Figure 6.4: Distribution of $D_{1}^{m}$ for the banded RVE.

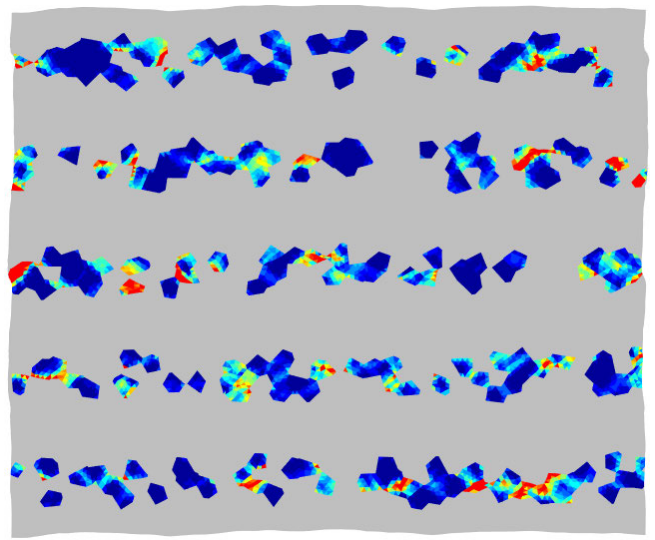

Figure 6.5: Distribution of $D_{2}^{m}$ for the banded RVE.

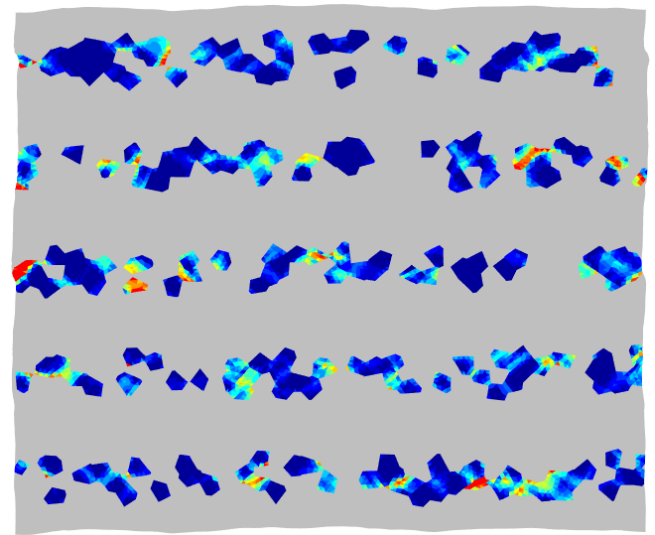

Figure 6.6: Distribution of $\mathrm{D}_{3}^{\mathrm{m}}$ for the banded RVE. 
(a)

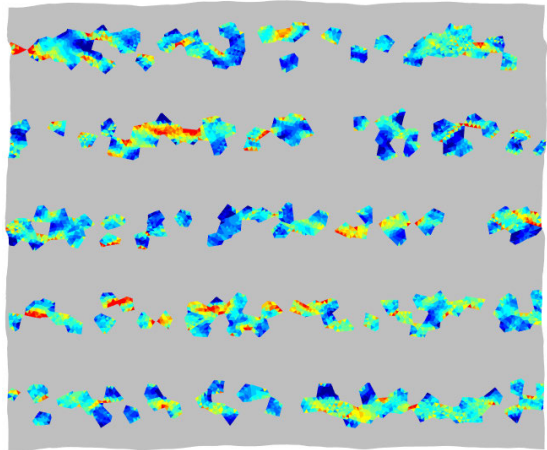

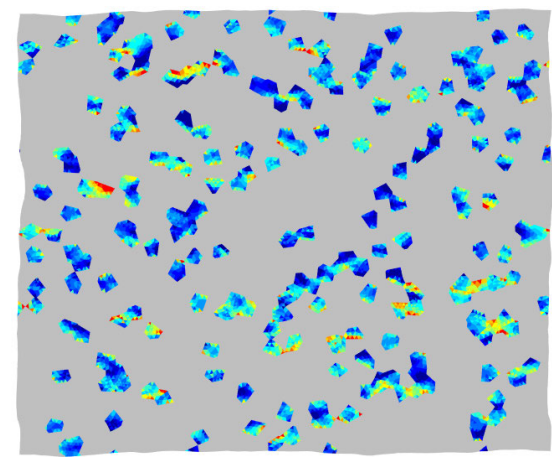

(c)

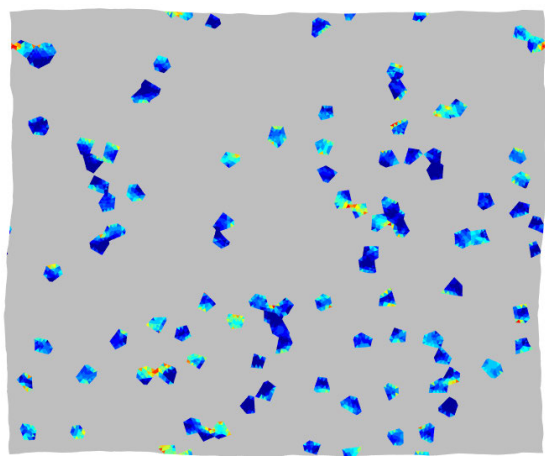

(d)

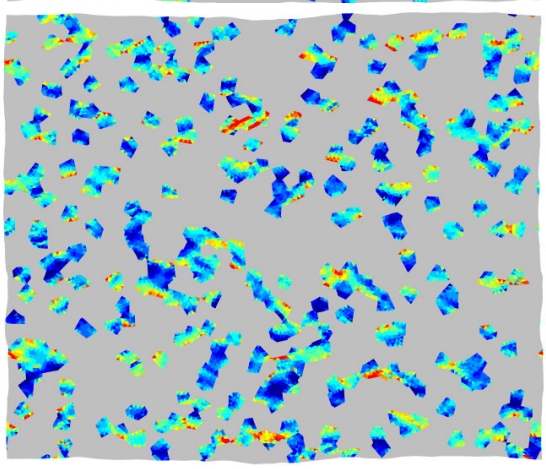

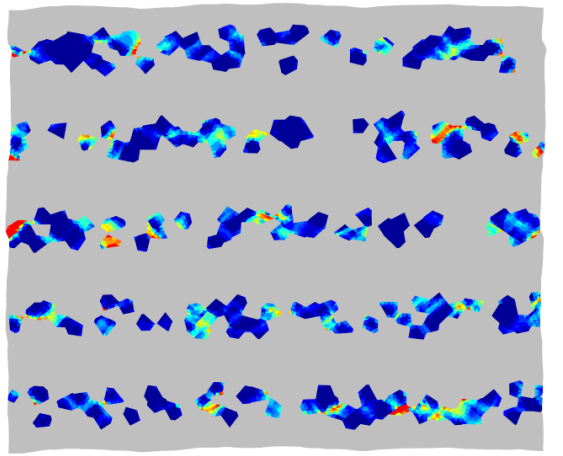
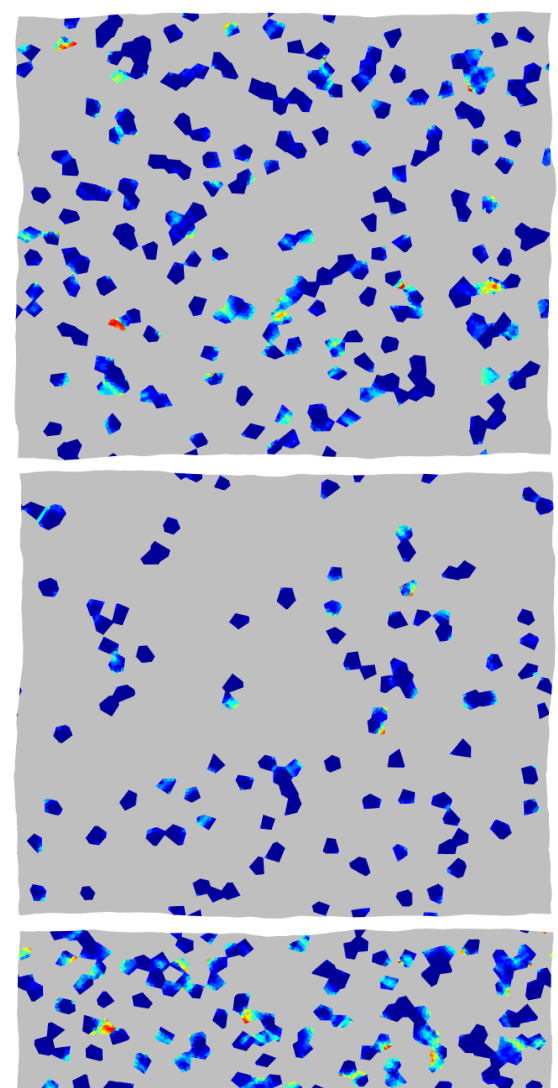

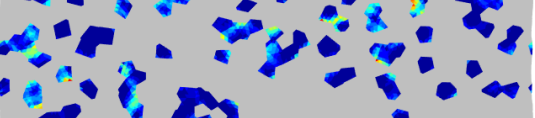

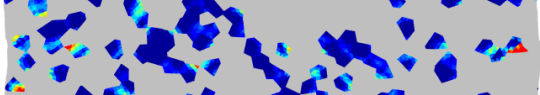

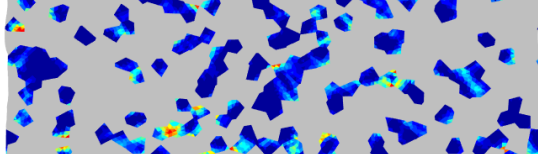

Figure 6.7: Distribution of damage indicators. Left: $D_{1}^{m}$, Right: $D_{3}^{m}$. (a)Banded 18\% martensite, (b)Random - 18\% martensite, (c)Random - 9\% martensite, (d)Random - 27\% martensite. 

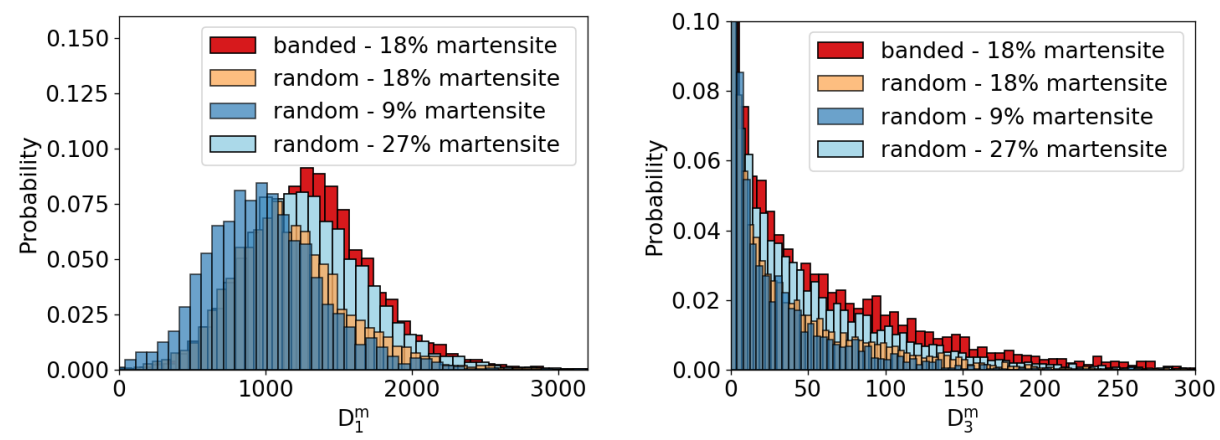

Figure 6.8: Histograms of Left: $\mathrm{D}_{1}^{\mathrm{m}}$ and Right: $\mathrm{D}_{3}^{\mathrm{m}}$.

$\mathrm{D}_{3}^{\mathrm{m}}$. It can be seen from Figures $6.7(\mathrm{a})$ and (b) that change of martensite morphology from banded to random decreases the intensity of both of the damage indicators of martensite, which suggests a reduction in the damage probability of martensite. The increase in the martensite content, on the other hand, increases the intensity of the indicators.

Furthermore, we can compare the distribution of the damage indicator intensities in the RVEs in order to predict which microstructural features (martensite morphology and content) promote martensite damage by using the histograms in Figure 6.8. Since the indicators are not calibrated or normalized, we cannot quantify exactly the microscopic amount of damage. Nevertheless, an increase in the absolute value of the indicators means a higher damage initiation probability. From this perspective,the indicators show similar trends. It can be seen from the histograms of $\mathrm{D}_{1}^{\mathrm{m}}$ and $\mathrm{D}_{3}^{\mathrm{m}}$ that with an increasing percentage of martensite the distributions exhibit higher values suggesting a higher damage probability in the martensite phase. In addition to that for the banded martensite morphology, the histograms contains the highest values. Consequently, we can say the banded structure is the more prone to damage in martensite than their random morphology.

\subsubsection{Comparison of damage indicators for ferrite}

In this section, four different damage indicators $\left(\mathrm{D}^{\mathrm{f}}\right)$ are compared for the ferrite. One of these indicators is strain-based, and two are plastic work-based criteria, which were also used for martensite and the last one is a GND density-based criterion. The strainbased criterion is used to predict failure in ductile materials and is frequently employed for ferrite in the literature $[20,157]$. Moreover, we propose a new criterion based on GND density evolution and hydrostatic stress. In strain gradient models, GND density evolves in order to close the microstructural and rotational incompatibility. While closing the incompatibility, it is assumed that the material stays compatible by generation of GNDs meaning that no gaps form in the microstructure. Based on this idea, we propose that a higher GND density in combination with hydrostatic stress in the numerical model may be used as a sign of void nucleation. 
The damage indicators that are used for ferrite are given in Equations (6.4) to (6.7). They were calculated from the averaged quantities of the finite elements as in the case for the indicators of martensite. Again, we use the absolute (non-normalized) values of the indicators in order to investigate the effect of morphological features (martensite distribution and percentage) on damage probability in ferrite.

The first damage indicator for ferrite, $\mathrm{D}_{1}^{\mathrm{f}}$, is the sum of the slip rate $\dot{\gamma}^{(\alpha)}$ for the slip system $(\alpha)$ :

$$
\mathrm{D}_{1}^{\mathrm{f}}=\int_{0}^{t} \sum_{\alpha} \dot{\gamma}^{(\alpha)} \mathrm{d} t^{\prime}
$$

which is equivalent to integrating effective plastic strain rate.

The second damage indicator for ferrite, $\mathrm{D}_{2}^{\mathrm{f}}$, is the plastic work based on the Von Mises yield criterion and is calculated as:

$$
\mathrm{D}_{2}^{\mathrm{f}}=\int_{0}^{t} \bar{\sigma} \dot{\bar{\epsilon}}^{\mathrm{p}} \mathrm{d} t^{\prime}
$$

The third damage indicator for ferrite, $\mathrm{D}_{3}^{\mathrm{f}}$, is the plastic work based on the resolved shear stress and slip amount of active slip systems and is in the form of

$$
\mathrm{D}_{3}^{\mathrm{f}}=\int_{0}^{t} \sum_{\alpha}\left[\boldsymbol{\sigma}:\left(\mathbf{s}^{(\alpha)} \otimes \mathbf{m}^{(\alpha)}\right)\right] \dot{\gamma}^{(\alpha)} \mathrm{d} t^{\prime}
$$

The last damage indicator for ferrite, $\mathrm{D}_{4}^{\mathrm{f}}$, is calculated as:

$$
\mathrm{D}_{4}^{\mathrm{f}}=\int_{0}^{t} \sum_{\alpha} \sigma_{\mathrm{m}} \dot{\rho}_{\mathrm{GND}}^{(\alpha)} \mathrm{d} t^{\prime}
$$

where $\sigma_{\mathrm{m}}$ is the mean stress (average of the normal stress components) and $\dot{\rho}_{\mathrm{GND}}^{(\alpha)}$ is the rate of geometrically necessary dislocation density of system $\alpha$.

Figures 6.9 to 6.12 show the distribution of damage indicators for the banded RVE. It can be seen that all the damage indicators for ferrite give hot spots next to martensite islands and in the narrow channels between martensite islands. These locations were consistent with the experimentally observed locations, especially for the indicators $\mathrm{D}_{2}^{\mathrm{f}}$ and $\mathrm{D}_{3}^{\mathrm{f}}$. The three indicators, $\mathrm{D}_{1}^{\mathrm{f}}, \mathrm{D}_{2}^{\mathrm{f}}$ and $\mathrm{D}_{3}^{\mathrm{f}}$, show very similar trends. On the other hand, $\mathrm{D}_{4}^{\mathrm{f}}$ shows very localized regions of hot spots which separates it from the other indicators.

Furthermore, we can compare qualitatively the change in the damage prone features in the RVEs by using histograms given in Figure 6.13. As mentioned before, since the indicators are not calibrated, we cannot quantify exactly the microscopic damage amount. However, an increase in the high values of the indicator or an extension of the right hand side tail suggest a higher damage initiation probability. In this aspect, all the indicators show that the highest damage probability in ferrite is for the random structure with 27 vol\% martensite content and lowest when the martensite content 


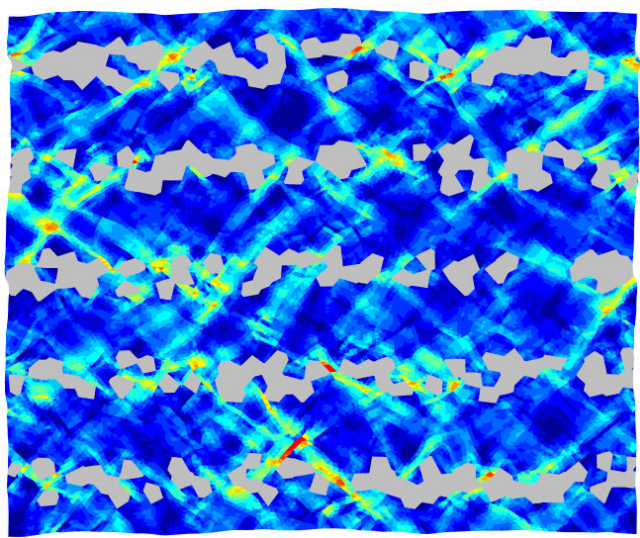

Figure 6.9: Distribution of $\mathrm{D}_{1}^{\mathrm{f}}$ for the banded RVE.

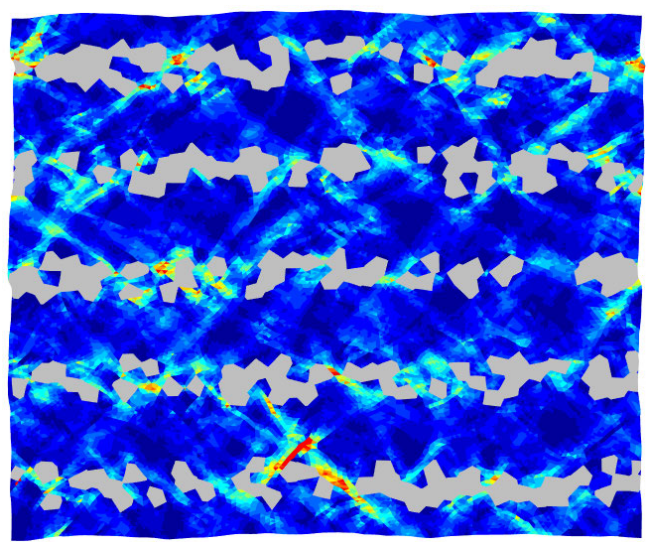

Figure 6.10: Distribution of $\mathrm{D}_{2}^{\mathrm{f}}$ for the banded RVE.

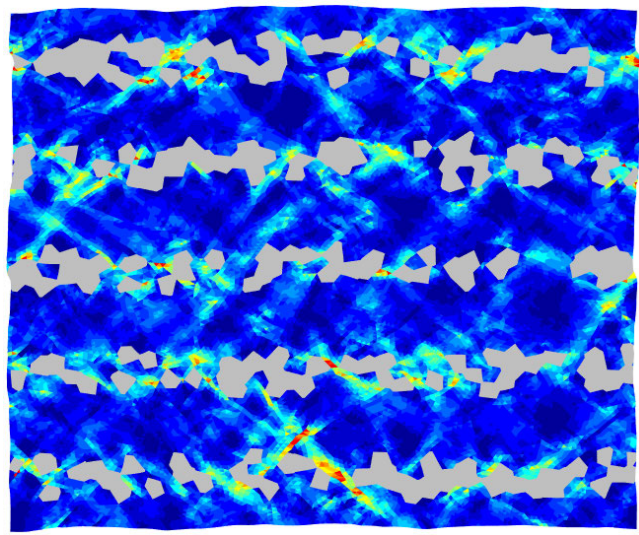

Figure 6.11: Distribution of $\mathrm{D}_{3}^{\mathrm{f}}$ for the banded RVE. 


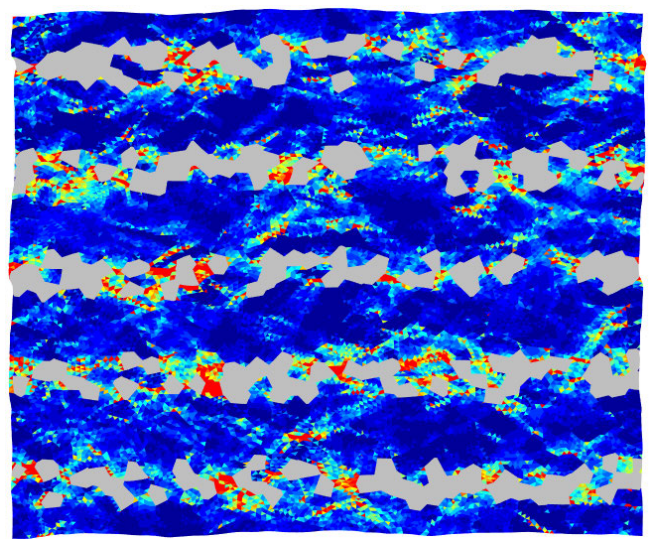

Figure 6.12: Distribution of $\mathrm{D}_{4}^{\mathrm{f}}$ for the banded RVE.

is the lowest. This is also in agreement with partitioning of stress and strain in the RVEs, see Figure 6.2. In that figure, it was shown that increasing martensite content results in higher average stress and strain values in ferrite. When we compare the indicator distributions for different martensite morphologies with the same content
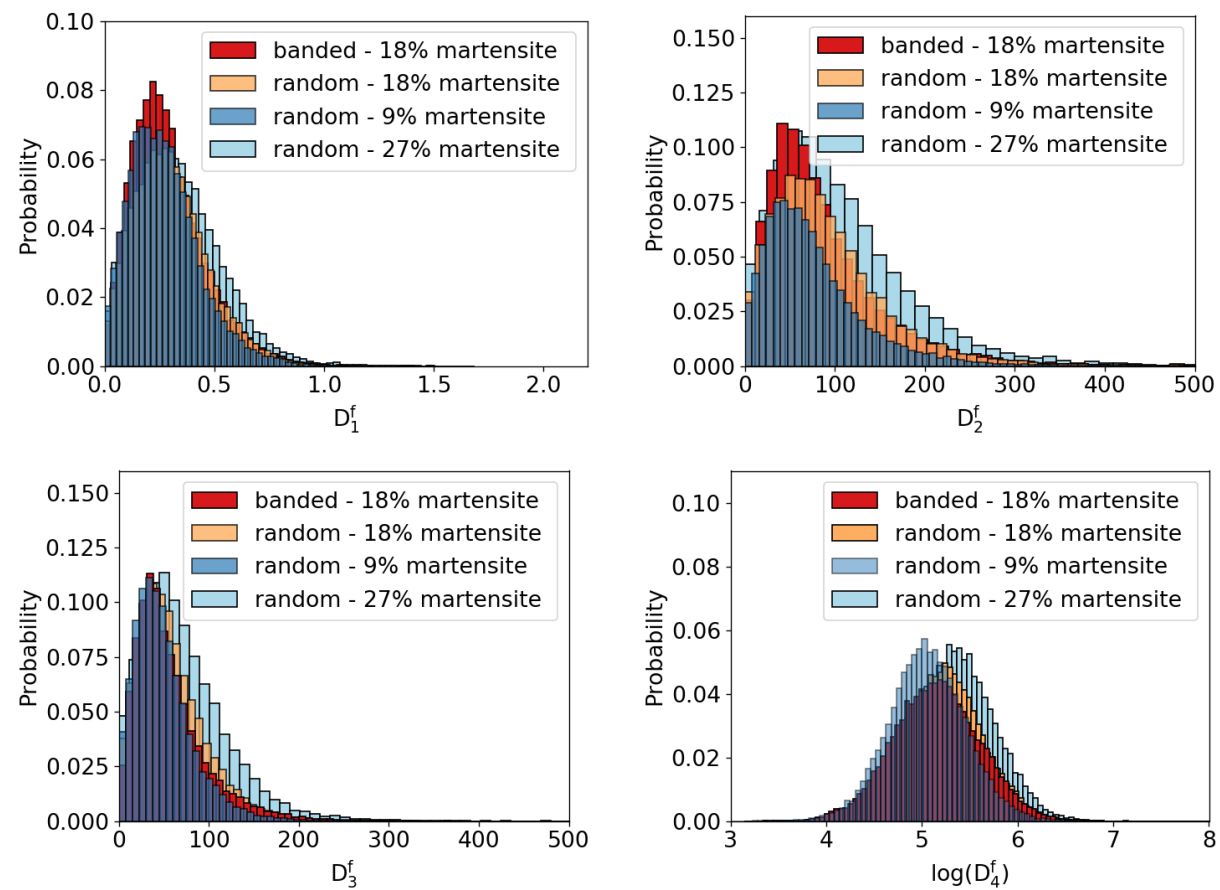

Figure 6.13: Histograms of $\mathrm{D}_{1}^{\mathrm{f}}, \mathrm{D}_{2}^{\mathrm{f}}, \mathrm{D}_{3}^{\mathrm{f}}$ and $\mathrm{D}_{4}^{\mathrm{f}}$. 
(a)

(b)

(c)

(d)
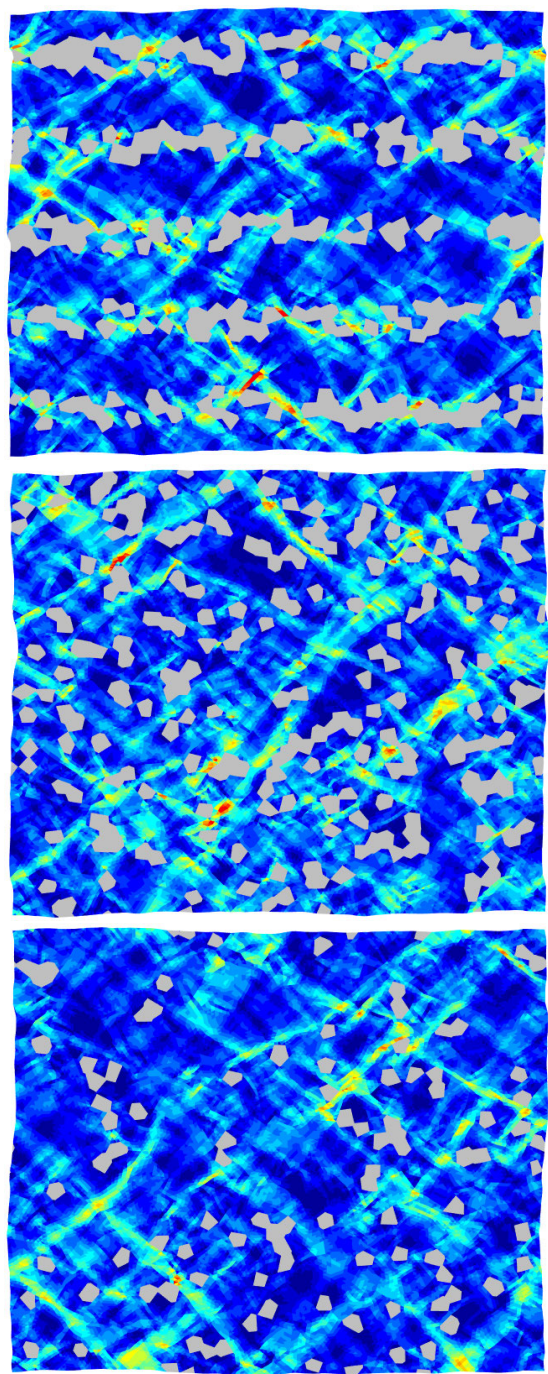

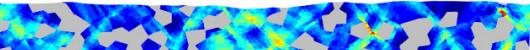

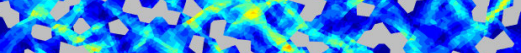

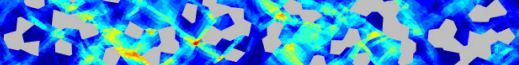

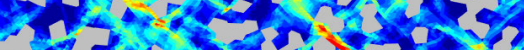

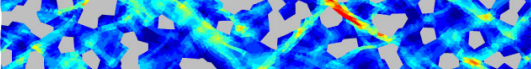
$x_{r} \rightarrow,+h, 2 a y+2$.

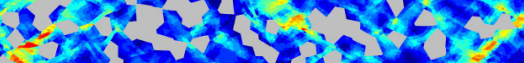

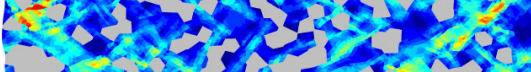

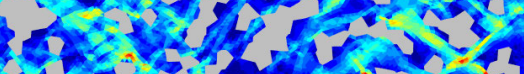

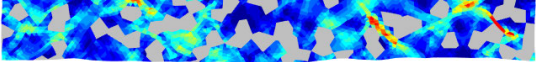
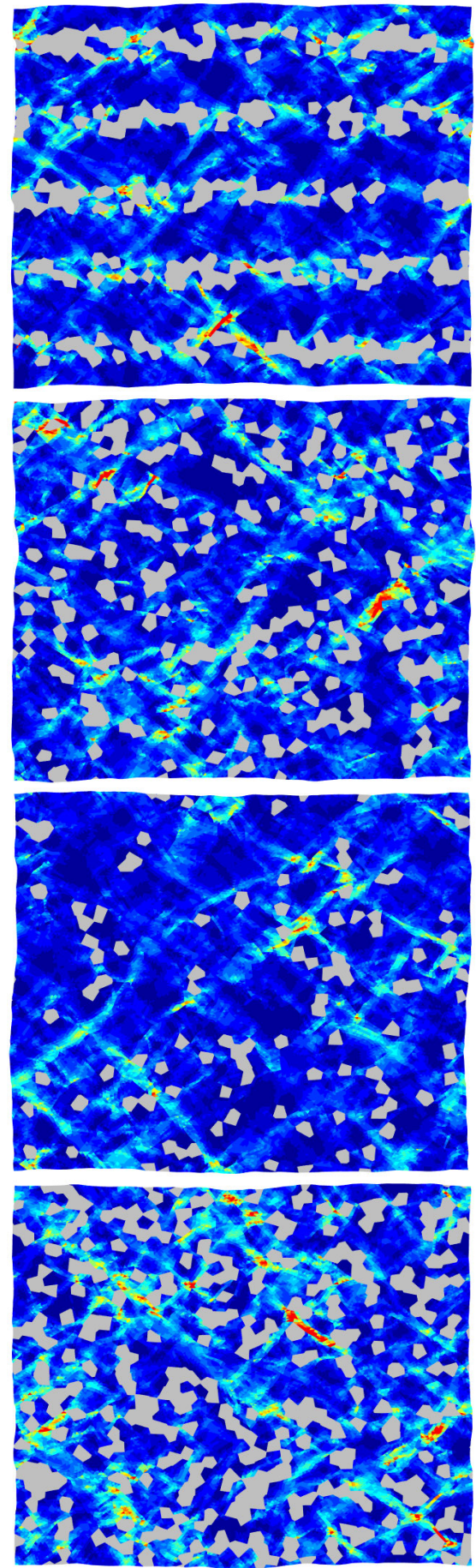

Figure 6.14: Distribution of damage indicators. Left: $\mathrm{D}_{1}^{\mathrm{f}}$, Right: $\mathrm{D}_{2}^{\mathrm{f}}$. (a) Banded 18\% martensite, (b) Random - 18\% martensite, (c) Random - 9\% martensite, (d) Random - 27\% martensite. 
(a)
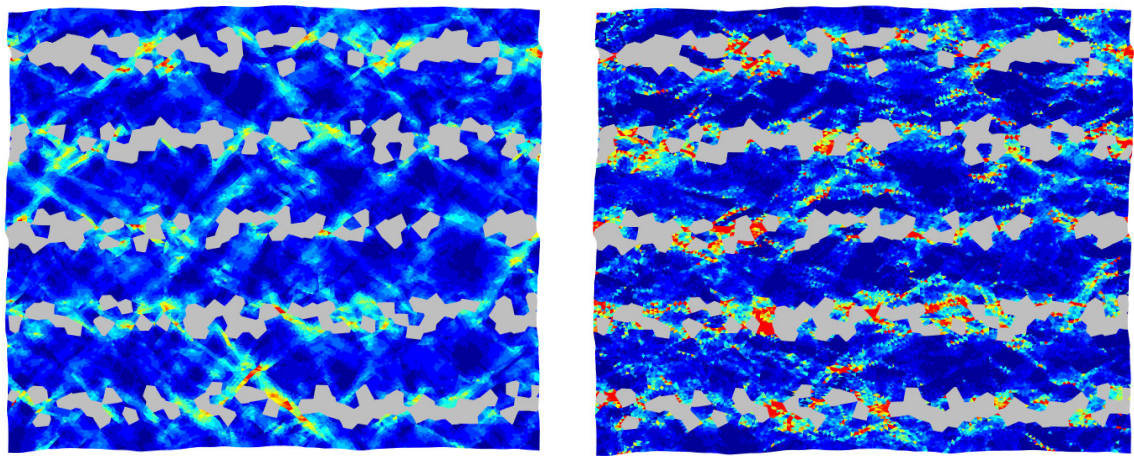

(b)
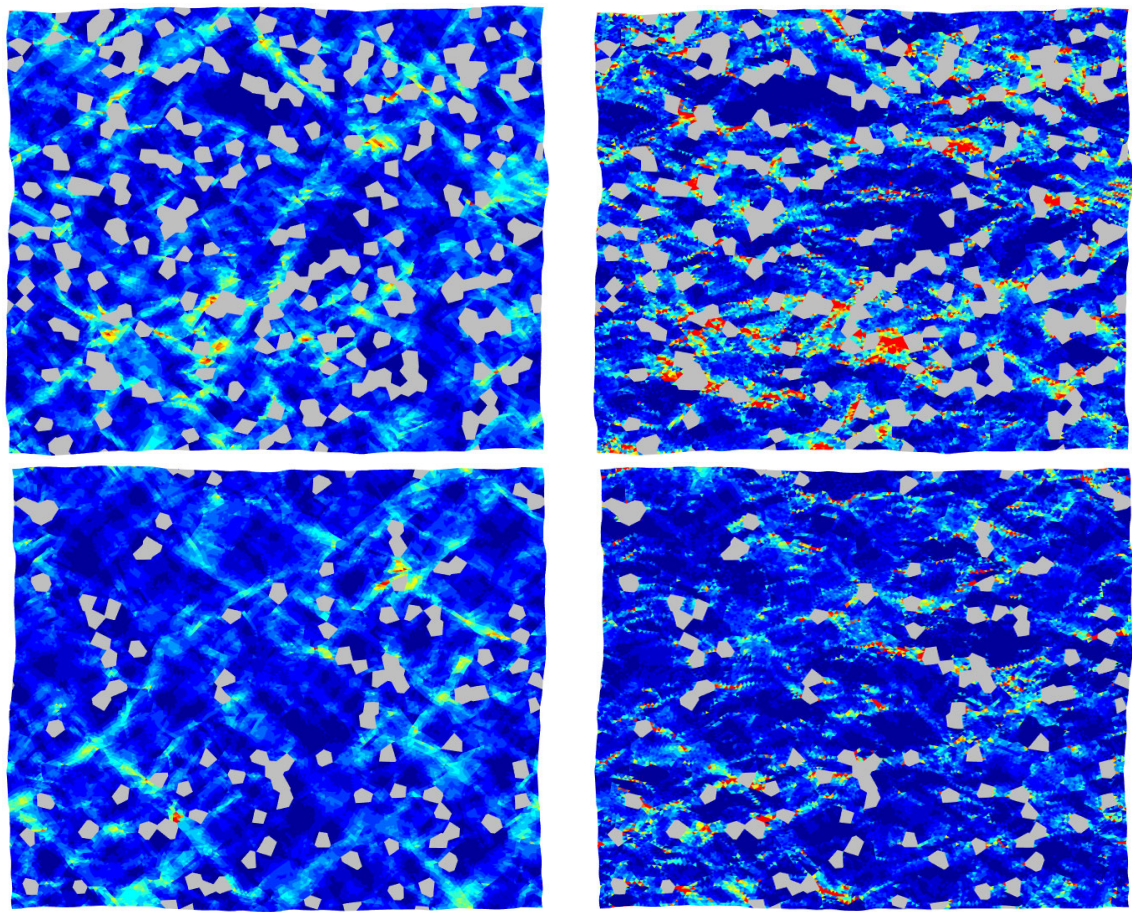

(c)

$(d)$
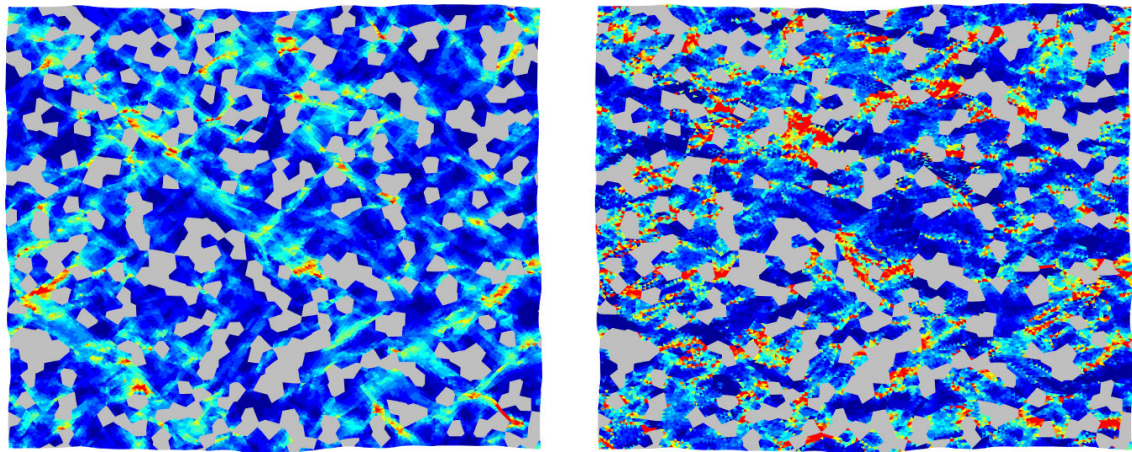

Figure 6.15: Distribution of damage indicators. Left: $\mathrm{D}_{3}^{\mathrm{f}}$, Right: $\mathrm{D}_{4}^{\mathrm{f}}$. (a) Banded 18\% martensite, (b) Random - 18\% martensite, (c) Random - 9\% martensite, (d) Random - 27\% martensite. 
of martensite, the indicators do not favor any of the morphologies. So the main factor effecting the damage in ferrite seems to be the amount of martensite, not the morphology (distribution) of martensite.

Figure 6.14 and 6.15 show distribution of the four damage indicators, $D_{1}^{\mathrm{f}}, \mathrm{D}_{2}^{\mathrm{f}}, \mathrm{D}_{3}^{\mathrm{f}}$ and $\mathrm{D}_{4}^{\mathrm{f}}$ for all the RVEs. The location of the hot spots of the indicators do not vary with martensite fraction or distribution but the number is affected. With increasing martensite content the increase is recognizable for all the indicators. The indicators, $\mathrm{D}_{2}^{\mathrm{f}}$ and $\mathrm{D}_{3}^{\mathrm{f}}$, are very similar in all conditions. $\mathrm{D}_{1}^{\mathrm{f}}$ is the most diffused and $\mathrm{D}_{4}^{\mathrm{f}}$ is the most localized indicator.

\subsection{Conclusions}

In this chapter, we have investigated numerically the effect of martensite distribution and fraction on the stress and strain partitioning behavior and damage initiation probability of dual phase steels by using the strain gradient enhanced crystal plasticity framework. It was shown that:

- The amount of martensite content with significant mechanical contrast affects the average stress and strain response of each phase due to the generation of GNDs

- Martensite distribution effects the partitioning of stress and strain into each phase. Stretch along the banding direction increases the tendency to iso-strainlike behavior.

- Both for ferrite and martensite phases a damage indicator based on plastic work gives reasonable hot spots to the experimentally observed damage sites. These occur for martensite at the phase boundaries and cutting across the islands and for ferrite at thin channels between martensite islands and next to martensite phase.

- Increase in martensite fraction and bandedness increases the probability of damage initiation in martensite.

- Increase in martensite fraction increases the probability of damage initiation in ferrite but the bandedness does not have a significant effect.

- The plastic work based damage indicators, $\mathrm{D}_{3}^{\mathrm{m}}$ and $\mathrm{D}_{3}^{\mathrm{f}}$, can be used during the development stage of dual phase steels in order to predict probable damage initiation locations in the microstructure. 


\section{Damage evolution in crystals}

In this chapter, void growth characteristics in single and dual phase microstructures are investigated within the strain gradient enhanced crystal plasticity framework. It was shown in Chapter 5 that stress and strain distributions exist in an RVE. The effect of this heterogeneous distribution on void growth is elaborated in Chapter 7 . Firstly, the effect of grain orientation, grain size and initial void size on void growth in a single phase material is investigated. Secondly, void growth in a dual phase material is studied. Three types of voids are considered: voids at the interface, voids in ferrite grains and voids formed by martensite cracking.

\section{$7.1 \quad$ Introduction}

The increasing trend toward lighter structures in the automotive industry requires multi-phase steels with complex microstructural morphologies, which triggers research not only into the production of these materials but also into the development of numerical tools to predict and evaluate material performance. Microstructural features, e.g. distribution and amounts of phases, grain size and shape variations, of these steels affect the stress and strain fields within the structure during deformation. Ductile failure behavior is highly dependent on the local conditions of stress and strain. It is, therefore, necessary to understand interrelated physical mechanisms that cause ductile material failure in a voided polycrystalline structure subjected to inelastic deformations.

Ductile fracture of crystalline metals is generally a result of nucleation, growth and coalescence of small internal voids. Void nucleation usually occurs by decohesion or fracture of second phase particles [167-169]. Once nucleated, voids evolve with plastic deformation in a stable manner until they start interacting with each other, leading

Some parts are based on:

EE Aşık, ES Perdahcıoğlu, and AH van den Boogaard.

An RVE based study of the effect of martensite banding on damage evolution in dual phase steels. Computational Materials Science, (submitted). 
later to coalescence and failure. The same story line holds for multi-phase alloys. For example, experimental studies on dual phase (DP) steels show that void nucleation occurs by cracking of martensite, between ferrite and martensite or within ferrite grains $[7,18,19,23,71,77,78]$.

Modeling of void evolution in ductile metals and the response of mechanical properties to void content, size and shape have been described in the literature starting with the early works on isolated void analyses of McClintock [170], Rice and Tracey [171] and the constitutive framework by Gurson [27]. Later, Koplik and Needleman [172] compared predictions based on Gurson's model with unit cell analyses. In these early studies, the matrix surrounding the void was assumed isotropic, although, recently plastic anisotropy of the matrix has been taken into account in unit cell calculations by employing either a Hill-type yield criterion or crystal plasticity formulations [50, 173-179].

Yerra et al. [176] did calculations on void growth in body-centered cubic (BCC) crystal under constant stress triaxiality. They observed strong dependence of void growth rate to on crystal orientation. Moreover, the study also showed that a higher stress triaxiality resulted in faster void growth. More recently, the study of Ling et al. [179] exhibited similar results for a face-centered cubic crystal. In their work, unit cell simulations were compared with the porous single crystal model of Han et al. [175] and they observed higher void growth rate for low triaxiality in the cell model. Shu et al. [178] investigated dependency of void size on void growth rate. They used a scale dependent elasto-viscoplastic framework for unit cell calculations and concluded that small voids had a tendency to grow slower than large voids. Moreover, Borg et al. [180] and Tvergaard et al. [181] concluded that void growth rate for small voids was suppressed for low stress triaxialities and the effect of orientation was more pronounced for small voids.

Void growth in metals has been studied at different length scales. At small scales, discrete dislocation dynamics (DDD) and molecular dynamics (MD) simulations are versatile tools to investigate the effect of lattice orientations and void size on determining the characteristics of porous single crystals. In the study of Segurado et al. [182], growth of voids in isolated face-centered cubic (FCC) single crystals under uniaxial and biaxial loading was investigated via DDD simulations. It was found that void growth was more dependent on lattice orientations in uniaxial stress state than in biaxial loading. In the study of Tang et al. [183], through MD simulations of void growth and coalescence in magnesium single crystals, it was shown that the pattern of plastic deformation, which was strongly dependent on crystal orientations and specimen size, had influences on void growth. Moreover, MD simulations in the study of Potirniche et al. [184], showed a higher increase of void fraction in smaller specimens, which was attributed to the development of larger local stresses.

Most of the previous works in literature for unit cell calculations use single crystals. However, in order to come up with a generalized understanding, representative features of an engineering material, e.g. phases, phase distributions, grain size, should be included in the model. The goal of this work is to clarify the effect of second phase distribution and grain orientations on the mechanics of void evolution in polycrystalline dual phase steel microstructures at mesoscopic scale. A strain 
gradient enhanced rate-independent crystal plasticity formulation is used in order to capture the size dependent, evolution of inhomogeneous plastic deformation. In this chapter, void evolution in 2D polycrystalline RVEs with initial void content is considered. Firstly, a single void in a single phase material is analyzed in terms of the effect of lattice orientation, grain size and initial void size. Secondly, RVEs with two constituent phases (ferrite and martensite) have been used to elucidate the behavior of voids in a DP steel under uniaxial loading condition. For the simulations, voids are introduced in the structure which are placed on the basis of the most common active damage mechanisms $[21,23]$. The cylindrical voids were placed to ferrite-martensite boundaries and into ferrite grains but being closer to martensite. Moreover, fully closed voids were introduced to the martensite islands in order to mimic the behavior of the voids formed by martensite cracking. In order to emphasize the effect of second phase distribution, two different microstructural morphologies are used. In one case a banded distribution of martensite is employed and for the other case randomly distributed martensite islands in a ferrite matrix are used. The first structure resembles the banded martensite distribution of commercial DP600 steels. On the other hand, the second distribution (random martensite distribution) is used to compare the evolution of different types of damage in order to clarify the effect of banding on damage evolution in DP structures. Furthermore, the effect of grain orientations is studied by using three different sets of random orientations for each type of microstructure. The results are investigated in terms of area change of individual damage events — voids at grains, martensite-ferrite interfaces and voids formed from cracked martensite islands - as well as the total evolution of the damaged area (area of the damage incidents).

\subsection{Void evolution in single phase polycrystals}

In this section, the evolution of a pre-existing void is considered with respect to lattice orientation, grain size and initial void size. The aim is to identify the microstructural aspects affecting void growth and to create background knowledge which will be used for the subsequent simulation of void growth in dual phase steels. As in Chapter 5,

Table 7.1: Material parameters for ferrite.

\begin{tabular}{cc}
\hline & Ferrite \\
\hline $\mathrm{E}(\mathrm{GPa})$ & 212 \\
$\nu(-)$ & 0.3 \\
$\tau_{0}(\mathrm{MPa})$ & 75 \\
$b(\mathrm{~mm})$ & $2.48 \times 10^{-7}$ \\
$\rho_{0}\left(\mathrm{~mm}^{-2}\right)$ & $7.50 \times 10^{6}$ \\
$\rho^{\infty}\left(\mathrm{mm}^{-2}\right)$ & $5.00 \times 10^{8}$ \\
$\gamma^{\infty}(-)$ & 0.3 \\
\hline
\end{tabular}




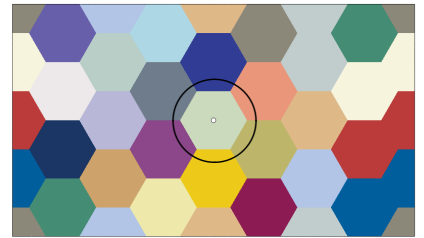

$\mathrm{O} 1, \mathrm{O} 4, \mathrm{O} 5, \mathrm{O} 6$

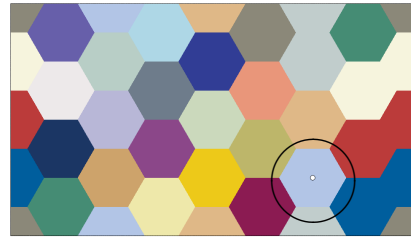

$\mathrm{O} 2$

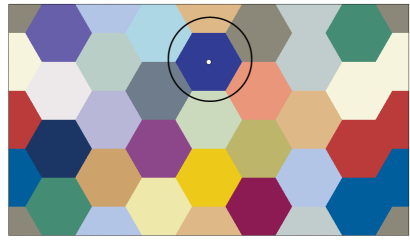

O3

Figure 7.1: RVEs consisting of grains with $12 \mu \mathrm{m}$ and the void located at different grains. Every crystal (represented by a different color) has a different crystallographic orientation. $\mathrm{O} 1$ is the original configuration. $\mathrm{O} 2$ and $\mathrm{O} 3$ are the variations where both the $1^{\text {st }}$ order neighbors and the orientation of the grain with the void are different. O4 to $\mathrm{O} 6$ are the configurations where the $1^{\text {st }}$ order neighbors have the same orientation but only the orientation of the grain with the void is different. Black circles show the grains that contain the void.

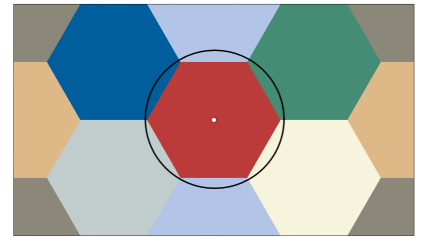

$d=24 \mu \mathrm{m}$

B1

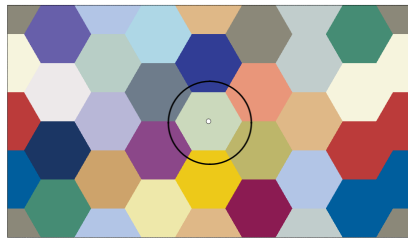

$d=12 \mu \mathrm{m}$

$\mathrm{B} 2-\mathrm{B} 4$

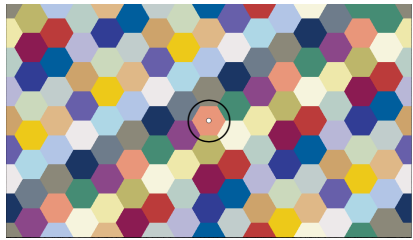

$d=6 \mu \mathrm{m}$

B3 - B5

Figure 7.2: Grain size of equally-sized RVEs used in the void growth simulations. Every crystal (represented by a different color) has a different crystallographic orientation. The same orientation is used for the grains with void in B1, B4 and B5. Black circles show the grains that contain the void.

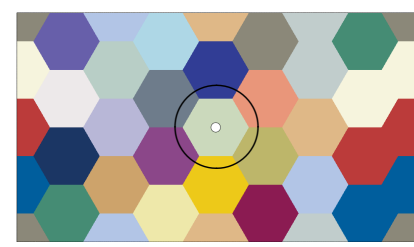

$$
d_{\mathrm{void}}=2 \mu \mathrm{m}
$$

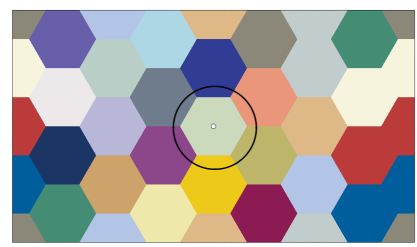

$d_{\text {void }}=1 \mu \mathrm{m}$

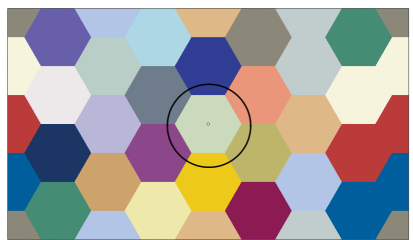

$d_{\text {void }}=0.5 \mu \mathrm{m}$

Figure 7.3: RVEs consisting of grains with $12 \mu \mathrm{m}$ and voids with different initial diameters. Every crystal (represented by a different color) has a different crystallographic orientation. Black circles show the grains that contain the void. 


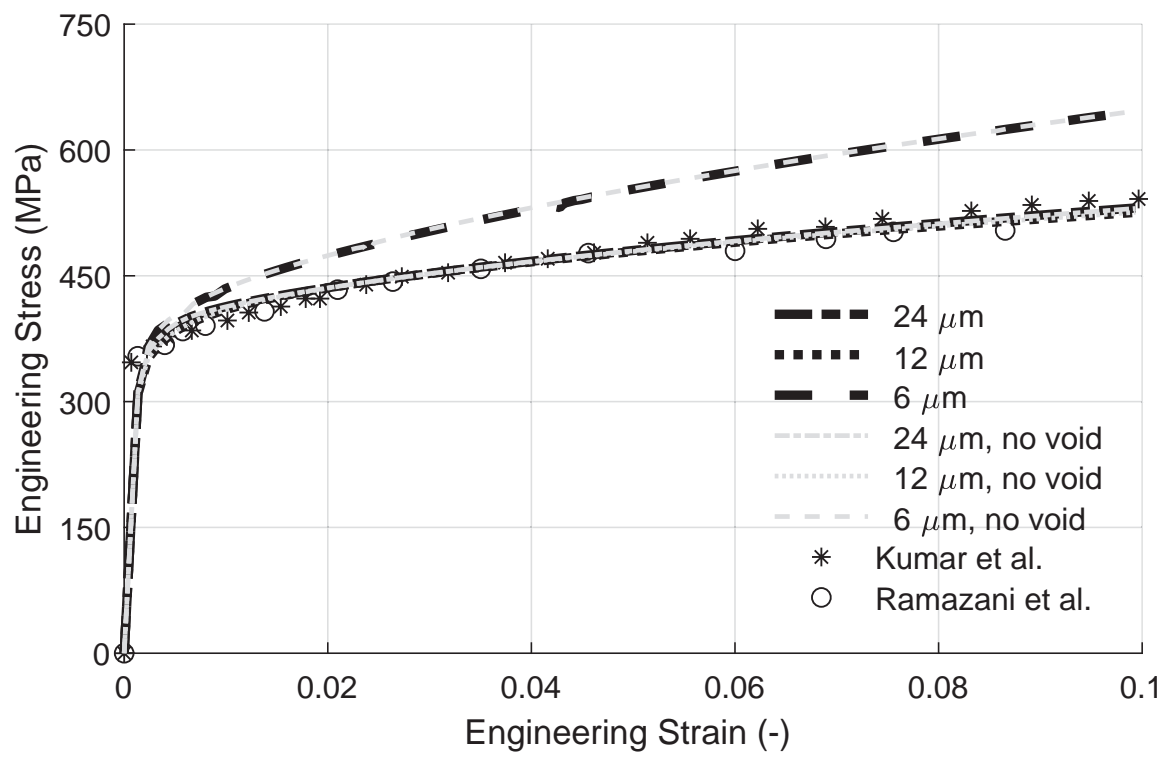

Figure 7.4: Engineering stress strain curves of the RVEs with different grain diameters with and without voids. Ferrite behavior from $[185,186]$.

equally-sized $(48 \times 83 \mu \mathrm{m}) 2 \mathrm{D}$ plane strain RVEs with hexagonal grains and random texture are used that are uniaxially stretched to $10 \%$.

Firstly, the effect of lattice orientation is considered for a single grain size $(12 \mu \mathrm{m})$ and a void with an initial diameter of $1 \mu \mathrm{m}$. However, it is not trivial to isolate the effect of lattice orientation of the grain with a pre-existing void on the evolution of that void during deformation due to grain-grain interactions. Changing the orientation of a grain will affect the behavior of its neighbors or, vice versa, if neighbors change, they will have an effect on the grain itself. Figure 7.1 shows the six different RVEs that are generated to investigate the effect of lattice orientation. The RVE identified as $\mathrm{O} 1$ is the control case which is also used to investigate other effects. In the RVEs identified as $\mathrm{O} 2$ and $\mathrm{O} 3$ the void is placed in different orientations. Because of the periodic structure and boundary conditions, the different position within the RVE does not influence the result. In these RVEs the orientation of both the neighboring grains and the grain with a void vary. In the RVEs identified as O4 to O6, orientations of the $1^{\text {st }}$ order neighbors is kept the same but the orientation of the grain with the void is different.

Secondly, we will consider the effect of grain size by employing three different grain sizes - 24, 12, $6 \mu \mathrm{m}$ - and a void with an initial diameter of $1 \mu \mathrm{m}$. Figure 7.2 shows the RVEs used and the initial void at the center. Finally, the evolution of voids with different initial diameters - 2, 1, 0.5 $\mu \mathrm{m}$ - are investigated. Figure 7.3 shows the RVEs with changing initial void content in the microstructure. In order to reduce the effect of lattice orientation on void evolution and give more emphasis on grain size, same orientation was used for the grains with the void in B1, B4 and B5. 

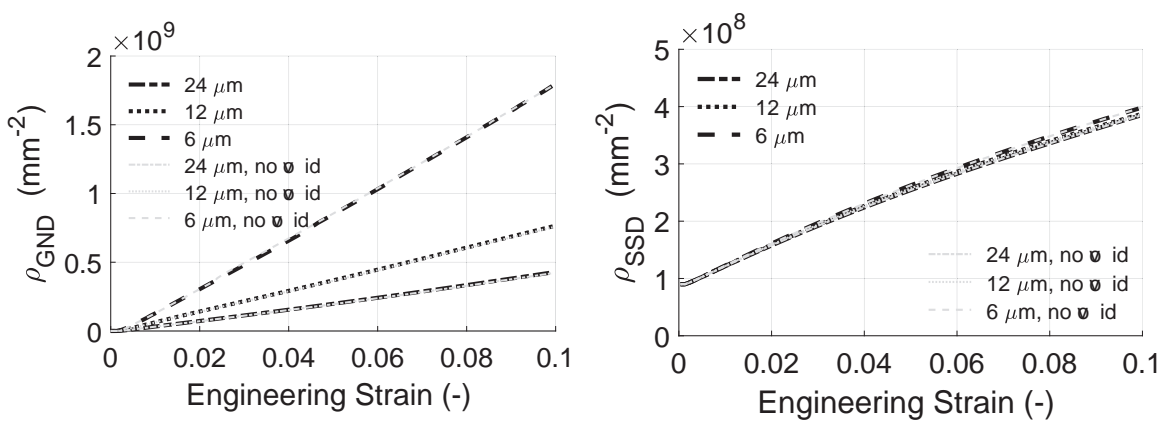

Figure 7.5: Evolution of GND (left) and SSD (right) densities of the RVEs with different grain diameters with and without voids.

A single set of material properties is used for all the simulations. The material properties were fit to represent the behavior of ferrite ( $\alpha$-iron) by using the stressstrain curves from Paul et al. and Ramazani et al. [185, 186]. Moreover, the initial dislocation density for ferrite is taken as $9.0 \times 10^{7} \mathrm{~mm}^{-2}$ for each slip system in ferrite as reported in the literature [164]. The fit was realized by using an RVE (without a void) with $12 \mu \mathrm{m}$ grain size. This fit also includes the GND effects due to the nature of the model. Table 7.1 shows the material properties. For the deformation of the BCC crystal only the $\langle 111\rangle\{110\}$ slip systems with tension-compression asymmetry (nonSchmid effects) is considered. The calculation method for the non-Schmid effects is given in Eq. (5.15). Moreover, the physically based interaction matrix $Q^{(\alpha \beta)}$ coefficients, given in Table 5.1, are used to define latent hardening due to SSDs and GNDs.

Figure 7.4 shows the stress-strain response of the RVEs with different grain sizes. The gray curves belong to RVEs without voids and the black curves are the responses of their voided counterparts. Moreover, the markers show the literature data that is used. From this figure, it can also be seen that grain size strengthening is not very dominant for the 24 and $12 \mu \mathrm{m}$ grain sized RVEs but has a clear effect for the $6 \mu \mathrm{m}$ grain size. In addition, since the void content is very small, the stress strain responses of the voided and non-voided RVEs are almost on top of each other. When we compare evolution of the average SSD density, we can also see that the trend is very similar for all RVEs. On the other hand, increasing trend in the average number of GNDs with a decreasing grain size can be seen from Figure 7.5.

\subsubsection{Effect of grain orientation on void evolution}

The effect of grain orientation is studied by comparing the RVEs with respect to stress-strain curve, the normalized void size and the spatial distribution of SSDs and GNDs. As can be seen from Figure 7.6, a different configuration of the RVE (O1 to O6) has negligible effect on the macroscopic stress-strain response of the RVE since there is statistically enough number of grains to tolerate the changes to the spatial 


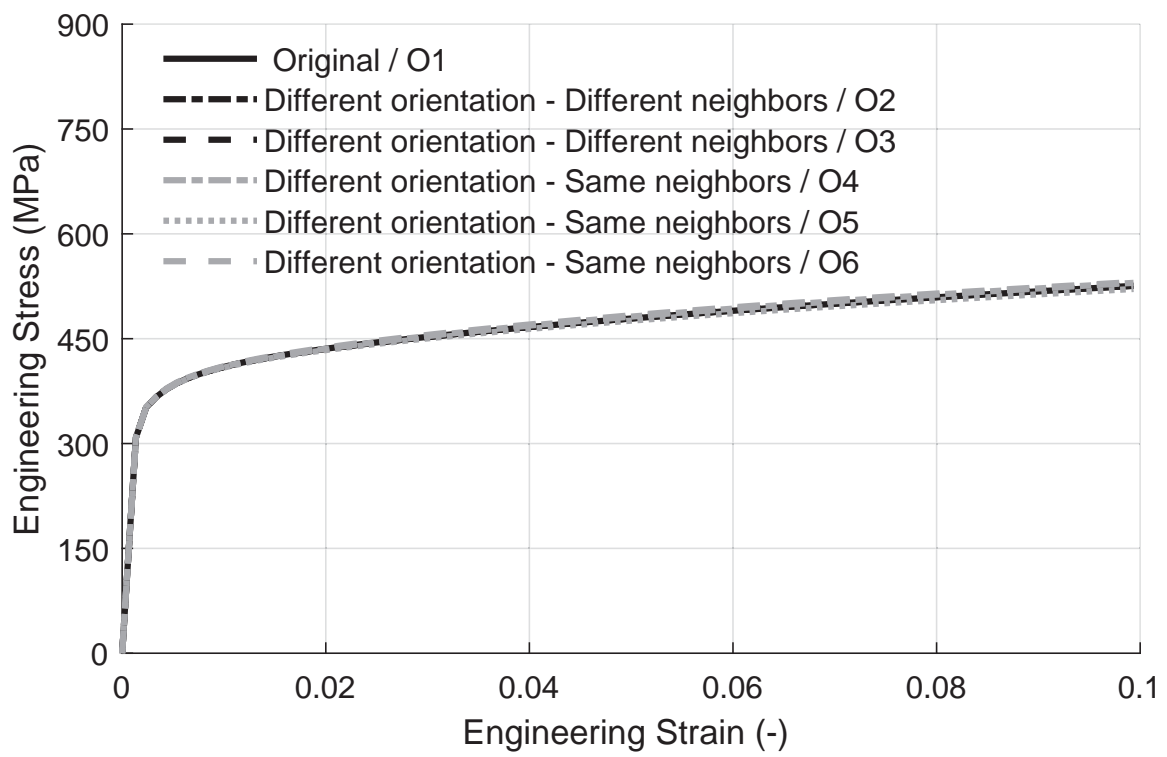

Figure 7.6: Engineering stress strain curves of RVEs with a grain size of $12 \mu \mathrm{m}$. The voids are located in different grains.

distribution of lattice orientation. It was also shown by Figure 7.4 that the initial void size was not big enough to cause a noticeable difference in the average response.

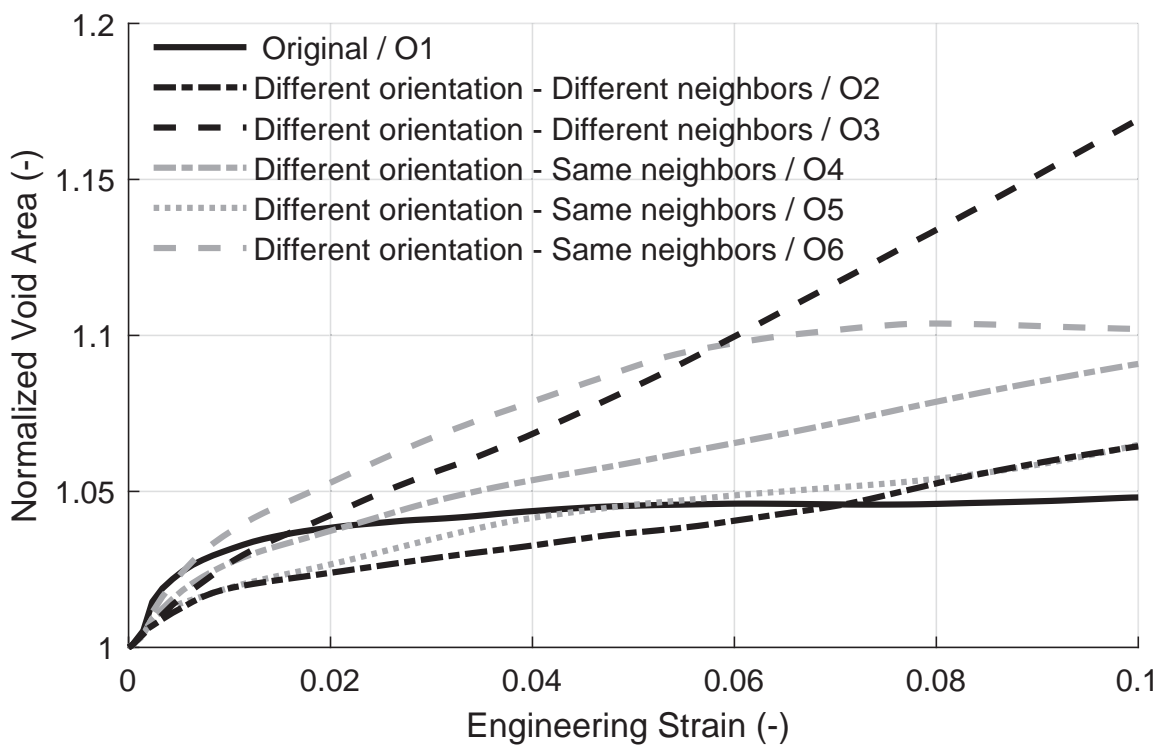

Figure 7.7: Evolution of normalized void area. 


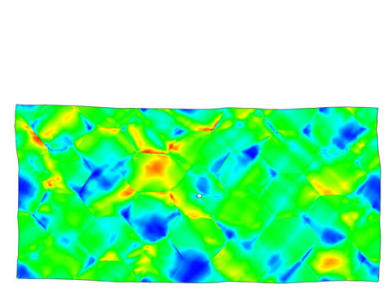

O1

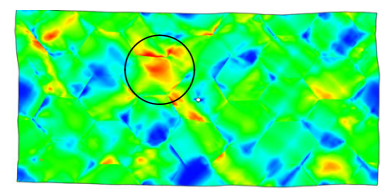

$\mathrm{O} 4$

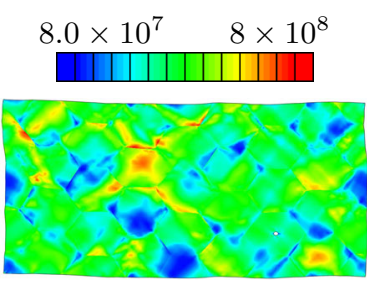

$\mathrm{O} 2$

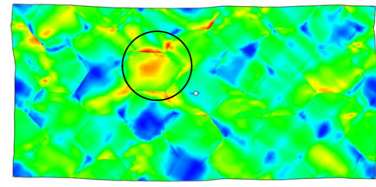

O5

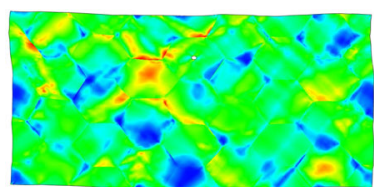

O3

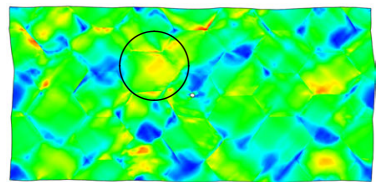

O6

Figure 7.8: Final distribution of SSDs $\left(\mathrm{mm}^{-2}\right)$ in the RVEs after tensile stretch of $10 \%$. In $\mathrm{O} 2$ and $\mathrm{O} 3$, both the $1^{\text {st }}$ order neighbors and the orientation of the grain with the void are different. In $\mathrm{O} 4$ to $\mathrm{O} 6$, the $1^{\text {st }}$ order neighbors have the same orientation as in O1; only the orientation of the grain with the void is different. The black circles show the effect of grain-grain interactions.

Figure 7.7 shows evolution of the normalized void area (ratio of the current area to the initial area) with increasing average strain of the RVEs. The figure shows curves that are exhibiting different characteristics. For example, curves that belong to O1 and O6 initially show a faster growth than the others but with increasing strain the growth rates decrease and even the growth of the voids almost stops. On the other hand, the voids in $\mathrm{O} 2, \mathrm{O} 3$ and $\mathrm{O} 4$ exhibit a behavior in a similar trend in the sense that they kept growing throughout the deformation but at different rates. When we compare $\mathrm{O} 1-3$ to $\mathrm{O} 1$ and $\mathrm{O} 4-6$, it is seen that the deviation in normalized size is larger for the configurations where lattice orientations of the $1^{\text {st }}$ neighbors change. These results show that void evolution is highly scattered and depends on the local conditions (stress, strain, orientation) of the grain and even grain-grain interactions play a significant role.

Figure 7.8 and 7.9 show the spatial distribution of SSDs and GNDs in the RVEs. Since the voids only affect a small region around them, the RVEs, which have the same spatial distribution of orientations (O1 - 3), show very similar GND and SSD distributions. On the other hand, the other RVEs (O4 - O6) contain some recognizable differences. An example of such a feature is shown with the black circles in Figure 7.8. This shows that locally the orientation of neighboring grains influences the plastic deformation and its distribution in a grain. 

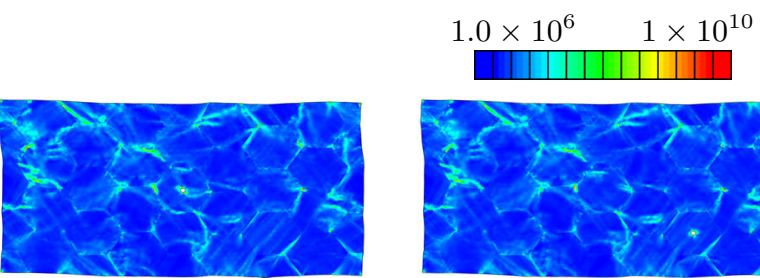

O1

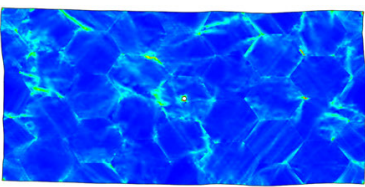

$\mathrm{O} 4$

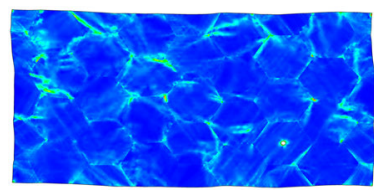

$\mathrm{O} 2$

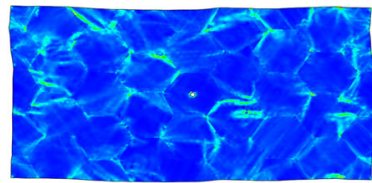

O5

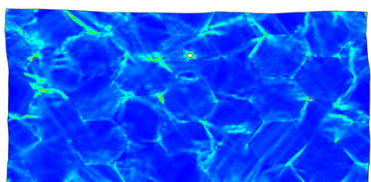

$\mathrm{O} 3$

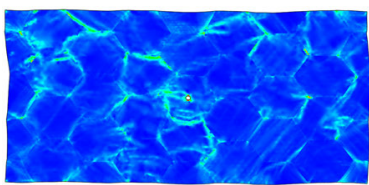

O6

Figure 7.9: Final distribution of GNDs $\left(\mathrm{mm}^{-2}\right)$ in the RVEs after tensile stretch of $10 \%$. In $\mathrm{O} 2$ and $\mathrm{O} 3$, both the $1^{\text {st }}$ order neighbors and the orientation of the grain with the void are different. In $\mathrm{O} 4$ to $\mathrm{O} 6$, the $1^{\text {st }}$ order neighbors have the same orientation as in $\mathrm{O} 1$; only the orientation of the grain with the void is different.

\subsubsection{Effect of grain size on void evolution}

Figure 7.10 shows the evolution of the normalized void area (ratio of the current area to the initial area) in the RVEs. The voids in the smallest grain size, B3 and B5, grow with an almost linear trend, whereas the voids in bigger grain sizes, B1 and B2, show a saturating type behavior. However, when the orientation of the grain with the void is changed, it is seen that the saturating trend in B2 turns into a rapidly growing trend in $\mathrm{B} 4$. This means that the effect is coming mostly from the orientations but not directly the grain size. A correlation in the void evolution is not found for the structures B1, B4 and B5 where the void is located at the grain with the same orientation.

Figure 7.11 shows the distribution of SSDs in the RVEs. With decreasing grain size, it is seen that a finer deformation pattern is formed in the structures, creating finer regions of high and low SSD density. The high SSD density also indicates high plastic deformation. As mentioned in Chapter 5, these regions inherently contain slip gradients that promotes GNDs. The SSD distribution around the voids do not show very specific characteristics but becomes more complex with decreasing grain size. This is mainly due to the refinement of the deformation pattern in the structure.

Figure 7.12 shows the distribution of GNDs in the RVEs. With decreasing grain size, more GNDs are observed at the grain boundaries and tripole junctions. Around the void there is a clearly defined high GND region. The size of this ring shaped region is biggest for the biggest grain size and smallest for the smallest grain size; however, this does not directly correlate to the evolution of void area. 


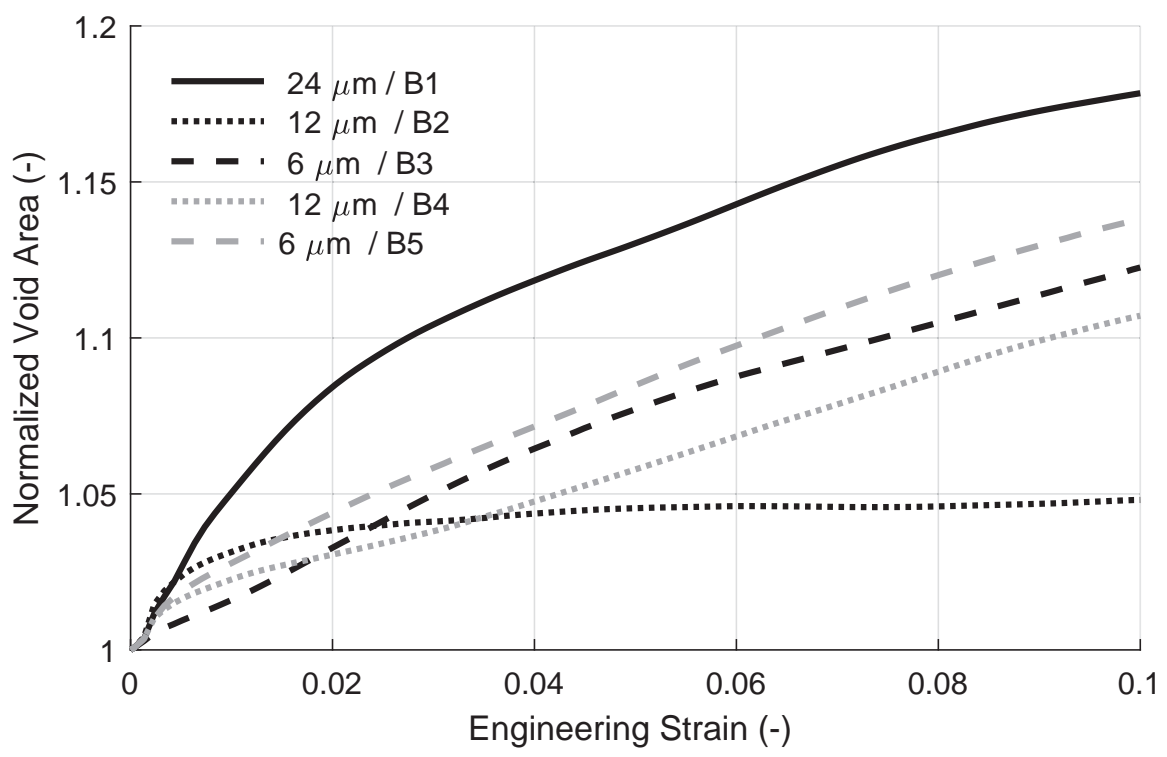

Figure 7.10: Evolution of normalized void area for different grain sizes. For B1, B4 and B5, the grain with the void has the same orientation.

\section{$8.0 \times 10^{7} \quad 8 \times 10^{8}$

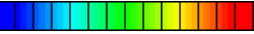

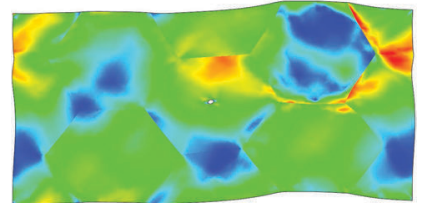

$$
d=24 \mu \mathrm{m} / \mathrm{B} 1
$$

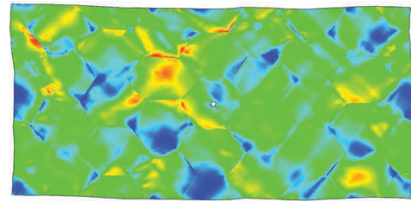

$d=12 \mu \mathrm{m} / \mathrm{B} 2$

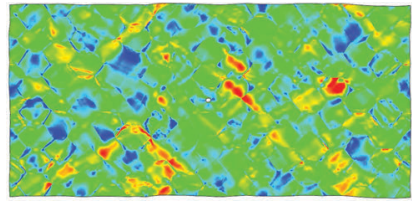

$d=6 \mu \mathrm{m} / \mathrm{B} 3$

Figure 7.11: Final distribution of SSDs $\left(\mathrm{mm}^{-2}\right)$ in the RVEs after tensile stretch of $10 \%$.

$1.0 \times 10^{6} \quad 1 \times 10^{10}$

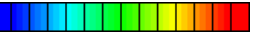

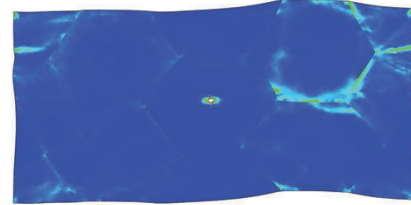

$$
d_{\text {grain }}=24 \mu \mathrm{m} / \mathrm{B} 1
$$

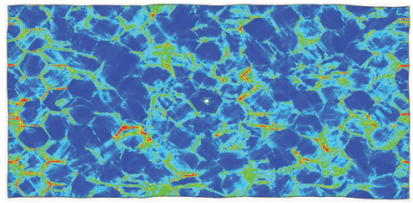

$$
d_{\text {grain }}=6 \mu \mathrm{m} / \mathrm{B} 3
$$

Figure 7.12: Final distribution of GNDs $\left(\mathrm{mm}^{-2}\right)$ in the RVEs after tensile stretch of $10 \%$. 


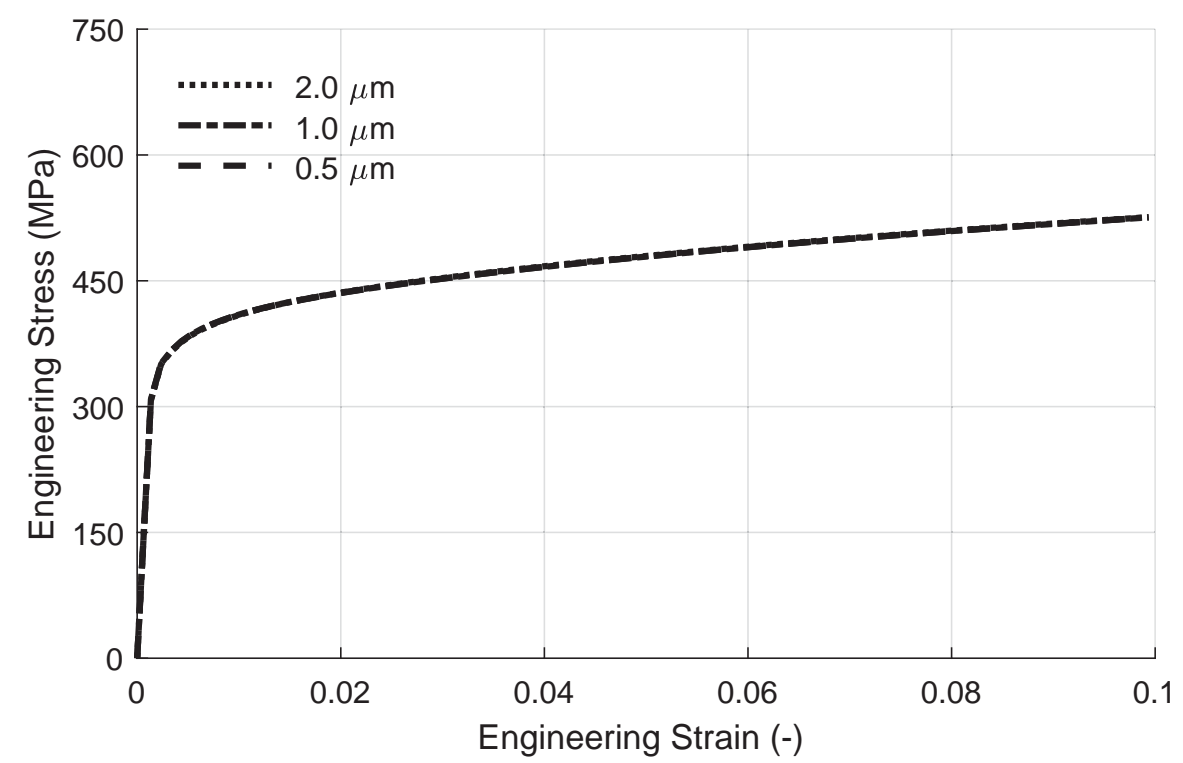

Figure 7.13: Engineering stress strain curves of the same RVE, with different initial void size.

\subsubsection{Effect of initial void size on void evolution}

The effect of initial void size is investigated by comparing RVEs with respect to the stress-strain curve, normalized void size evolution and spatial distribution of GNDs. These RVEs have the composed of grains with same orientations but with different intial void size. As can be seen from Figure 7.13, the initial void does not significantly alter the stress-strain curve due to the small size of the voids.

Figure 7.14 shows the evolution of different sized voids in the RVEs. In the three curves, there are two characteristic behaviors. For small voids, void area evolution shows a saturating type behavior. On the other hand, for the void with $2 \mu \mathrm{m}$ diameter, the evolution exhibits a continuously increasing trend. The faster growth of larger voids was also observed in the literature by Shu et al. [178]. They had found that the local strain gradients were increased when the voids were larger. This can also be seen from the GND distributions in Figure 7.15

Figure 7.15 shows the distribution of GNDs in the RVEs. The RVEs with 1 and 0.5 $\mu \mathrm{m}$ diameter voids show very similar GND distributions. The voids have only a local effect but the rest of the structure remains unaltered. With increasing void size, the high density GND region also grows. Moreover, with respect to the void with $2 \mu \mathrm{m}$ diameter effects, this zone is so large that it affects the neighboring grains. This can clearly be seen from the long band extending through the other grain. 


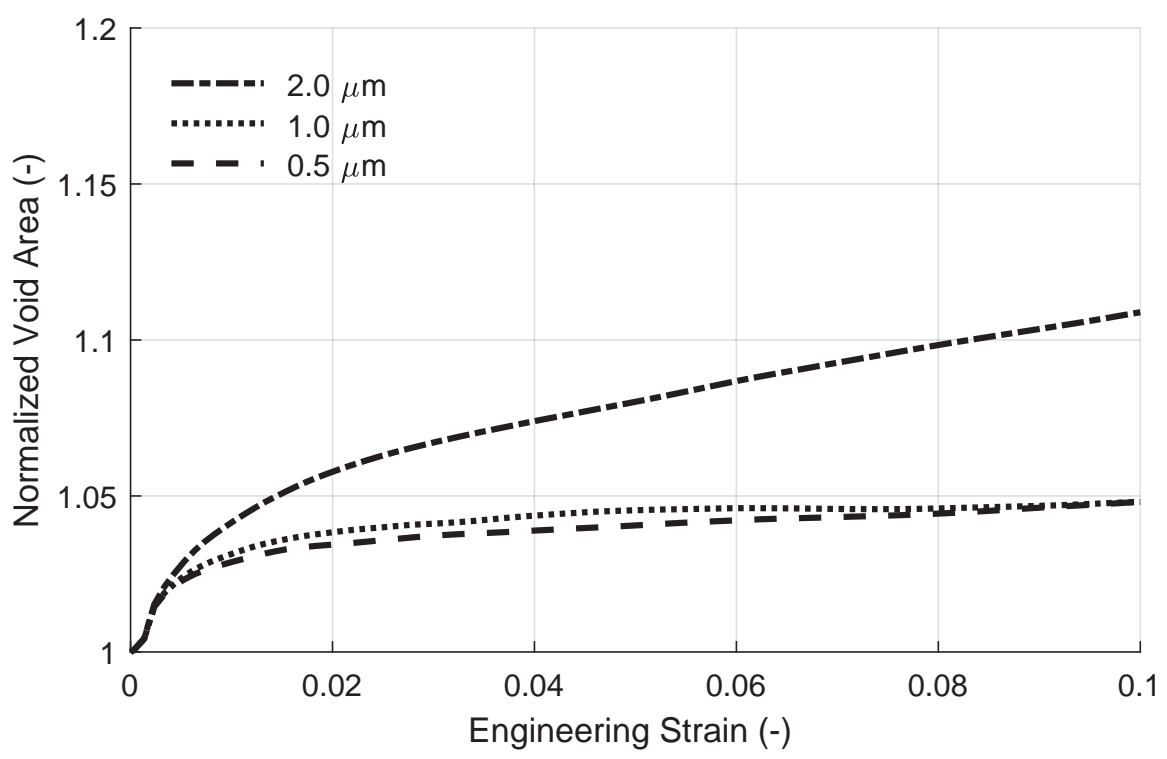

Figure 7.14: Evolution of normalized void area with different initial void size.

$1.0 \times 10^{6} \quad 1 \times 10^{10}$

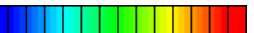
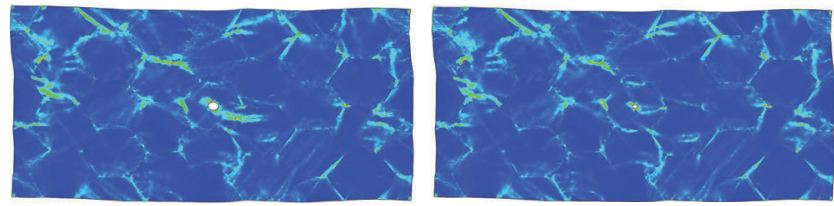

$d_{\text {void }}=2 \mu \mathrm{m}$

$d_{\text {void }}=1 \mu \mathrm{m}$

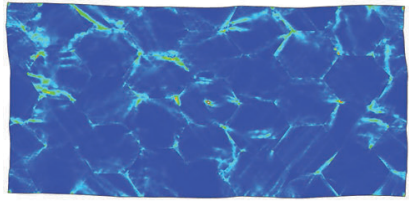

$d_{\text {void }}=0.5 \mu \mathrm{m}$

Figure 7.15: Final distribution of GNDs $\left(\mathrm{mm}^{-2}\right)$ in the RVEs after tensile stretch of $10 \%$. 


\subsection{Void evolution in dual phase steel}

In this section, 2D polycrystalline RVEs consisting of two phases (ferrite and martensite) have been used to investigate the evolution of voids in a DP steel under uniaxial loading conditions. Fully closed voids were introduced inside martensite islands, cutting through the complete island. Moreover, two types of cylindrical voids were placed at two different location types. The first type of location was the interphase boundaries between ferrite and martensite. The second type was inside the ferrite grains with a martensite neighbor and towards the boundary between ferrite and the neighboring martensite island. The choice of these locations was based on the experimental observations, which show that in DP steels there is more than one active damage mechanism $[21,23]$. By adding the most common damage mechanisms into the model we aim to clarify and give an explanation on how one of the mechanisms becomes dominant over the others and on the effect of martensite banding on the evolution of these damage mechanisms.

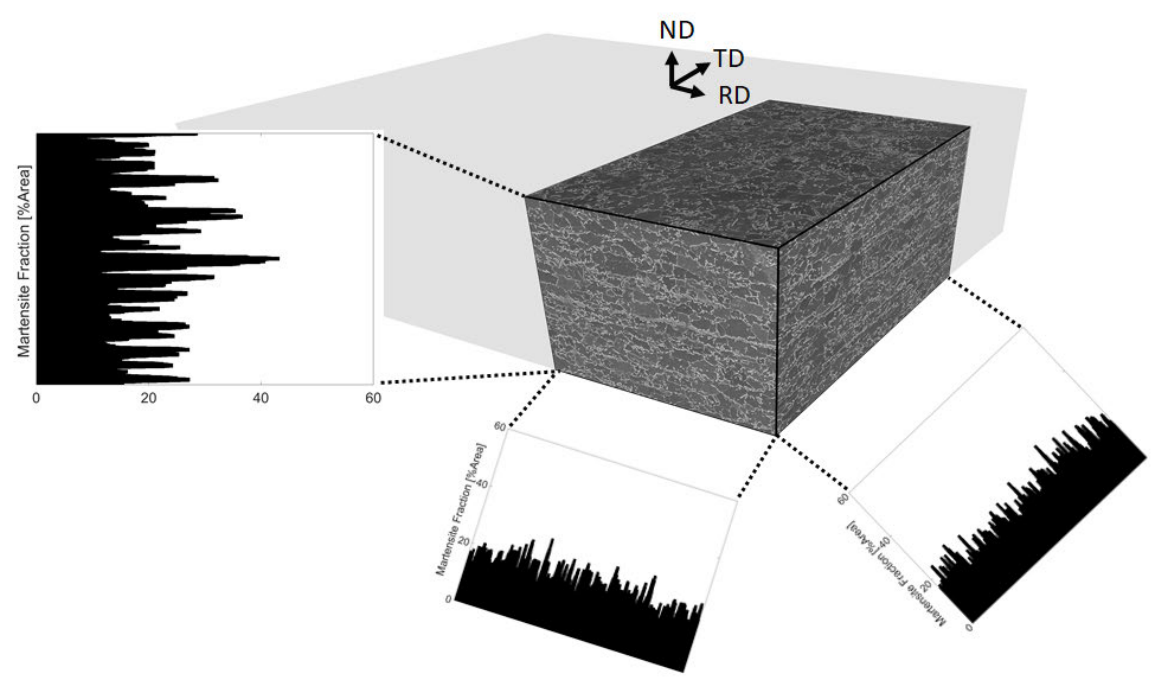

Figure 7.16: Typical microstructure of a commercial DP600 steel and distribution of vol\% martensite.

Two different martensite morphologies were considered, namely banded and randomly distributed structures. Throughout the section, we have used the word morphology to specifically mention distribution of martensite islands in the ferrite matrix. For readability we have not used the word distribution every time but it has always been implied unless mentioned otherwise. The RVE with banded morphology was generated by considering a commercial DP600 steel (Tata Steel, Ijmuiden, The Netherlands). Figure 7.16 illustrates secondary electron (SE) images obtained from different cross-sections of the steel and the measured volume percentages of martensite distribution along rolling, transverse and normal directions. The martensite content was calculated by image analysis and was found to be an average of $17.9 \pm 0.4 \mathrm{vol} \%$ 
martensite. From the martensite distribution charts, along the thickness direction a nonhomogeneous, banded distribution of martensite can be seen. The spacing between two martensite bands was measured to be roughly 10 to $15 \mu \mathrm{m}$ and in between the martensite bands there were two or three ferrite grains. Moreover, grain analysis by electron backscattered diffraction revealed an average ferrite grain area of $22.5 \mu \mathrm{m}$ and a martensite grain area of $5.6 \mu \mathrm{m}^{2}$. According to these measurements an RVE with a size of $40 \times 40 \mu \mathrm{m}$ and containing $18.14 \mathrm{vol} \%$ martensite was generated, as shown in Figure 7.17, to represent a banded DP600 steel. For representing the RVE with random martensite morphology, grain sizes were generated with the same parameters and this yielded an RVE with a martensite content of 17.85 vol\%, see Figure 7.18. The martensite morphologies of the generated RVEs can be seen in Figure 7.17(b) and Figure 7.18(b), where the color black represents the martensite phase. Moreover, Figure 7.17(c and d) and Figure 7.18(c and d) show the ferrite and martensite grains. Orientations of the grains in both RVEs were assumed to be random and orientation variations within a martensite island was not taken into account. For each RVE, three randomly generated orientations sets were used in order to compare damage evolution in the structure statistically.

The RVEs were generated by using the Microstructure DEsign TOOL: Multilevel Voronoi (MLV) tessellation software developed by Tata Steel. In comparison with the standard Voronoi tessellation, the Multilevel Voronoi technique enables generating complex grain structures and phase distributions, which results in convex shaped grains. The basic principle of the technique is based on regrouping fine Voronoi structures by using a coarser tessellation depending on location of the seed points of fine Voronoi cells. The reader may refer to the works of Kok et al. [187].and Yadegari et al. [188] for more detailed explanations on the multilevel Voronoi approach as well as the possibilities it presents in microstructure generation. The MLV software generates output files, that contain geometrical and orientation information to create microstructures. These files were used to create grains with defined orientations as parts in ABAQUS/CAE by using the scripting language PYTHON.

After RVEs were generated, different types of voids were introduced to the structure in the graphical user interface of ABAQUS/CAE and meshed with quadratic triangular plane strain elements (CPE6M). Both RVEs contain nine voids at the ferrite martensite interface and nine voids inside ferrite grains with a radius of $0.3 \mu \mathrm{m}$ and six completely cracked martensite grains, as shown in Figure 7.17(a) and Figure 7.18(a), making an initial void percentage $\left(\% f_{0}\right)$ of 0.32 . The initial void size was selected in such a way as to represent voids which are nucleated but have not grown much. The RVEs were subjected to $15 \%$ uniaxial elongation by applying periodic boundary conditions.

\subsubsection{Parameter identification}

Since the aim of the simulations was to investigate the effect of martensite distribution on damage evolution in DP600 steels, the material properties of the phases were fitted to the macroscopic stress-strain response of ferrite and martensite that was used by Ramazani et al. [185] in modeling dual phase steels. Parameter identification was 

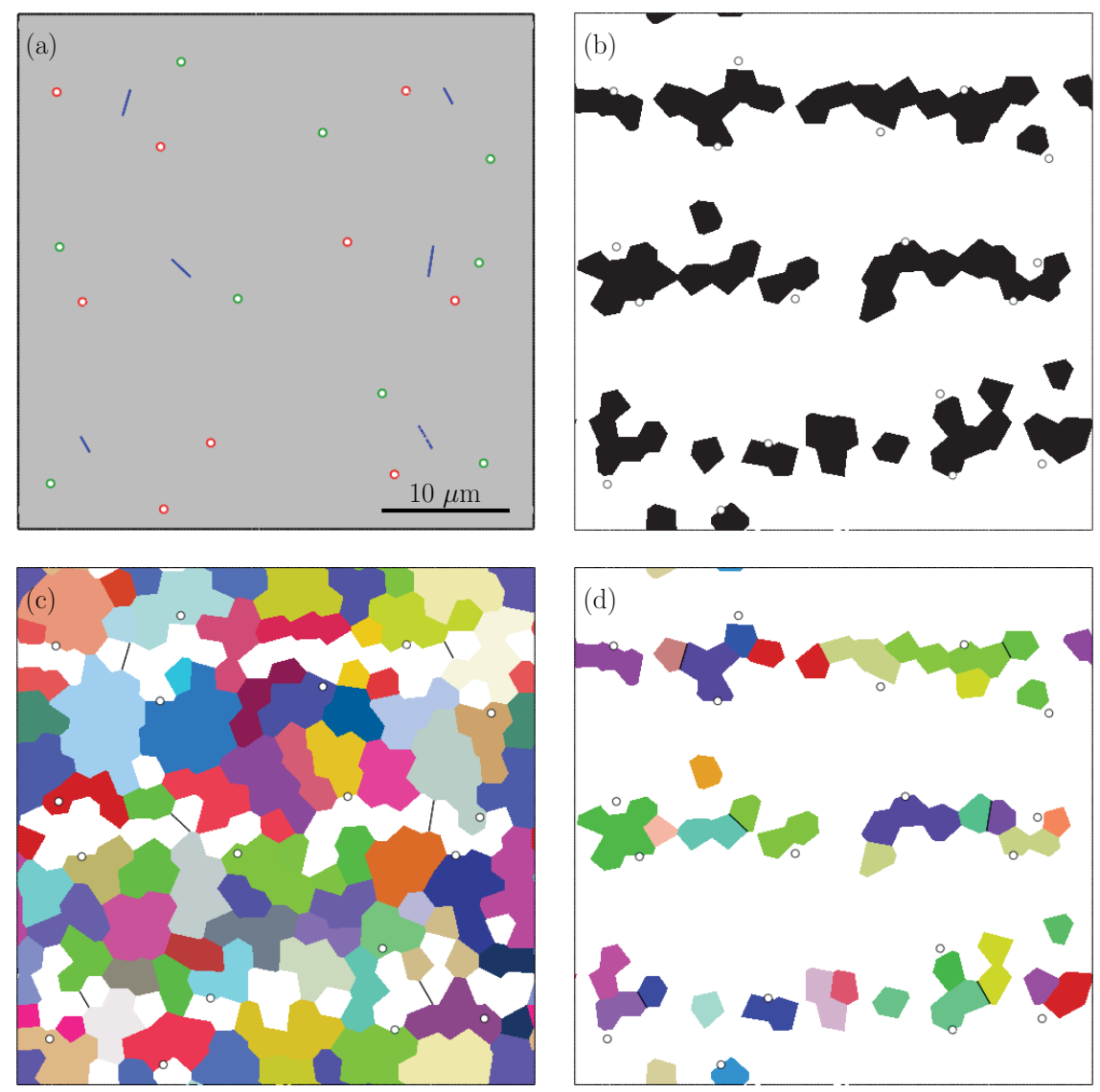

Figure 7.17: RVE with banded morphology, (a) voids (green: voids in ferrite, red: voids at the ferrite-martensite boundary) and cracks (blue), (b) ferrite (white) and martensite (black), (c) colors indicate 69 ferrite orientations, (d) colors indicate 29 martensite orientations.

done by employing an RVE consisting of either ferrite or martensite as represented in Figure 7.19 .

For each phase, a Voronoi-based microstructure with 160 grains was generated and the RVEs were subjected to $7.5 \%$ uniaxial tensile stretch, while periodic boundary conditions were imposed. As the constitutive model used in this study is size dependent the grain size of the single phase RVEs were generated to have similar grain sizes of constituent phases as the RVEs with dual phase structures and the simulations were repeated with three different sets of random orientations to check the representativity. Moreover, in the current fitting the initial dislocation densities for each slip system of each phase were calculated from the total dislocation density that is reported in literature as $9.0 \times 10^{7} \mathrm{~mm}^{-2}$ for ferrite [164] and $1.6 \times 10^{9} \mathrm{~mm}^{-2}$ for martensite [165]. These densities were assumed to be equally distributed to 

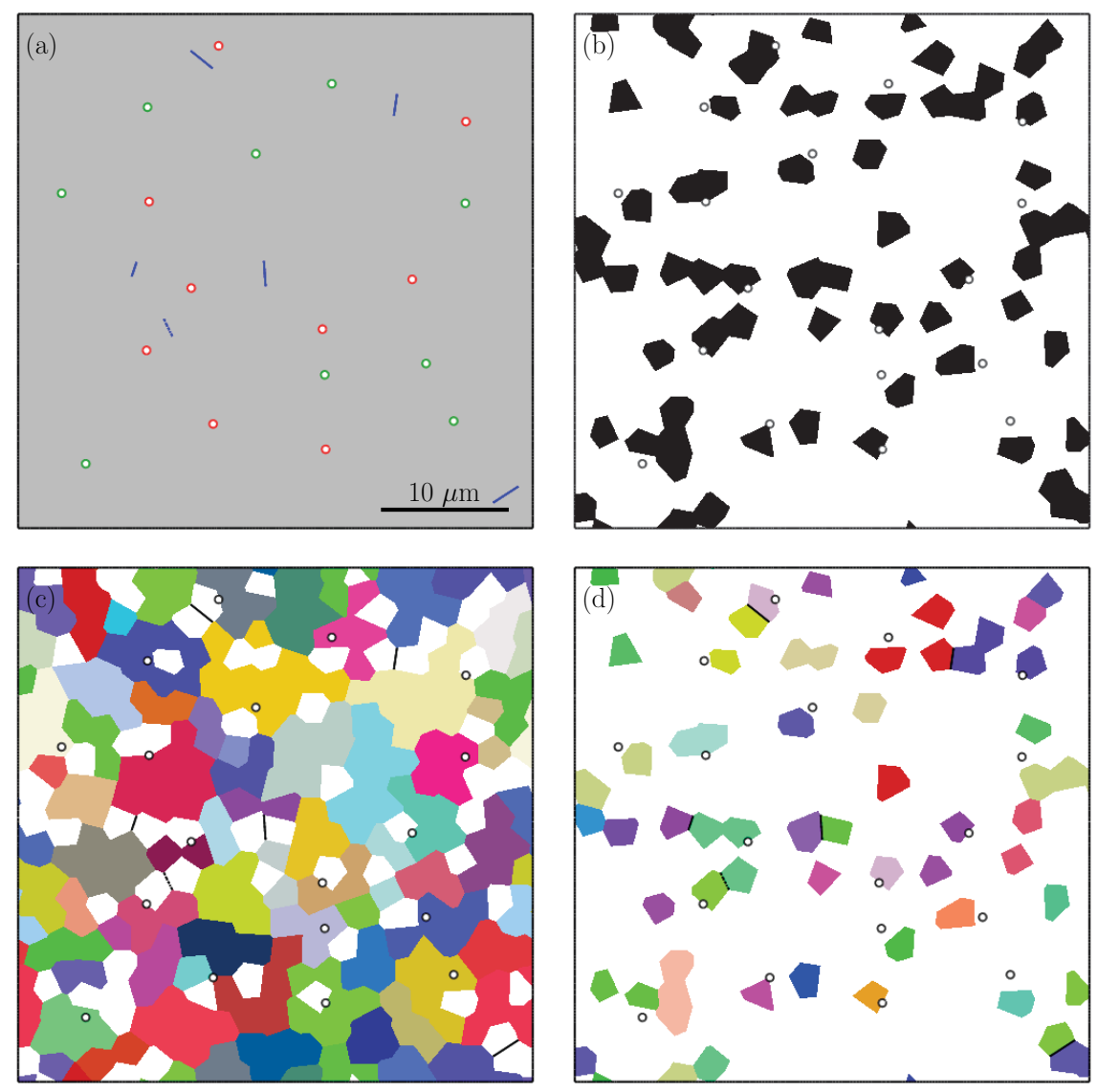

Figure 7.18: RVE with random morphology, (a) voids (green: voids in ferrite, red: voids at the ferrite-martensite boundary) and cracks (blue), (b) ferrite (white) and martensite (black), (c) colors indicate 67 ferrite orientations, (d) colors indicate 32 martensite orientations.

the 12 possible slip systems yielding dislocation densities of $7.50 \times 10^{6} \mathrm{~mm}^{-2}$ and $1.34 \times 10^{8} \mathrm{~mm}^{-2}$ for each slip system of ferrite and martensite, respectively. The other parameters $\left(\tau_{0}, \rho^{\infty}\right.$ and $\left.\gamma^{\infty}\right)$ given in Table 7.2 were used as fitting parameters. Mechanical response of RVEs with only ferrite or martensite is shown in Figure 7.20 with markers. Additionally, the figure also shows stress strain curves of RVEs with banded and random morphologies and an experimentally measured uniaxial DP600 curve. With the current RVE size, we see that the stress response of DP600 structures shows a maximum scatter of $3 \%$, which is sufficiently low for this study. However, if necessary, this scatter can be reduced by using larger sized RVEs at the cost of a longer solution time. 

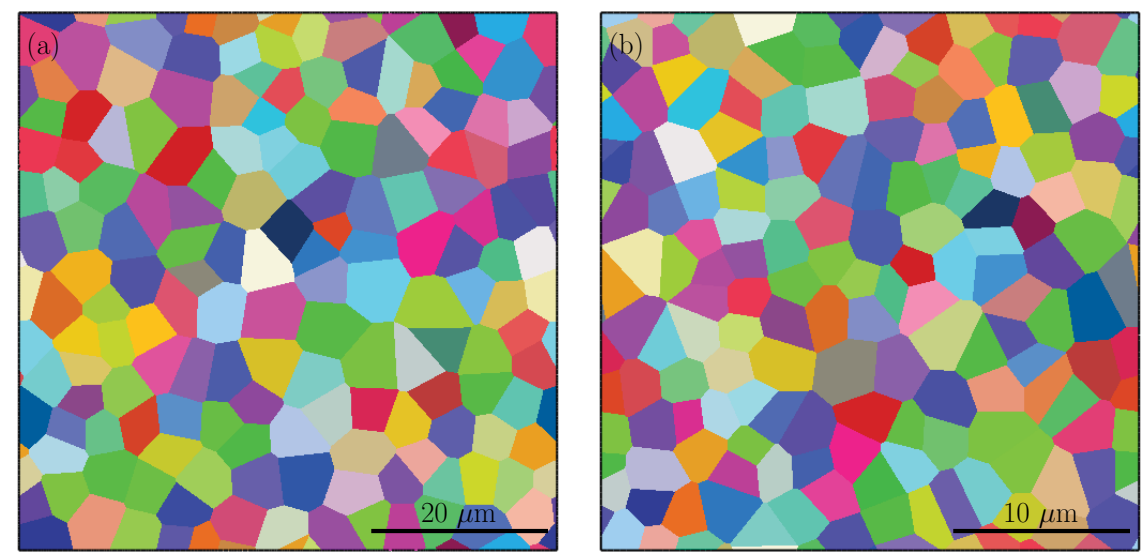

Figure 7.19: RVEs used to model (a) ferrite, (b) martensite. Colors represent the Voronoi cells with different grain orientations.

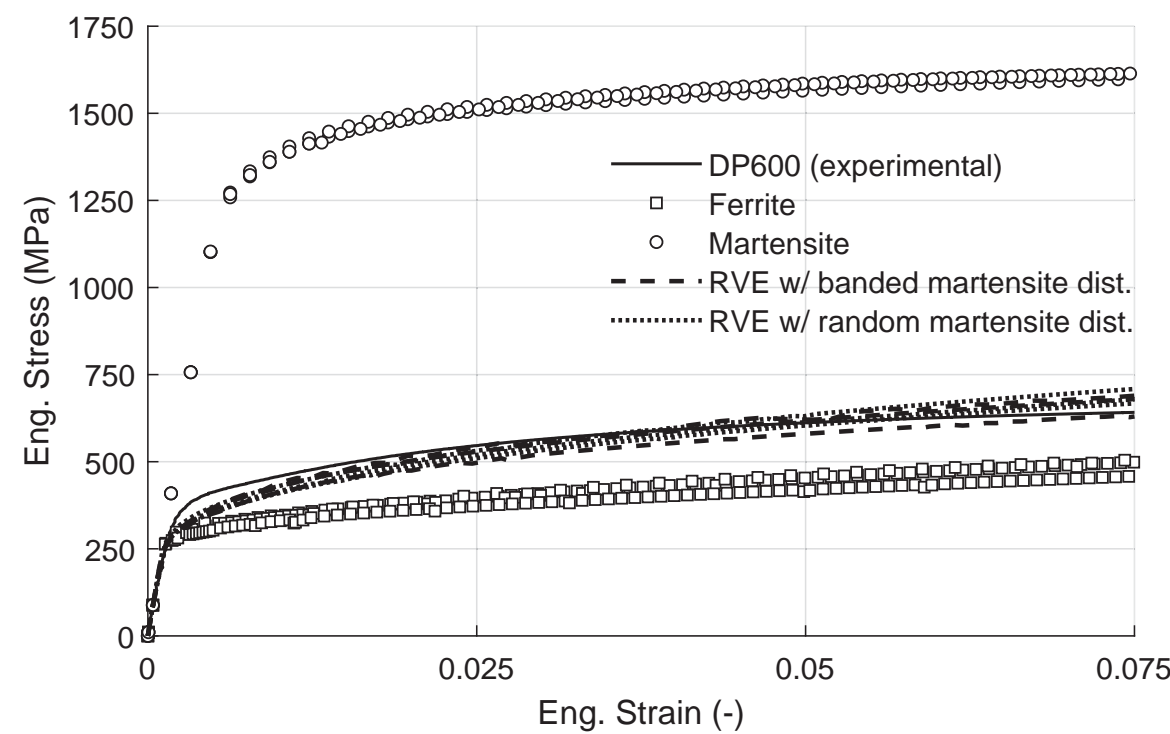

Figure 7.20: Stress-strain response of DP600 (experimental), individual phases and the different morphologies.

\subsubsection{Evolution of dislocation densities}

Here we compare the distribution of dislocation densities after deformation. Two different scales were used for Figure 7.21 (a,d and g) and Figure 7.25 (a,d and g) due to the large difference between the initial statistically-stored dislocation (SSD) density of ferrite and martensite. For both morphologies, deformation is not uniform but it concentrates in certain regions and forms shear bands. The shear bands can 
Table 7.2: Material parameters for ferrite and martensite

\begin{tabular}{ccc}
\hline & Ferrite & Martensite \\
\hline $\mathrm{E}(\mathrm{GPa})$ & 212 & 212 \\
$\nu(-)$ & 0.3 & 0.3 \\
$\tau_{0}(\mathrm{MPa})$ & 40 & 250 \\
$b(\mathrm{~mm})$ & $2.48 \times 10^{-7}$ & $2.48 \times 10^{-7}$ \\
$\rho_{0}\left(\mathrm{~mm}^{-2}\right)$ & $7.50 \times 10^{6}$ & $1.34 \times 10^{8}$ \\
$\rho^{\infty}\left(\mathrm{mm}^{-2}\right)$ & $3.00 \times 10^{8}$ & $5.00 \times 10^{8}$ \\
$\gamma^{\infty}(-)$ & 0.4 & 0.3 \\
\hline
\end{tabular}

be identified from the distribution of SSD density, which accommodates and evolves with plastic strain. The main difference in the SSD density of two morphologies is the distribution of these bands. In the random morphology, shear bands form a finer pattern than in the banded one. Ferrite in between the martensite islands has to deform to accommodate the prescribed deformation since the mechanical strength contrast between the two phases is large, so the ferrite phase in any orientation is softer than martensite. The presence of a higher number of ferrite channels between the martensite islands in the random morphology yields finer shear band structure. However, the long and continuous martensite bands restrict the plastic flow causing coarser shear bands.

The effect of martensite distribution and shear band formation can also be evaluated from the evolution of average SSD and GND densities in different phases. Figure 7.22 show that the average SSD evolution is almost similar for all the cases where there is formation of higher GND density for the RVEs with random morphology. Figure 7.23 shows the change in SSD density in ferrite and martensite. The higher average SSD density of ferrite in the random morphology suggests accommodation of a greater amount of plastic strain than the ferrite phase in the banded morphology. In the meantime, the martensite phase strains less and yields a smaller amount of SSD density. From a stress point of view, this means that the continuous martensite bands carry more load than the martensite islands in the random morphology. The scatter due to different orientation sets can also be investigated. It is seen that the average normalized SSD density in ferrite of banded morphology has a scatter of $6.0 \%$ at 0.15 longitudinal strain, whereas that of random morphology is $1.7 \%$, which means orientations in a banded structure play a more important role than a random morphology. On the other hand, scatter in average SSD density in martensite for both morphologies is much smaller at a value of $0.5 \%$.

The GND density distributions can be compared by using Figure 7.21 (b,e and h) and Figure 7.25 (b,e and h). All the figures show clear localization of GND density around voids and at the tips of cracks. In addition, moderate GND densities $\left(5 \times 10^{8} \mathrm{~mm}^{-2}\right)$ in ferrite are present at the grain boundaries. From Figure 7.24, it is seen that the average GND density of ferrite evolves faster in the random morphology. This can 
be related to the finer structure of shear bands and higher $\operatorname{SSD}\left(\rho_{\mathrm{SSD}}^{\alpha}\right)$ content of random morphology. First, through the width of a shear band, there exists a gradient of slip resulting in GND accumulation. Since there are more shear bands in random morphology, they cause larger number of GNDs. Secondly, ferrite deforms more in random morphology as indicated by the higher SSD $\left(\rho_{\mathrm{SSD}}^{\alpha}\right)$ content. This increases the heterogeneity of plastic deformation and the GND content in ferrite. This trend is also observed in the average GND content of martensite. The greater amount of plastic deformation in the martensite phase in the banded structure than in the martensite islands of random morphology causes development of higher strain gradients. This is due to inhomogeneous distribution of plastic deformation within the martensite.

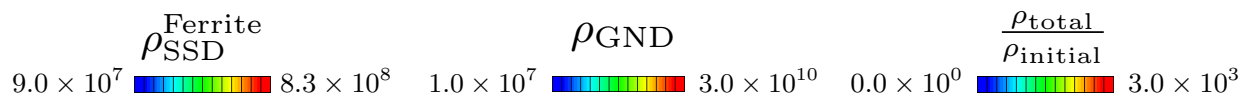

$\frac{\rho_{\text {SSD }}^{\text {Martensite }}}{1.6 \times 10^{9}} 2.8 \times 10^{9}$
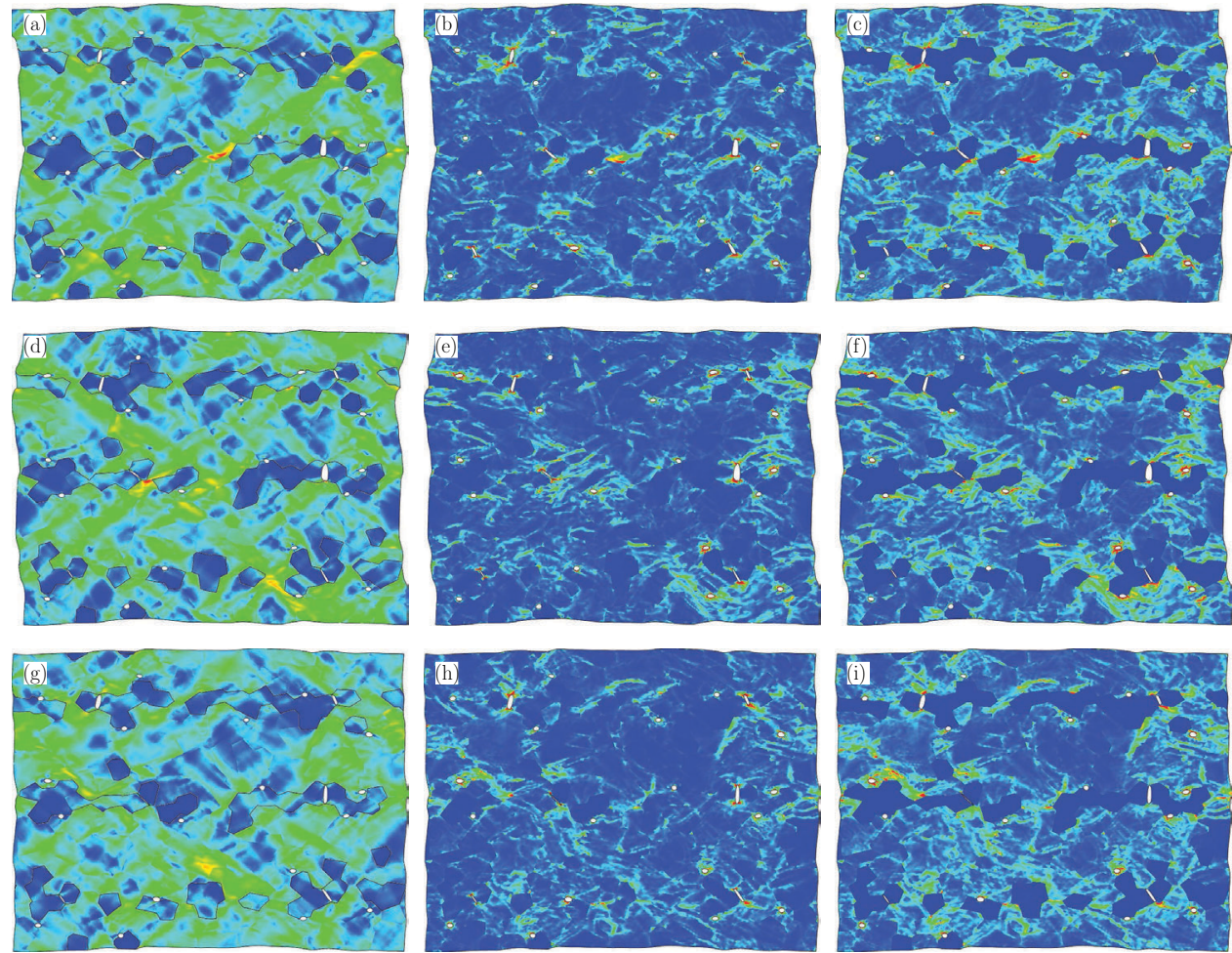

Figure 7.21: Distribution of (a,d and g) $\rho_{\mathrm{SSD}}\left(\mathrm{mm}^{-2}\right)$, (b,e and h) $\rho_{\mathrm{GND}}\left(\mathrm{mm}^{-2}\right),(\mathrm{c}, \mathrm{f}$ and i) Normalized dislocation density for banded morphology. Three rows represent three different orientation sets. 

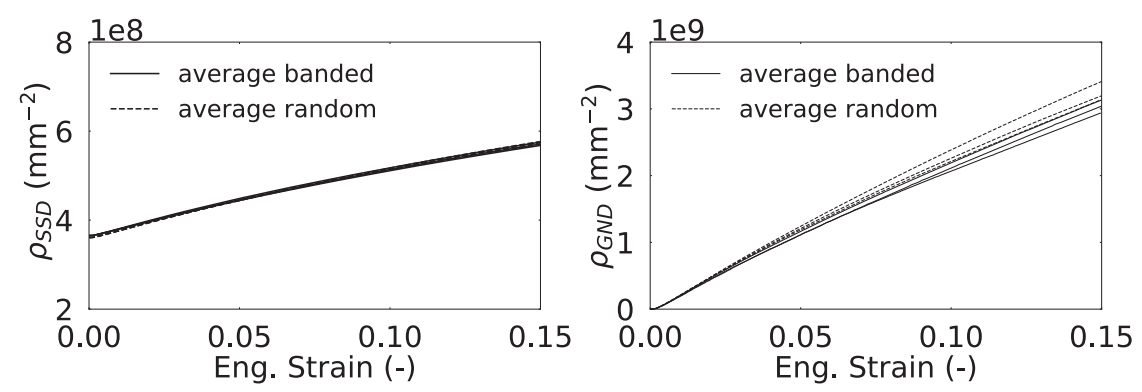

Figure 7.22: Average SSD (left)and GND (right) densities in the RVEs.
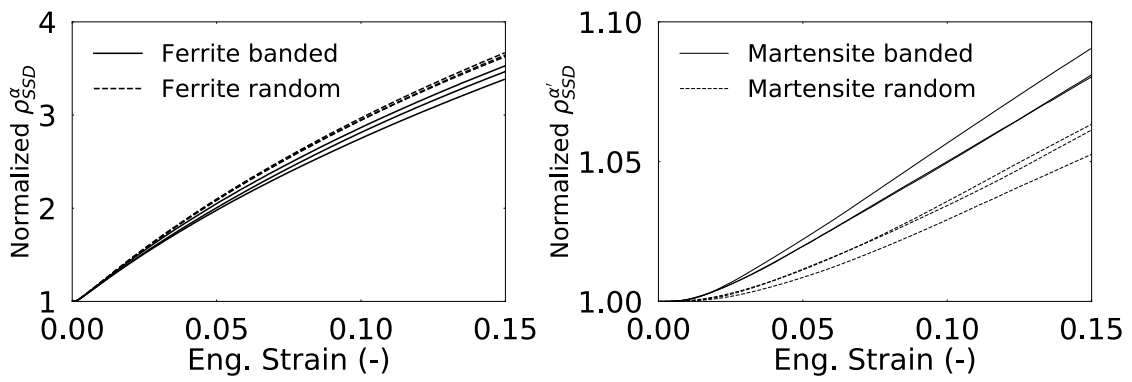

Figure 7.23: Evolution of normalized SSD density in the RVEs for ferrite (left) and martensite (right).

Figure 7.21 (c,f and i) and Figure 7.25 (c,f,i) show the distribution of normalized total dislocation densities with respect to the initial dislocation density $\left(\rho_{\mathrm{SSD}}\right)$ of the structure. In this way, the intensity of dislocation density due to the strength contrast between phases and the high density caused by presence of voids and cracks were made clear. It is seen between the figures that, the dislocation density varies with different orientation sets, even if the average stress strain curves are within a range of $3 \%$. For example, the top right corner of Figure 7.21 (c) has less dislocations than Figure 7.21 (f). However, there are also similarities. In all figures there are large regions of ferrite which are deformed less than the average of the structure. Moreover, around the damage occurrences, the dislocation density is always higher than in the rest of structure due to excess plastic deformation caused by stress concentration. 

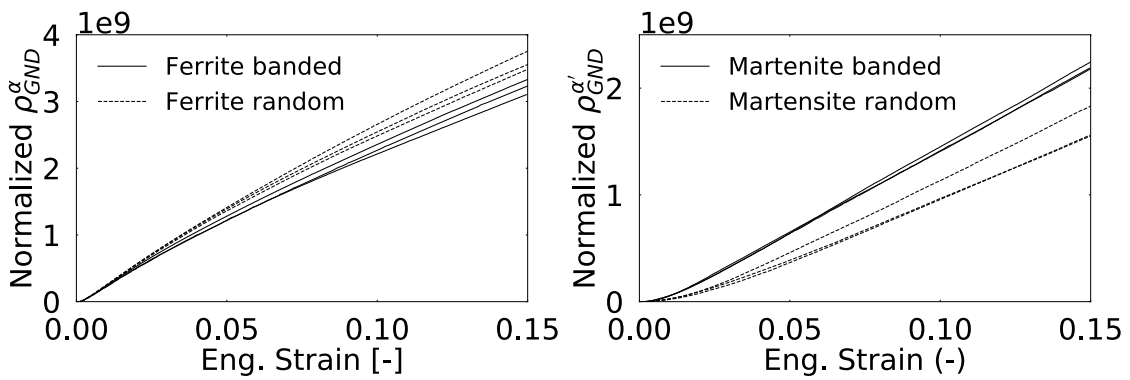

Figure 7.24: Evolution of normalized GND density in the RVEs for ferrite (left) and martensite (right).

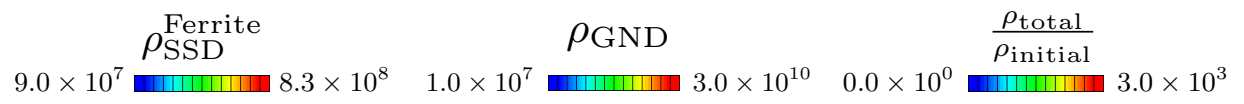

$\rho_{\mathrm{SSD}}^{\text {Martensite }} 1.6 \times 10^{9}$
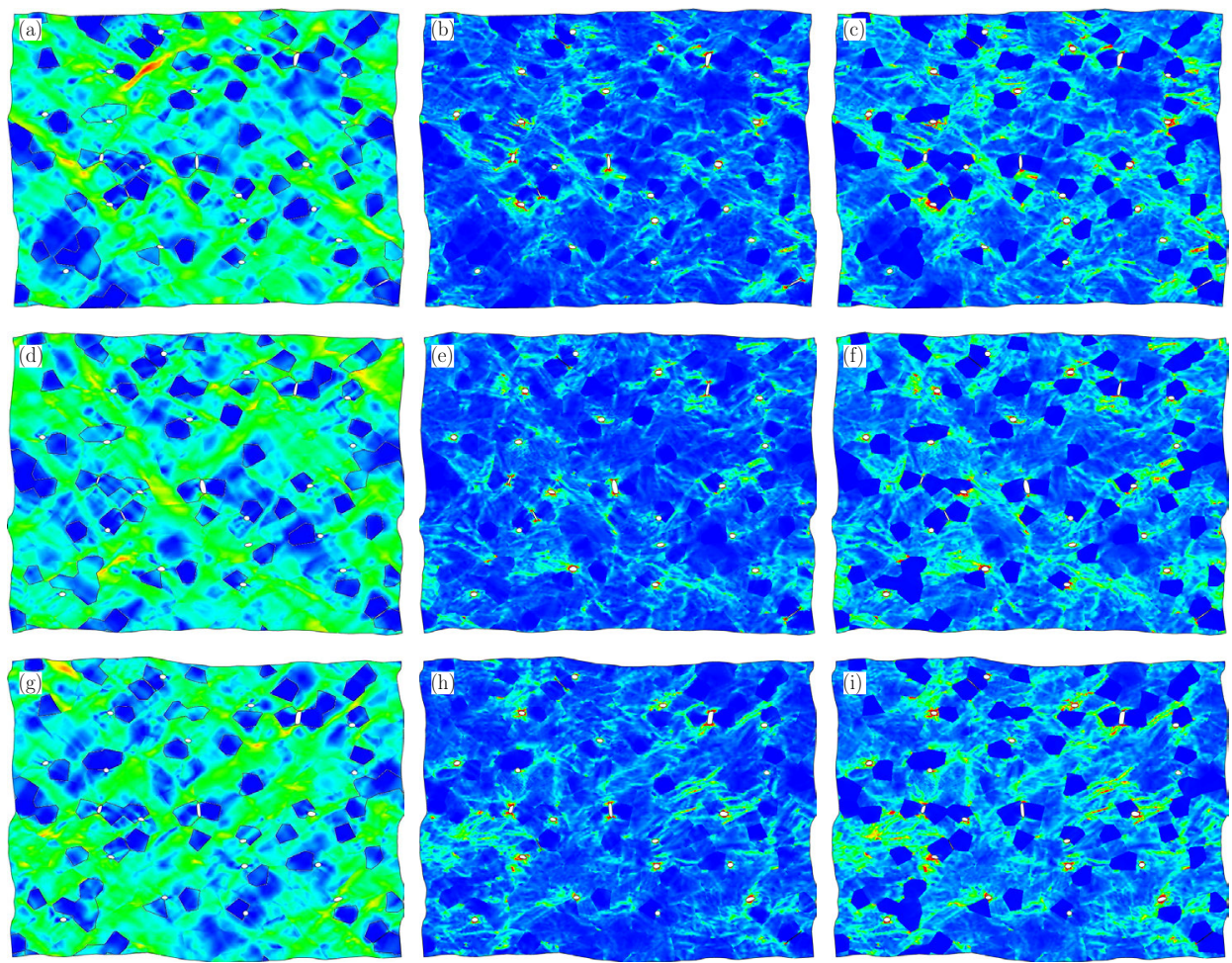

Figure 7.25: Distribution of (a,d and g) $\rho_{\mathrm{SSD}}\left(\mathrm{mm}^{-2}\right)$, (b,e and h) $\rho_{\mathrm{GND}}\left(\mathrm{mm}^{-2}\right),(\mathrm{c}, \mathrm{f}$ and i) Normalized dislocation density for random morphology. Three rows represent three different orientation sets. 


\subsubsection{Evolution of voids}

This section elaborates on the evolution of voids found in the RVEs in terms of total area of the voids as well as the individual area of each void. Figure 7.26 shows the increase in the total void area for two morphologies and three sets of orientations each. The voids are separated into two different classes namely the voids at the interface (solid lines) and the voids in ferrite grains (dashed lines).
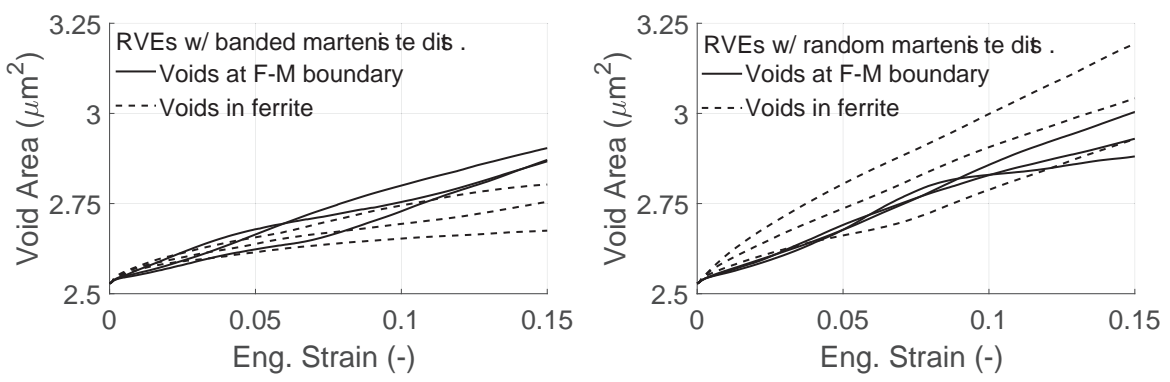

Figure 7.26: Change of total area of voids a) banded, b)random morphology for three orientation sets.

From Figure 7.26, we see the final average void area is bigger in the random morphology with an increase of $19.8 \%$, whereas it is $12.4 \%$ in the banded morphology. If we compare the different types of voids (in-grain \& at the interface), we see that the average void growth of in-grain voids was affected greatly by the martensite morphology, whereas interface voids seem to be less affected. At the end of deformation, the average area of all in-grain voids increases $20.7 \%$ from $2.53 \mu^{2}$ to $3.05 \mathrm{\mu m}^{2}$ in random morphology. For the banded morphology, the growth of the same type of voids is at $8.5 \%$ corresponding to a total area of $2.74 \mu^{2}$. However, there is a smaller difference between the average growth of interface voids for the two morphologies: $16.1 \%$ for random morphology and $13.8 \%$ for the banded morphology. We can relate the higher percentage of in-grain void growth to the higher deformation of ferrite in random morphology, which we can see from the higher SSD density value in ferrite and lower SSD density in martensite, Figure 7.23. The higher SSD density in ferrite in random structures suggests two things. First, it means that the ferrite is plastically deformed more for that structure. Secondly, it means that the ferrite has hardened more. Since deformation is prescribed on the RVE, the voids have to grow more. In other words, the mechanical contrast between the void and the ferrite increases as ferrite hardens, forcing the void to take a greater part in the deformation process. The storyline for interface voids is similar but this time the deformation of martensite is also crucial. It seems that since martensite does not deform as much as ferrite it stabilizes the void; the growth of interface voids depends not only on deformation of ferrite but also on the deformation of martensite.

Figure 7.27 and 7.28 show the change of normalized void area for individual voids for only one set of orientations per structure. The first observation on the curves of the figures is that all voids evolved differently from each other. The varying behavior of 
the voids can be attributed to (i) the orientation of the grains with respect to the loading axis, (ii) distribution of plastic strain within the RVE. Secondly, for both void groups, there was at least one void which did not grow in size but shrank during the deformation. Shrinkage of voids strongly suggests that there was either shear or compressive stress state surrounding those voids. Moreover, some of the voids show an initially growing trend which during the deformation process turns into shrinkage or vice versa, which can be attributed to change of local stress state. This is an important result since it shows the difference in evolution between the local stress state and the evolution of macroscopically applied stress state. Thirdly, in both structures, there was at least an in-grain void which grows $50 \%$, which is four to seven times more than the other voids.
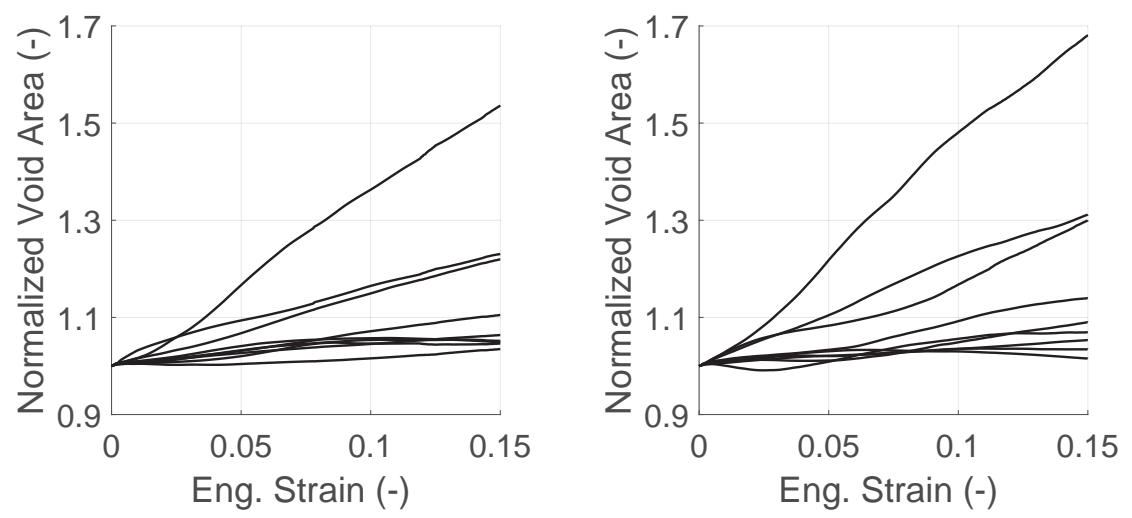

Figure 7.27: Normalized area of individual interface voids in banded (left) and random (right) morphology.
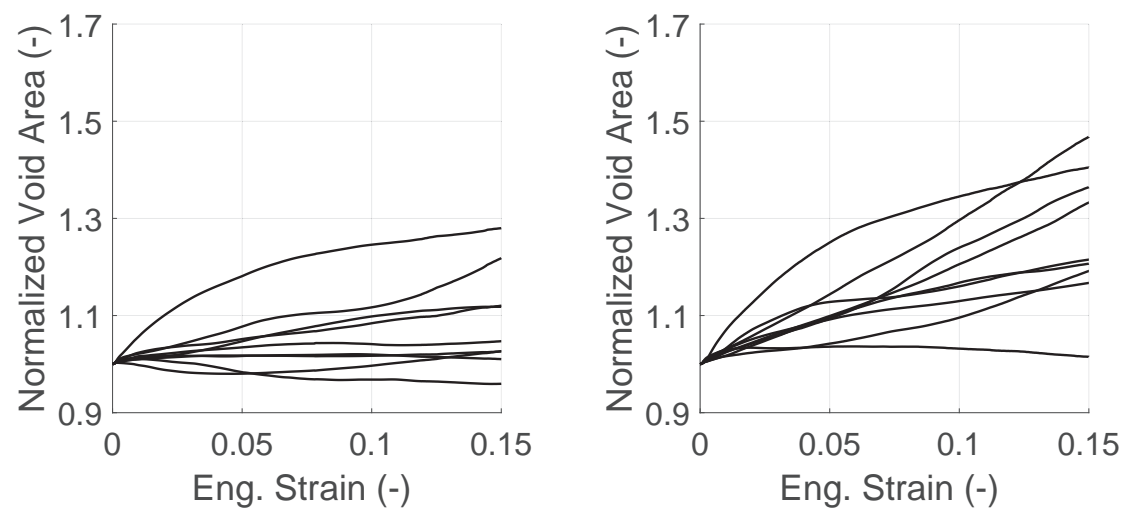

Figure 7.28: Normalized area of individual in-grain voids in banded (left) and random (right) morphology. 


\subsubsection{Evolution of voids formed by cracking of martensite}

In this part, we analyze the evolution of the total area of the voids formed by martensite cracks as well as the area of individual voids of that type. The area of these types of voids is defined as the area in the plane of the model, that is formed by opening of the faces of the martensite islands during deformation. In Figure 7.29, the damaged areas caused by the six voids were summed up, giving an overview on evolution of the voids in the structure. Figure ?? shows the evolution of the damaged area that is induced by individual cracks. Since the results between different orientation sets were similar this figure was plotted for only one orientation per morphology.

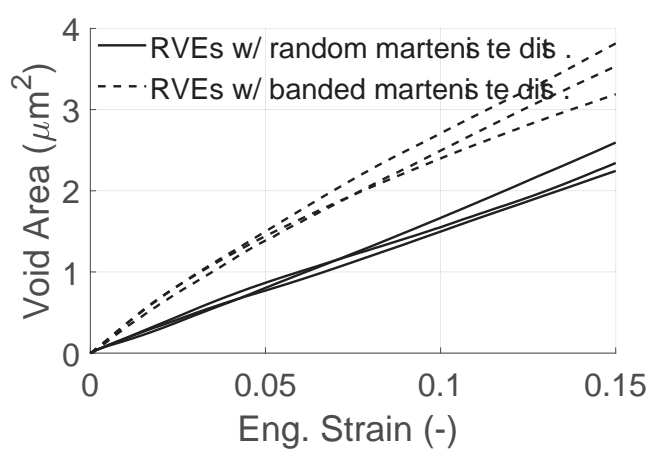

Figure 7.29: Change of total area of the voids formed by martensite cracks for three different orientation sets.

From Figure 7.29 it is seen that for all orientation sets of banded morphology, the growth of the crack area occurred faster than for random morphology. This behavior can be expected since the martensite islands in a banded structure act as strong fibers and they carry more load than their counterpart in a random structure. This explains the faster growth for banded morphology. This was also verified by the $\rho_{\mathrm{SSD}}$ and $\rho_{\text {GND }}$ distributions in Figure 7.23 and 7.24 where it can be seen that the average dislocation density of martensite in banded morphology is slightly higher than that of random morphology.

In Figure 7.30, it is seen that for both morphologies all cracks open, but at different rates. This can be explained by the variation in the local stress state surrounding the crack. Consequently, it is similar to the evolution of individual voids. The trends of different cracks in random morphology look more similar than in banded morphology. In the banded morphology, there is only one case that shows a saturating trend. The voids formed by martensite cracking grow in a more monotonic way - in the sense that growth trend does not change during the deformation - than the voids at the ferrite-martensite interfaces and in ferrite grains. 

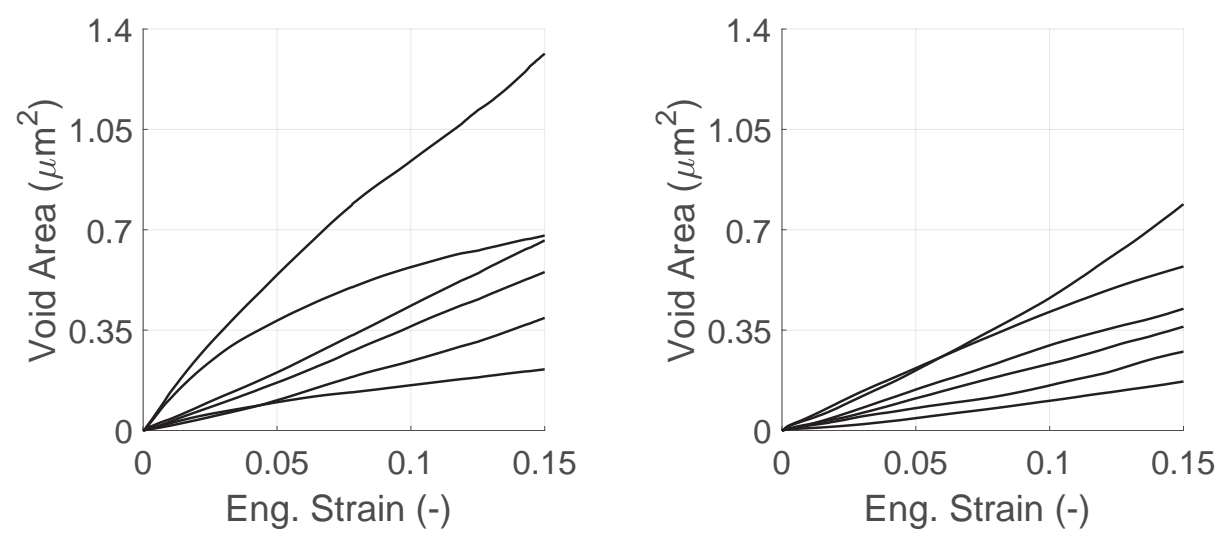

Figure 7.30: Area of individual voids formed by martensite cracking in banded (left) and random (right) morphology.

\subsection{Conclusions}

In this chapter, we have investigated the evolution of a void in a single phase polycrystal and evolution of voids in a more complicated dual phase microstructure. In the dual phase structure, we compared the effect of martensite morphology on damage evolution mechanisms in DP steels within a strain gradient enhanced crystal plasticity framework, which enables incorporation of plastic anisotropy as well as microstructural size effects. Two industrially relevant martensite morphologies (banded and random) are investigated by generated artificial 2D RVEs. To the RVEs, the most common damage mechanisms — voids formed by martensite cracks, voids between ferrite and martensite, and voids in ferrite grains - were introduced. The single phase one void investigations showed that;

- Void evolution is highly dependent on the lattice orientation of the grain.

- Void growth is effected from grain-grain interactions due to its influence on local deformation structure.

- Larger voids tend to grow bigger and interact with other grains.

After 15\% uniaxial deformation of the dual phase structure it was found that;

- When compared to random morphology, banded morphology results in more loading in martensite and less loading in ferrite.

- Random morphology causes the formation of finer shear bands.

- The local stress state surrounding damage occurrences determines their evolution characteristics. Therefore, an accurate prediction of the stress state is necessary. 
- The deformation in an RVE is highly heterogeneous causing a considerable numbers of GNDs. Hence, the model that is used in this scale should consider the strain gradient effects.

- In-grain voids grow more rapidly in random morphology, while interface voids grow faster in banded morphology.

- Evolution of individual voids shows that the local stress state deviates from the applied average stress state and the local stress state changes during deformation.

- Some voids reduced in size during deformation which shows they were under compressive or shear stress state. This was not observed in single phase simulations. This indicates the presence of mechanically diverse $2^{\text {nd }}$ phase results an increase in the deviation of local stress state.

- Voids caused by martensite cracks is a more severe mechanism since it has higher growth rates. 


\section{Conclusions and Recommendations}

The aim of this thesis is to investigate the effect of microstructural features on the initiation and evolution of damage by mechanisms in two phase metals, specifically, ferritic-martensitic dual phase steels.

The heterogeneity of plastic deformation, evolution of lattice orientations, active damage mechanisms and distribution and evolution of damage events are elaborated experimentally in Chapters 3 and 4 . The strain gradient enhanced rate-independent crystal plasticity framework, which is described in Chapter 5 was used in the subsequent numerical analysis. The effect of martensite content and distribution on stress and strain partitioning as well as damage initiation was investigated in Chapter 6. Evolution of damage mechanisms for different martensite distributions was studied in Chapter 7.

As a result of this work, a physical insight has been gained on the deformation characteristics of dual phase steels focusing on the influence of $2^{\text {nd }}$ phase characteristics on the evolution of plasticity and damage.

The main conclusions and important findings are summarized and some further research questions are given in the following sections.

\section{Experiments}

Electron backscatter diffraction (EBSD) analysis on the non-deformed DP600 steel sheet revealed the presence of small orientation variations and hence geometrically necessary dislocations (GND) in ferrite. The average GND density of ferrite was calculated as $2.6 \times 10^{8} \mathrm{~mm}^{-2}$ and the GNDs were concentrated very locally at ferrite grain boundaries. 
Tensile deformation (along rolling direction of the sheet) increased the spread in lattice orientation of the grains and therefore the GND content, which are caused by nonuniform plastic deformation. Spatial distribution of both in-grain orientation deviations were highly heterogeneous and therefore the distribution of the GNDs. The GND density was specifically higher than average around martensite islands. Moreover, increase in the GND density exhibited a saturating trend at around $4.0 \times$ $10^{9} \mathrm{~mm}^{-2}$ for ferrite and martensite.

EBSD analysis showed the presence of higher GND density than average at the vicinity of the nucleated voids. However, these locations were not necessarily the positions of the highest misorientation deviation or GND content in a grain. This suggested that void nucleation was not controlled solely by plastic strain.

Metallographic investigations on deformed DP600 steel sheet showed that there were two active damage mechanisms. In order of importance these mechanisms were void formation by cracking of martensite and void formation at the ferrite grain boundaries, mostly between ferrite and martensite.

The growth characteristics of the voids formed by these mechanisms were different from each other. Voids that are formed by cracking of martensite requires first failure of the martensite island almost perpendicular to the loading direction. Then the two sides of the failed martensite separate from each other by deformation of surrounding ferrite along the loading direction. As a result of this process, these voids had a thickness almost equal to the martensite island from which they from. In addition, due to the decrease in local load-bearing capability, these voids grow rapidly by plastic deformation of the surrounding ferrite. On the other hand, voids formed at the boundaries stay thin and grow mostly along the loading direction, causing a higher length to thickness ratio.

Interrupted tensile tests and metallographic investigations showed that void initiation started before the diffuse necking starts. Moreover, the number of voids and the size of the voids increased during the deformation process. A comparison between deformation along rolling and transverse direction showed that the rolling direction had a larger number of voids and damaged area.

Voids along the thickness direction of the sheet exhibited a nonuniform banded distribution. This distribution followed a similar trend to the distribution of martensite bands present at the sheet.

\section{Strain gradient enhanced crystal plasticity model}

A strain gradient enhanced rate-independent crystal plasticity model was implemented in order to capture the effect of microstructural features on plasticity such as lattice orientations, grain size and grain morphology. The gradient enhancement was realized by a lower order formulation based on slip rate gradients. The gradients were calculated explicitly in a staggered non-local approach after calculating slip rates by a Backward-Euler scheme for the integration points. The model was shown to be capable of predicting both structural and microstructural size 
effects. It was observed that the local stress and strain states are highly dependent on the local conditions and exhibit a nonuniform distribution. Moreover, the local stress and strain states varied from the macroscopically prescribed conditions on the RVE.

The model predicted a linear trend between the macroscopic yield strength of the RVE and the inverse square root of the grain size, $d^{-0.5}$, as suggested by the Hall-Petch relationship.

It was shown that the average statistically stored dislocation (SSD) density alters slightly due to grain-grain interactions. On the other hand, the average geometrically necessary dislocation (GND) density was directly affected from the decrease in grain size, resulting in additional hardening.

Decreasing grain size resulted in higher GND density mainly localized at the tripole junctions of the grains and grain boundaries. Further decrease in the grain size caused complex and highly heterogeneous GND distribution at the interior part of the grains.

\section{Damage in dual phase steels}

Numerical investigations revealed that the average stress and strain of a constituent phase (ferrite or martensite) in an RVE was affected by the $2^{\text {nd }}$ phase (martensite) content and distribution. It was found that an increase in the percentage of martensite increased the average stress and strain in both ferrite and martensite phases for a given strain level. Moreover, an increase in the martensite content did not influence the slope of the tie line joining stress and strain states of the phases which means the characteristics of stress and strain partitioning was not altered. On the other hand, a change in the distribution of martensite from random to banded alters the stress and strain partitioning towards a more isostrain (Voigt) like behavior when loaded along the banding direction.

When a comparison was made between locations of experimentally observed damage events and numerically found hot spots, it was seen that for both phases plastic work-based indicators gave reasonable resemblance. This suggests that a plastic work type indicator would be suitable in predicting susceptibility to damage of different microstructures.

The increase in martensite content resulted in an increase in the absolute (nonnormalized) values of damage indicators of both ferrite and martensite. This suggests that an increase in the percentage of martensite would trigger initiation of more damage events.

A change in the distribution of martensite from random to banded resulted in an increase in the possibility of damage in martensite while its effect on damage initiation in ferrite was negligible.

The effect of crystal orientation and void size on the evolution of pre-existing voids was investigated for single phase polycrystals. It was shown that the local stress and strain state surrounding the void and the orientation of the grain had an influence 
on the evolution characteristics. Moreover, voids with an initially smaller size grew slower than the voids with a larger size.

The effect of martensite distribution on the evolution of preexisting voids in a dual phase steel was investigated. Three types of voids were introduced in the RVEs namely voids formed by cracking of martensite, voids at the ferrite-martensite interface and voids inside ferrite grains. Evolution of the voids followed different trends, which was also the case for a single phase material. In addition, there were even some voids which got smaller during the deformation suggesting that they were under local shear or compression stress states even under the applied macroscopic tensile stretch. This also suggests that the variation in local stress states gets wider for more complicated microstructures. A monotonic trend in void evolution was not observed.

For both martensite distributions (random and banded), voids formed by martensite cracks grew faster than the pre-existing voids in ferrite grains and at the ferritemartensite interface. Moreover, the fastest growth occurred in the banded morphology for this type of voids.

The experimentally observed variation in the void distribution along the thickness and its correlation with martensite bands can be explained by the higher probability of damage initiation at martensite bands and the faster growth of the voids formed by cracking of martensite.

Voids at the ferrite-martensite boundary were not significantly affected by the martensite distribution since the evolution of these voids was constrained by the deformation of martensite. In addition, voids inside ferrite grains grew faster in random morphology than the banded morphology.

\section{Recommendations}

The phenomenological hardening function that is used should be changed into a physically-based hardening function which includes dislocation velocities. In this way the effects of strain rate and temperature can be captured by the model.

Theory on strain gradients does not include damage as a source of incompatibility. The theory should be further improved by understanding the effect of void nucleation on lattice rotations and the Burger tensor.

The secondary effects of $2^{\text {nd }}$ phase on the stress and strain partitioning should be added to the mean field models, which can be used in large scale simulations, in order to increase the prediction capability. This can be achieved by investigations conducted by using the implemented framework.

The conclusions on the effect of martensite content and distributions on damage initiation was not experimentally validated. An experimental study should be carried out with necessary heat-treatment steps and with the experimental approach in Chapter 4. 
A

\section{Slip systems}

Table A.1: Slip systems for FCC structure.

\begin{tabular}{lcc}
\hline Slip systems & Direction & Plane \\
\hline 1 & {$[\overline{1} 10]$} & $(111)$ \\
2 & {$[\overline{1} 01]$} & $(111)$ \\
3 & {$[0 \overline{1} 1]$} & $(111)$ \\
4 & {$[110]$} & $(\overline{1} 11)$ \\
5 & {$[101]$} & $(\overline{1} 11)$ \\
6 & {$[0 \overline{1} 1]$} & $(\overline{1} 11)$ \\
7 & {$[110]$} & $(1 \overline{1} 1)$ \\
8 & {$[\overline{1} 01]$} & $(1 \overline{1} 1)$ \\
9 & {$[011]$} & $(1 \overline{1} 1)$ \\
10 & {$[\overline{1} 10]$} & $(\overline{1} \overline{1} 1)$ \\
11 & {$[101]$} & $(\overline{1} \overline{1} 1)$ \\
12 & {$[011]$} & $(\overline{1} \overline{1} 1)$ \\
\hline
\end{tabular}


Table A.2: Slip systems for BCC structure.

\begin{tabular}{lcc}
\hline Slip systems & Direction & Plane \\
\hline 1 & {$[111]$} & $(1 \overline{1} 0)$ \\
2 & {$[11 \overline{1}]$} & $(1 \overline{1} 0)$ \\
3 & {$[1 \overline{1} 1]$} & $(110)$ \\
4 & {$[1 \overline{1} \overline{1}]$} & $(110)$ \\
5 & {$[111]$} & $(10 \overline{1})$ \\
6 & {$[11 \overline{1}]$} & $(101)$ \\
7 & {$[1 \overline{1} \overline{1}]$} & $(10 \overline{1})$ \\
8 & {$[1 \overline{1} \overline{1}]$} & $(101)$ \\
9 & {$[111]$} & $(01 \overline{1})$ \\
10 & {$[11 \overline{1}]$} & $(011)$ \\
11 & {$[1 \overline{1} 1]$} & $(011)$ \\
12 & {$[1 \overline{1} \overline{1}]$} & $(01 \overline{1})$ \\
13 & {$[111]$} & $(11 \overline{2})$ \\
14 & {$[11 \overline{1}]$} & $(112)$ \\
15 & {$[1 \overline{1} 1]$} & $(1 \overline{1} \overline{2})$ \\
16 & {$[1 \overline{1} \overline{1}]$} & $(1 \overline{1} 2)$ \\
17 & {$[111]$} & $(1 \overline{2} 1)$ \\
18 & {$[11 \overline{1}]$} & $(1 \overline{2} \overline{1})$ \\
19 & {$[1 \overline{1} 1]$} & $(121)$ \\
20 & {$[1 \overline{1} \overline{1}]$} & $(12 \overline{1})$ \\
21 & {$[111]$} & $(2 \overline{1} \overline{1})$ \\
22 & {$[11 \overline{1}]$} & $(2 \overline{1} 1)$ \\
23 & {$[1 \overline{1} 1]$} & $(21 \overline{1})$ \\
24 & {$[1 \overline{1} \overline{1}]$} & $(211)$ \\
\hline
\end{tabular}


Table A.3: Slip systems for BCC structure with non-Schmid effects.

\begin{tabular}{lccc}
\hline Slip systems & Direction & Plane & Plane $_{30^{\circ}}$ \\
\hline 1 & {$[111]$} & $(01 \overline{1})$ & $(\overline{1} 10)$ \\
2 & {$[111]$} & $(\overline{1} 01)$ & $(0 \overline{1} 1)$ \\
3 & {$[111]$} & $(1 \overline{1} 0)$ & $(10 \overline{1})$ \\
4 & {$[\overline{1} 11]$} & $(\overline{1} 0 \overline{1})$ & $(\overline{1} \overline{1} 0)$ \\
5 & {$[\overline{1} 11]$} & $(0 \overline{1} 1)$ & $(101)$ \\
6 & {$[\overline{1} 11]$} & $(110)$ & $(01 \overline{1})$ \\
7 & {$[\overline{1} \overline{1} 1]$} & $(0 \overline{1} \overline{1})$ & $(1 \overline{1} 0)$ \\
8 & {$[\overline{1} \overline{1} 1]$} & $(101)$ & $(011)$ \\
9 & {$[\overline{1} \overline{1} 1]$} & $(\overline{1} 10)$ & $(\overline{1} 0 \overline{1})$ \\
10 & {$[1 \overline{1} 1]$} & $(10 \overline{1})$ & $(110)$ \\
11 & {$[1 \overline{1} 1]$} & $(011)$ & $(\overline{1} 01)$ \\
12 & {$[1 \overline{1} 1$} & $(\overline{1} \overline{1} 0)$ & $(0 \overline{1} \overline{1})$ \\
13 & {$[\overline{1} \overline{1} \overline{1}]$} & $(01 \overline{1})$ & $(10 \overline{1})$ \\
14 & {$[\overline{1} \overline{1}]$} & $(\overline{1} 01)$ & $(\overline{1} 10)$ \\
15 & {$[\overline{1} \overline{1} \overline{1}]$} & $(1 \overline{1} 0)$ & $(0 \overline{1} 1)$ \\
16 & {$[1 \overline{1} \overline{1}]$} & $(\overline{1} 0 \overline{1})$ & $(01 \overline{1})$ \\
17 & {$[1 \overline{1} \overline{1}]$} & $(0 \overline{1} 1)$ & $(\overline{1} \overline{1} 0)$ \\
18 & {$[1 \overline{1} \overline{1}]$} & $(110)$ & $(101)$ \\
19 & {$[11 \overline{1}]$} & $(0 \overline{1} \overline{1})$ & $(\overline{1} 0 \overline{1})$ \\
20 & {$[11 \overline{1}]$} & $(101)$ & $(1 \overline{1} 0)$ \\
21 & {$[11 \overline{1}]$} & $(\overline{1} 10)$ & $(011)$ \\
22 & {$[\overline{1} \overline{1} \overline{1}]$} & $(10 \overline{1})$ & $(0 \overline{1} \overline{1})$ \\
23 & {$[\overline{1} \overline{1} \overline{1}]$} & $(011)$ & $(110)$ \\
24 & {$[\overline{1} \overline{1} \overline{1}]$} & $(\overline{1} \overline{1} 0)$ & $(\overline{1} 01)$ \\
\hline
\end{tabular}

Plane $_{30^{\circ}}$ makes $30^{\circ}$ with the plane. 



\section{B}

\section{UMAT}

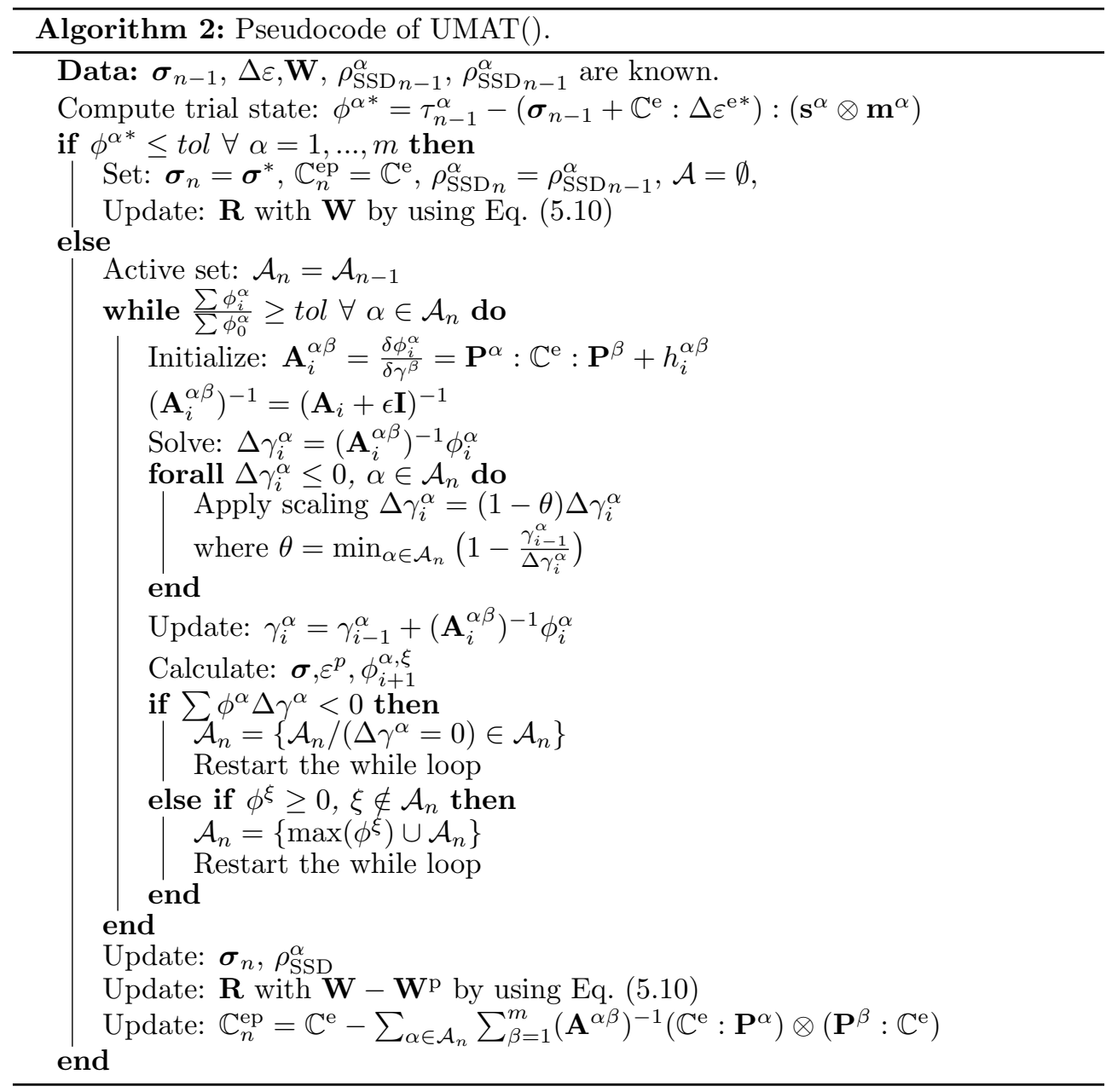





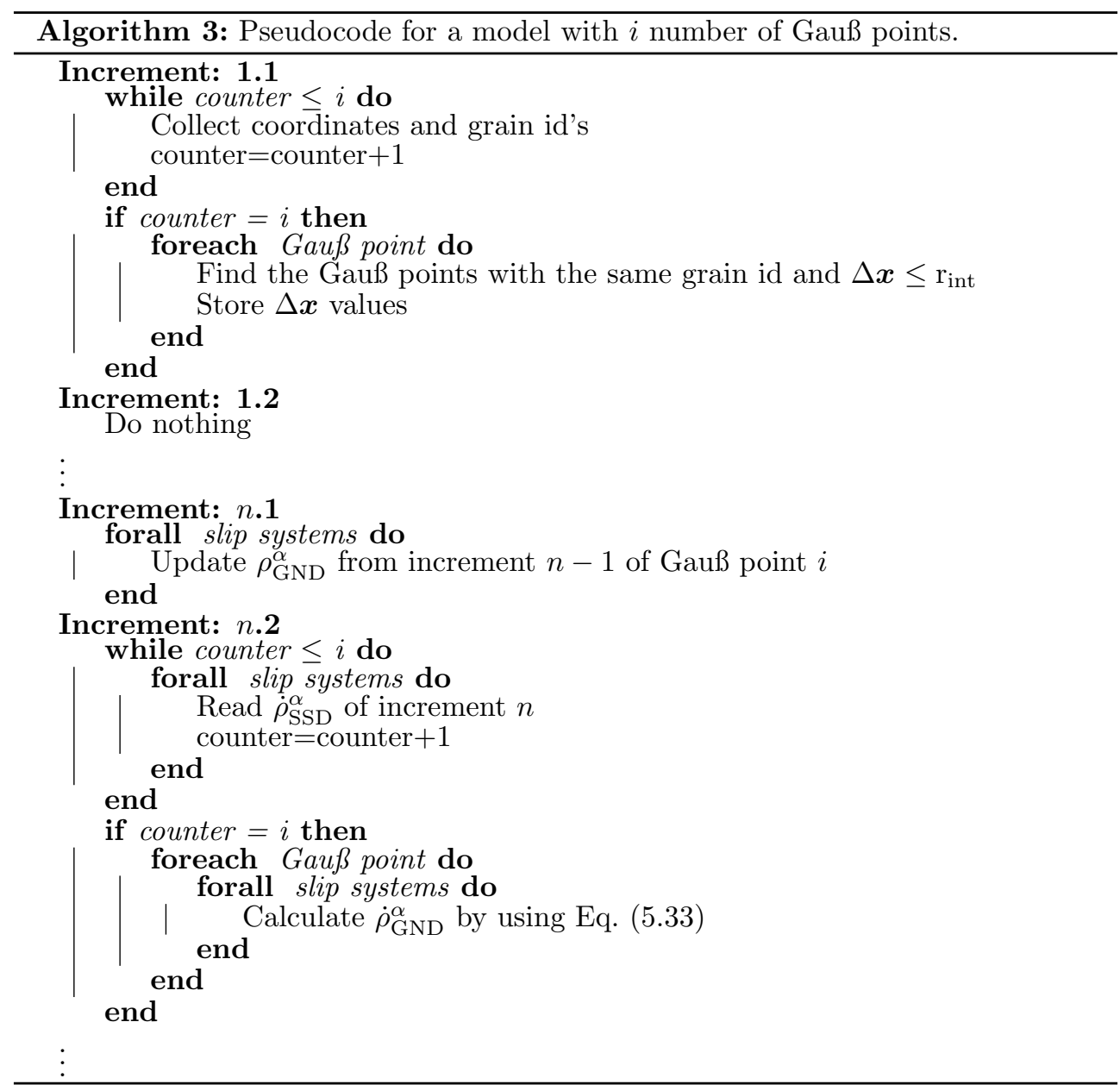

* At each increment $\operatorname{USDFLD}()$ is called before and after global equilibrium for all the Gauß points. 



\section{Nomenclature}

\section{Abbreviations}

$\begin{array}{ll}\text { AHSS } & \text { advanced high strength steel } \\ \text { BCC } & \text { body-centered cubic } \\ \text { DP } & \text { dual phase } \\ \text { DIC } & \text { digital image correlation } \\ \text { EDX } & \text { energy-dispersive X-ray spectroscopy } \\ \text { EBSD } & \text { electron backscatter diffraction } \\ \text { EBSP } & \text { electron backscatter pattern } \\ \text { FCC } & \text { face-centered cubic } \\ \text { GND } & \text { geometrically-necessary dislocation } \\ \text { GOS } & \text { grain orientation spread } \\ \text { GROD } & \text { grain reference orientation deviation } \\ \text { KAM } & \text { kernel average misorientation } \\ \text { ND } & \text { normal direction } \\ \text { RD } & \text { rolling direction } \\ \text { RVE } & \text { representative volume element } \\ \text { SEM } & \text { scanning electron microscope } \\ \text { SSD } & \text { statistically-stored dislocation } \\ \text { TD } & \text { transverse direction }\end{array}$




\section{Greek symbols}

$\begin{array}{ll}\alpha & \text { a slip system } \\ \dot{\gamma} & \text { slip rate } \\ \Delta & \text { difference; increment } \\ \varepsilon & \text { engineering strain } \\ \bar{\epsilon}^{\mathrm{p}} & \text { equivalent plastic strain } \\ \theta & \text { misorientation angle } \\ \mu & \text { shear modulus } \\ \nu & \text { Poisson's ratio } \\ \rho & \text { dislocation density } \\ \rho_{0} & \text { initial dislocation density } \\ \sigma & \text { Cauchy stress tensor } \\ \bar{\sigma} & \text { equivalent stress } \\ \sigma_{\mathrm{m}} & \text { hydrostatic stress } \\ \sigma_{j} & j \text { th principle Cauchy stress } \\ \tau & \text { resolved shear stress } \\ \tau_{0} & \text { lattice friction } \\ \tau_{\mathrm{f}} & \text { slip resistance } \\ \phi & \text { yield function }\end{array}$

\section{Roman symbols}

$\begin{array}{ll}b & \text { Burgers vector } \\ \mathbb{C}^{\mathrm{e}} & \text { elasticity tensor } \\ \mathrm{D} & \text { damage indicator } \\ \mathbf{D} & \text { rate of deformation tensor } \\ E & \text { elastic modulus } \\ \mathbf{F} & \text { deformation gradient } \\ \mathbf{F}_{\mathrm{e}} & \text { elastic part of deformation gradient } \\ \mathbf{F}_{\mathrm{i}} & \text { inelastic part of deformation gradient } \\ \mathbf{G} & \text { Burgers tensor, orientation matrix } \\ \mathbf{L} & \text { velocity gradient } \\ \mathbf{m} & \text { slip plane normal } \\ \mathbf{P}_{\mathrm{s}} & \text { Schmid tensor } \\ \mathbf{P}_{\mathrm{ns}} & \text { non-Schmid tensor } \\ \mathbf{Q} & \text { interaction matrix } \\ \mathbf{R} & \text { rotation tensor } \\ \mathbf{S} & \text { slip direction } \\ \mathbf{W} & \text { spin tensor }\end{array}$




\section{References}

[1] M.S. Rashid. Dual phase steels. Annual Review of Materials Research, 11:245266, 1981.

[2] W. Bleck. Cold-rolled, high-strength sheet steels for auto applications. JOM, 48(7):26-30, 1996.

[3] R. Kuziak, R. Kawalla, and S. Waengler. Advanced high strength steels for automotive industry. Archives of Civil and Mechanical Engineering, 8(2):103 $117,2008$.

[4] R.G. Davies. Influence of martensite composition and content on the properties of dual phase steels. Metallurgical Transactions A, 9(5):671-679, 1978.

[5] A.-P. Pierman, O. Bouaziz, T. Pardoen, P.J. Jacques, and L. Brassart. The influence of microstructure and composition on the plastic behaviour of dualphase steels. Acta Materialia, 73:298 - 311, 2014.

[6] H. Ghassemi-Armaki, R. Maaß, S.P. Bhat, S. Sriram, J.R. Greer, and K.S. Kumar. Deformation response of ferrite and martensite in a dual-phase steel. Acta Materialia, 62:197 - 211, 2014.

[7] C.C. Tasan, M. Diehl, D. Yan, M. Bechtold, F. Roters, L. Schemmann, C. Zheng, N. Peranio, D. Ponge, M. Koyama, K. Tsuzaki, and D. Raabe. An overview of dual-phase steels: Advances in microstructure-oriented processing and micromechanically guided design. Annual Review of Materials Research, 45(1):391-431, 2015.

[8] A.S. Argon, J. Im, and R. Safoglu. Cavity formation from inclusions in ductile fracture. Metallurgical Transactions A, 6(4):825-837, 1975.

[9] A.S. Argon and J. Im. Separation of second phase particles in spheroidized 1045 steel, cu-0.6pct cr alloy, and maraging steel in plastic straining. Metallurgical Transactions A, 6(4):839-851, 1975.

[10] A.A. Benzerga and J. Leblond. Ductile fracture by void growth to coalescence. Advances in Applied Mechanics, 44:169-305, 2010.

[11] F. Teirlinck, D.and Zok, J.D. Embury, and M.F. Ashby. Fracture mechanism maps in stress space. Acta Metallurgica, 36(5):1213-1228, 1988.

[12] B. Dodd and A.G. Atkins. Flow localization in shear deformation of voidcontaining and void-free solids. Acta Metallurgica, 31(1):9-15, 1983. 
[13] J. Crépin, T. Bretheau, and D. Caldemaison. Cavity growth and rupture of $\beta$-treated zirconium: a crystallographic model. Acta materialia, 44(12):49274935, 1996.

[14] T. Cox and J.R. Low. An investigation of the plastic fracture of aisi 4340 and 18 nickel-200 grade maraging steels. Metallurgical Transactions, 5(6):1457-1470, 1974.

[15] J.W. Hancock and A.C. Mackenzie. On the mechanisms of ductile failure in highstrength steels subjected to multi-axial stress-states. Journal of the Mechanics and Physics of Solids, 24(2):147 - 160, 1976.

[16] A. Weck, D.S. Wilkinson, E. Maire, and H. Toda. Visualization by x-ray tomography of void growth and coalescence leading to fracture in model materials. Acta Materialia, 56(12):2919 - 2928, 2008.

[17] F. Scheyvaerts, T. Pardoen, and P.R. Onck. A new model for void coalescence by internal necking. International Journal of Damage Mechanics, 19(1):95-126, 2010 .

[18] G. Avramovic-Cingara, Y. Ososkov, M.K. Jain, and D.S. Wilkinson. Effect of martensite distribution on damage behaviour in dp600 dual phase steels. Materials Science and Engineering: A, 516(1-2):7-16, 2009.

[19] J. Kadkhodapour, A. Butz, and S. Ziaei Rad. Mechanisms of void formation during tensile testing in a commercial, dual-phase steel. Acta Materialia, $59(7): 2575-2588,2011$.

[20] X. Sun, K.S. Choi, W.N. Liu, and M.A. Khaleel. Predicting failure modes and ductility of dual phase steels using plastic strain localization. International Journal of Plasticity, 25(10):1888-1909, 2009.

[21] J.P.M. Hoefnagels, C.C. Tasan, F. Maresca, F.J. Peters, and V.G. Kouznetsova. Retardation of plastic instability via damage-enabled microstrain delocalization. Journal of Materials Science, 50(21):6882-6897, 2015.

[22] K. Alharbi, H. Ghadbeigi, P. Efthymiadis, M. Zanganeh, S. Celotto, R. Dashwood, and C. Pinna. Damage in dual phase steel dp1000 investigated using digital image correlation and microstructure simulation. Modelling and Simulation in Materials Science and Engineering, 23(8):085005, 2015.

[23] E.E. Aşık, E.S. Perdahcıŏglu, and A.H. van den Boogaard. Microscopic investigation of damage mechanisms and anisotropic evolution of damage in dp600. Materials Science and Engineering: A, 739:348-356, 2019.

[24] G. Krauss. Solidification, segregation, and banding in carbon and alloy steels. Metallurgical and Materials Transactions B, 34(6):781-792, 2003.

[25] F. Archie, X.L. Li, and S. Zaefferer. Damage initiation in dual-phase steels: influence of crystallographic and morphological parameters. In Materials Science Forum, volume 879, pages 157-163. Trans Tech Publ, 2017.

[26] F. Archie, X. Li, and S. Zaefferer. Micro-damage initiation in ferrite-martensite dp microstructures: A statistical characterization of crystallographic and chemical parameters. Materials Science and Engineering: A, 701:302-313, 2017. 
[27] A.L. Gurson. Continuum theory of ductile rupture by void nucleation and growth: Part i-yield criteria and flow rules for porous ductile media. Journal of Engineering Materials and Technology, 99(1):2-15, 1977.

[28] V. Tvergaard and A. Needleman. Analysis of the cup-cone fracture in a round tensile bar. Acta metallurgica, 32(1):157-169, 1984.

[29] V. Tvergaard. Material failure by void growth to coalescence. Advances in applied Mechanics, 27:83-151, 1989.

[30] L.M. Kachanov. Time of the rupture process under creep conditions. Isv. Akad. Nauk. SSR. Otd Tekh. Nauk, 8:26-31, 1958.

[31] J. Lemaitre. Coupled elasto-plasticity and damage constitutive equations. Computer Methods in Applied Mechanics and Engineering, 51(1):31 - 49, 1985.

[32] L. Hao, P. Ke, and W. June. An anisotropic damage criterion for deformation instability and its application to forming limit analysis of metal plates. Engineering Fracture Mechanics, 21(5):1031-1054, 1985.

[33] J.L. Chaboche. Continuum damage mechanics: present state and future trends. Nuclear Engineering and Design, 105(1):19-33, 1987.

[34] J.L. Chaboche. Continuum damage mechanics: Part ii - damage growth, crack initiation, and crack growth. Journal of applied mechanics, 55(1):65-72, 1988.

[35] J. Lemaitre and R. Desmorat. Engineering damage mechanics: ductile, creep, fatigue and brittle failures. Springer Science \& Business Media, 2005.

[36] M. Jafari, S. Ziaei-Rad, N. Saeidi, and M. Jamshidian. Micromechanical analysis of martensite distribution on strain localization in dual phase steels by scanning electron microscopy and crystal plasticity simulation. Materials Science and Engineering: A, 670:57-67, 2016.

[37] J. Kadkhodapour, S. Butz, A.and Ziaei-Rad, and S. Schmauder. A micro mechanical study on failure initiation of dual phase steels under tension using single crystal plasticity model. International Journal of Plasticity, 27(7):1103$1125,2011$.

[38] T.W.J. De Geus, F. Maresca, R.H.J. Peerlings, and M.G.D. Geers. Microscopic plasticity and damage in two-phase steels: On the competing role of crystallography and phase contrast. Mechanics of Materials, 101:147-159, 2016.

[39] T.W.J. de Geus, R.H.J. Peerlings, and M.G.D. Geers. Competing damage mechanisms in a two-phase microstructure: How microstructure and loading conditions determine the onset of fracture. International Journal of Solids and Structures, 97:687-698, 2016.

[40] A. Ramazani, A. Schwedt, A. Aretz, U. Prahl, and W. Bleck. Characterization and modelling of failure initiation in dp steel. Computational materials science, 75:35-44, 2013.

[41] J.P. Bandstra and D.A. Koss. On the influence of void clusters on void growth and coalescence during ductile fracture. Acta Materialia, 56(16):4429-4439, 2008. 
[42] J.P. Bandstra, D.A. Koss, A. Geltmacher, P. Matic, and R.K. Everett. Modeling void coalescence during ductile fracture of a steel. Materials Science and Engineering: A, 366(2):269-281, 2004.

[43] T. Pardoen and J.W. Hutchinson. An extended model for void growth and coalescence. Journal of the Mechanics and Physics of Solids, 48(12):2467-2512, 2000 .

[44] U.F. Kocks. Polyslip in polycrystals. Acta Metallurgica, 6(2):85-94, 1958.

[45] K.S. Havner. Finite plastic deformation of crystalline solids. Cambridge University Press, 1992.

[46] E.O. Hall. The deformation and ageing of mild steel: Part iii discussion of results. Proceedings of the Physical Society. Section B, 64(9):747, 1951.

[47] A. Acharya and A.J. Beaudoin. Grain-size effect in viscoplastic polycrystals at moderate strains. Journal of the Mechanics and Physics of Solids, 48(10):22132230, 2000.

[48] J.F. Nye. Some geometrical relations in dislocated crystals. Acta metallurgica, $1(2): 153-162,1953$.

[49] M. Calcagnotto, D. Ponge, E. Demir, and D. Raabe. Orientation gradients and geometrically necessary dislocations in ultrafine grained dual-phase steels studied by 2d and 3d ebsd. Materials Science and Engineering: A, 527(1011):2738-2746, 2010.

[50] T. Schacht, N. Untermann, and E. Steck. The influence of crystallographic orientation on the deformation behaviour of single crystals containing microvoids. International Journal of Plasticity, 19(10):1605-1626, 2003.

[51] C. Schayes, J. Bouquerel, J. Vogt, F. Palleschi, and S. Zaefferer. A comparison of ebsd based strain indicators for the study of fe-3si steel subjected to cyclic loading. Materials Characterization, 115:61-70, 2016.

[52] F.J. Humphreys. Characterisation of fine-scale microstructures by electron backscatter diffraction (ebsd). Scripta materialigiia, 51(8):771-776, 2004.

[53] P.J. Hurley and F.J. Humphreys. The application of ebsd to the study of substructural development in a cold rolled single-phase aluminium alloy. Acta Materialia, 51(4):1087-1102, 2003.

[54] Z. Keshavarz and M.R. Barnett. Ebsd analysis of deformation modes in mg3al-1zn. Scripta Materialia, 55(10):915-918, 2006.

[55] R. Yoda, T. Yokomaku, and N. Tsuji. Plastic deformation and creep damage evaluations of type 316 austenitic stainless steels by ebsd. Materials characterization, 61(10):913-922, 2010.

[56] N. Allain-Bonasso, F. Wagner, S. Berbenni, and D.P. Field. A study of the heterogeneity of plastic deformation in if steel by ebsd. Materials Science and Engineering: A, 548:56-63, 2012.

[57] J. Bouquerel, M. Delbove, and J.-B. Vogt. Advanced processing of ebsd data to distinguish the complex microstructure evolution of a cu-ni-si alloy induced by fatigue. Materials Characterization, 145:556-562, 2018. 
[58] S.-J. Kim, S.-H. Kim, A.D. Rollett, K.H. Oh, H.N. Han, et al. Microtexture development during equibiaxial tensile deformation in monolithic and dual phase steels. Acta Materialia, 59(14):5462-5471, 2011.

[59] S.-S. Rui, Y.-B. Shang, Y.-N. Fan, Q.-N. Han, L.-S. Niu, H.-J. Shi, K. Hashimoto, and N. Komai. Ebsd analysis of creep deformation induced grain lattice distortion: A new method for creep damage evaluation of austenitic stainless steels. Materials Science and Engineering: A, 733:329-337, 2018.

[60] G.F. Vander Voort. Metallographic specimen preparation for electron backscattered diffraction. Part II. Pract Metallogr, 48(10):527-543, 2011.

[61] F. Bachmann, R. Hielscher, and H. Schaeben. Texture analysis with mtex-free and open source software toolbox. In Solid State Phenomena, volume 160, pages 63-68. Trans Tech Publ, 2010.

[62] J.-Y. Kang, H.K. Do, S.-I. Baik, T.-H. Ahn, Y.-W. Kim, Heung N. Han, K.H. Oh, H.-C. Lee, and S.H Han. Phase analysis of steels by grain-averaged ebsd functions. ISIJ international, 51(1):130-136, 2011.

[63] L. Ryde. Application of ebsd to analysis of microstructures in commercial steels. Materials Science and Technology, 22(11):1297-1306, 2006.

[64] L.P. Kubin and A. Mortensen. Geometrically necessary dislocations and straingradient plasticity: a few critical issues. Scripta materialia, 48(2):119-125, 2003.

[65] D. Yan, C.C. Tasan, and D. Raabe. High resolution in situ mapping of microstrain and microstructure evolution reveals damage resistance criteria in dual phase steels. Acta Materialia, 96:399-409, 2015.

[66] L. Schemmann, S. Zaefferer, D. Raabe, F. Friedel, and D. Mattissen. Alloying effects on microstructure formation of dual phase steels. Acta Materialia, 95:386-398, 2015.

[67] E. Demir, D. Raabe, N. Zaafarani, and S. Zaefferer. Investigation of the indentation size effect through the measurement of the geometrically necessary dislocations beneath small indents of different depths using ebsd tomography. Acta Materialia, 57(2):559-569, 2009.

[68] C.C. Tasan, J.P.M. Hoefnagels, M. Diehl, D. Yan, F. Roters, and D. Raabe. Strain localization and damage in dual phase steels investigated by coupled insitu deformation experiments and crystal plasticity simulations. International Journal of Plasticity, 63:198 - 210, 2014. Deformation Tensors in Material Modeling in Honor of Prof. Otto T. Bruhns.

[69] J. Kang, Y. Ososkov, J.D. Embury, and D.S. Wilkinson. Digital image correlation studies for microscopic strain distribution and damage in dual phase steels. Scripta Materialia, 56(11):999 - 1002, 2007.

[70] M. Mazinani and W.J. Poole. Effect of martensite plasticity on the deformation behavior of a low-carbon dual-phase steel. Metallurgical and Materials Transactions A, 38(2):328-339, 2007.

[71] Y.L. Su and J. Gurland. Strain partition, uniform elongation and fracture strain in dual-phase steels. Materials Science and Engineering, 95:151 - 165, 1987. 
[72] A. Fillafer, C. Krempaszky, and E. Werner. On strain partitioning and microdamage behavior of dual-phase steels. Materials Science and Engineering: A, 614:180 - 192, 2014.

[73] H. Toda, A. Takijiri, M. Azuma, S. Yabu, K. Hayashi, D. Seo, M. Kobayashi, K. Hirayama, A. Takeuchi, and K. Uesugi. Damage micromechanisms in dual-phase steel investigated with combined phase- and absorption-contrast tomography. Acta Materialia, 126:401 - 412, 2017.

[74] E. Maire, O. Bouaziz, M. Di Michiel, and C. Verdu. Initiation and growth of damage in a dual-phase steel observed by x-ray microtomography. Acta Materialia, 56(18):4954 - 4964, 2008.

[75] C.C. Tasan. Micro-mechanical characterization of ductile damage in sheet metal. $\mathrm{PhD}$ thesis, Technische Universiteit Eindhoven, 2010.

[76] D.L. Steinbrunner, D.K. Matlock, and G. Krauss. Void formation during tensile testing of dual phase steels. Metallurgical Transactions A, 19(3):579-589, 1988.

[77] M. Calcagnotto, Y. Adachi, D. Ponge, and D. Raabe. Deformation and fracture mechanisms in fine- and ultrafine-grained ferrite/martensite dual-phase steels and the effect of aging. Acta Materialia, 59(2):658-670, 2011.

[78] H. Ghadbeigi, C. Pinna, and S. Celotto. Failure mechanisms in dp600 steel: Initiation, evolution and fracture. Materials Science and Engineering: A, 588:420 - 431, 2013.

[79] Q. Lai, O. Bouaziz, M. Gouné, L. Brassart, M. Verdier, G. Parry, A. Perlade, Y. Bréchet, and T. Pardoen. Damage and fracture of dual-phase steels: Influence of martensite volume fraction. Materials Science and Engineering: A, 646:322 - 331, 2015.

[80] J.D. Verhoeven. A review of microsegregation induced banding phenomena in steels. Journal of Materials Engineering and Performance, 9(3):286-296, Jun 2000 .

[81] R.A. Grange. Effect of microstructural banding in steel. Metallurgical Transactions, 2(2):417-426, Feb 1971.

[82] ASTM Standard. E8," standard test methods for tension testing of metallic materials. Annual book of ASTM standards, 3:57-72, 2004.

[83] K. Park, M. Nishiyama, N. Nakada, T. Tsuchiyama, and S. Takaki. Effect of the martensite distribution on the strain hardening and ductile fracture behaviors in dual-phase steel. Materials Science and Engineering: A, 604:135 - 141, 2014.

[84] Russell A.K. Computer determination of the constituent structure of biological images. Computers and Biomedical Research, 4(3):315 - 328, 1971.

[85] H. Ghadbeigi, C. Pinna, S. Celotto, and J.R. Yates. Local plastic strain evolution in a high strength dual-phase steel. Materials Science and Engineering: A, 527(18):5026 - 5032, 2010.

[86] T.J. Baker, K.B. Gave, and J.A. Charles. Inclusion deformation and toughness anisotropy in hot-rolled steels. Metals Technology, 3(1):183-193, 1976. 
[87] S.-K. Han and H. Margolin. Void formation, void growth and tensile fracture of plain carbon steel and a dual-phase steel. Materials Science and Engineering: A, 112:133 - 141, 1989 .

[88] Q. Lai, O. Bouaziz, M. Gouné, L. Brassart, M. Verdier, G. Parry, A. Perlade, Y. Bréchet, and T. Pardoen. Damage and fracture of dual-phase steels: Influence of martensite volume fraction. Materials Science and Engineering: A, 646:322 - 331, 2015.

[89] M.F. Ashby. The deformation of plastically non-homogeneous materials. Philosophical Magazine, 21(170):399-424, 1970.

[90] A.H. Cottrell. Lx. the formation of immobile dislocations during slip. The London, Edinburgh, and Dublin Philosophical Magazine and Journal of Science, 43(341):645-647, 1952.

[91] R. Lagneborg and B.-H. Forsen. A model based on dislocation distributions for work-hardening and the density of mobile and immobile dislocations during plastic flow. Acta Metallurgica, 21(6):781-790, 1973.

[92] A. Acharya and J.L. Bassani. Lattice incompatibility and a gradient theory of crystal plasticity. Journal of the Mechanics and Physics of Solids, 48(8):15651595, 2000.

[93] N.A. Fleck, G.M. Muller, M.F. Ashby, and J.W. Hutchinson. Strain gradient plasticity: theory and experiment. Acta Metallurgica et materialia, 42(2):475487, 1994.

[94] N.A. Fleck and J.W. Hutchinson. A reformulation of strain gradient plasticity. Journal of the Mechanics and Physics of Solids, 49(10):2245-2271, 2001.

[95] P. Gudmundson. A unified treatment of strain gradient plasticity. Journal of the Mechanics and Physics of Solids, 52(6):1379-1406, 2004.

[96] C.F. Niordson and J.W. Hutchinson. On lower order strain gradient plasticity theories. European Journal of Mechanics-A/Solids, 22(6):771-778, 2003.

[97] E. Martínez-Pañeda, C.F. Niordson, and L. Bardella. A finite element framework for distortion gradient plasticity with applications to bending of thin foils. International Journal of Solids and Structures, 96:288-299, 2016.

[98] L.P. Evers, W.A.M. Brekelmans, and M.G.D. Geers. Non-local crystal plasticity model with intrinsic ssd and gnd effects. Journal of the Mechanics and Physics of Solids, 52(10):2379-2401, 2004.

[99] T. Yalcinkaya, W.A.M. Brekelmans, and M.G.D. Geers. Deformation patterning driven by rate dependent non-convex strain gradient plasticity. Journal of the Mechanics and Physics of Solids, 59(1):1-17, 2011.

[100] S. Bargmann and M. Ekh. Microscopic temperature field prediction during adiabatic loading using gradient extended crystal plasticity. International Journal of Solids and Structures, 50(6):899-906, 2013.

[101] B. Svendsen and S. Bargmann. On the continuum thermodynamic rate variational formulation of models for extended crystal plasticity at large deformation. Journal of the Mechanics and Physics of Solids, 58(9):1253-1271, 2010 . 
[102] M.E. Gurtin and L. Anand. A gradient theory for single-crystal plasticity. Modelling and Simulation in Materials Science and Engineering, 15(1):S263, 2006.

[103] M. Kuroda and V. Tvergaard. Studies of scale dependent crystal viscoplasticity models. Journal of the Mechanics and Physics of Solids, 54(9):1789-1810, 2006.

[104] L. Bardella. A deformation theory of strain gradient crystal plasticity that accounts for geometrically necessary dislocations. Journal of the Mechanics and Physics of Solids, 54(1):128-160, 2006.

[105] X.-L. Peng and G.-Y. Huang. Modeling dislocation absorption by surfaces within the framework of strain gradient crystal plasticity. International Journal of Solids and Structures, 72:98-107, 2015.

[106] N. Ohno and D. Okumura. Higher-order stress and grain size effects due to self-energy of geometrically necessary dislocations. Journal of the Mechanics and Physics of Solids, 55(9):1879-1898, 2007.

[107] G.Z. Voyiadjis and B. Deliktas. Mechanics of strain gradient plasticity with particular reference to decomposition of the state variables into energetic and dissipative components. International Journal of Engineering Science, 47(1112):1405-1423, 2009.

[108] M. Becker. Incompatibility and instability based size effects in crystals and composites at finite elastoplastic strains. University of Stuttgart, 2006.

[109] B.D. Reddy. The role of dissipation and defect energy in variational formulations of problems in strain-gradient plasticity. part 1: polycrystalline plasticity. Continuum Mechanics and Thermodynamics, 23(6):527-549, 2011.

[110] C. Nellemann, C.F. Niordson, and K.L. Nielsen. Hardening and strengthening behavior in rate-independent strain gradient crystal plasticity. European Journal of Mechanics-A/Solids, 67:157-168, 2018.

[111] N.A. Fleck, J.W. Hutchinson, and J.R. Willis. Strain gradient plasticity under non-proportional loading. Proceedings of the Royal Society A: Mathematical, Physical and Engineering Sciences, 470(2170):20140267, 2014.

[112] K.L. Nielsen and C.F. Niordson. A numerical basis for strain-gradient plasticity theory: rate-independent and rate-dependent formulations. Journal of the Mechanics and Physics of Solids, 63:113-127, 2014.

[113] C. Miehe and J. Schröder. A comparative study of stress update algorithms for rate-independent and rate-dependent crystal plasticity. International Journal for Numerical Methods in Engineering, 50(2):273-298, 2001.

[114] J. Mandel. Généralisation de la théorie de plasticité de W.T. koiter. International Journal of Solids and structures, 1(3):273-295, 1965.

[115] J.R. Rice. Inelastic constitutive relations for solids: an internal-variable theory and its application to metal plasticity. Journal of the Mechanics and Physics of Solids, 19(6):433-455, 1971.

[116] M.E. Gurtin and B.D. Reddy. Some issues associated with the intermediate space in single-crystal plasticity. Journal of the Mechanics and Physics of Solids, 95:230-238, 2016. 
[117] L. Anand and M. Kothari. A computational procedure for rate-independent crystal plasticity. Journal of the Mechanics and Physics of Solids, 44(4):525$558,1996$.

[118] T.J.R. Hughes and J. Winget. Finite rotation effects in numerical integration of rate constitutive equations arising in large-deformation analysis. International journal for numerical methods in engineering, 15(12):1862-1867, 1980.

[119] K.K. Mathur and P.R. Dawson. On modeling the development of crystallographic texture in bulk forming processes. International Journal of Plasticity, 5(1):67-94, 1989.

[120] CAE ABAQUS. Analysis user's manual, version 6.12, 2012.

[121] M. Zecevic, R.J. McCabe, and M. Knezevic. A new implementation of the spectral crystal plasticity framework in implicit finite elements. Mechanics of Materials, 84:114-126, 2015.

[122] R. Gröger, V. Racherla, J.L. Bassani, and V. Vitek. Multiscale modeling of plastic deformation of molybdenum and tungsten: Ii. yield criterion for single crystals based on atomistic studies of glide of $1 / 2<111>$ screw dislocations. Acta Materialia, 56(19):5412-5425, 2008.

[123] A. Patra, T. Zhu, and D.L. McDowell. Constitutive equations for modeling non-schmid effects in single crystal bcc-fe at low and ambient temperatures. International Journal of Plasticity, 59:1-14, 2014.

[124] G. Po, Y. Cui, D. Rivera, D. Cereceda, T.D. Swinburne, J. Marian, and N. Ghoniem. A phenomenological dislocation mobility law for bcc metals. Acta Materialia, 119:123-135, 2016.

[125] Y.J. Lee, G. Subhash, and G. Ravichandran. Constitutive modeling of textured body-centered-cubic (bcc) polycrystals. International Journal of Plasticity, 15(6):625-645, 1999.

[126] D. Cereceda, M. Diehl, F. Roters, D. Raabe, J.M. Perlado, and J. Marian. Unraveling the temperature dependence of the yield strength in singlecrystal tungsten using atomistically-informed crystal plasticity calculations. International Journal of Plasticity, 78:242-265, 2016.

[127] T. Yalcinkaya, W.A.M. Brekelmans, and M.G.D. Geers. Bcc single crystal plasticity modeling and its experimental identification. Modelling and Simulation in Materials Science and Engineering, 16(8):085007, 2008.

[128] M.S. Duesbery and V. Vitek. Plastic anisotropy in bcc transition metals. Acta Materialia, 46(5):1481-1492, 1998.

[129] R. Gröger, A.G. Bailey, and V. Vitek. Multiscale modeling of plastic deformation of molybdenum and tungsten: I. atomistic studies of the core structure and glide of $1 / 2<111>$ screw dislocations at $0 \mathrm{k}$. Acta Materialia, 56(19):5401-5411, 2008.

[130] A. Koester, A. Ma, and A. Hartmaier. Atomistically informed crystal plasticity model for body-centered cubic iron. Acta Materialia, 60(9):3894-3901, 2012. 
[131] A. Mapar, H. Ghassemi-Armaki, F. Pourboghrat, and K.S. Kumar. A differential-exponential hardening law for non-schmid crystal plasticity finite element modeling of ferrite single crystals. International Journal of Plasticity, 91:268-299, 2017.

[132] G. I. Taylor. The mechanism of plastic deformation of crystals. part i.--theoretical. Proc. R. Soc. Lond. A, 145(855):362-387, 1934.

[133] P. Franciosi. Glide mechanisms in bcc crystals: an investigation of the case of $\alpha$-iron through multislip and latent hardening tests. Acta Metallurgica, 31(9):1331-1342, 1983.

[134] R. Madec and L.P. Kubin. Dislocation interactions and symmetries in bcc crystals. In IUTAM Symposium on Mesoscopic Dynamics of Fracture Process and Materials Strength, pages 69-78. Springer, 2004.

[135] S. Queyreau, G. Monnet, and B. Devincre. Slip systems interactions in $\alpha$ iron determined by dislocation dynamics simulations. International Journal of Plasticity, 25(2):361-377, 2009.

[136] E. Perdahcıŏ̆lu, C. Soyarslan, E. Aşık, T. van den Boogaard, and S. Bargmann. A class of rate-independent lower-order gradient plasticity theories: Implementation and application to disc torsion problem. Materials, 11(8):1425, 2018.

[137] M.E. Gurtin. The burgers vector and the flow of screw and edge dislocations in finite-deformation single-crystal plasticity. Journal of the Mechanics and Physics of Solids, 54(9):1882-1898, 2006.

[138] P. Cermelli and M.E. Gurtin. On the characterization of geometrically necessary dislocations in finite plasticity. Journal of the Mechanics and Physics of Solids, 49(7):1539-1568, 2001.

[139] M.E. Gurtin. A finite-deformation, gradient theory of single-crystal plasticity with free energy dependent on the accumulation of geometrically necessary dislocations. International Journal of Plasticity, 26(8):1073-1096, 2010.

[140] C. Miehe, J. Schröder, and J. Schotte. Computational homogenization analysis in finite plasticity simulation of texture development in polycrystalline materials. Computer methods in applied mechanics and engineering, 171(3):387418, 1999.

[141] T. Liszka and J. Orkisz. The finite difference method at arbitrary irregular grids and its application in applied mechanics. Computers and structures, 11(12):83-95, 1980 .

[142] E.S. Perdahcioglu, C. Soyarslan, A.H. van den Boogaard, and S. Bargmann. Gradient enhanced physically based plasticity: Implementation and application to a problem pertaining size effect: Implementation and application to a problem pertaining size effect. In ESAFORM 2016: 19th International ESAFORM Conference on Material Forming. American Institute of Physics, 2016.

[143] J. Mediavilla, R.H.J. Peerlings, and M.G.D. Geers. An integrated continuousdiscontinuous approach towards damage engineering in sheet metal forming processes. Engineering Fracture Mechanics, 73(7):895-916, 2006. 
[144] J. Fish, T. Jiang, and Z. Yuan. A staggered nonlocal multiscale model for a heterogeneous medium. International Journal for Numerical Methods in Engineering, 91(2):142-157, 2012.

[145] N. Hansen. The effect of grain size and strain on the tensile flow stress of aluminium at room temperature. Acta Metallurgica, 25(8):863-869, 1977.

[146] P. Van Liempt. Workhardening and substructural geometry of metals. Journal of Materials Processing Technology, 45(1-4):459-464, 1994.

[147] N.J. Petch. The cleavage strength of polycrystals. Journal of the Iron and Steel Institute, 174:25-28, 1953.

[148] J.K. Mackenzie. Second paper on statistics associated with the random disorientation of cubes. Biometrika, 45(1-2):229-240, 1958.

[149] B. Nijhuis. Investigation of the scope and limitations of gradient enhanced crystal plasticity in explaining macroscopic phenomena (msc thesis ). University of Twente, 2018.

[150] G.F. Vander Voort. Grain size measurement. In Practical applications of quantitative metallography. ASTM International, 1984.

[151] U. Borg. A strain gradient crystal plasticity analysis of grain size effects in polycrystals. European Journal of Mechanics-A/Solids, 26(2):313-324, 2007.

[152] M.G. Moghaddam, A. Achuthan, B.A. Bednarcyk, Steven M. Arnold, and E.J. Pineda. Grain size-dependent crystal plasticity constitutive model for polycrystal materials. Materials Science and Engineering: A, 703:521-532, 2017.

[153] K.S. Cheong, E.P. Busso, and A. Arsenlis. A study of microstructural length scale effects on the behaviour of fcc polycrystals using strain gradient concepts. International Journal of Plasticity, 21(9):1797-1814, 2005.

[154] M.A. Haque and M.T.A. Saif. Strain gradient effect in nanoscale thin films. Acta Materialia, 51(11):3053-3061, 2003.

[155] Z.N. Farhat, Y. Ding, D.O. Northwood, and A.T. Alpas. Effect of grain size on friction and wear of nanocrystalline aluminum. Materials Science and Engineering: A, 206(2):302-313, 1996.

[156] H.-H. Fu, D.J. Benson, and M.A. Meyers. Analytical and computational description of effect of grain size on yield stress of metals. Acta materialia, 49(13):2567-2582, 2001.

[157] K.S. Choi, W.N. Liu, X. Sun, and M.A. Khaleel. Influence of martensite mechanical properties on failure mode and ductility of dual-phase steels. Metallurgical and Materials Transactions A, 40(4):796-809, 2009.

[158] H. Kumar, C.L. Briant, and W.A. Curtin. Using microstructure reconstruction to model mechanical behavior in complex microstructures. Mechanics of Materials, 38(8-10):818-832, 2006.

[159] V. Uthaisangsuk, U. Prahl, and W. Bleck. Micromechanical modelling of damage behaviour of multiphase steels. Computational Materials Science, 43(1):27-35, 2008. 
[160] U. Prahl, S. Papaefthymiou, V. Uthaisangsuk, W. Bleck, J. Sietsma, and S. Van der Zwaag. Micromechanics-based modelling of properties and failure of multiphase steels. Computational Materials Science, 39(1):17-22, 2007.

[161] G.L. Povirk. Incorporation of microstructural information into models of twophase materials. Acta metallurgica et materialia, 43(8):3199-3206, 1995.

[162] T.W.J. de Geus, R.H.J. Peerlings, and M.G.D. Geers. Fracture in multi-phase materials: Why some microstructures are more critical than others. Engineering Fracture Mechanics, 169:354-370, 2017.

[163] T.W.J. de Geus. From damage to fracture, from micro to macro: a systematic study of ductile fracture in multi-phase microstructures. Technische Universiteit Eindhoven, 2016.

[164] M. Charleux, W.J. Poole, M. Militzer, and A. Deschamps. Precipitation behavior and its effect on strengthening of an hsla-nb/ti steel. Metallurgical and Materials Transactions A, 32(7):1635-1647, 2001.

[165] S. Morito, J. Nishikawa, and T. Maki. Dislocation density within lath martensite in fe-c and fe-ni alloys. ISIJ international, 43(9):1475-1477, 2003.

[166] C.C. Tasan, J.P.M. Hoefnagels, and M.G.D. Geers. Microstructural banding effects clarified through micrographic digital image correlation. Scripta Materialia, 62(11):835-838, 2010.

[167] Jacques Besson. Continuum models of ductile fracture: a review. International Journal of Damage Mechanics, 19:3 - 52, 2010.

[168] W.M. Garrison and N.R. Moody. Ductile fracture. Journal of Physics and Chemistry of Solids, 48(11):1035 - 1074, 1987.

[169] R.H. Stone, T.B. Cox, J.R. Low, and J.A. Psioda. Microstructural aspects of fracture by dimpled rupture. International Metals Reviews, 30(1):157-180, 1985.

[170] F.A. McClintock. A criterion for ductile fracture by the growth of holes. Journal of applied mechanics, 35(2):363-371, 1968.

[171] J. R. Rice and D. M. Tracey. On the ductile enlargement of voids in triaxial stress fields. Journal of the Mechanics and Physics of Solids, 17(3):201-217, 1969.

[172] J. Koplik and A. Needleman. Void growth and coalescence in porous plastic solids. International Journal of Solids and Structures, 24(8):835-853, 1988.

[173] V. Monchiet, O. Cazacu, E. Charkaluk, and D. Kondo. Macroscopic yield criteria for plastic anisotropic materials containing spheroidal voids. International Journal of Plasticity, 24(7):1158-1189, 2008.

[174] T.L. O'regan, D.F. Quinn, M.A. Howe, and P.E. McHugh. Void growth simulations in single crystals. Computational mechanics, 20(1-2):115-121, 1997.

[175] X. Han, J. Besson, S. Forest, B. Tanguy, and S. Bugat. A yield function for single crystals containing voids. International Journal of Solids and Structures, 50(14-15):2115-2131, 2013. 
[176] S.K. Yerra, C. Tekog, F. Scheyvaerts, L. Delannay, P. Van Houtte, T. Pardoen, et al. Void growth and coalescence in single crystals. International Journal of Solids and Structures, 47(7-8):1016-1029, 2010.

[177] R.A. Lebensohn, J.P. Escobedo, E.K. Cerreta, D. Dennis-Koller, C.A. Bronkhorst, and J.F. Bingert. Modeling void growth in polycrystalline materials. Acta Materialia, 61(18):6918-6932, 2013.

[178] J.Y. Shu. Scale-dependent deformation of porous single crystals. International Journal of Plasticity, 14(10-11):1085-1107, 1998.

[179] C. Ling, J. Besson, S. Forest, B. Tanguy, F. Latourte, and E. Bosso. An elastoviscoplastic model for porous single crystals at finite strains and its assessment based on unit cell simulations. International Journal of Plasticity, 84:58-87, 2016.

[180] U. Borg, C.F. Niordson, and J.W. Kysar. Size effects on void growth in single crystals with distributed voids. International Journal of Plasticity, 24(4):688$701,2008$.

[181] V. Tvergaard and C. Niordson. Nonlocal plasticity effects on interaction of different size voids. International Journal of Plasticity, 20(1):107-120, 2004.

[182] J. Segurado and J. LLorca. Discrete dislocation dynamics analysis of the effect of lattice orientation on void growth in single crystals. International Journal of Plasticity, 26(6):806-819, 2010.

[183] T. Tang, S. Kim, and M.F. Horstemeyer. Molecular dynamics simulations of void growth and coalescence in single crystal magnesium. Acta Materialia, 58(14):4742-4759, 2010.

[184] G.P. Potirniche, M.F. Horstemeyer, G.J. Wagner, and P.M. Gullett. A molecular dynamics study of void growth and coalescence in single crystal nickel. International Journal of Plasticity, 22(2):257-278, 2006.

[185] A. Ramazani, K. Mukherjee, U. Prahl, and W. Bleck. Modelling the effect of microstructural banding on the flow curve behaviour of dual-phase (dp) steels. Computational Materials Science, 52(1):46-54, 2012.

[186] S.K. Paul. Real microstructure based micromechanical model to simulate microstructural level deformation behavior and failure initiation in dp 590 steel. Materials \& Design, 44:397-406, 2013.

[187] P.J.J. Kok, W. Spanjer, and H. Vegter. A microstructure based model for the mechanical behavior of multiphase steels. In Key Engineering Materials, volume 651, pages 975-980. Trans Tech Publ, 2015.

[188] S. Yadegari, S. Turteltaub, A.S.J. Suiker, and P.J.J. Kok. Analysis of banded microstructures in multiphase steels assisted by transformation-induced plasticity. Computational Materials Science, 84:339-349, 2014. 



\section{Research deliverables}

The key deliverables of this research are listed in this section.

\section{Journal articles}

- E.E. Asik, E.S. Perdahcioglu, A.H. van den Boogaard. Microscopic investigation of damage mechanisms and anisotropic evolution of damage in DP600. Materials Science and Engineering: A, 739, 348-356, 2019.

- E. Perdahcioglu, C. Soyarslan, E. Asik, A.H van den Boogaard, S. Bargmann. A class of rate-independent lower-order gradient plasticity theories: Implementation and application to disc torsion problem. Materials, 11 (8), $1425,2018$.

- E.E. Asik, E.S. Perdahcioglu, A.H. van den Boogaard. An RVE based study of the effect of martensite banding on damage evolution in dual phase steels. Computational Materials Science (under review).

- E.E. Asik, E.S. Perdahcioglu, A.H. van den Boogaard. Evolution of lattice orientations and geometrically necessary dislocation density in dual phase steels under tensile deformation (submitted to Materials Science and Engineering: A).

- E.E. Asik, E.S. Perdahcioglu, A.H. van den Boogaard. A micro-mechanical study of damage initiation in dual phase steels (In preparation). 


\section{Conference proceedings}

- E.S. Perdahcioglu, E.E. Asik, A.H. van den Boogaard. Prediction of void growth using gradient enhanced polycrystal plasticity. American Institute of Physics Conference Series, 2113 (18), 2019.

- E.E. Asik, E.S. Perdahcioglu, A.H. van den Boogaard. Investigation of microstructural features on damage anisotropy. 11th Forming Technology Forum, 71-71, 2018.

- C. Soyarslan, E.S. Perdahcıoğlu, E.E. Asik, A.H. Van Den Boogaard, S. Bargmann. Implementation and application of a gradient enhanced crystal plasticity model. AIP Conference Proceedings, 1896, (160008), 2017.

\section{Presentations in conferences}

- International symposium, Nano and Micro Scale Damage in Metals, the Netherlands, 2018.

- M2i conference, Meeting Materials, the Netherlands, 2018.

- International Conference on Computational Plasticity-Fundamentals and Applications, Spain, 2017.

- Engineering Mechanics Symposium, the Netherlands, 2017.

\section{Poster contributions in conferences}

- M2i conference, Meeting Materials, the Netherlands, 2015, 2016, 2017, 2018.

- Engineering Mechanics Symposium, the Netherlands, 2015, 2016, 2017, 2018. 


\section{Acknowledgments}

This journey started a little more than 4 years ago in Utrecht while drinking beer and chatting with friends. Since that time a lot of personal and professional changes occurred in my life. As the $3^{\text {rd }}$ Doctor said: "A straight line may be the shortest distance between two points, but it is by no means the most interesting." Now, when the thesis is almost finished, it is a chance for me to thank all those people who contributed to my work and helped me get here.

Above all, I would like to express my sincere gratitude to Ton van den Boogaard, the chair of nonlinear solid mechanics and promoter of my thesis. Ton, thank you for giving me the chance to work on this project by accepting me in the group. I greatly appreciate the inspiring comments, endless support that you provided throughout my PhD. I would like to express my deepest appreciation to Semih Perdahcıoğlu, my daily supervisor and co-promoter of this thesis. Semih, thank you for never-ending encouragement and supporting me from the first day to the last. Many thanks for all the stimulating talks during these years. You have always been actively involved in my research, and I am very thankful for your patience, support, motivation, creativity and numerous advice on both scientific and non-scientific matters.

I would like to thank prof. Ton van den Boogaard, dr. Semih Perdahcıoğlu and Sharhzad Mirhosseini for carefully reading and providing valuable feedback on my thesis. I am also grateful to the committee members, dr. Ton Bor, prof. Seán Leen, prof. Jilt Sietsma and prof. Kees Venner for taking the time to evaluate my thesis. I am thankful for their valuable comments and their attendance to my defense. Additionally, the efforts of Lawrence Lowenthal for improving the language are highly appreciated.

This research was carried out in the framework of the Partnership Program of the Materials innovation institute M2i and the Foundation of Fundamental Research on Matter (FOM), which is part of the Netherlands Organization for Scientific Research (NWO). I would like to thank the people in M2i and FOM for arranging courses, meetings and conferences. I am also grateful to Jilt Sietsma for his leadership in Physics of Failure consortium. I would like to acknowledge Tata Steel R\&D, Ijmuiden for providing the material of this study. Many thanks to Eisso Atzema and Piet Kok for their insightful comments and inspiring discussions during this period. 
Special thanks to Debbie who helped with countless amount of paperwork and administrative stuff. Debbie, I am very grateful that you were there when we needed you. Many thanks also to Maria who took care of all the administrative issues on the FOM side. Additionally, I would like to thank Belinda for her help when Debbie was not present.

I would like to thank to Celal Soyarslan for his contributions and collaboration for the strain gradient enhancement methodology used in this work.

I am grateful to all my colleagues in the NSM group. Thank you very much for all the feedbacks on my project, enjoyable coffee breaks and group activities. I am especially grateful to Geijs and Javad for their useful comments and inputs during the Dieka meetings or afterwards. I would like to thank Ali for the fun times in the mechanical lab. Now, I have just remembered "Modulus Project". I would like to thank Nico for making sure the workstations are running and to Nick for keeping the SEM operational. A special thanks goes to my office mates: Annemieke, Daan, Farshad, Neda and Núbia. Thank you for creating a positive working environment.

Life in Enschede would not be same without some people and the linden trees. Ali and Mieke, thank you for organizing those lovely dinners and gatherings. Thank you for opening your house to us. Spa in Spa and pide were great. Special thanks for translating the summary to Dutch. Sikander, thank you for hosting all the biryani nigths and letting me move into your place even before you. Omid and Liwia, thanks for opening your balcony for the BBQs and the board game sessions. Melissa and Steve, thank you for introducing Killer Bunnies, the pit and s'mores. Jos, Frans, Iris, Farshad, Farnaz, Antonella, Laura, Björn, Jurnan, Boukje, Shyam, Nachiket, Shakil, Shahrzad, Mehdi... thank you for the cheerful moments.

I also would like to thank to Tankstation Crew for making Enschede a great place. A special thanks goes to Yasaman for bringing this lovely community to life and making us feel at home.

I would like to thank Ozan, Senem, Ozan Ç., Sinem, Didem, Pelin, Yiğitcan, Aysun, Yusuf, Derya, Devrim and Gül for their friendship. A very special thanks goes to my paranymphs Ozan and Senem for their assistance.

Thanks to Rasim, Özlem, Aliş, Neşe, Burak, Çado, Aybüke, Yunus, Dilay, Ako and İrem, who are scattered across the globe, for their long-distance friendship. Deniz, İpek, Aslı, Güven, Gülşah and Yüc: Thank you for traveling all the way to Enschede.

My parents, Meral and Tamer, deserve the greatest thanks for their infinite love and support. I would also like to thank Remziye and Necati, Aslı and Nizam, Pinar and Burhan, and Damla, Çınar and Toprak for bringing joy to my life. My final appreciations go to Slla. Thank you for being in my life as my best friend and my wife. Thank you for your endless support and patience. Thank you for always finding a way to cheer me up.

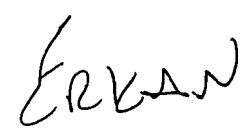

